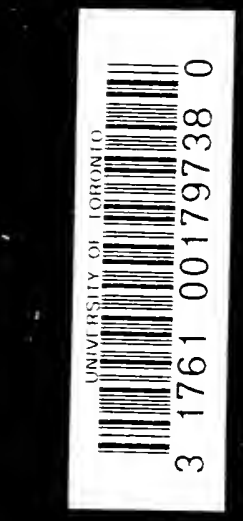









\title{
MATHEMATISCH.PHYSIKALISCHE SCHRIFTEN
}

\section{FÜR INGENIEURE UND STUDIERENDE}

HERAUSGEGEBEN VON E. JAHNKE

\section{MATHEMATISCHE INSTRUMENTE}

\author{
VON \\ PRof. DR. A. GALLE
}

ABTEILUNGSVORSTEHER AM KGL. GEODATISCHEN IMSTITUT $\mathrm{ZU}$ POTSDAM

MIT 86 ABBILDUNGEN UND FIGUREN

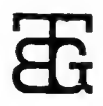

LEIPZIG UND BERLIN

DRUCK UND VERLAG VON B. G. TEUBNER 
QA

$$
7 i
$$

COPYRIGHT 1912 BY B. G.TEUBNER IN I.EIPZIG

ALLE RECHTE,
EINSCHLIESSLICH DES UUBERSETZUNGSRECHTS, VORBEHALTEN 


\section{Vorwort.}

Unter den Errungenschaften der Technik nehmen die ruathematischen Instrumente insofern eine besondere Stellung ein, als sie der Geistestätigkeit des Jlenschen einen Teil der Arbeit abnehmen. Nanche von ihnen, z. B. die Planimeter, sind für bestimmte Aufgaben unersetzbar geworden, andere, wie die dnalysatoren, sind geeignet, gewisse Zusammenbänge klarer hervortreten zu lassen, als sie die Rechnung ohne weiteres zeigt. Der Nutzen der meisten Apparate gibt sich bei büufiger Wiederholung derselben Operation in einer beträchtlichen Zeitersparnis zu erkennen. Auch wenn die erforderliche Genauigkeit in einigen Fiillen nicht durch das Instrument erreicht wird, so dient die dadurch erlangte Prüfung zur Sicherung des Ergebnisses der Rechnung.

Dem Scharfsinn, mit dem diese Hilfsmittel erdacht sinı, und der Feinheit und Genanigkeit ihrer Herstellung wird man die Bewunderung nicht versagen kïnnen. Hieraus entspringt der Wunsch, die Grundlagen und Einzelheiten ihrer Konstruktion kennen zu lernen. Die Kenntnis der Zusammensetzung der Apparate wird aber dann notwendig, wenn es sich um die Prüfung ihrer Genauigkeit und Zuverlässigkeit handelt. Für einzelne Aufgaben kann eine solche Cntersuchung eine Arbeitivermehrung bedeuten, bei lä̈ufiger Anwendung des Instrumentes fallt sie aber kaum ins Gewicht.

Beschreibungen und Erklärungen sind für viele Instrumente aus Anla $B$ ron Ausstellungen und in Auftrage von P'räzisionswerkstätten in Katalogen veröffentlicht worden. Einige Enzyklopädien geben, nehen Literaturnachweisen, meist in knapper Form eine Übersicht über die vorhandenen Konstruktionen zur raschen Orientierung des lachmannes. Es fehlt jedoch eine rusammenhängende Darstellung der Einrichtungen dieser Instrumente mit Erklärungen und Hinweisen auf ibre Anwendnng, die als Einführung in diesen Zweig der Technik dienen könnte. Diese Lücke beabsichtigt das vorliegende Bändchen auszufüllen, das in denjenigen Abschnitten, die es erlauben, so allgemein verständlich wie möglich gehalten ist.

Allerdings war auch hier eine Beschrïnkung wegen her groBen Ausdehnung des Ciebietes geboten; die Auswahl des Stoffes lïBt das Inhaltsverzeichnis erkennen. Insbesondere wurden die mathemati- 
schen Zeicheninstrumente trotz darauf bezüglicher Vorarbeiten ausgeschieden, da sie den Umfang des Buches nahezu verdoppelt hätten.

Der Verfasser hat sich einer vielseitigen Unterstïtzung erfreut, für die er allen beteiligten Herren seinen lebhaften Dank auszusprechen sich erlaubt. Durch das freundliche Entgegenkommen der Verfertiger und Besitzer von Instrumenten war es ihm vergönnt, auch solche Konstruktionen, die er nicht durch eigene Anwendung kannte, in Augenschein zu nehmen. Im Deutschen Museum in München wurde es ihm in entgegenkommender Weise ermöglicht, auch iiltere Instrumente kennen zu lernen. Leider fehlt in Deutschland eine dauernde Ausstellung, die eine Besichtigung und womöglich Erprobung der neuen Instrumente erlaubte.

Zu vicht geringem Danke ist der Verfasser den Herren Autoren, Verlegern und Konstrukteuren verpflichtet, die Abhandlungen, Abbildungen und Klischees gütigst zur Verfügung gestellt haben. Dem Vorteil gegenüber, da $B$ vielfach Originalabbildungen wiedergegeben werden, kommt der geringe Nachteil kaum in Betracht, daB die Bezeichnungen nicht iiberall dem Texte angepaßt werden konnten.

Endlich gedenkt der Verfasser mit besonderem Danke derer, die ihm bei der Drucklegung mit Rat und Tat zur Seite standen, unter ilinen besonders der Herren Dr. Schweydar und Prof. Dr. Peters.

Potsdam, im Juli 1912.

A. Gialle. 


\section{Inhaltsverzeichnis.}

\author{
I. Abschnitt.
}

\section{Arithimetische Apparate mit} logarithmischer Skala 1-22

1. Logarithmischer Rechenschieber

2. Bestandteile nach ihrer geschichtlichen Entwicklung. . . . . . . 3

3. Teilungen . . . . j

4. Stellenzahl . . . . . 9

5. Theorie ... . 10

6. Das Rechnen mit dem Stabe 12

7. Einige besondere Rechenschieber . . . . . . 16

8. Instrumente ron anderer Form ...... 21

II. Abschnitt.

Rechenmaschiuen . 23-48

9. Vorzüge des Rechnens mit Rechenmaschinen . . . :3

10. Zählwerk . . . . . . 23

11. Zebnerübertragung . . 24

12. Schaltwerk . . . . 25

13. Einteilung der Rechenmaschinen

14. Thomas - Burkhardtsche Maschine . $\therefore 8$

15. Divisionsmaschine Mercedes-Euclicl

16. Odhners Arithmometer.

17. Rechenmaschine Gau B

18. Sellings Maschine.

19. Multiplikationsmaschine Millionär.

\section{政}

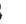

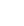

2

(3)

1


37. Theorie dieser Instru- Seite mente....... 71

38. Koordinatenplanimster . 77

39. Linearplanimeter . . . 87

40. Polarplanimeter . . . . 99

41. Liompensationsplanimeter $\mathbf{1 1 0}$

42. Verschiedene andere Planimeter mit Integrierrolle 111

43. Momentenplanineter . . 118

44. Schneidenplanimeter und Abarten . . . . . . 122

VIII. Abschnitt.

Apparate zur harmo-

nischen Analyse.131-154

45. Allgemeines und Verwendung des Planimeters . 131

46. Analysatoren von Yule . 134

47. Apparat von Le Conte . 137

48. Analysator von W.'Thomson...... 138
49. Analysator von HenriciCoradi . . . . . 140

50. Analysator von Sharp . 143

51. Iräzisionsinstrument von Sommerfeld und Wiechert 145

52. Analysator von Michelson und Stratton. . . . 148

53. Apparat von Terada zur Analyse und Synthese ron Schwingungen . . . 152

IX. Abschnitt.

Integraphen * 154-178

54. Grundlagen . . . . 154

55. Instrument von AbrlankAbakanowiez . . . . 157

56. Integrator von Paseal. . 161 Polarintegraph . . . 171

57. Integratoren für Differentialgleichungen . . . 172 
I. Abschnitt.

\section{Arithmetische Apparate mit logarithmischer Skala.}

1. Logarithmischer Rechenschieber. (Rechenstab, règle à calcul, règle logarithmique, slide rule, regola calcolatore). Eine Logarithmentafel läBt sich auf verschiedene Arten durch eine

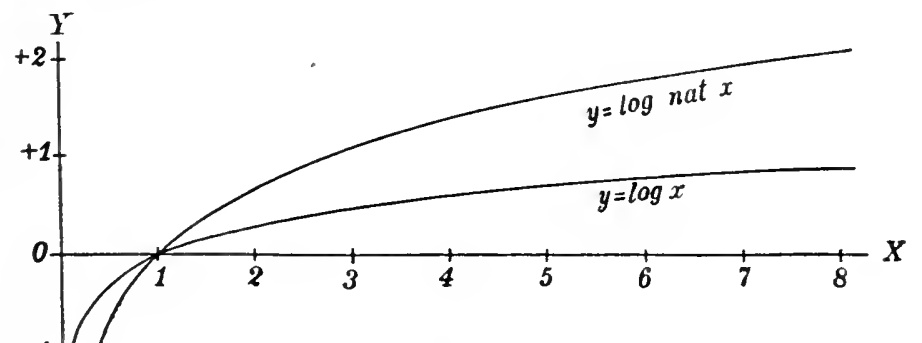

Fig. 1.

$-2$

graphische Darstellung ersetzen. Betrachtet man die Zahlen als Abszissen und die Logarithmen als Ordinaten in einem rechtwinkligen Koordinatensystem, so erhält man die logarithmische Kurve (curva logistica), mit deren Hilfe man die zueinander gehörenden Numeri und Logarithmen ablesen kann (Fig.1). Auch der Übergang von gemeinen (Briggschen) zu natürlichen (Napierschen) Logarithmen kann in der Weise erreicht werden, daB man beide logarithmischen Kurven für dieselben Abszissen darstellt; die Kurve der natürlichen Logarithmen zeichnet sich vor allen auf eine andere Grundzahl bezogenen dadurch aus, daB sie die Abszissenachse unter dem Winkel $\tau=45^{0}$ schneidet $^{1}$ ).

1) Für $y=\lg x$ ist nämlich $\frac{d y}{d x}=\operatorname{tg} \tau=\frac{1}{x}$, also für $x=\mathbf{1}(y=0)$ $\tau=45^{\circ}$. Im allgemeinen ist $\operatorname{tg} \tau=$ Modulus, also für Briggsche Logarithmen $(\operatorname{Mod}=0,43429) \tau_{B}=23^{\circ} 28^{\prime}, 5$. 
Wenn man in der allgemeinen Gleichung der Logistik $y=a \lg \frac{x}{a}$ in rechtwinkligen Koordinaten $r$ und $\varphi$ statt $x$ und $y$ schreibt, so

erhält man die Gleichung der logarithmischen Spirale $r=a e^{a}$ in Polarkoordinaten. Wenn die Polarwinkel $\varphi$ in arithmetischer Progression wachsen, so nehmen die zugehörigen Leitstrahlen in geometrischer Progression zu. Geht man also von einem Leitstrahl aus, der die Längeneinheit darstellt und schlägt mit ihm als Radius einen Kreis, so erhält man die Kreisbogenlängen als Logarithmen der durch ihre Endpunkte gelegten Leitstrablen der Kurve.

Ganz allgemein sind bei Zuordnung einer arithmetischen Reihe, die mit 0 anfängt, zu einer geometrischen Reihe, die mit 1 beginnt, die Glieder der ersteren die Logarithmen der Glieder der geometrischen Reihe und hierauf beruhte die Einrichtung der „ProgreBtabuln", der Vorläuferinnen der Logarithmentafeln. In Anlehuung an diese Tafeln nach Byrgi (Byrgius 1552-1632) und Napie ${ }^{1}$ ) (Lord Napier of Merchiston 1550-1617) entstand der ProgreBstab als erster Rechenstab.

Für gemeine Logarithmen entspricht z. B. einer arithmetischen Reibe mit der Differenz 0,1 eine geometrische mit dem Exponenten 1,259 (untere Seite der Figur 2 I). Die Strichintervalle sind die graphische Darstellung der Glieder der geometrischen Reihe (Potenzen von 10), während die den Teilstrichen beigefügten Zahlen (die Exponenten von 10) in arithmetischer Reihe wachsen. Liest man zu den Zahlen der arithmetischen Reihe (den Logarithmen) die etwa auf Millimeterpapier gezeichnete Skala ab, so erbält man durch Schätzung von Zehntelmillimetern die Numeri auf drei Stellen, und die Doppelskala, in der (oben in Figur 2 I) die runden Zahlen (Numeri) eingeschrieben sind, ersetzt eine Antilogarithmentafel.

Wenn man umgekehrt die Glieder der arithmetischen Reihe (Logarithmen) auf eine Skala aufträgt und nur die Teilung und Bezifferung der geometrischen Reihe beifügt, so kann man die Logarithmen abgreifen und graphisch addieren oder subtrahieren. Auf diese Weise erhält man eine Skala, mit der man logarithmische Operationen ausführen kann, olıne die Logarithmen zahlenmäßig zu kennen (oben in Fig. 2 II).

Um einen Ersatz für die Logaritlimentafel zu bekommen, muB

1) Oft Neper genannt. Über den Progreßstab vgl. C. H. Müller, ferner Kewitsch, Zeitschr. f. math. u. naturw. Unterricht, 1896, S. 333. 
man noch die Bezifferung und Teilung der aritbmetischen Reihe hinzufügen, wie sie unten in der Skala II der Figur 2 angegeben ist. Diese bei den eigentlichen Rechenschiebern als $L$-Skala bezeichnete Skala wird, wie wir sehen werden, verhältnismäBig selten in Anwendung kommen. In beiden Doppelskalen, I und II der Figur 2, stehen oben die Zahlen, unten die Logarithmen. Die erste (I) entspricht dem ProgreBstabe, die zweite (II) dem Rechenschieber.
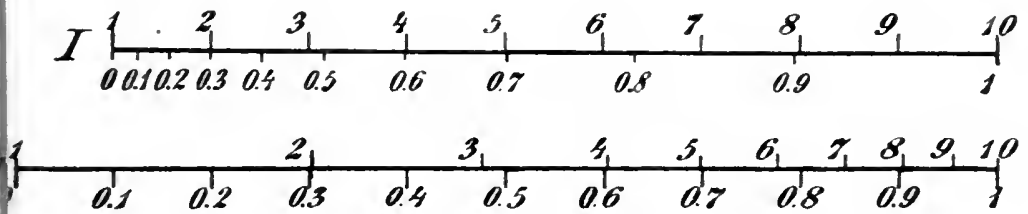

Fig 9 .

2. Bestandteile nach ihrer geschichtlichen Entwicklung. Die erste Form eines solchen Rechenstabes stammt von Gunter (1581-1626: Gunters scale oder Gunters line) und hat sich noch für besondere Zwecke als Navigation scale erhalten. Diese Skala erfordert die Verwendung eines Zirkels, dessen Spitzen in Löcher auf Messingplättchen eingesetzt werden. Man greift die Längen der Logarithmen ab und setzt sie additiv oder subtraktiv aneinander. Die so erhaltene Länge trägt man rom Anfangspunkt ab, und kommt so gemäb den Regeln $\log a+\log b=\log (a \cdot b)$ und $\log a-$ $\log b=\log \left(\frac{a}{b}\right)$ auf die Zahl $a \cdot b$ oder $\frac{a}{b}$, welche das Produkt oder den Quotienten der zuerst abgegriffenen Zahlen darstellt. Weil man aber zuweilen über die eigentliche Skala hinwegspannen muB, so ist ein Verlängerungsbrettchen nnentbehrlich. Um den Zirkel zu vermeiden, führte Oughtred $\left.(1574-1660)^{1}\right)$ den Doppelstab ein, der zwei vollständig gleiche, aneinander verschiebbare Skalen besaB. Die erste gröBere technische Ausführung wurde auf Anregung von James Watt um 1770 in der Metallwarenfabrik in Soho verwendet (Soho Rules). Um 1650 gab Seth Patridge dem Stabe die noch heute iibliche Gestalt durch Einfügung der Zunge (des Schiebers), die in Felgen des eigentlichen Stabes gleitet,

1) Bisher wurde dies Wingate $(1593-1656)$ zugeschrieben, obige Angabe macht $\mathrm{Hammer}$ (Zeitschr. f. Vermessungsw., 40, S. 27). 
wodurch eine sichere Führung der Skalen aneinander erreicht wird. Auf diese Weise war für vier Skalen Raum vorhanden, wobei die obere Stab- $(A)$ und die obere Zungenteilung $(B)$ gleich waren, ebenso die untere Zungenteilung $(C)$, während die untere Teilung (D) auf dem Stabe den doppelten MaBstab besaB. Durch die Firma Tavernier ${ }^{1}$ ) in Paris wurden die je zwei aneinauder liegenden Skalen (Fig. 3) genau gleich $(A=B$ und $C=D)$ und zwar $C$ und $D$ in doppeltem Maßstab wie $A$ und $B$ ausgeführt ${ }^{2}$ ), so daB die Zahlen $A$ und $B$ die Quadrate der darunter stehenden Zahlen $C$ und $D$ sind, also ein bequemes Hilfsmittel zum Quadrieren und Quadratwurzel-Ausziehen bieten. Zuerst wird die Hinzufügung einer quadratischen Skala von Lambert ${ }^{3}$ ) erwähnt.

Um nun die Übertragung zwischen nicht aneinander liegenden Skalen zu ermöglichen, oder sicherer zu gestalten, kam der von Mannheim ${ }^{4}$ ) 1851 selbständig erfundene, aber auch schon früher benutzte Läufer (courseur) hinzu, der auch zum Festhalten einer Einstellung bei Verschiebung der Zunge wichtige Dienste leistet. Er wird entweder als ein verschiebbares Metallstïck mit zwei gleichen längs der Fugen zwischen $A$ und $B$, bzw. $C$ und $D$ binstreifenden, zugespitzten oder geschärften Zeigern ausgeführt oder in neuerer Zeit in Form eines an zwei oder allen vier Seiten eingefaßten Glasfensters konstruiert, das senkrecht zu den Skalen, also parallel den Teilstrichen einen (oder zwei) auf der Unterseite des Glases eingeritzten Strich trägt (Fig. 3). Durch Federn wird eine sichere Führuug des Läufers gewährleistet. Für genauere Ablesung und für schwächere Augen werden auch Läufer mit Lupe oder Zylinderlinse ${ }^{5}$ ) gefertigt. Als nach dem deutschfranzösischen Kriege auf Anregung von Prof. Goering $\left.{ }^{6}\right)(1841-1906)$ Dennert \& Pape in Altona die Verfertigung der aus Frankreich nicht mehr erhältlichen Rechenschieber unternahmen, wurde die wichtige Neuerung, die Teilungen auf Zellhorn (Zelluloid) anzubringen, eingeführt,

1) Anfangs Gravet-Lenoir, dann Tavernier-Gravet, zuletzt Tavernier-Vinay.

2) Figur 3. Im übrigen empfiehlt es sich, den folgenden Erörterungen an der Hand eines Rechenschiebers zu folgen.

3) Beschreibung und Gebrauch der logarithmischen Rechenstäbe. Augsburg 1761 und 1772.

4) Artillerieoffizier in Metz, später Professor in Paris.

5) Nestler in Lahr (Baden).

6) Anleitung zum Gebrauch des Rechenstabs. Gebr. Wichmann in Berlin. 
während sie vorher direkt auf Holz, Elfenbein oder Metall aufgetragen waren. Die Bekleidung der Ober- und Unterseite des Stabes mit Zellhorn ${ }^{1}$ ) dient zur Verhinderung der Krümmung durch Feuchtigkeit; zur Regulierung des Ganges der Zunge werden Stellschrauben ${ }^{2}$ ) angewendet. Auf diese Weise ist ein nahezu einheitlicher Typus der Rechenschieber aus den ersten Fabriken Deutschlands ${ }^{3}$ ) entstanden. Die gebräuchlichste Länge beträgt $25 \mathrm{~cm}$ oder wenige Zentimeter mehr. Die halbe und doppelte Länge kommen ebenfalls vor, erstere bei Tascheninstrumenten ${ }^{4}$ ) ron geringerer Genauigkeit, letztere an der Grenze der Handlichkeit.

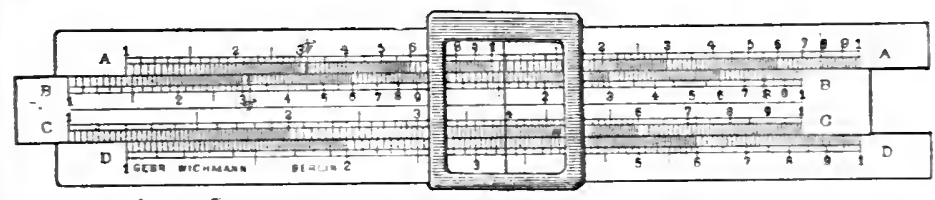

Fig. 3.

3. Teilungen. Die unteren Skalen $C$ und $D$ enthalten die logarithmischen Längen für die Zahlen 1 bis 10 . Diese Längen ${ }^{5}$ ) sind von den Anfangspunkten aus abgetragen und an den Teilstrichen sind die Zahlen angeschrieben. Nur an Stelle ron 10 steht wieder 1, da der dekadische Logarithmus ron 1 und 10 nur durch die Kennziffer unterschieden wird. Da nun die Abstände sich verringern, so war für die Unterabteilungen maBgebend, da $B$ die Intervalle nicht mehr als $1 \mathrm{~mm}$ und nicht weniger als $0,5 \mathrm{~mm}$ betragen sollen, um die Ablesung möglichst genau, aber die Teilung nicht zu eng zu machen. Infolgedessen konnte (bei $25 \mathrm{~cm}$

1) S. Note 5 S. 4.

3) Wir erwähnen noch A. W. Faber.

4) Figur 3 .

5) Zahl. Logarithmus $x \lambda=$ Länge

\begin{tabular}{|c|c|c|}
\hline 1 & 0,00000 & $\times 250=$ \\
\hline 2 & 0,30103 & $\times 250=75,26$ \\
\hline 3 & 0,47712 & $\times 250=119,28$ \\
\hline 4 & 0,60206 & $\times 250=150,52$ \\
\hline 5 & 0,69897 & $\times 250=174,74$ \\
\hline 6 & 0,77815 & $\times 250=194,54$ \\
\hline$\pi$ & 0,84510 & $\times 250=211,28$ \\
\hline 8 & 0,90309 & $\times 250=225,77$ \\
\hline 9 & 0,95424 & $\times 250=238,56$ \\
\hline 10 & 1,00000 & $\times 250=250,00$ \\
\hline
\end{tabular}


Länge der Skala) der Zwischenraum zwischen 1 und 2 in 100 Teile geteilt werden. ${ }^{1}$ ) Von 2 bis 4 schreiten die Zahlen um 0,02 , zwischen 4 und 10 um 0,05 fort. Um die Übersicht nicht zu erschweren, ist bei den Zwischenstrichen (außer bei den Zehnteln von 1 bis 2) die Bezifferung fortgelassen und sind nur längere Striche zur Unterscheidung benutzt worden. Die an einander liegenden Skalen $A$ und $B$ der oberen Hälfte des Stabes und der Zunge sind ganz entsprechend wie $C$ und $D$ konstruiert, nur bestehen sie aus zwei halb so langen $(125 \mathrm{~mm})$ einander fortsetzenden, unter sich gleichen Skalen. Man kann daher den Anfangsstrich als 1, den Mittelstrich als 10, den Endstrich als 100 ansehen. Jedoch sind alle drei mit 1 bezeichnet. Der Kürze der Teilungen wegen sind hier die Unterabteilungen an Zahl geringer, indem sie zwischen 1 und 2 um 0,02, zwischen 2 und 5 um 0,05 , zwischen 5 und 10 um 0,1 fortschreiten. AuBer diesen Zwischenstrichen sind auf den meisten Rechenschiebern noch einige Striche eingetragen, die für besondere $\mathrm{Zwecke}$ nützlich sind, oft auch störend empfunden werden, so für $\pi=3,142$ (zugleich zur Verwandlung preuBischer $\mathrm{FuB}$ in Meter ${ }^{2}$ ) dienend), $\varrho^{\prime \prime}=206265$, $o^{\prime}=3438$ für alte Kreisteilung, $\varrho_{\prime \prime}=636620$ für neue Kreisteilung u. a. Die Rückseite der Zunge trägt ebenfalls Teilungen, die entweder bei umgewendet eingeschobener Zunge an den Vorderteilungen oder bei umgewendetem Instrument an Indexstrichen in zwei Ausschnitten der Unterfl̈̈che des Stabes links und rechts abgelesen werden. Die obere dieser Teilungen auf der Rückseite der Zunge, mit $S$ bezeichnet, gibt die Sinus in der Einheit der $A$-Teilung, die untere, mit $T$ bezeichnete die Tangenten der Winkel in der Einheit der $D$-Teilung, wäbrend die mittlere, meist $L$ benannte, eine um $0,5 \mathrm{~mm}$ gleichmäBig fortschreitende ist. Schiebt man die Zunge ungewendet ein, so daB die $S$ - und $A$-Teilung an

$$
\begin{aligned}
& \text { 1) Zahl. Logarithmus } \times \lambda=\text { Länge. Diff. } \\
& \begin{array}{lll}
1,00 & 0,00000 & \times 250=0,00 \mathrm{~mm} \\
1,01 & 0,00432 & \times 250=1,08
\end{array} \\
& 1,02 \quad 0,00860 \times 250=2,15, " 1,07 \\
& \begin{array}{lllll}
1,98 & 0,29667 & \times 250=74,17 \quad " & 0,54 \\
1,99 & 0,29885 & \times 250=\mathbf{7 4 , 7 1} \quad " & 0,55
\end{array} \\
& 2,00 \quad 0,30103 \times 250=75,26 " 0,55
\end{aligned}
$$


einander liegen und Anfangs- und Endstrich gegenübersteben, so sind die Zahlen $A 1$ bis $A 100$, mit 100 dividiert, die Sinus der auf der $S$-Skala angegebenen Winkel. Diese beginnen mit $0^{0} 34^{\prime}, 4^{1}$ ), für kleinere Winkel rechnet man mit dem Arcus-Verbältnis $\rho^{\prime}$, nämlich $\sin \alpha=\frac{\alpha^{\prime}}{3438}$ in Minuten. Bis zu $10^{\circ}$ schreitet die $S$-Teilung um $5^{\prime}$, bis $20^{\circ}$ um $20^{\prime}$, von $20^{\circ}$ bis $40^{\circ}$ um $30^{\prime}$, von $40^{\circ}$ bis $70^{\circ}$ um $1^{\circ}$ fort, diesem letzten bezifferten Striche folgen die Striche bis $80^{\circ} \mathrm{im}$ Abstande von $2^{0}$, dann noch $85^{\circ}$ und $90^{\circ}$. Da die $B$-Teilung der Zunge identisch mit der $A$-Teilung des Stabes ist, so entsprechen sich auch die Striche $B$ und $S$ auf der Vorder- und Rückseite der Zunge und da der obere ${ }^{2}$ ) Indexstrich im rechten Ausschnitt der Unterfläche mit dem Endstrich der Stabteilung auf der Oberseite korrespondiert, so liest man auf der nach rechts ${ }^{2}$ ) ausgezogenen umgewendeten Zunge die Grade der $S$-Skala unter dem Endstrich $\mathrm{ab}$, deren Sinus auf der $B$-Skala bei umgewendetem Instrument am Indexstrich der Unterseite erscheinen. Wenn man die Zunge in der Weise umgekehrt einschiebt, $\mathrm{da} B$ die $C$-Teilung gegenläufig an $\operatorname{der} A$-Teilung, ebenso $B$ an $D$ anliegt, und die Zunge nach links herauszieht, so kann man am Indexstrich des linken Ausschnittes der Unterseite die Grade ${ }^{3}$ ), deren Ziffern auf dem Kopfe stehen, auf der $S$-Skala einstellen. Da diesem Indexstrich auf der Oberseite der Anfangsstrich der Stabteilung ( $A$ und $D$ ) entspricht, so steht hier auf der $B$-Skala gegenüber dem Anfangsstrich von $D$ der Sinus. Oder man kaun, da das herausgezogene Stück der Zunge der Einrückung am rechten Ende gleich ist, ebenso gut dieselbe Zahl auf $A$ über dem Endstrich $C$ der Zunge ablesen, da ja $A$ und $B$ identische Skalen und in dieser Lage gegenläufig sind. Bei normaler Zungenstellung endlich, $d . h$. bei gleich gerichteten und an einanderliegenden $A$ und $B$ erhält man beim Ausziehen der Zunge nach rechts zu den am Index der Unterseite abgelesenen Gradzahlen $S$ über dem Anfangsstrich von $B$ (auf der Zunge) auf der Stabteilung $A$ die reziproken Werte der Sinus, also

1) $\sin 0^{0} 34^{\circ}, 4=0,01$, dem mittleren Stich $A 10$ entspricht $\sin 5^{\circ} 44^{\prime}, 3=0,1$, dem Endstrich $A 100$ entspricht $\sin 90^{\circ}=1$.

2) Links und rechts sind stets so zu verstehen, daB der Anfangspunkt der Stabteilungen ( $A$ und $D$ ) links liegt, der Endpunkt rechts. Bei umgewendetem Instrument ist dann die der Skale $D$ entsprechende Seite die obere, die der Skale $A$ entsprechende die untero.

3) Z. B. $S 30^{\circ}, B 50: 100=0,5$. 
die Kosekanten. ${ }^{1}$ ) Denn es ist jede Zahl $A$ über dem Anfangsstrich der Zunge $(B 1)$ reziprok zu jeder Zahl $B$ unter dem Endstrich $\left(A\right.$ 100) des Stabes ${ }^{2}$, und andererseits liegen die entsprechenden Zahlen $B$ der Vorderseite und $S$ der Rückseite der Zunge übereinander.

Die $T$-Teilung entspricht den $C$ - und $D$-Skalen, so da $B$ bei umgewendeter Zunge und Anliegen von $T$ und $D$ beim Zusammenfallen der Endstriche die Tangenten ${ }^{3}$ ) direkt unter den Graden stehen. Dem Anfangsstrich $5^{0} 43^{\prime}, 8$ von $T$ entspricht die Ablesung 0,1 auf $D$, dem Endstrich $45^{0} D$ 1. Die $T$-Teilung schreitet bis $20^{\circ}$ um $5^{\prime}$, von $20^{\circ}$ bis $45^{\circ}$ um $10^{\prime}$ fort. Für. Winkel kleiner als $5^{0} 43^{\prime}, 8$ wird tang durch sin ersetzt. Für Winkel gröBer als $45^{\circ}$ wird $\operatorname{tg} x=\frac{1}{\operatorname{tg}\left(90^{\circ}-x\right)}$ gebildet. Die Regeln sind entsprechend denen bei der $S$-Skala, so daB wir sie kurz aufstellen:

Vorderseite $T$ an $D$, Rückseite Index links: Für Winkel $T$ gegenüber $D 1$ ist $C$ gegenüber Index die Tangente.

Vorderseite $A, B, C, D$ in normaler Lage: $C$ gegenüber $D 1$ ist T'angente von $T$ am linken Index der Rückseite oder auch $D$ gegenüber $C 10$ Kotangente desselben Winkels.

Vorderseite $A, C, B, D$ bei umgekehrt eingeschobener Zunge: $D^{4}$ ) gegenüber $B$ 1. Tangente von $T$ (Ziffern auf dem Kopfe) am oberen Index rechts.

Die $L$-Teilung trägt eine den andern Skalen entgegengesetzt laufende gleichmäBige Teilung und Bezifferung von 0 bis 10 mit 50 Unterabteilungen zwischen je 2 Zahlen. Wendet man und dreht man gleichzeitig die Zunge um, so daß $L$ in gleicher Richtung wie $A$ und $D$ läuft, so kann man mit Hilfe des Läufers zu jeder Zahl $D$ den (beim Zusammenfallen der Endstriche) darüberstehenden Logarithmus (auf dem Kopfe stehend) auf $L$ ablesen. Bequemer liest man bei normaler Zungenlage und nach rechts herausgezogener Zunge am rechten unteren Index der Unterseite den Logarithmus der Zahl $D$ ab, die dem Anfangsstrich $(C 1)$ der Zunge gegenübersteht. ${ }^{5}$ )

1) $\mathrm{Zu} S 30: A 2$ über $B 1: \operatorname{cosec} 30^{\circ}=2$.

2) $\log A+\log B=\log 1$.

3) Hier muB man die abgelesenen Zahlen mit 10 dividieren.

4) Oder auch $C$ (auf dem Kopfe stehend) gegenüber $\boldsymbol{A} \mathbf{1 0 0 .}$

5) Denn das rechts herausgezogene Stück ist gleich dem eingeschobenen links, also sind die vom Endpunkt des ersteren gemessenen 
Zur Verwendung des Rechenschiebers als Zeicheninstrument dient die abgeschrägte obere Kante (neben der $A$-Skala) mit einer $25 \mathrm{~cm}$ langen Millimeterteilung, deren Endstriche mit denen der Stabteilungen zusammenfallen. Die gerade Kante des Instrumentes (an der $D$-Skala) ist dagegen ihrer ganzen Länge nach (der auch die Länge der Zunge gleich ist) in Millimeter geteilt. Zieht man die Zunge heraus, so setzt sich ihre Länge an der dadurch sichtbar werdenden Bodenfläche fort, die also mit der Zungenlänge zusammen einen $\mathrm{MaBstab}$ von doppelter Länge des Instruments (etwa $50 \mathrm{~cm}$ ) darstellt.

4. Stellenzahl. Für alle Rechnungen mit dem $(25 \mathrm{~cm})$ Rechenschieber zieht man höchstens 4 Ziffern der vorgelegten Zahlen ohne Rücksicht auf die Stellung des Kommas in Betracht. Die Stellung des Kommas im Resultat erhält man am besten durch besondere Überlegung ${ }^{1}$ ).

In manchen Fällen, besonders bei einer größeren Anzahl aufeinanderfolgender Multiplikationen und Divisionen, kann es rorteilhaft sein, die Stellung des Kommas im Resultat durch eine mechanische Regel festzustellen oder zu kontrollieren. $\mathrm{Zu}$ diesem Zwecke denkt man sich die gegebenen Zahlen in der Form $a 10^{n}+b 10^{n-1}+c 10^{n-2}+\cdots+g 10^{1}+h 10^{0}+i 10^{-1}+k 10^{-2} \ldots^{2}$ ). Den Exponenten $n$ bezeichnet man als führende Ordnungsziffer. Bei einem Produkt ist die führende Ordnungsziffer gleich der Summe der führenden Ordnungsziffern der Faktoren. ${ }^{3}$ ) Reicht die Skala nicht zur Aneinandersetzung der Logarithmen aus, so ist ein Rückschlag nötig, indem man den Endstrich der Zunge statt ihres Anfangsstriches benutzen muB, was einer Division mit 10 gleichkommt. Man muß daher das Resultat mit 10 multiplizieren oder für jeden Rückschlag die Ordnungsziffer um 1 erhöhen. ${ }^{4}$ ) Bei der Division ist die führende Ordnungsziffer des Quotienten

Logarithmen den vom Anfangspunkt des letzteren gezählten Zahlen zugehörig. Am rechten Ausschnitt der Unterseite befinden sich zwei Indizes.

1) $0,000383 \times 0,00282$. Der Rechenschieber gibt $383 \times \mathbf{2 8 2}=108$, wenn das Komma nicht beachtet wird. Es ist nun in Einheiten der 3. Dezimalstelle $0,4 \times 3$ zu bilden, man erhält also ungefähr eine Einheit der 6. Dezimalstelle im Resultat: 0,00000108 .

2) $1536,749=1 \cdot 10^{3}+5 \cdot 10^{2}+3 \cdot 10^{1}+6 \cdot 10^{0}+7 \cdot 10^{-1}+4 \cdot 10^{-2}$ $+9 \cdot 10^{-3}$. Führende Ordnungsziffer 3 .

3) $2 \times 3=6,0+0=0 . \quad 516 \times 16=8256,2+1=3$.

4) $3 \times 4=(0,3 \times 4) 10=12,(0+0)+1=1$. 
gleich der Differenz der Ordnungsziffern von Dividend und Divisor, wenn man mit derselben Skala ausreicht. ${ }^{1}$ ) Ist dagegen ein Vorschlag erforderlich, der eine Ablesung am Endstrich statt am Anfangsstrich, also eine Multiplikation mit 10 bedeutet, so muB man die Ordnungsziffer um 1 erniedrigen. ${ }^{2}$ ) Diese Regeln werden gewöhnlich in den Gebrauchsanweisungen in anderer Form ausgesprochen, indem man die Stellenzahl (die um 1 höher ist als die Ordnungsziffer) einführt.

Multiplikation:

Ergebnis in derselben Teilung rechts: Stellensumme minus 1 Ergebnis in derselben Teilung links (bei $A / B$ auch in der folDivision: genden Teilung rechts): Stellensumme.

Ergebnis in derselben Teilung links: Stellendifferenz plus 1 Ergebnis in derselben Teilung rechts (oder bei $A / B$ in der vorhergehenden links): Stellendifferenz.

Zur Erinnerung an diese Regeln findet sich auf manchen Stäben linker Hand $Q+1$, rechter Hand $P-1$ angeschrieben ( $Q$ Quotient, $P$ Produkt).

5. Theorie. Die Striche $x$ einer Skale von der Länge $d$ seien die Endstriche der vom Anfangsstrich $x_{0}$ abgetragenen Strecken $\lambda \varphi(x)$. Wenn noch der Endstrich der Skala mit $x_{n}$ bezeichnet wird, so ist $\lambda \varphi\left(x_{0}\right)=0$ und $\lambda \varphi\left(x_{n}\right)=\lambda$, also $\varphi\left(x_{0}\right)=0$ und $\varphi\left(x_{n}\right)=1 .^{3}$ ) Die Länge einer zweiten Skala werde entsprechend mit $\mu$, Anfangs- und Endstrich mit $y_{0}$ und $y_{n}$ bezeichnet und die Streckenlängen vom Anfangsstrich aus seien $\mu \chi(y)$, dann ist ebenso $\chi\left(y_{0}\right)=0$ und $\chi\left(y_{n}\right)=1$.

Bei beliebiger Verschiebung der Skalen und gleichlaufender

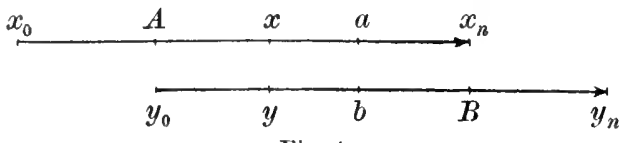

Fig. 4.

Teilung seien $(x, y)$ und $(a, b)$ zwei Paare einander gegenüberliegender Striche. Ferner liege dem äußersten Strich $y_{0}$ der Strich $A$, dem äußersten Strich der andern Skala $x_{n}$ der Strich $B$ gegenüber. Wir lesen aus Figur 4 ab:

1) $6: 3=2,0-0=0 . \quad 8256: 16=516,3-1=2$.

2) $12: 4=(1,2: 4) \times 10=3,(1-0)-1=0$.

3) Für $\varphi(x)=\log x$ ergibt sich $x_{0}=1, x_{n}=10$; für $\varphi(x)=$ $\log (10 \operatorname{tang} x)$ ist $x_{0}=5^{0} 43^{\prime}, 8, x_{n}=45^{\circ}$. 
oder

$$
\lambda[\varphi(a)-\varphi(x)]=\mu[\chi(b)-\chi(y)]
$$

$$
\lambda \varphi(x)-\mu \chi(y)=\lambda \varphi(a)-\mu \chi(b) .
$$

Lassen wir das Paar $(x, y)$ wandern, so da $\mathrm{B}$ es einmal mit

$$
x=A, y=y_{0},
$$

das zweite Mal mit

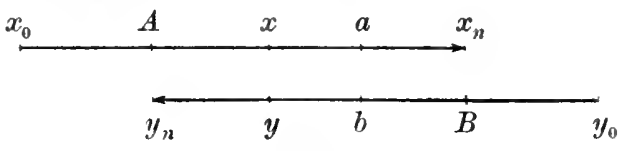

Fig. 5. $x=x_{n} ; y=B$ zusammenfällt, so erhalten wir

$$
\lambda \varphi(\boldsymbol{A})=\lambda \varphi(a)-\mu \psi(b)=\lambda \varphi(x)-\mu \psi(y)=\lambda-\mu \%(B) .
$$

Sind die Skalen gegenläufig (Figur 5), so ist

oder

$$
\lambda[\varphi(a)-\varphi(x)]=-\mu[\chi(b)-\chi(y)]
$$

und

$$
\lambda \varphi(x)+\mu \chi(y)=\lambda \varphi(a)+\mu \chi(b)
$$

(2) $\lambda \varphi(A)+\mu=\lambda \varphi(a)+\mu \chi(b)=\lambda \varphi(x)+\mu_{\chi}(y)=\lambda+\mu \chi(B)$.

Die Ausdrücke $\lambda \varphi(x) \mp \mu \chi(y)$ haben also für eine bestimmte gegenseitige Lage der Skalen einen konstanten Wert, der durch die dem Anfargs- oder Endstrich der andern Skala gegenüberliegende Ablesung bestimmt ist. Faßt man (1) oder (2) als eine Gleichung zwischen zwei Unbekannten $x$ und $y$ auf, so kann man, wenn noch eine zweite Gleichung zwischen ihnen gegeben ist, (falls sie einfacher Natur ist) mit dem Rechenstab die Unbekannten finden. Ein solcher Fall ist der, daB die Summe oder Differenz $x \pm y=k$ gegeben ist. Man sucht dann dasjenige Zahlenpaar bei der durch die erste Gleichung (1 bzw. 2) bestimmten Skalenstellung auf, das die Summe oder Differenz $k$ hat. ${ }^{1}$ )

1) Ein Beispiel bietet für den gewöhnlichen Rechenschieber die Auflösung einer quadratischen Gleichung, z. B. $7,15 x^{2}+36,33 x$ $+40,45=0$. Durch Division (mittels des Rechenschiebers) mit 7,15 erhält man $x^{2}+5,09 x+5,66=0$. Zwischen den beiden Wurzeln $x_{1}$ und $x_{2}$ bestehen also die Gleichungen: $x_{1} \cdot x_{2}=+5,66$ oder $\log x_{1}$ $+\log x_{2}=\log (5,66)$, und $x_{1}+x_{2}=-5,09$. Nach (2) schiebt man die Zunge gegenläufig ein, stellt ihren Anfangsstrich über 5,66 der Stabteilnng $D$ und findet mit Hilfe des Läufers auf den nicht anliegenden Skalen $C$ und $D$ die Zahlen 1,63 und 3,46 gegenüberstehend, deren Summe 5,09 ist, also $x_{1}=-1,63, x_{2}=-3,46$. - Bei normal 
Die Gleichungen (1) und (2) enthalten die Lösung der für den Rechenschieber vorkommenden Aufgaben, wenn sie den besonderen Fällen angepaßt werden. Insbesondere wird häufig $\mu=\lambda$ sein. ${ }^{1}$ )

6. Das Rechnen mit dem Stabe.

Bei zwei identischen Skalen ( $A$ und $B$ oder $C$ und $D$ ) ist nach Figur 4 und nach 5. (1) bei einer bestimmten Stellung $\log A+\log b=\log a$ also $A b=a$. Hieraus ist das Verfahren bei der Multiplikation ersichtlich, wo die Reihenfolge der Faktoren willkürlich ist. Man sieht zugleich, daß bei dieser Stellung alle Zahlen $x$ das $A$-fache aller Zahlen $y$ sind, der Rechenschieber vertritt daher eine Verwandlungstabelle, die man leicht durch eine andere (wie es z. B. für Kurstafeln wünschenswert ist) ersetzen kann, indem man $A$ einen andern Wert gibt (durch Verschiebung der Zunge). Andrerseits ist $\log a-\log b=\log A$ oder $a / b=A$, woraus sich das Verfahren für die $\mathrm{D}$ i vision ergibt. Zugleich ist für jedes Wertepaar $x / y=A$; wenn die Fuge zwischen den beiden Teilungen als Bruchstrich betrachtet wird, so ist der Bruch, den jedes Zahlenpaar bildet, für eine bestimmte Zungenstellung derselbe.

Hiermit ist die wichtigste Anwendung des Stabes, nämlich die für die Proportionalrechnung, gegeben. Es ist $x: y=a: b$. Ist $x$ gesucht, so stellt man $a$ über $b$ und liest $x$ gegenüber $y$ ab.

Bei identischen, aber gegenläufigen Skalen ist $x \cdot y=A$ nach 5. (2) und Figur 5. In dem Falle $x_{0}=A$ (d. h. wenn sich $x_{0}$ und $y_{n}$ gegenüberstehen, die Zunge ganz eingeschoben ist), sind $x$ und $y$ reziproke Werte (weil $x_{0}=1$ ), die mit dem Läufer eingestellt werden, weil die Skalen nicht aneinander liegen.

Die Quadratbildung erfolgt entweder durch Multiplikation

eingeschobener Zunge, bildet man nach (1) $\log (5,66)-\log x_{2}=\log x_{1}$. Man hält in diesem Falle mit dem Läufer die Zahl 5,66 auf der Stabteilung fest und verschiebt die Zunge so lange, bis der ihrem Anfangsstrich entsprechende Wort $\left(x_{1}=A\right)$ der Stabteilung und die unter dem Läuferstrich auf der Zunge erscheinende Zahl $\left(y=x_{2}\right)$ die Summe 5,09 ergeben. - Lösung der Keplerschen Gleichung. Astr. Nachr. 186, 357 .

1) Beim gewöhnlichen Rechenschieber ist für die Skalen $A$ und $B$ $\lambda=\mu$ und ebenso für $C$ und $D$. Wenn man dagegen $A$ oder $B$ mit $C$ oder $D$ kombiniert, so ist $\lambda$ (für $A$ und $B$ ) $=1$ gesetzt, $\mu=2$ (für $C$ und $D)$. In diesem Falle ist $(A$ oder $B): \varphi(x)=\log x,(C$ oder $D$ : $2 \chi(y)=2 \log y=\log y^{2}$. Wenn die Anfangspunkte zusammenfallen, erhält man daher $x$ (auf $A$ oder $B$ ) $=y^{2}$ (auf $C$ oder $D$ ) oder auch $y=\sqrt{ } x$. 
oder einfacher durch Übergang von der Skala $D$ auf $A$ oder von $C$ auf $B$ mit Hilfe des Läufers. Die Quadratwurzel wird umgekehrt durch Übertragung von $A$ auf $D$ oder von $B$ auf $C$ gefunden; man teilt die gegebene Zahl rom Komma in Gruppen von je zwei Stellen. Ist die Zahl ungeradstellig, so benutzt man die linke, ist sie geradstellig, die rechte Hälfte von $A$ (oder $B$ ).

Den Kubus $N^{3}$ findet man, indem man den Zungenanfangsstrich über $N(D)(N$ auf Skala $D)$ stellt und $N^{3}$ über $N(B)$ auf $A$ abliest. Bei umgekehrt eingeschobener Zunge stellt man $N(A)$ und $N(C)$ einander gegenüber und findet $N^{3}$ auf $A$ gegenüber $1 C(1$ auf Skala $C$ ). Da nur ein Endstrich in Frage kommt, entsteht kein Zweifel. Bei der Kubikwurzelziehung teilt man die gegebene Zahl vom Komma aus in Gruppen von drei Ziffern. Je nachdem die erste Gruppe gültiger Ziffern ein-, zwei- oder dreistellig ist, benutzt man die linke Hälfte von $A$ und den Anfangsstrich von $B$ (für 1-10), die rechte Hälfte von $A$ und den Anfangsstrich von $B$ (für 10-100), oder die rechte Seite von $A$ und den Endstrich ron $B$ (für Zahlen 100-1000). ${ }^{1}$ ) Bei verkehrt eingeschobener Zunge stellt man einen Endstrich der nach links fortschreitenden Skala $C$ auf die Zahl $N$ der anliegenden Skala $A$ und zwar stellt man, je nachdem die erste dreistellige Gruppe außer etwa roranstehenden Nullen

1 Ziffer enthält:

$N$ auf der linken Hälfte von $A$ : den rechten Endstrich ron $C$

2 Ziffern enthält:

$N$ auf der rechten Hälfte von $A$ : den rechten Endstrich von $C$

3 Ziffern enthält:

$N$ anf der linken Hälfte von $A$ : den linken Endstrich von $C$

1) Beispiele: $\sqrt[3]{8}=2$. Läufer auf $8 A$ (links). Man rerschiebt die Zunge, bis $1 C$ über derselben Zahl $D$ steht, dic auf $B$ (links) unter dem Läuferstrich erscheint. (Also $1 C$ über $2 D$, wenn $2 B$ unter $8 A$ ). $\sqrt[3]{27}=3$. Läufer auf $27 A$ (rechts). Man verschiebt die Zunge, bis auf der linken Hälfte von $B$ dieselbe Zahl unter dem Läuferstrich steht, wie auf $D$ unter dem Zungenanfang. ( $C$ über $3 D, 27 A$ über $3 B$ ). $\sqrt[3]{216}=6$. Läufer auf $216 \mathrm{~A}$ (rechts). Zungenverschiebung bis auf $D$ unter dem Zungenende dieselbe Zahl steht, wie auf $B$ (rechts) unter dem Läuferstrich $\left(10 C\right.$ über $6 \mathrm{D}, 216 A_{r}$ über $6 B_{r}$ ). Ist $Y$ die Zahl auf $A$ und steht darunter $y$ auf $B$, so steht auf $A$ über dem Zungenendstrich $\frac{N}{y}=x^{2}$ und unter demselben Zungenende $x$ auf $D$, mithin ist $N=x^{3}$ für $y=x$. 
gegenüber $N$. Diejenige Zahl, die auf den anliegenden Skalen $A$ und $C$ sich selbst gegenübersteht, ist $\sqrt[3]{N}$.

Um eine beliebige Potenz oder Wurzel mit dem Rechenschieber zu bilden, muB man mit Benutzung der Formeln $\log \left(a^{n}\right)=n \log a$ und $\log (\sqrt[n]{a})=\frac{1}{n} \log a$ und $\log \log \left(a^{n}\right)=\log n+\log \log a$, $\log \log (\sqrt[n]{a})=\log \log a-\log n$ vom Numerus zum Logarithmus übergehen, wofür die oben (S. 9) beschriebene $L$-Teilung dient. Wegen des Wendens des Instruments ist dies Verfahren unbequem. Eine Erleichterung gibt Martin $\mathbf{y}^{1}$ ) an, indem er die obere schräge mm-Teilung benutzt, deren Teilungsendstriche mit denen der Stabteilung zusammenfallen. Stellt man den Läufer auf den Anfangsstrich der Stabteilung ein, so reiBt man auf der über del' mm-Teilung gleitenden Seite des Läufers auf dem Metalle einen Strich ein (bzw. bringt man einen Zeiger an), der mit $0^{\mathrm{mm}}$ zusammenfällt. Man verwendet dann bei Verschiebung des Läufers einfach die in Millimeter abgelesenen logarithmischen Längen (vgl. S. 5 Anm. 5) statt der $L$-Teilung. ${ }^{2}$ ) Die Skalenlänge von $250^{\mathrm{mm}}$ entspricht der Zahl 10, für jede Potenz von 10 ist sie um das vielfache, welches der Exponent angibt, verlängert zu denken. ${ }^{3}$ )

Um die Rechnung mit gebrochenen Exponenten noch mehr zu vereinfachen, hat Lanchester ${ }^{4}$ ) einen besonderen Läufer konstruiert, den er Radial Cursor nennt. In dem Läuferrahmen ist senkrecht zu den Skalen ein Schieber $M N O$ verstellbar, der am Ende einen Knopf $O$ trägt, um den sich ein durchsichtiger Zellhornstreifen dreht. Eine auf diesem eingeritzte gerade Linie $O J K$ geht durch die Kopfmitte. Insbesondere dient diese Vorrichtung für die Gleichung der adiabatischen Kurve $p v^{\gamma}=$ konst., so dab

1) Deutsche Mechaniker Zeitung, 1906, S. 143.

2) Beispiel: $\sqrt[\frac{7}{6}]{1,5}=(1,5)^{\frac{6}{43}}=x \quad 15 \mathrm{D} \equiv 44^{\mathrm{mm}} \quad 44 \mathrm{C}$ über $43 \mathrm{D}$. Uber $6 D$ steht $614 C$, also $44 \cdot \frac{6}{43}=6,14 \cdot 6,14^{\mathrm{mm}} \equiv 1,058 D, x=1.058$.

3) Beispiel: $234=0,0067^{x}$, also $2,34 \cdot 10^{2}=\left(6,7 \cdot 10^{-9}\right)^{x}$ $2,34 D \equiv 92,3^{\mathrm{mm}}$, also $234 \equiv 92,3+2 \cdot 250=+592,3^{\mathrm{mm}}$ $6,7 D \equiv 206,5$, also $0,0067 \equiv 206,5-3 \cdot 250=-543,5^{\mathrm{mm}}$

4) Phil. Mag. (5) 41, 52.

$$
-x=\frac{592,3}{543,5}=1,09 \text {. }
$$


z. B. $\log p_{2}-\log p_{1}=\gamma\left(\log v_{1}-\log v_{2}\right)$ ist. Schiebt man die Zunge verkehrt ein, so stehe auf Teilung $A$ : $K$ auf $p_{2}, M$ auf $p_{1}$, auf Teilung $B: J$ auf $v_{1}, N$ auf $v_{2}$ ein, dann ist auf Teilung $A$ die Strecke $M K=\log p_{2}-\log p_{1}$, auf Teilung $B$ die Strecke $N J=\log v_{1}-\log r_{2}$. Nun ist $\gamma=\frac{M K}{N J}=\frac{M O}{N O}=1+\frac{M N}{N O}$ und NO $=\frac{M N}{\gamma-1}$. Da $M N$ die Zungenbreite ist, so kann man für verschiedene Werte $\gamma$ die Länge $N^{\prime} O$ oder auch $N Z$, wo $Z$ die Mitte van $M N$ ist, berechnen und eine Skala auf $\boldsymbol{M O}$ anbringen. Reicht die Skala für die gegebenen oder gesuchten Werte von $\gamma$ nicht aus, so nimmt man statt dessen $\left.\frac{1}{\gamma} \cdot{ }^{1}\right)$

Boys') fügte bei seinem Rechenschieber noch eine Skala der Logarithmen der Logarithmen hinzu.

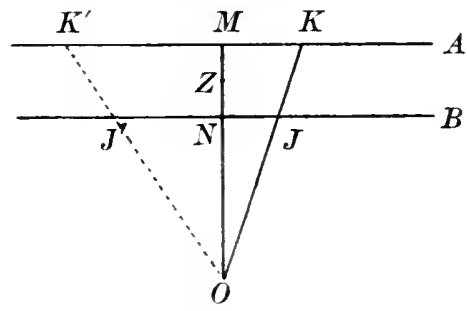

Fig. 6.

Die Prüfung des Rechenschiebers wird sich der Hauptsache nach auf das Zusammenfallen der Anfangs- und Endstriche der Teilungen auf Ober- und Unterseite der Zunge mit denen der Stabteilungen erstrecken. Auch muB das Zusammenfallen der Striche der gleich geteilten Skalen und zugleich die zur Skalenrichtung senkrechte Lage des Läuferstrichs geprüft werden. Es kommen zuweilen auch Teilungsfehler an einer einzelnen Stelle vor, die am ehesten durch Proberechnungen entdeckt werden.

Die Genauigkeit des Rechnens ist ron dem einzelnen Instrumente, insbesondere aber auch von dem Rechner abhängig. Im Mittel wird man für den Rechenschieber von $25 \mathrm{~cm}$ Länge

1) Ein anderes Beispiel ist $7^{x}=3$. Da $x$ offenbar ein Bruch ist, sucht man $\frac{1}{x}=\frac{\log i}{\log 3}$. Man stellt den Läufer in die Mitte der Skala, hält den Zellhornarm so, daB die Exponentiallinie durch $7 \mathrm{~A}$ geht und verschiebt den Knopf $O$ in der Richtung $O M$ so lange, bis der $3(A)$ auf $B$ gegenüberliegende Punkt von der Exponentiallinie getroffen wird. Dann gibt die Skalenablesung bei $Z 1.77$, also ist $x=\frac{1}{1,77}=0,565$.

2) Nature 72, 45, 1905 (8. w. unten Fürle). 
$0,2 \%$ oder $\frac{1}{500}$ Fehler annehmen können, doch geben einzelne Rechner Genauigkeiten bis zu $0,05 \%$ an.

7. Einige besondere Rechenschieber. Um eine größere Genauigkeit von etwa $0,03 \%$ zu erzielen, hat man bei gleicher Lünge des Instruments die Skalenlänge dadurch verdoppelt, daB die obere Stab- und Zungenteilung nur die Zahlen von 1 bis $\sqrt{10}=3,162$, die unteren Teilungen die Fortsetzung von $\sqrt{10}$ bis 10 enthalten. ${ }^{1}$ ) Bei dem Präzisionsschieber von Nestler ist die $L$-Teilung auf die Vorderseite genommen und ebenfalls in zwei übereinander liegende Hälften geteilt, und dadurch auf der Rückseite der Zunge Platz gewonnen, un auch den $S$ - und T-Teilungen eine doppelte Länge zu geben ${ }^{2}$ ); die Hinzufügung der gewöhnlichen Teilung von $25 \mathrm{~cm}$ Länge und zwar zweimal mit verschiedener Bezifferung ( $1-10$ und 10-100) macht den Stab nur wenig breiter und die Bildung der Quadrate und Wurzeln sehr bequem. Diesen Vorzügen steht ein geringer Nachteil gegenüber, der darin liegt, daß das Ergebnis bald auf der oberen, bald auf der unteren Skala erhalten wird. Jedoch ergibt sich als sehr einfache Regel, daß bei Benutzung der noch durch ein Kreuz ausgezeichneten Zwischenendstriche $(\sqrt{10})$ auf der Zunge, das Produkt bei der Multiplikation nicht an der anliegenden, sondern auf der gegenüberliegenden Skala abgelesen wird, und umgekehrt bei der Division, da $B$ bei Einstellungen mit Hilfe des Läufers, also Gegenüberstellung von Zahlen der oberen und unteren Skala, die Ablesung an dem einen Zwischenstrich $(\sqrt{10}$, nicht am Endstrich 1 oder 10) erfolgt. Diese Regel folgt daraus, daß die Zahlen der unteren Skala (der Fortsetzung der oberen) das $\sqrt{10}$-fache der oberen sind. Wenn also dureh Einstellung mit dem Zwischenstrich das $\sqrt{10}$-fache an der anliegenden Skala erhalten würde, so muB man

1) Bei dem Stabe von Nestler sind oben einige überschüssige Teilstriche bis 3,3, unten von 3,0 ab hinzugefügt. Der von Frank (Stuttgart) konstruierte Stab unterscheidet sich von dem oben beschriebenen von Nestler durch das Fehlen der Quadratskala und die Anbringung der $L$-Skala auf der Rückseite der Zunge. Eine Annehmlichkeit dieser Stäbe liegt in der Verteilung der Ziffern, die eine Verwechslung nahezu unmöglich macht.

2) Während der Gebrauch dieser Teilungen beim gewöhnlichen Rechenschieber sehr zurücktritt, ist hier eine 4 stellige Rechnung damit ermöglicht. 
noch mit $\sqrt{\mathbf{1 0}}$ multiplizieren oder dividieren, d. h. auf die gegenüberliegende Skala übergehen: obere Skala $\varphi(x)=\log x$, untere Skala $\chi(x)=\log (x \sqrt{10})$.

Die französische Katasterverwaltung benutzt einen Stab nach diesem Prinzip, aber von doppelten Dimensionen (Skalenlänge $1 \mathrm{~m}$, Stablänge $50 \mathrm{~cm}$ ) zur Ausführung aller einschlägigen Rechnungen. Bei langen Schiebern läBt sich jedoch die Konstanz der Übereinstimmung identischer Skalen schwerer erreichen.

Rechenschieber zu besonderen $\mathrm{Z}$ wecken sind in groBer Zahl konstruiert worden. ${ }^{1}$ ) Ein Teil bezweckt Vereinfachung und damit Verbilligung durch Weglassung ron Teilungen ${ }^{2}$ ) oder Beschränkung der Striche. ${ }^{3}$ ) Nur erwähnt seien Schieber für kaufmännische (Hannyngton) und für militärische Zwecke (Schritt$\mathrm{maB}$ ), zur Rechnung nach den tachrmetrischen Formeln (Skalen mit $\sin \alpha \cdot \cos \alpha$ und $\cos ^{2} \alpha$ ), zur Berechnung barometrischer Höhen (Sresnewsky), ${ }^{4}$ ) für Ingenieure usw. Hervorgehoben seien zwei besonders rielseitige Stäbe, bei denen durch eine praktische Anordnung der Skalen die Übersicht ermöglicht wird.

Der Nautisch-Astronomische Rechenstab ron R. Nelting ${ }^{5}$ ) gestattet das direkte Rechnen mit den Quadraten und Wurzeln der Zahlen und der trigonometrischen Funktionen und findet eine vielseitige Anwendung in der terrestrischen und astronomischen Navigation. Der $52 \mathrm{~cm}$ lange Stab trägt nicht allein auf der Ober- und Unterseite, sondern auch auf den langen Seitenflächen Teilungen, die durch einen den Stab rings umfassenden Läufer $(Z)$ mit vier Glasfenstern und Einstellstrichen in Beziehung gesetzt werden. Er enthält ferner zwei Schieberzungen ( $Y, Y_{1}$ Fig. 7, $X$ Hauptteil, $I$ Stellschrauben), die übereinander liegen, aber miteinander rertauscht werden können und deren jede auf beiden Seiten Teilungen trägt, die durch Umwenden der Zungen, sowohl

1) Favaro zählte 1879 bereits 44 Arten auf (Atti del R. Istituto Veneto di Scienze. Serie 5. T. 5, 495. Tenezia 1878/79).

2) $S$ - und $T$-Teilungen (Sickler).

3) Weglassung der letzten Unterabteilungen (Dennert u. Pape).

4) Hierzu kann auch der gewöhnliche Rechenschieber dienen, vgl. Zeitschr. f. Verm. 20, 279. 1891.

5) Hamburg $1909 \mathrm{im}$ Selbstrerlage ist unter obigem Titel eine eingehende und mit zahlreichen Beispielen erläuterte Broschüre erschienen, auf die hier verwiesen sei. (Sie enthält aber viele Druckfehler!)

Galle: Mathematiache Instrumente. 
mit der Ober-, wie mit der Unterseite des Stabes zusammengestellt werden können. Um einen Überblick zu erhalten, möge eine Zusammenstellung der Teilungen folgen ${ }^{1}$ ):

A) Obere Fläche.

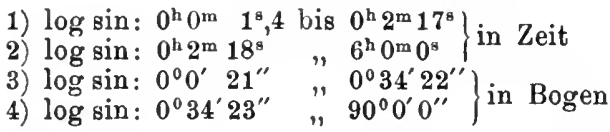

(Raum für die Zunge.)

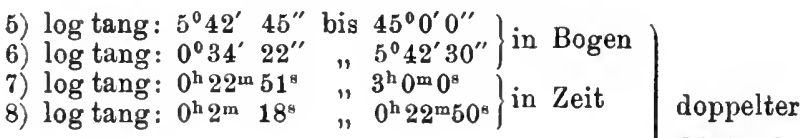

B) Vordere Seitenflächo.

$\left.\begin{array}{l}\text { 9) } \log \sin : 5^{\circ} 44^{\prime} 30^{\prime \prime} \text {, bis } 90^{\circ} 0^{\prime} 0^{\prime \prime} \\ \text { 10) } \log \sin : 0^{\circ} 34^{\prime} 23^{\prime \prime} \text { " } 5^{\circ} 44^{\prime} 15^{\prime \prime}\end{array}\right\}$ in Bogen $\quad$ 1) bis 4)

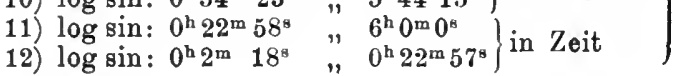

Mabstab

ron

1) Als Beispiel diene die Berechnung der Zenitdistanz $z$ eines Gestirns aus Polhöhe $\varphi$, Deklination $\delta$ und Stundenwinkel $t$. An Stelle von $\cos z=\sin \varphi \sin \delta+\cos \varphi \cos \delta \cos t$ setzt man (mit $\varphi=$ $\left.90^{\circ}-p, \delta=90^{\circ}-b\right) \sin ^{2} \frac{1}{2} z=\sin ^{2} \frac{1}{2}(b-p)+\sin p \sin b \sin ^{2} \frac{1}{2} t=$ $n^{2}+m^{2}=m^{2}\left(1+\frac{n^{2}}{m^{2}}\right)$ oder mit $\frac{m}{n}=\frac{\sqrt{\sin b \sin p \sin ^{2} \frac{1}{2} t}}{\sin \frac{1}{2}(b-p)}=\operatorname{tg} x$ erbält man $\sin \frac{1}{2} z=\frac{m}{\sin x}$. Man stellt mit dem Läufer $\frac{1}{2} t$ auf Teilung 11 oder 12 ein, addiert $\log \sin b$ und $\log \sin p$ nacheinander mit Teilung 21 oder 22 der Zunge I und liest $m$ unter dem Läuferstrich anf Teilung 14 ab. Man stellt auf Teilung 23 oder 24 bzw. bei kleinen Winkeln auf Teilung 17 oder 18 der Zunge $\frac{1}{2}(b-p)$ unter den Läuferstrich und erhält $x$ am Anfangsstrich der Zunge auf Teilung 5 oder 6 des Stabes. Man stellt $x$ auf Teilung 23 oder 24 unter den Läuferstrich und erhält $\frac{z}{2}$ auf Teilung 9 oder 10 des Stabes am Anfangsstrich bzw. Endstrich der Zunge. 
Stabteilungen.

$K \quad \frac{\lambda}{3} \log x$ von $x=1$ bis 1000

$Q \quad \frac{\lambda}{2} \log x \quad, \quad x=1 \quad, 100$

$N \quad \lambda \log x \quad, \quad x=1 \quad, \quad 10$

(Raum für die Zunge.)

L $\quad \frac{\lambda}{100} x$

W $\frac{\lambda}{100} x^{2}$

C $\frac{\lambda}{1000} y^{3}$
Zungenteilungen.

$N^{\prime}=N$

$Q^{\prime}=Q$

$Z \quad \frac{\lambda}{3}[\log \log y-\log \log \alpha]$

$=v+\frac{\lambda}{3} \log \log y$

( $\alpha$ Anfangszabl)

$W^{\prime}=W$

$L^{\prime}=I$

Von den zahlreichen durch Kombination je einer Skala des Stabes und der Zunge lösbaren Aufgaben seien einige hier angeführt.

Das Skalenpaar $K Z$ dient für die Zinseszinsrechnung: $C=\left(1+\frac{p}{100}\right)^{x}$. Sind $k_{0} z_{0}$ und $k z$ je zwei untereinanderstehende Zahlen, so ist $\log k_{0}-\log k=\log \log z-\log \log z_{0}$ also $z^{k}=z_{0}{ }^{k_{0}}$ für eine Skalenstellung konstant.

Das Skalenpaar $C L^{\prime}$ liefert die reellen Wurzeln einer kubischen Gleichung. Sind $x, y^{3}$ und $a, b^{3}$ untereinanderstehende Werte, so ist $10 x \mp 10 a=y^{3} \mp b^{3}$ für $\begin{aligned} & \text { gleich } \\ & \text { entgegen laufende Skalen. Man bringt }\end{aligned}$ die reduzierte kubische Gleichung $z^{3} \pm p z= \pm q$ durch die Substitution $z=x \sqrt{\frac{p}{10}}$ auf die Form $x^{3} \pm 10 x=10 a \pm b^{3}$, die $x=y$ entspricht.

Biquadratische Gleichungen löst das Skalenpaar $N^{\prime} K$. Für übereinanderstebende Zahlen $x, y$ und $a, b$ ist $x^{3} y=a^{3} b$, also für $x=y, y^{4}=a^{3} b$.

Das Skalenpaar $K Q^{\prime}$, also die Funktionen $\varphi(x)=\frac{\lambda}{2} \log x$, $\chi(y)=\frac{\lambda}{3} \log y$ liefern bei umgekehrter Zunge $x^{3} y^{2}=a^{3} l^{2}$, also für $x=y, x^{5}=a^{3} b^{2}$. Man verschiebt die Zunge, bis dem Werte $a$ von $Q^{\prime}$ dieselbe Zahl auf $K$ gegenübersteht, wie auf $Q^{\prime}$ dem Wert $b$ von $K$.

Die trinomische Gleichung $p x^{n} \pm q x^{n}=r$ bringt man durch die Substitution $x=\sqrt[n]{y} \sqrt[n-m]{\frac{p}{q}}$ auf die Form $y^{\frac{n}{m}} \pm y=r\left(\frac{q^{m}}{p^{n}}\right)^{\frac{1}{n-n}}$. 
Zur Berechnnng dienen $K$ und $Z$ mit den Funktionen $\varphi(x)=\frac{2}{3} \log x$, $\chi(y)=\vartheta+\frac{2}{3} \log \log y$. Man erhält $y_{1}^{a_{2}}=y_{2}^{a_{2}}$ und unter Hinzufügung der Bedingung $y_{2}-y_{1}=d, \quad y_{1}+d=y_{1}^{\frac{a_{2}}{a_{1}}}$ oder $y_{1}^{\frac{a_{2}}{a_{1}}}-y_{1}=d$.

8. Instrumente von anderer Form. Den Rechenschiebern werden ron manchen Rechnern die Rechentafeln rorgezogen, weil die oft ruckweise erfolgende Bewegung der Zunge hier wegfällt. Sie bestehen aus Skalen, die in Stücke zerschnitten und parallel zu einander in einer Ebene angeordnet sind. Eine darauf zu legende durchsichtige Scheibe mit ebensolcher Anordnung vertritt die Zunge des Rechenschiebers. Da wir sie nicht zu den eigentlichen Instrumenten rechnen können, erwähnen wir hier nur die Tafeln ron Billeter ${ }^{1}$, Klott, Ererett, Hanngngton, Scherer $\left.{ }^{2}\right)$, Proell u. a.

Werden die logarithmischen Skalen auf dem Mantel eines Zylinders angeordnet, so entstehen die sog. Rechenwalzen, wie sie Mannheim, E. Thacher ${ }^{3}$ ) und Billeter konstruiert haben. Hierher gehört auch das Instrument ron $\mathrm{Fuller}^{\mathbf{4}}$ ), dessen Teilung in einer Schraubenlinie auf einem Zylindermantel aufgetragen ist und eine Länge von $12,7 \mathrm{~m}$ hat. Die Ablesung ist $\mathrm{z}$. T. fünfstellig und die Genauigkeit etwa 1/10000. Der Zylinder läBt sich sowohl drehen als in der Richtung der Achse verschieben; die Achse wird an einem Griff gehalten, an dem ein fester Index befestigt ist. Außerdem sind zwei bewegliche Indizes vorhanden, deren Entfernung dem Abstande des Anfangs- und Endpunktes der Skala gleich ist. Bei der Multiplikation bringt man den festen Index auf einen

\footnotetext{
1) Bei seiner kleinen Tafel wird der Febler zu $\frac{1}{2500}$, bei der groben zu $\stackrel{1}{6000}$ bis 10000 angegeben.

2) Diese Tafel, die bei der Generalkommission in Kassel in Gebrauch ist, gibt vier Stellen.

3) Cylindrical Slide Rule, Abbildung bei Jordan, Handbuch der Vermessungskunde, Bd. Il, 135, 189i, 8. a. Zeitschr. f. Instrk. $\mathbf{Q 4 ,}$ 213,1904 .

4) Eine ähnliche Rechenwalze, Coordinate spirale slide rule ron Bernard, durch Stanley \& Co. in London ausgeführt, befand sich auf der Weltausstellung zu Brüssel 1910 und zeichnet sich durch Billigkeit aus. (Österreich. Zeitschr. f. Vermessungsw. 9, 47 abgebildet.)
} 
Endstrich, stellt einen beweglichen Index auf den Multiplikandus, bewegt dann den Zylinder, bis der Multiplikator den festen Index trifft, und liest das Produkt am beweglichen Index ab. Bei der Division stellt man den festen Index auf den Divisor, und den beweglichen auf den Dividendus, bewegt darauf den Zylinder, bis der feste Index mit dem Anfangspunkt der Skala zusammenfällt und erhält am beweglichen Index den Quotienten.

Die Rechenscheiben enthalten die logarithmischen Skalen in Kreisform und im allgemeinen in sich zurücklaufend. Anfänglich war nur eine Skala vorhanden und an Stelle des bei einer geradlinigen Skala notwendigen Zirkels (s. S. 3) traten zwei Zeiger. Später kombinierte man zwei oder mehrere gegeneinander verschiebbare Skalen. Boucher stellte seinen cercle à calcul in Form einer Taschenuhr her und stattete ihn mit vier konzentrischen Kreisen aus, so daß die Gesamtlänge der auf sie verteilten Skala $38 \mathrm{~cm}$ betrug. Ein fester und ein beweglicher Zeiger diente zur Einstellung. Der Rechenknecht von G. Hermann und ähnlich Sextons Omnimeter sind ebenfalls mit zwei Nadeln versehen. Es sind bei ersterem zehn konzentrische Skalen vorhanden, welche verschiedene Funktionen enthalten. ${ }^{1}$ ) Ähnlich ist auch die Rechenscheibe von Ed. Sonne von $14 \mathrm{~cm}$ Durchmesser mit drehbarem Zeiger. Erwähnt seien noch die Rechenscheiben von Clouth, Hart (Proportior mit Zeiger und Mikroskop), F. A. Meyer (Taschenschnellrechner mit einfachem Kennzifferzählwerk), A. S tein h a user (Spiralen statt Kreise), Puller, Roether, Wichmann (Haldens Calculex). ${ }^{2}$ )

Bei den Rechenrädern ist die Teilung auf der Stirnfläche der Scheibe angebracht und zwar sind zwei oder mehrere Scheiben senkrecht nebeneinander gestellt, wie bei Beyerlen's drehbarem Rechenschieber, bei dem ein Stück Gelatinepapier mit einer zur Achse parallelen roten Linie an einem festen Träger als Index oder Läufer angebracht ist. Ähnlich ist Webers Rechenkreis.

\footnotetext{
1) 1) $\alpha$ 2) $\alpha^{2}$ 3) $\alpha^{3} \quad$ 4) $2 \pi \alpha$ 5) $\frac{\pi}{4} \alpha^{2} \quad$ 6) $\left.\left.\log \alpha \quad 7\right) \arcsin \alpha \quad 8\right) \arcsin \frac{\alpha}{10}$ $9) \operatorname{arctg} 10 \alpha 10) \operatorname{arctg} \alpha$.

2) Literatur bei Lüdemann, Zeitschr. f. Verm. 36, 241, 1907, M e hmke, Numerisches Rechnen, Leipzig, 1902. Ein andres Instrument für logarithmische Rechnung kommt im folgenden unter den Differentialapparaten zur Besprechung.
} 


\section{Abschnitt.}

\section{Rechenmaschinen.}

9. Vorzüge des Rechnens mit Rechenmaschinen. Die im vorigen Abschnitt besprochenen Instrumente dienen für genäherte Rechnungen, reichen aber für viele Fälle aus, weil die zu Grunde gelegten Werte durch Beobachtung oder Messung ebenfalls nur mit genäherter Genauigkeit bekannt sind, also eine strenge Rechnung nur in formaler Beziehung ein genaues Resultat liefert. Die Ergebnisse, welche die Rechenmaschinen liefern, sind dagegen vollkommen genau. Allerdings ist diese Genauigkeit durch die Anzahl der Stellen, welche die Maschine liefert, ebenfalls begrenzt. ${ }^{1}$ ) Die Vorteile des Maschinenrechnens vor dem Gebrauche des Rechenschiebers usw. sind wesentlich folgende: 1 . beim Rechenschieber hängt die Genauigkeit von dem Zustande des Instruments $\mathrm{ab}$, insofern es immer kleinen Veränderungen durch Temperatur, Feuchtigkeit usw. ausgesetzt ist, und vom Beobachter, insbesonder von der Güte seiner Augen, während bei der Maschine, sofern sie den mechanischen Anforderungen genügt, das Ergebnis unabhängig von beiden erscheint; 2. bei den Skalenablesungen können Febler vorkommen, bei der Maschine steht das Resultat fertig da und wird bei verschiedenen neueren Ausführungen sogleich gedruckt; 3. bei den Rechenmaschinen können Kontrollen durch die Maschine ausgeführt werden. Dem gegenüber stehen als Nachteile die gröBeren Anschaffungskosten und in den meisten Fällen der Praxis die überflüssige Kraftanstrengung infolge Mitführung einer mehr oder weniger großen Anzahl unnötiger Ziffern.

10. Zählwerk. Jede Rechenmaschine enthält ein Zählwerk, das meistens aus einer Reihe horizontal ${ }^{2}$ ) liegender, kreisförmiger Scheiben besteht, auf deren jeder die Ziffern $0,1,2, \ldots 9$ in gleichen Abständen am Rande aufgetragen sind. Jede Scheibe ist um ihre senkrechte Achse drehbar und mit einer feststehenden Platte bedeckt, die ein Schauloch oder Fenster enthält, durch das immer nur eine der Ziffern sichtbar ist. Folgen die Ziffern ein-

1) Nur bei einer Maschine (GauB) ist die Stellenzabl des Multiplikators oder Quotienten unbegrenzt, aber dies ist nur bei der Division von Vorteil.

2) Bei manchen Maschinen (s. u. Odhner) stehen die Scheiben vertikal. 
ander im entgegengesetzten Sinne wie diejenigen des Zifferblattes einer Uhr, so werden bei Drehung der Scheibe im Sinne des Uhrzeigers nacheinander die Zahlen, in der richtigen Reihenfolge zunehmend, in das Schauloch gelangen, während bei entgegengesetzter Drehung die in dem Schauloche erscheinenden Ziffern in abnehmender Reibe auftreten. Durch Drehung einer solchen Scheibe um eine gegebene Zahl (3) von Zifferintervallen kann man daher zu einer im Schauloch gerade sichtbaren Zahl (5) jene Zahl (3) addieren oder von ihr subtrahieren, und man erblickt im Schauloch nach der Drehung die Summe (8), falls diese nicht 9 überschreitet, oder bei entgegengesetzter Drehung die Differenz (2), sofern sie 0 oder größer als 0 ist.

11. Zehnerübertragung. Wenn man aber bei einer solchen Reihe von Scheiben, deren Schaulöcher nebeneinander liegen, z. B. in dem am weitesten rechts gelegenen Fenster die Zahl 5 erblickt und durch Drehung dieser Scheibe im Uhrzeigersinne um 9 Stellen die Ziffer 4 in diesem Schauloch erhält, so muB man, um das richtige Resultat $14 \mathrm{zu}$ erhalten, die links daneben stehende Scheibe, wenn in ihrem Schauloch 0 stand, um eine Stelle drehen, so daB 1 darin erscheint. Um dies nicht mit der Hand ausführen zu müssen, ist eine Übertragungsvorrichtung nötig, die darin bestehen kann, daB die Achse jeder Scheibe ein Zahnrad mit zehn Zähnen trägt, die den zehn Ziffern der Scheibe entsprechen und daß der Zahn, welcher der Null entspricht, genügend lang ist, um in das Nachbarrad einzugreifen und dies dadurch um einen Zahn weiter zu drehen. Diese Vorrichtung würde für das Rad am rechten Ende, dessen Scheibe die Einer anzeigt, genügen, da es ja nur das Rad der Zehnerscheibe neben sich hat. Bei den andern Scheiben würde aber ebenso ein Eingriff des verlängerten Zahnes in das rechts gelegene Zahnrad erfolgen, während doch nur eine Übertragung nach links beabsichtigt ist. Es gibt daher, je nach der Ausführung der Maschine, verschiedene Arten ${ }^{1}$ ) dieser Zehnerübertragung und von der Zuverlässigkeit des Übertragungsmechanismus hängt wesentlich die Güte der Maschine ab.

1) Wir besprechen sie an anderer Stelle. Das von Pascal erfundene Prinzip, das wir hier angegeben haben, ist bei den einfachen Additionsmaschinen (Goldmans Contostyle, Bollée Compteur) anwendbar, bei denen z. B. auf parallel geführten Metallstreifen, die über Rollen in sich zurücklaufend geführt sind, die Zahlen stehen und nach links eine Übertragung angebracht werden kann. 
Die Drehung der Scheiben erfolgte bei der ersten Maschine, die Pascal 1642 konstruierte, mit der Hand, bei andern Maschinen durch Niederdrücken von Tasten, indem entweder jeder Ziffer von 1 bis 9 eine Taste entsprach, oder aber für die Einer, Zehner, Hunderter usw. je neun Tasten in parallelen Reihen angeordnet wurden. ${ }^{1}$ )

12. Schaltwerk. Um mit diesen Maschinen zu multiplizieren (dividieren), ist eine wiederholte Addition (Subtraktion) erforderlich. Hierbei ist es zu mühsam, nacheinander jede einzelne Scheibe um die geforderte Stellenzahl zu drehen, man benutzt daher ein Schaltwerk. Die älteste, aber heute noch gebräuchliche Ausführung eines solchen verdanken wir Leibniz (1673), der die Staffelwalze und damit die erste zur Multiplikation brauchbare Maschine erfand. ${ }^{2}$ ) Ein solches Schaltwerk dient dazu, gleichzeitig sämtliche Scheiben und zwar jede um die geforderte Stellenzahl weiter zu drehen.

Wenn wir uns eine Anzahl von zehn völlig gleichen Zahnrädern zentrisch so aufeinander gelegt denken, daB ihre Zähne und Zahnlücken sich decken, so bilden sie zusammen einen Zylinder mit Längsleisten (Fig. 9). Wir stellen uns nun weiter vor, daB das erste Rad vollständig ist, bei jedem folgenden aber, der Reihe nach, ein Zahn mehr fortgeschnitten wird, so daB das vorletzte Zahnrad nur einen, das letzte keinen Zahn besitzt. Dann haben auf dem Zylinder, den die Zahnräder bilden, die Leisten verschiedene Längen, die der Reihe nach über $9, \delta, 7, \ldots 1$ Zahnräder reichen. Ein solcher, in Wirklichkeit aus einem Stück hergestellter Zylinder, wird nach Reuleaux Staffelwalze genannt.

Wird nun auf einer der Walzenachse parallelen vierkantigen ${ }^{3}$ ) Achse (Fig. 8) ein Zahnrad $Z$ mit zehn gleiclimäBig verteilten Zähnen verschoben, so wird es, wenn es dem ersten der Räder, aus deuen wir uns die Staffelwalze zusammengesetzt dachten, gegenïbersteht und zum Eingriff kommt, bei einer vollen Umdrelung der Walzenachse um neun Zähne weitergedreht. Bei der weiteren Verschie-

1) Additionsmaschine ron Max Mayer, konstruiert ron A. Barthelmes, 1887. Auch verband man damit eine Vorrichtung zum Drucken der Summanden und der Summen: Burrough.

2) Die nach seinen Angaben gebaute Maschine konnte allerdings nicht in Gang gebracht werden, vgl. Zeitschr. f. Term., 26, 292, 1897.

3) Längs einer solchen Achse kann ein lose aufsitzendes Rad mit viereckigem Ansschnitt verschoben werden und dreht sich doch bei einer Drehung der Achse mit. 
bung erleidet $Z$ Eingriffe von acht Staffelleisten bei einer Umdrehung der Walzenachse usf. Am andern Ende angelangt, wird $Z$ nur um einen Zahn weiter gedreht und nach der letzten Verschiebung steht es dem glatten Zylinder gegenüber und wird gar nicht gedreht.

In dem Gehäuse, wo diese Einrichtung angebracht ist, sei nun im oberen Deckel ein Schlitz parallel den Achsen der Staffelwalze und des verschiebbaren Zahnrades $Z$ angebracht und in ihm ein Stab mit einem Knopf, der den Stab am Hineinfallen hindert, senkrecht aufgehängt (Fig. 8). Mit Hilfe dieses Stabes wird $Z$ auf seiner Achse verschoben. Am Rande des Schlitzes sind den verschiedenen Stellungen von $Z$ entsprechende Ziffern $9,8, \ldots 1,0$ angeschrieben und der Knopf ist mit einem Zeiger versehen. Stellen wir den Zeiger auf eine bestimmte Ziffer ein, so wird bei einer durch eine Kurbel bewirkten Umdrehung der Staffelwalze das Zahnrad um dieselbe Anzahl von Stellen gedreht, wie die Ziffer angibt. Durch ein Kegelgetriebe ${ }^{1}$ ) überträgt das $\operatorname{Rad} Z$, bzw. seine sich mit ihm drehende Achse $c$ (Fig. 8) die Drehung auf eine unter dem Deckel des Gehäuses, in dem sich auch der Schlitz befindet, horizontal gelagerte Ziffernscheibe. Man erblickt dann durch das im Deckel freigelassene Schauloch die betreffende Ziffer.

Nun ist, wie schon erwähnt, nicht ein Schlitz, sondern eine Anzahl paralleler Schlitze vorhanden und unterhalb eines jeden befindet sich eine ebensolche Einrichtung, wie unter dem ersten. Ferner ist durch eine Kurbel $b$ (Fig. 8) mit einer langen, quer zu den vorgenannten Achsen liegenden Achse $a$ (Fig. 8) die Möglichkeit gegeben, durch eine einzige Kurbeldrehung mittels Kegelräderïbertragung sämtliche Staffelachsen gleichzeitig einmal herumzudrehen. ${ }^{2}$ )

1) Zahnräder, bei denen die Zähne auf dem kegelförmig abgeschrägten Radkranze liegen, können ineinander eingreifen, wenn ihre Ebenen schräg oder sogar senkrecht zueinander liegen (z. B. die beiden Räder in Fig. 8 vorn links), während gewöhnliche Zahnräder nur zum Eingriff gelangen können, wenn sie in derselben Ebene liegen.

2) Würde z. B. in den vier am weitesten rechts gelegenen Schlitzen die Zahlen 9876 eingestellt, während die übrigen Knöpfe auf 0 stehen, so wird eine Multiplikation von 9876 mit 3 durch dreimalige Kurbeldrehung erreicht. Bei der ersten Umdrehung erscheint die Zahl 9876 selbst in den vier rechts liegenden Schaulöchern. Bei der zweiten Umdrehung erhält man 19752, bei der dritten 29628, wenn Zehnerübertragung stattfindet. Soll dagegen zu 9876 eine andere 
Um auch subtrahielen und damit dividieren zu können, ist ein Umschalter vorgesehen. Wir haben ein Kegelrad an der Achse des Rades $Z$ erwähnt, welches die Drehung dieser Achse auf die senkrechte Achse der Zählscheibe überträgt. Nun sind zwei solche Kegelräder auf der Achse von $Z$ angebracht, die von entgegengesetzten Seiten in das Zählscheibenrad eingreifen und es demgemäB in verschiedener Richtung drehen können. Durch einen Stellhebel $d$ und $e$ (Fig. 8) kann nun gleichzeitig für sämtliche Achsen aller Räder $Z$ die Verschiebung der Kegelräderpaare bewerkstelligt werden, so daB dis Zahlen auf allen Zählscheiben entweder gleichzeitig zu- oder

abnebmen, wenn man die Kurbel dreht.

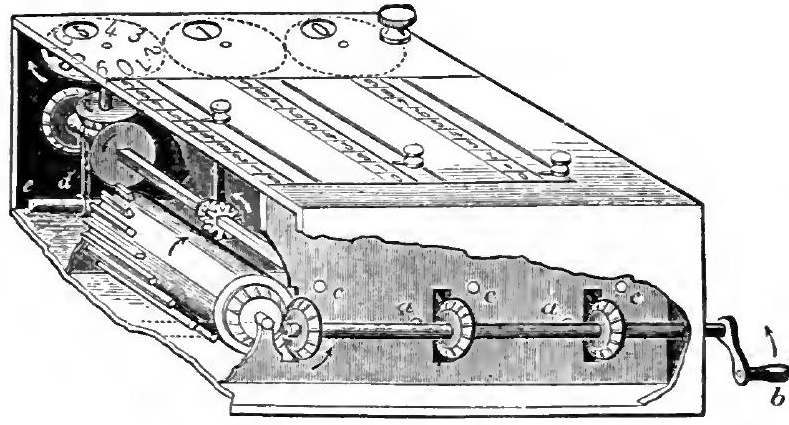

Fig. 8 .

Es muB noch erwähnt werden, daB die Verteilung der zwar unter sich gleich weit abstehenden Zahnleisten der Staffelwalzen keine gleichförmige über den Umfang der Walzen ist. Vielmehr nehmen die Leisten nur einen Teil des Zylinderumfanges ein, so z. B. bei Burkhardt ${ }_{25}$, während ${ }^{16} / 25$ frei bleiben, bei Thomas $9 / 22$ gegen $13 / 23$. Dies hängt mit der Zehnerübertragung zusammen, dereu Einrichtung wir jetzt leicht erörtern können. Die Achsen der Zahnräder $Z$ tragen nämlich in ihrer Verlängerung noch andere Räder $Y^{1}$ ), die nicht mit den Zahnleisten der Walzen in Berührung kommen. Jede Walzenachse trägt auch noch in ihrer Verlängerung einen Zahn $X$ (Fig. 9), der aber für gewöhnlich nicht in das zugehörige $\operatorname{Rad} Y$ eingreift. Wenn aber eine der $\mathrm{Z} \ddot{\text { ähl }}$ scheiben $S_{i}$ von 9 auf 0 übergeht, so verschiebt ein unten an ihr

Zahl 11325 addiert werden, so dreht man zunächst einmal und erbält 9876 in den Schaulöchern, dann stellt man die Knöpfe auf 11325 in den fünf rechts liegenden Schlitzen und erhält bei nochmaliger Kurbelung 21201 in den Schaulöchern.

1) In Fig. 8 nicht sichtbar. 
befindlicher Daumenansatz mittels einer Hebelübersetzung den Zahn $X_{i+1}$ der links nächstfolgendeu Walze in die Ebene des zugehörigen Rades $Y_{i+1}$, so daß dieses um eine Stelle weiter gedreht wird und die Zahl auf der zugehörigen Zählscheibe $S_{i+1}$ sich um 1 ändert. Dieser Eingriff des Zahnes $X_{i+1}$ in das $\operatorname{Rad} Y_{i+1}$ darf aber nicht gleichzeitig mit dem Eingriff einer Zahnleiste der Walze $W_{i+1}$ in das $\operatorname{Rad} Z_{i+1}$ erfolgen. Denn da $Y_{i+1}$ und $Z_{i+1}$ auf derselben Achse sitzen, so würde die gleichzeitige Wirkung den einen von

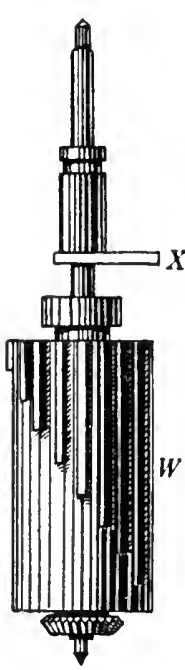

Fig. 9 . beiden Eingriffen überflüssig machen oder bei nicht vollst ändiger Gleichzeitigkeit eine Störung verursachen. Deshalb darf der Eingriff des Zabnes $X_{i+1}$ erst erfolgen, wenn bereits alle Zahnleisten der Walze $W_{i+1}$ ihre Wirkung auf das Zahnrad $Z_{i+1}$ ausgeübt haben. Hieraus ist erklärlich, weshalb die Zahnleisten noch einen Teil des Walzenzylinders frei lassen müssen, aber dieser freie Teil brauchte nicht so bedeutend zu sein, wenn nur die eine Walze $W_{i+1}^{r}$ in Betracht käme. Der Zahn $X_{i+1}$ darf aber auch erst wirken, wenn sämtliche Zahnleisten der (rechts) vorangehenden Walze $W_{i}$ einschließlich des an ihr befindlichen Zahnes $X_{i}$ ihre Rolle fertig gespielt haben. Denn es kann ja gerade der Zahn $X_{i}$ sein, welcher die Scheibe $S_{i}$ von 9 auf 0 bringt und demnach erst die Zehnerübertragung auf die Scheibe $S_{i+1}$ nötig macht. Aus diesem Grunde darf die Stellung der Zahnleisten auf den verschiedenen Walzen nicht dieselbe sein; die Walzen, die ja an sich ganz gleich sind, müssen sozusagen, gegen einander verdreht sein. Es ist dies auch noch deshalb nötig, weil beim gleichzeitigen Eingreifen der Staffelwalzen eine große Kraft zum Drehen der Kurbel erforderlich wäre und außerdem starke Spannungen auftreten würden.

13. Einteilung der Rechenmaschinen. Diejenigen Maschinen, die zwar eine Zehnerübertragung, aber kein Schaltwerk besitzen, werden als Additionsmaschinen bezeichnet, wie Roths Additioneur, Webbs Adder und zahlreiche in kaufmännischen Betrieben verwendete Abarten. Bei ihnen finden sich häufig selbsttätige Vorrichtungen zum Drucken der addierten Zahlen und ihrer Summen auf einem weiterlaufonden Papierstreifen, wie bei Burroughs Registering Accountant. Tritt ein Schaltwerk hinzu, so erhält man eine erweiterte Additionsmaschine. 
Um diese aber zum Multiplizieren auch mit mebrstelligen Multiplikatoren benutzen zu können, muB beim Übergang von einer Stelle des Multiplikators zur nächsten (von den Einern zu den Zehnern, Zehnern zu den Hunderten usf.) das Zählwerk gegen das Schaltwerk jedesmal um eine Stelle rerlegt werden. Diese Verrückung findet entweder durch Umbeben mit der Hand von einem Einsatz zum andern statt, wodurch bei der beschriebenen Einrichtung jede Staffelwalze mit dem nächstfolgenden Zahnrad der ihm entsprechenden Zählscheibe in Eingriff kommt, oder die Verschiebung findet auf Schienen statt und wird durch Anschläge begrenzt und durch Druck auf einen Knopf ausgelöst oder endlich sie erfolgt selbsttätig nacb jeder Kurbeldrehung, wobei dann durch einen besonderen Ausschalter die Möglicbkeit gegeben sein muB, sio auBer Tätigkeit zu setzen (im Falle der Addition). Eigentliche $\mathrm{Mul}$ = tiplikationsmaschinen sind mit einem Multiplikationskörper versehen, wie er zuerst von Bollée (1888) angewandt worden ist. Hierzu kommen als vierte Art noch die Differenzenmaschinen.

14. Thomas-Burkhardtsche Maschine. Die erste, wirklich brauchbare Maschine, die mit der Leibnizschen Ähnlichkeit hatte, war 1776 von dem Pfarrer Mathias $\mathrm{Hahn}$ in Echterdingen ge- baut worden. Dasselbe Prinzip lag auch derjenigen des Ingenieurhauptmanns J. H. Müller in Gieben (1783) zugrunde, zur allgemeinen Verbreitung kam aber erst der Arithmometer des Pariser Versicherungsdirektors Thomas aus Colmar (1821), den dann Burkhardt in Glashütte (1878) weiter verbessert hat. Hierau schlieBen sich dann zahlreiche neuere Maschinen an, die unter den Namen Arithmometer, Archimedes, PeerleB, Saxonia, Tim, Unitas, Bunzel-Delton, Austria, Ideal usf. verbreitet sind. Die Beschreibungen, die im Voranstehenden gegeben sind, beziehen sich auf die Burkhardtsche Konstruktion, im folgenden sollen für die hauptsächlichsten Typen die wesentlichen Abweichungen und Ergänzungen folgen. Eine Anzahl teils gemeinsamer, teils besonderer Einrichtungen muB hierbei übergangen werden. Es sei nur erwähnt, daB an Stelle der Zehnerübertragung bei der letzten Stelle, wo sie aufhört, ein Glockensignal als Warnungszeichen eingeführt ist, daß ferner Löschrorrichtungen alle Zühlscheiben gleichzeitig auf die Einstellung ,Null" zurückfülıren, daB bei manchen Masclinen zwei Reihen von Zählscheiben vorhanden sind, von denen die eine das Zwischeuresultat festhält, während eine neue Operation ausgeführt wird und dergl. mehr. 
15. Divisionsmaschine Mercedes-Euklid. An diese Rechenmaschinen schlieBt sich in der Form die von Ch. Hamann (Friedenau bei Berlin),konstruierte und Mercedes-Euklid benannte an (Fig.11). Bei ihr sind die Staffelwalzen durch 10 Zahnleisten $Z$ ersetzt, welche in ihrer Längsrichtung verschiebbar quer unter den Einstellschlitzen angebracht sind. Ihre Verschiebung wird durch einen sogenannten Proportionalhebel bewirkt, mit dem"sie durch Gelenke verbunden sind. Dieser Hebel $H$ wird um einen seiner Endpunkte durch eine Pleuelstange $P$, die ihn in der Mitte anfaßt, bei einer Um-

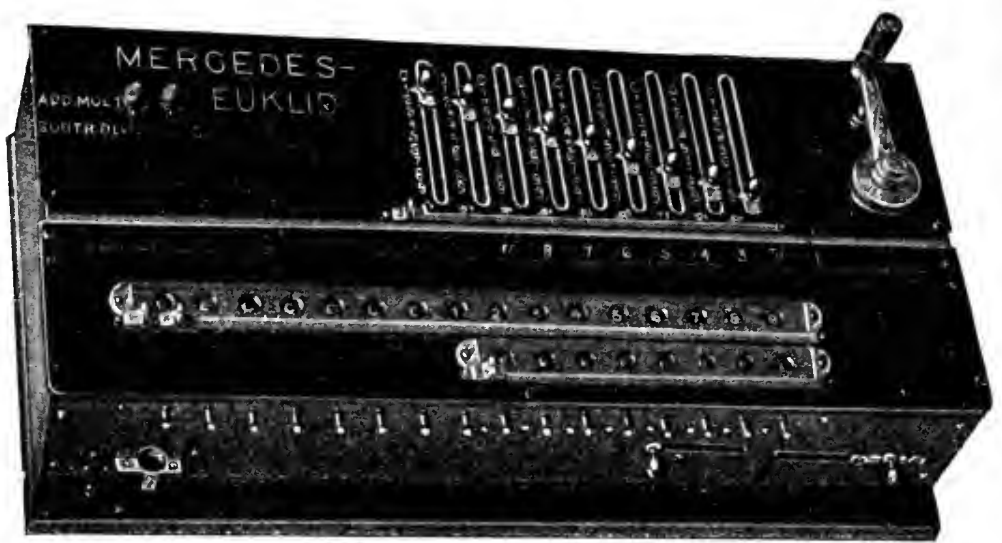

Fig. 10.

drehung der Kurbel $K$ gedreht. Jeder Gelenkpunkt und damit jede Zahnleiste $Z$ verschiebt sich dadurch um eine Strecke, die den an den Schlitzen angeschriebenen, darüber stehenden Zahlen proportional ist. Stellt man nun mit den Einstellknöpfen über die Zahnleisten Zahnräder, welche unter den Knöpfen angebracht sind, so greifen diese in die Zahnleisten ein und werden um so viel Stellen gedreht, als sich die betreffenden Zahnleisten verschieben, also um die Zahl, die den Einstellungen der Knöpfe gleich ist. Durch einen Umstellhebel kann aber der Proportionalhebel zur Drehung um seinen anderen Endpunkt eingestellt werden. Dann beschreiben die Gelenkpunkte und die Zahnstangen, die vorher $0,1,2,3, \ldots 9$ Einheiten bei einer Kurbelumdrehung zurücklegten, 9, 8, 7, . 0 Einheiten, d. h. die Érgänzungen der an den Schlitzskalen angezeigten Zahlen zu 9. Diese Einstellung dient daher zur Subtraktion, die in der Tat durch Addition der 
dekadischen Ergänzungen ausgeführt werden kann. Wenn noch die Einerstelle um eine Einheit erhöht und die höchste Stelle um eine Einheit erniedrigt wird ${ }^{1}$ ), was durch besondere Vorrichtungen der Maschine besorgt wird, so erhält man das richtige Resultat. Für die Multiplikation (Multiplikator) und Division (Quotient) ist es notwendig, daB auch die Kurbelumdrehungen gezählt werden und diese Zahlen in besonderen Schaulöchern (die den Stellen der Einer, Zehner usf. entsprechen) sichtbar werden, wie dies auch bei den anderen Maschinen der Fall ist. Nun ist aber bei dieser Maschine auch für das Umdrehungszählwerk die Zehnerübertragung vorhanden und eine Umschaltung von Addition auf Subtraktion vorgesehen. Hierdurch ist die Möglichkeit einer völlig automatischen

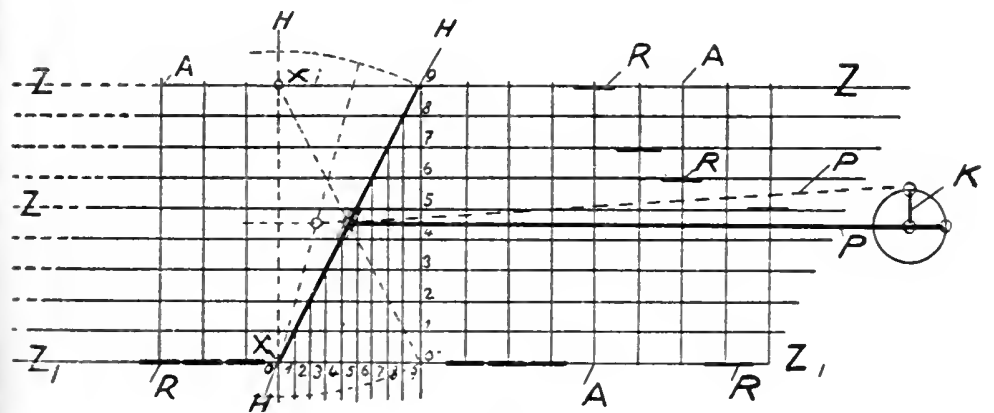

Fig. 11.

Division gegeben, wobei durch sehr bequeme Anordnung der beiden Umschalter für das Schaltwerk (Proportionalhebel) und die Kurbelumdrehungen die jedesmalige Einstellung beider durch ei n e $n$ Handgriff erledigt wird (Fig. 10 links oben). Auch wird bei jeder Umschaltung des Proportionalhebels, wenn nicht eine besondere Hebelvorrichtung dies verhindert ${ }^{2}$ ), das Zählwerk selbsttätig um eine Stelle nach links verrückt. Ferner tritt an die Stelle der bei der letzten Ziffer nicht mehr vorhandenen Zehnerübertragung nicht ein Glockenzeichen, sondern eine mit Federdruck bewirkte Sperrung der Kurbel.

\footnotetext{
1) Beispiel : $384923-97605=384923+(99999-97605)-100000$ $+1=384923+2394-100000+1$.

2) Bei der Addition und Subtraktion darf keine Verrückung des Schaltwerkes gegen das Zählwerk stattfinden, wie schon oben erwähnt wurde.
} 
Die automatische Division, durch die sich diese Maschine auszeichnet, beruht auf folgendem Vorgang, bei dem wir uns auf 2 Stellen des Quotienten $c$ und $d$ beschränken: Es sei

also

$$
\frac{a}{b}=c \cdot 10^{n}+\frac{r_{c}}{b}, \quad \frac{r_{c}}{b}=d \cdot 10^{n-1}+\frac{r_{d}}{b},
$$

$$
\frac{a}{b}=c 10^{n}+d 10^{n-1}+\frac{r_{d}}{b},
$$

dann führt man zunächst $c+1$ Kurbeldrehungen (Kurbelzählwerk auf Addition) bei Stellung des Proportionalhebels auf Subtraktion aus und erbält

$$
\frac{a}{b}=(c+1) 10^{n}+\frac{r_{c}-b \cdot 10^{n}}{b} .
$$

An einer Fortsetzung der Kurbeldrehung hindert die Sperrung der Kurbel, sobald $r_{c}-b \cdot 10^{n}$ negativ ist. Jetzt schaltet man den Hebel $H$ auf Addition und das Kurbelzählwerk auf Subtraktion und erhält bis zur Sperrung der Kurbel

$$
\frac{r_{c}-b \cdot 10^{n}}{b}=-(10-d) 10^{n-1}+\frac{r_{d}}{b} .
$$

Beide Operationen zusammen geben daher denselben Ausdruck für $\frac{a}{b}$ wie oben. ${ }^{1}$ )

1) Beispiel: $a=225, b=7, c=3, d=2, r_{c}=15, r_{d}=1,10^{n}=10$, $10^{n-1}=1 . \quad(a: b=32$, Rest 1.) Wir nehmen eine 9 stellige Maschine an und stellen mit den Wirteln an der Vorderfläche der Maschine bei äuBerster Verschiebung des Wagens nach rechts in der Reihe der 16 Schaulöcher des Dividendus, von links beginnend, 225 ein. Als Divisor stellen wir 7 (da wir dies in diesem Beispiel übersehen, in dem zweiten der Schlitze) ein. Bei Stellung des Proportionalhebels (linker Knopf Fig. 10 oben links) auf Subtraktion wird die dekadische Ergänzung addiert, also bei jeder Kurbeldrehung ...930. Dies gibt, bei der ersten Kurbeldrehung zu 225 addiert, 155 . Wir drehen die Kurbel weiter und erhalten der Reihe nach $085,015,945$. Bei diesem Übergang mïßten die Zahlen 9 nach links fortgesetzt werden (da die dekadische Ergänzung ebenfalls mit unendlich vielen Zahlen 9 nach links zu denken ist). Die letzte Stelle links überträgt sich aber nicht mehr, sondern löst statt dessen die Hemmung aus, so daB eine weitere Kurbeldrehung unmöglich ist. Das Resultat ist bis hierher $945-1000$ $=-55=r_{c}-b \cdot 10^{n}$. Das Kurbelzählwerk (vordere Reihe mit 8 Schaulöchern für den Qnotienten) zeigt 4 positive Drehungen in der ersten Stelle an.

Stellt man jetzt den Proportionalhebel auf Addition und gleich- 
16. Odhners Arithmometer. Die nach dem System von dem'schwedischen Ingenieur W. Th. Odhner in Petersburg (1878) konstruierten Maschinen (Odhner, Brunsviga 1892, Brunsvigula, Merkur, Arithmotyp-Trinks, Berolina, Triumphator, Monopol) haben an Stelle der Staffelwalzen Zahnräder $\boldsymbol{E}$ mit einziehbaren Zähnen. ${ }^{1}$ ) Bei der Einstellung der Stellhebel (die an Stelle der Knöpfe [s. S. 26] treten) in den auf einer zylinderförmig gewölbten Fläche angebrachten Scblitzen werden in den Zahnrädern $E$ so viele Zähne vorge-

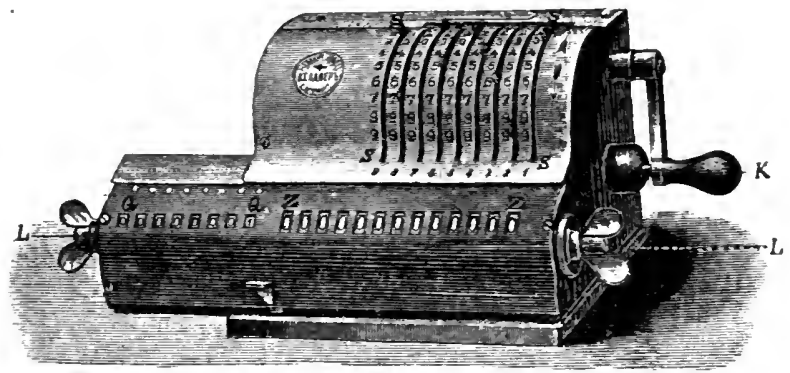

Fig. 12.

S S Schlitze, ZZ Produkt (Dividendus), $Q Q$ Multiplikator (Quotient), $L L$ Lobschvorrichtang, $K \mathrm{Karbel}$.

schoben, daB die am Schlitz eingestellte Zahl ron Zähnen in Wirksamkeit tritt (Fig. 13). Das Zahnrad Z (s. S. 25) verschiebt sich hier nicht, sondern bleibt immer seinem besonderen Rade $E$ gegenüber. Auf diese Weise wird auch weniger Raum beansprucht, da auch diз Zählscheiben auf einer gemeinsamen horizontalen Welle neben-

zeitig das Kurbelzählwerk (reehter Knopf Fig. 10 oben links) auf Subtraktion, so wird gleichzeitig das Zählwerk um eine Stelle nach links verrückt, so daB 7 über der letzten Ziffer von 945 steht. Macht man jetzt $10-d=8$ Kurbeldreliungen, so erhält man $952,959,966$, $973,980,987,994,001$. Jetzt löst der Übergang ron 9 auf 0 in der letzten Stelle links die Hemmung und hindert eine weitere Drehung. (Wenn wir nicht mit dem Reste $r_{d}=1$ aufhören wollen, mïssen wir wieder den linken Knopf auf Subtraktion, den rechten auf Addition stellen, und man kann beliebig weit fortfahren, wenn man am Ende angelangt, den letzten Rest wieder nach links in der Dividendenreihe überträgt.) Das Kurbelzählwerk zeigt in der zweiten Stello s negative Umdrehungen an, oder da bei ihm anch Zehnerïbertragung stattfindet, 80 liest man im ganzen $40-8=32$ ab.

1) Dieser Gedanke rührt auch ron Leibniz her und war vielleicht bei seiner zweiten (verschollewen) Maschiue verwirklicht. Vyl. Mehmke, Numerisches Rechnen S. 965. 
einander angebracht werden konnten. Die Zahlen (ZZ Fig. 12) stehen auf ihren Stirnflächen dichter nebeneinander als bei anderen Maschinen mit horizontal liegenden Rädern. Ein Vorteil dieser Maschinen vor allen anderen ist die Bewegung der Kurbel in einer senkrechten Ebene, wobei der Arm weniger ermüdet, als bei horizontaler Drehung. Dieselbe Schaltwerkeinrichtung haben die Maschine von Otto Büttner und die Duplex-Rechenmaschine von Küttner,

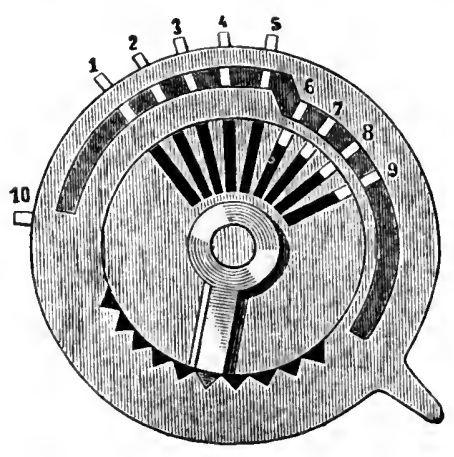

Fig. 13 . letztere auch mit Odhners Anordnung der Zählscheiben. Die anderen Einriclitungen sind $\mathrm{z}$. 'T. verschieden, z. B. hat Büttner keine Umschaltung für Addition und Subtraktion, sondern die Kurbel wird für letzteren Zweck rückwärts gedreht, ferner stehen im Stellwerk die Ziffern in gerader Reihe nebeneinander, was übrigens bei den meisten neueren Maschinen aller Konstruktionen durch Sichtbarmachung der Einstellung in einer besonderen Reihe von Schaulöchern ebenfalls herbeigeführt wird.

17. Rechenmaschine Gauß. Wie die Hahnsche und J.H. Müllersche Rechenmaschine haben die Circular CalculatingMachine von J. Edmonson in Halifax (Engl.) und die deutsche „GauB" von $\mathrm{Ch}$. Hamann eine kreisförmige Gestalt (Fig. 14). Die Vorzüge der GauB bestehen in ihrer kleinen Form und entsprechend geringerem Preise und ihrer Handlichkeit, in dem durch die noch zu erwähnende Stufenscheibe erzielten sanften Gange und in dem durch ihre Bauart begründeten Umstande, daß eine Einschränkung der Ziffernzahl des Multiplikators und des Quotienten nicht eintritt, wenn die Maschine zunächst auch auf 10 Stellen eingerichtet ist. Diesen Vorteilen steht ein wesentlicher Nachteil gegenüber, der darin liegt, daB die eingestellten und die abzulesenden Ziffern im Kreise, also entweder für den Beschauer zum Teil auf dem Kopfe stehen, oder teils ihre Köpfe nach innen und teils nach außen richten.

Die Maschine besteht aus einer festen mit dem FuB verbundenen Dose und der durch einen Hebel abhebbaren Kapsel, in deren Deckel sich in radialer Anordnung 6 Einstellschlitze befinden. (Fig. 14.) Unter jedem dieser Schlitze liegt eine ebenfalls radiale Achse $f$ mit einem darauf verschiebbaren Zalınrad $z$, das durch 
eine an jedem Einstellknopf $S$ unten angebrachte Gabel auf $f$ verstellt wird (Fig. 15). Es sei sogleich noch erwähnt, daß am äuBeren Ende jeder Achse $f$ auch noch ein festes Zahnrad $r$ mit 10 Zähnen sitzt, das sich mit seiner Achse dreht.

Über dem Deckel befindet sich die Kurbel $K$, welche eine durch seine Mitte und durch die Maschine hindurchgehende senkrechte Achse $x$ drelit. Diese Acbse trägt in der Mitte die horizontal liegende Stufenscheibe Sch. Sie dreht sich mit der Kurbel, während die anderen Teile der Maschime in ihrer Lage bleiben. Die Oberseite dieser Scheibe trägt 9

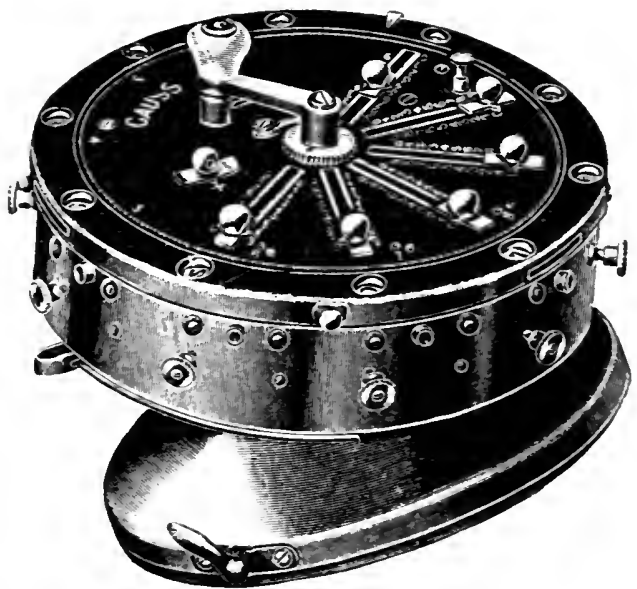

Fig. 11. konzentrisch angeordnete Reihen ron Zähnen, von denen die innerste 9 Zähne, jede weiter nach auBen liegende einen Zahn weniger be-

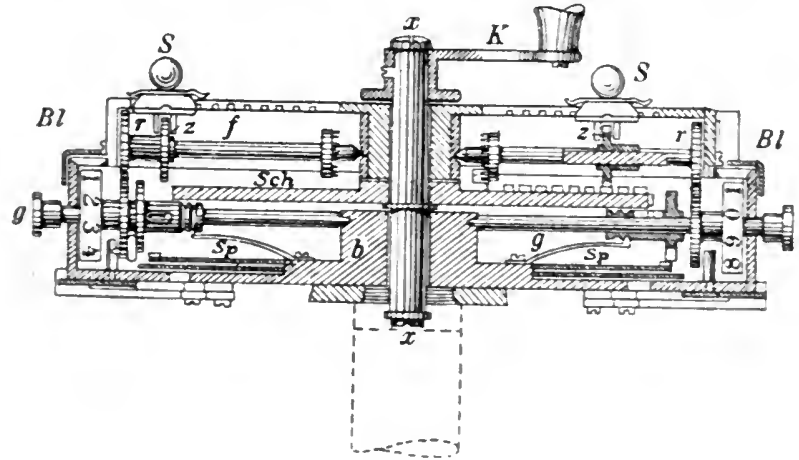

Fig. 15.

sitzt, bis zur äuBersten mit nur einem Zahne (Fig. 16). Wenn ein Knopf an einem der Einstellschlitze des Deckels auf eine bestimmte Zahl der am Schlitz stebenden Skala eingestellt wird, so gebt das 
Zahnrad bei der Drehung der Scheibe über die Reihe mit derselben Zahl von Zähnen und erfährt daher eine Drehung um diese Zahl. Die Zahnringe nehmen nur einen Bruchteil des ganzen Umkreises der Scheibe ein. Dicht hinter ihnen folgt bei der Drehung

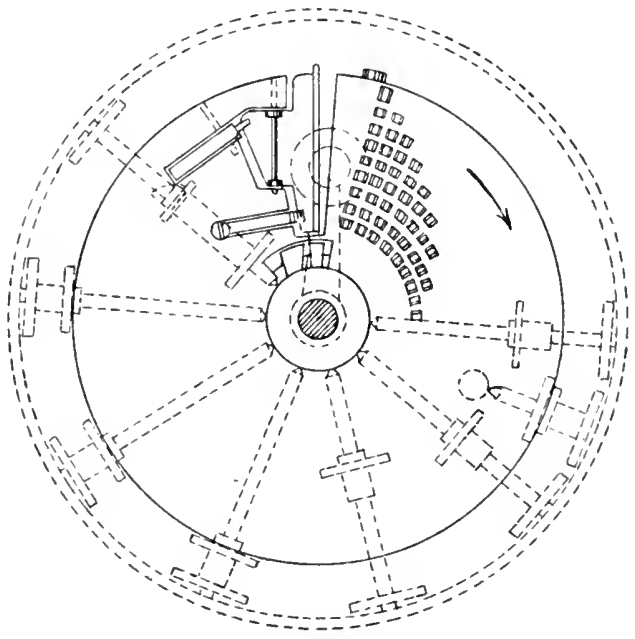

Fig. 16. der Scheibe ein zungenartiges Sperrstück. Auf jeder Achse $f$ befindet sich nämlich am zentralen Ende eine Scheibe mit 10 nach dem Innern der Maschine gerichteten Stiftchen. Wenn die Achse $f$ über sämtliche Zähne hinweggegangen ist und das Rädchen $z$ die vorgeschriebene Drehung vollführt hat, so schiebt sich das Sperrstück zwischen die Stiftchen und hält nun die Achse fest, damit sie sich nicht weiter drehen kann.

Unterhalb der Scheibe liegt das Zählwerk, das sich also in der eigentlichen Dose befindet. Indem die Stufenscheibe nicht bis an den Umfang der Dose heranreicht, übertragen die am Ende der Achse $f$ sitzenden, oben erwähnten, festen Rädchen ihre Drehung auf ähnliche radiale Achsen $g$ des unteren Raumes, die ebenfalls Zahnräder und an ihren Enden Scheiben mit Ziffern tragen. Diese Ziffernscheiben liegen unter einem Vorsprung des Dosenkastens und tragen die Ziffern auf ihrer Stirnfläche, die in runden Schaulöchern (bei Bl.) von oben aus sichtbar werden.

Die Verlegung des Zählwerkes in den untern Teil der Dose hat für die Zehnerübertragung Bedeutung. Beim Übergang einer Ziffernscheibe von 9 auf 0 wird nämlich, wenn soeben die Achse $f_{i}$ mit ihrem Zahnrad $z_{i}$ die verschiedenen Zähne der Stufenscheibe passiert hat, ein hinter diesen folgender Schalthebel durch eine Vorrichtung an der Achse $g$ der Ziffernscheibe von unten aus in die Höhe gedrückt, so daß für das folgende Zahnrad $z_{i+1}$ außer den festen Zalinstufen nun noch dieser Schalthebel als Zahn wirkt. 
Dann folgt auf der Stufenscheibe ein Anschlag, durch den der Schalthebel wieder niedergedrüekt und gleichzeitig der frühere Zustand im Zählwerk hergestellt wird.

Zwischen den Achsen $g$ der Ziffernscheiben liegen andere Achsen $h$, welche die Umdrehungen der Kurbel (Multiplikator bzw. Quotient) zäblen und ebenfalls Ziffernscheiben tragen, deren Schaulöcher zwischen den andern Schaulöchern (die das Produkt oder den Dividenden zeigen) liegen. Durch einen auf dem Vorsprung der Dose aufliegenden, verschiebbaren Ring ist immer die eine Art Ziffern verdeckt, die auch noch durch rerschiedene Farbe von der andern Art unterschieden ist.

Die Subtraktion (Division) wird bei Kurbeldrehung in demselben Sinne ausgeführt, nachdem ein Stellknopf auf dem Deckel von Addition auf Subtraktion verschoben ist.

18. Sellings Maschine. Auf einem ganz andern Prinzip wie die vorher beschriebenen beruht die Multiplikationsmaschine von E. Selling in Wiirzburg (1887), das hier nur in seinen Hauptzügen erläutert werden möge. $\left.{ }^{1}\right)$ Hat man eine Nürnberger Schere ${ }^{2}$ ) und hält man den Anfangspunkt 0 fest, verschiebt den Punkt 1 um eine Strecke $u$, so verschiebt sich der Punkt 2 um $2 u$, allgemein $n$ um $n w$, da bei der Streckung die Diagonale jedes Parallelogramms sich um die gleiche Länge vergröBert. Selling hat 10 Kreuzungspunkte, er benutzt aus praktischen Gründen als festen Punkt einen mitt. leren. Da dies unwe-

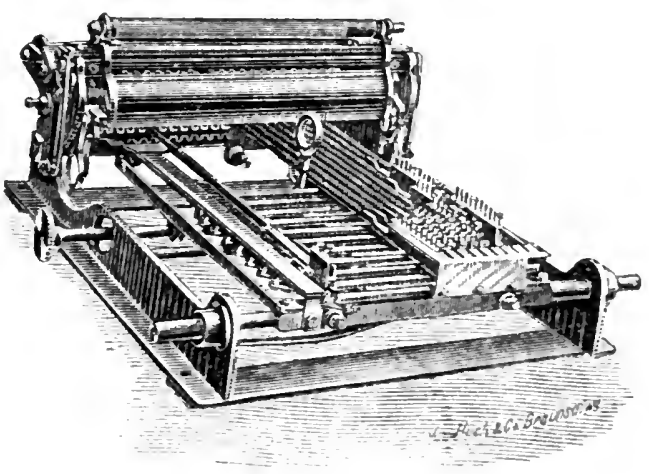

Fig. 17 .

1) E. Selling, Eine neue Rechenmaschine, Berlin 1887. Hartmann, Zeitschr. f Instr. $1857,403$.

2) Sie gleicht einer Kette ron Parallelogrammen, deren in der Mittelrichtnng liegende Ecken von den Kreuzungspunkten der Arme der einzelnen Scheren gebildet werden. Die Arme der auf einander folgenden Scheren hängen in Gelenken zusammen, welche die anderen Eckpunkte der Parallelogramme bilden. 
sentlich ist, denken wir uns wie vorher Punkt 0 fest und Punkt 1 durch einen Handgriff verschoben und die Verschiebung $w$ an einer Skala gemessen. Mit der Schere wird eine in ihrer Längsrichtung liegende Zahnstange in Verbindung gesetzt, die, wenn der Punkt $n$ mit ihr verbunden wird, die Verschiebung $n w$ erfährt. Diese Zahnstange greift in ein senkrecht stehendes Zahnrad ein und dreht es um die entsprechende Zahl von Zähnen weiter. Um eine größere Anzahl von Zahnstangen gleichzeitig in Bewegung zu setzen, sind 2 ganz gleiche Nürnberger Scheren vorgesehen, deren gleichnamige Kreuzungspunkte durch Querschienen verbunden sind, so daB sich also die $n^{\text {te }}$ Schiene um $n w$ verschiebt. Verbindet man die Zahnstangen in der gewünschten Weise mit den Schienen, so kann man jeder eine bestimmte Bewegung und jedem zugehörigen Zahnrad eine entsprechende Drehung erteilen. Die Verbindung der einzelnen Zahnstangen mit den Schienen kann man sich durch Stifte bewerkstelligt denken, die man mit der Hand durch beide hindurchsteckt, indem sie in passenden Abständen durchlocht sind. Bei der Maschine wird diese Verbindung vermittelst einer Klaviatur hergestellt, so daB man auf die betreffenden Tasten drückt, um die einzelnen Zahnstangen mit der gewünschten Schiene zu verbinden. Die Klaviatur bewirkt also die Einstellung des Multiplikandus, während jede Ziffer des Multiplikators durch Bewegung der Schere hervorgebracht wird.

Eine andere abweichende Einrichtung besteht bei der Zehnerübertragung ${ }^{1}$ ), die im Gegensatz zu andern Maschinen immer mit dem gleichen, geringen Kraftaufwande vor sich geht. Sie findet kontinuierlich statt, ähnlich wie bei der İ̉bertragung der Bewegung des Sekundenrades einer Uhr auf das Minuten- und Stundenrad. Bei den Ziffernrädern stehen daher die Zahlen nicht in gerader Richtung, sondern erscheinen etwas verschoben, wenn das vorhergehende Rad einen Bruchteil einer ganzen Umdrehung zurückgelegt hat, ähnlich wie der Minutenzeiger zwischen 2 Strichen steht, wenn der Sekundenzeiger der Uhr nicht auf 0 zeigt. Die Übertragung erfolgt dadurch, daß die Wellen der Zahnräder selbst gezahnt sind und ein anderes kleineres Rad in Drehung versetzen, welches von innen in ein anderes neben dem ersten liegendes größeres Rad eingreift, das mit dem folgenden Zahnrad verbunden ist.

1) Hier ist nur die ursprüngliche Konstruktion berücksichtigt, bei der neueren geschieht die Kehnerübertragung durch ein System von Storchschnäbeln bzw. Planetenrädern. 
Durch geeignete Wahl der Radien dieser Räder läßt es sich erreichen, daB jedes folgende um 1 weiter rückt, wenn das vorhergehende um 10 sich gedreht hat.

Bei der Multiplikation mit mehrstelligen Multiplikatoren muB, ähnlich wie sonst, eine Verschiebung des Scherenwerks gegen das Räderwerk um je eine Stelle vorgenommen werden.

Selling hat auch eine automatische Aufzeichuung des Ergebnisses eingeführt, indem die Ziffern der Zifferräder erhaben sind und auf dem Papier einer Walze abgedruckt werden; unter dem auf die Walze gespannten Papier liegt das Farbe gebende Papier, so daß die Ziffern auf der Rückseite des weißen Papiers in richtiger Lage erscheinen.

19. Multiplikationsmaschine Millionär. Aus der Beschreibung geht hervor, daB die Sellingsche Maschine sich ron den vor-

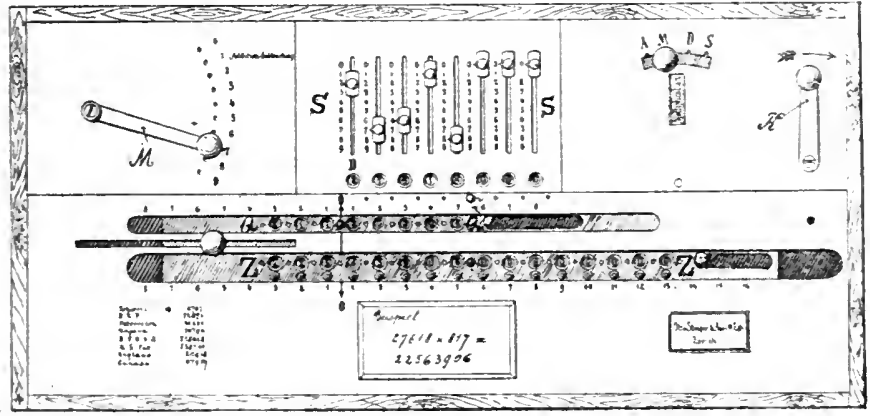

Fig. 18.

hergehenden dadurch unterscheidet, daB sie Multiplikationen ausführt. Die erste eigentliche Yultiplikationsmaschine stammt, wie bereits erwähnt, von Bollée (1888), aber in den Handel gekommen ist wohl von Multiplikationsmaschinen nur die von Otto Steiger erfundene, die von $\mathrm{Hans} \mathrm{Egli}$ in Zürich konstruiert und Millionär benannt ist.

Auf der Deckplatte erblickt man zwei Teile, die wir als vorderen und hinteren unterscheiden wollen. Der letztere (der obere in der Figur 18) zerfällt wieder in drei Abteilungen, deren mittelste die uns bereits bekannten (hier 8 ) Einstellschlitze enthält, vor denen in Schaulöchern sich die mit den Knöpfen eingestellten Zahlen durch Stahlbänderiibertragung noch einmal zeigen. In der Abteilung rechts davon befindet sich die Umstellungseinrichtung, bei der ein Knopf 
auf $A$ (Addition), $M$ (Multiplikation), $D$ ) Division) oder $S$ (Subtraktion) geschoben wird und gleichzeitig das betreffende Wort sichtbar wird. In derselben Abteilung noch weiter rechts sehen wir die Kurbel, die immer nur in der Richtung des Uhrzeigers gedreht wird und vor jeder andern Operation in ihre Anfangsstellung einschnappen muB. Der Multiplikationshebel $M$ in der linken hinteren Abteilung wird bei Addition und Subtraktion auf 1 gestellt, für Multiplikation und Division auf die betreffende Zahl des Multiplikators und Divisors. Der vordere Teil der Deckplatte zeigt zwei Reihen von Schaulöchern, in der hinteren, der Kontrollreihe $Q Q$ erscheint der Multiplikator (Quotient) in 8 Schaulöchern, während in den 16 Schaulöchern der vorderen, der Resultatreihe $Z Z$, das Produkt (Dividend) sich zeigt. Für jede dieser Reihen ist eiue Auslöschvorrichtung vorhanden, durch deren seitliche Bewegung sämtliche Ziffernscheiben auf Null gestellt werden. Dieser vordere Teil kann durch Druck auf einen Knopf, die Wagenschiebevorrichtung, von Hand hin- und hergeschoben werden, um die Zahlen der Kontroll-und Resultatenreihe in einer der 8 möglichen Stellungen zu erhalten. Bei der Multiplikation (und Division) schiebt der Wagen sich nach jeder Kurbeldrehung selbsttätig um eine Stelle nach links weiter.

Man unterscheidet drei Hauptmechanismen bei dieser Maschine: den Multiplikationsmechanismus, den Übertragungsmechanismus und das Registrierwerk.

Das Verständnis des Multiplikationsmechanismus wird durch das Rechenverfahren mit den Neperschen Rechenstäben ${ }^{1}$ )

1) Die Bacilli Neperiani oder Virgulae numeratrices (von J. Blater wird eine "Erleichterungstafel" in

\begin{tabular}{|c|c|c|c|c|c|c|}
\hline Jndex & \begin{tabular}{l|l|}
0 & 4 \\
\end{tabular} & 3 & 4 & 2 & 9 & 4 \\
\hline 2 & 00 & & 8 & $0>$ & & $0^{8}$ \\
\hline 3 & 0 & & & 0 & & $1^{2}$ \\
\hline 4 & $2 0 \longdiv { 1 6 }$ & 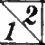 & & $0^{8}$ & $3^{6}$ & 16 \\
\hline 5 & $0 \sqrt{2}$ & 15 & 29 & 10 & $4^{5}$ & 20 \\
\hline 6 & $\log _{2} 4$ & 8 & 24 & 12 & 54 & $2^{4}$ \\
\hline 7 & 0028 & $?$ & 28 & 1 & $6^{3}$ & 28 \\
\hline 8 & $0 \sqrt{3}$ & 24 & $3^{2}$ & 16 & $7^{2}$ & $33^{2}$ \\
\hline 9 & 0 & 8 & 8 & 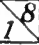 & 1 & 36 \\
\hline
\end{tabular}
den Handel gebracht, auch andere ältere und neuere Formen sind im Gebrauch) sind vierkantige Stäbchen, auf denen jede der 4 Seitenflächen das einfache Einmaleins für die jedesmal am Kopfe stehende Zahl enthält. Durch Zusammenstellung verschiedener Stäbchen findet man das 2 bis 9 fache beliebiger mehrstelliger Zahlen, wenn man die durch Diagonallinien getrenntes, schräg untereinander stehenden Zahlen addiert. Wir berechnen $\mathrm{z}$. B. die Vielfachen von $M=0,434294$ (Modulus) mit"Beschränkung auf 6 Dezimalstellen. Wir suchen die Stäbchen bzw. diejenigen ihrer Seitenflächen aus, welche die Ưber- 
erleichtert. Stellt man z. B. an den 8 Schlitzen $S S$ die Zahlen 00434294 ein, die gleichzeitig in den Schaulöchern erscheinen, so stellt man, um das 7 fache der Zahl zu erhalten, den Knopf auf $M$ und den Hebel $\boldsymbol{M}$ auf $\boldsymbol{\tau}$ ein. Nach dem ersten Viertel einer Umdrehung der Kurbel $K$ erscheinen dann in den Schaulöchern der Reihe $Z Z$ die Ziffern 00222162 , die während des weiteren Verlaufs derselben Kurbeldrehung infolge der selbsttätigen Wagenverschiebung um eine Stelle nach links rücken: 02221620. Dann wird die Zahlenreihe 00818438 zunächst ohne Zehnerübertragung hinzuaddiert, so daB 02039058 erscheint, erst beim letzten Viertel der Drehung tritt die Zehnerübertragung (ron den durch den Druck hervorgehobenen Zahlen nach links wirkend) in Erscheinung, so daB 03040058 entsteht. In der Kontrollreihe $Q Q$ zeigt sich der Multiplikator: 00000007.

Wir sehen uns jetzt die inneren Einrichtungen an, um diese Vorgänge zu begreifen. Unter jedem der 8 Schlitze $S$ befindet sich parallel mit ihm je eine vierkantige Achse, auf der ein Zabnrad $z$ hin- und hergleitet, der Stellung des Knopfes an der Einstellung entsprechend. Am vorderen, dem Beschauer zugewandten Ende befinden sich an den Achsen festsitzende, in einer Ebene liegende Räder, welche die Drehung der Achsen auf die Zählscheiben des vorderen, beweglichen Teils der Maschine (des Registrierwerks), ähnlich wie bei der Thomasschen Maschine, übertragen. Die verschiebbaren Räder $z$ greifen ron oben in 9 unter ihnen quer zu den Schlitzen liegende Zahnstangen $Z$ ein, ron denen $Z_{1}$ unter den Ziffern 1 sämtlicher Schlitze, allgemein $Z_{i}$ unter $i=1,2 \ldots 9$ liegt. Nur unter den Nullen liegt keine Zahnstange. Diese untereinander gleichen Zahnstangen $Z$ sind in ihrer Längsrichtung verschiebbar.

Die Bewegung der Zahnstangen $Z$ geschieht nun durch den sogenannten Einmaleinskörper, der links hinten unter dem Multiplikationshebel $M$ liegt. Wir denken uns inn dureh horizontale Schuitte in 9 Zungenplatten zerlegt, von denen die erste zu unterst, die neunte zu oberst liegt. Auf der.linken Seite liegen die Endflächen aller Zungenplatten in einer senkrechten Ebene, wäl-

schriften $0,4,3,4,2,9$ und 4 tragen, legen noch den Indexstab mit den Zahlen 2 bis 9 davor und lesen das siebenfache ron II ab: 
rend sich die einzelnen Zungen nach rechts in verschiedener Länge in der Richtung auf die Zahnstangen hin erstrecken.

Jede Zungenplatte stellt das Einmaleins der Zahl dar, mit der wir sie numeriert haben. Die Längen der horizontal nebeneinander liegenden, im Querschnitt gleichen Zungen sind von der gemeinsamen Grundfläche aller Platten aus gerechnet, der Reihe nach, von hinten nach vorn geordnet:

bei der 1. Platte: 0,$1 ; 0,2 ; 0,3 ; 0,4 ; 0,5 ; 0,6 ; 0,7 ; 0,8 ; 0,9$.

$" \quad$ " 2. $\quad 0,2 ; 0,4 ; 0,6 ; 0,8 ; 1,0 ; 1,2 ; 1,4 ; 1,6 ; 1,8$.

$" \quad$ "3. " 0,$3 ; 0,6 ; 0,9 ; 1,2 ; 1,5 ; 1,8 ; 2.1 ; 2,4 ; 2,7$.

$" \quad$ " 4. " 0,$4 ; 0,8 ; 1,2 ; 1,6 ; 2,0 ; 2,4 ; 2,8 ; 3,2 ; 3,6$ usf.

Die Zeichen haben wir nur dazwischen gesetzt, um die Anordnung besser zu veranschaulichen. Wir sehen, daB in jeder Platte auf eine Zehnerzunge eine Einerzunge folgt. In Wirklichkeit existieren nicht verschiedene Platten, sondern der ganze Körper ist aus einem Stück gearbeitet.

Dieser Einmaleinskörper läßt sich nun in drei zueinander senkrechten Richtungen verschieben, die wir als räumliche Koordinatenrichtungen unterscheiden können. Der Hebel $M$ besorgt durch ein Hebelwerk, das mit Kegelrädern und Zahnstangen arbeitet, die Bewegung auf und abwärts (Richtung der $z$-Achse). Wird $M$ auf 0 gestellt, so nimmt der Körper die tiefste ihm mögliche Stellung ein, er liegt dann ganz unterhalb der Ebene, in der sich die Zahnstangen befinden. Stellt man aber z. B. $M$ anf 4 , so liegt die vierte Zungenplatte den schmalen Enden der Zahnstangen gegenüber. Die erste Vierteldrehung der Kurbel $K$ bewirkt nun eine Bewegung des Multiplikationskörpers von links nach rechts auf die Zahnstangen hin (Richtung der $x$-Achse). Die Lage des Einmaleinskörpers ist hierbei so, daß die Zehnerzungen an die Zahnstangen stoßen, die Einerzungen aber in die Zwischenräume zwischen den Zahnstangen zeigen. Ist z. B. wieder 00434294 an den Schlitzen $S$ eingestellt, und soll diese Zahl mit 4 ( $M$ auf 4 ) multipliziert werden, so stehen die 9 Zehnerzungen der vierten Platte mit den Längen $0,0,1,1,2, \cdot 2,2,3,3$ von hinten nach vorn geordnet den Zahnstangen 1 bis 9 gegenüber. Wir erhalten folgende Übersicht (von oben gesehen in der $x-y$-Ebene) ${ }^{1}$ ):

1) Unter den Einstellknöpfen 0, 0 liegt keine Zahnstange; über den Zahnstangen $1,5,6,7,8$ befinden sich im Beispiel keine Einstellknöpfe, in diese Zahnstangen greifen daher auch keine Zahnräder ein, die sich ja unter den Knöpfen befinden. 


\begin{tabular}{|c|c|c|}
\hline Zungenlängen & Zahnstangen & Einstellungen $(S)$ \\
\hline der Platte 4. & - & $\begin{array}{llll}0 & 0 & \ldots\end{array}$ \\
\hline 0 & 1 & ........ \\
\hline 0 & 2 & .2. \\
\hline 1 & 3 & $.3 .$. \\
\hline 1 & 4 &. .4 .4 .44 \\
\hline 2 & 5 & . . . . . . \\
\hline 2 & 6 & ....... \\
\hline 2 & 7 & ........ \\
\hline 3 & 8 & ...... \\
\hline 3 & 9 & . . \\
\hline & & $\begin{array}{llllllll}0 & 0 & 4 & 3 & 4 & 2 & 9 & 4 \\
0 & 0 & 1 & 1 & 1 & 0 & 3 & 1 .\end{array}$ \\
\hline
\end{tabular}

Die Zungen und die Zahnstangen sind ron links nach rechts gerichtet $(x$-Achse). Durch die Rechtsbewegung des Einmaleinskörpers wird, wie man sieht, keine Verschiebung der Zahnstange 2 verursacht, da die gegenüberliegende Zungenlänge 0 ist, dagegen werden die Zahnstangen 3 und 4 je um eine Einheit, die Zahnstange 9 um 3 Einheiten nach rechts verschoben. Gleichzeitig werden die darüber gestellten Zahnräder um dieselbe Anzahl von Zähnen gedreht, welche die Reibe $Z Z$, deren Scheiben vorher auf 0 standen, anzeigt.

Die Kurbel $K$ setzt ein Kegelrad in Bewegung, in das ein anderes mit halb so grobem Radius eingreift, so daB dieses bei einmaliger Kurbeldrehung zwei Umdrehungen vollführt. Dieses zweite Rad setzt aber mittels einer mit ihm durch einen Kurbelzapfen verbundenen Pleuelstange und eines Schlittenwerkes den Einmaleinskörper in Bewegung und schiebt ihu daher bei einer einzigen Kurbeldrehung zweimal hin und her (in der Richtung der $x$-Achse) um eine durch den Durchmesser des rom Kurbelzapfen beschriebenen Kreises bestimmte Länge. Bevor nun die zweite Bewegung des Einmaleinskörpers gegen die Zahnstangen beginnt, findet durch einen ebenfalls durch die Kurbel $K$ betätigten Mechanismus eine Bewegung des Körpers in der dritten Koordinatenrichtung ( $y$-Richtung), vom Beschauer gesehen, nach hinten statt, die so bemessen ist, daB jetzt die Einerzungen den Zahnstangen gegenübertreten, während die Zehnerzungen in die Zwischenräume zwischen den Zahnstangen gerichtet sind, also unwirksam werden. ${ }^{1}$ ) Ferner ist vorher auch eine Verschiebung des Wagens um eine Stelle nach links ebenfalls durch die Kurbeldrehung erfolgt. In unserm

1) In der folgenden Übersicht sind die längen der Zehnerzungen in Klammern nochmals beigefügt, obgleich sie nicht in Betracht kommen. 
Beispiel zeigt sich also unmittelbar vor der zweiten Rechtsbewegung des Einmaleinskörpers folgendes Bild:

\begin{tabular}{|c|c|c|c|c|c|c|c|}
\hline Zungenlängen & Zahnstangen & Ein & ast & ellu & ang & & $(S S)$ \\
\hline $\begin{array}{c}\text { der Platte } 4 . \\
(0)\end{array}$ & fehlt & 0 & 0 & & . & . & . \\
\hline $\begin{array}{c}4 \\
\cdot \quad(0)\end{array}$ & 1 & . & . & - & . & - & $\cdot \cdot$ \\
\hline $\begin{array}{c}8 \\
(1)\end{array}$ & 2 & . & . & • & . & 2 & $\cdot \cdot$ \\
\hline $\begin{array}{l}2 \\
(1)\end{array}$ & 3 & . & . & . 3 & . & . & $\cdot \cdot$ \\
\hline $\begin{array}{c}6 \\
(2)\end{array}$ & 4 & . & . & 4 . & 4 & . & . 4 \\
\hline $\begin{array}{c}0 \\
(2)\end{array}$ & 5 & . & · & · & $\cdot$ & • & $\cdot \cdot$ \\
\hline $\begin{array}{c}4 \\
(2)\end{array}$ & 6 & . & . & . . & . & . & $\cdot$ \\
\hline $\begin{array}{c}8 \\
(3)\end{array}$ & 7 & . & . & • & . & • & $\cdot \cdot$ \\
\hline (3) & 8 & . & . & . & . & · & $\cdot \cdot$ \\
\hline 6 & 9 & 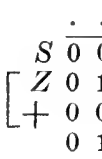 & $\begin{array}{l}. \\
0 \\
1 \\
0 \\
1\end{array}$ & $\begin{array}{l}. \\
43 \\
1 \\
2 \\
3\end{array}$ & $\begin{array}{l}. \\
4 \\
0 \\
6 \\
6\end{array}$ & $\begin{array}{l}. \\
2 \\
3 \\
8 \\
1\end{array}$ & $\begin{array}{ll}9 & . \\
9 & 4 \\
0^{1} & . \\
6 & 6 \\
7 & 6 .\end{array}$ \\
\hline
\end{tabular}

Die Vorwärtsbewegung der Zungen und die dadurch bewirkte Verschiebung der Zahnstangen und Drehung der Achsen der eingreifenden Zahnräder verändert die Ziffern von $Z$ um die mit + hinzugefügte Zahl, so daB die Endzahl herauskommt. Beim letzten Viertel der Kurbeldrehung tritt die Zehnerübertragung ein, so daB 01737176 erhalten wird.

Hiermit haben wir die wesentlichen Bestandteile dieser zurzeit wohl vollkommensten Multiplikationsmaschine hervorgehoben, die andrerseits durch ibre Größe etwas unhandlicher ist.

20. Differenzenmaschinen. Zur Berechnung von Tafelwerken ist die unbedingte Sicherheit einer Rechenmaschine von solcher Bedeutung, daß sich trotz der großen Kosten die Konstruktion einer besonders hierfür geeigneten Maschine lohnt. Dieses Problem hat insbesondere $\mathrm{Ch} . \mathrm{Babbage}^{2}$ ) um 1812 in Angriff genommen,

1) Gegen oben um eine Stelle verschoben.

2) Das Buch von Charles Babbage, Passages from the life of a Philosopher, London 1864 ist z. T. sehr amüsant geschrieben. Der 
doch ist sein Werk nur teilweise zur Vollendung gekommen. Dagegen sind die von G. und E. Schütz und von M. Wiberg ${ }^{1}$ ) ersonnenen Maschinen zur nutzbringenden Verwendung gekommen. In neuester Zeit ist eine Differenzenmaschine von Ingenieur $\mathrm{Ha}$ mann in Berlin-Friedenau nach Angaben von J. Peters für die Herstellung einer logarithmisch-trigonometrischen Tafel mit 8 Dezimalstellen (herausgegeben von Bauschinger und Peters, Leipzig 1910) konstruiert worden, welche die Resultate gleichzeitig druckt (Fig. 19 a). Sie besteht aus zwei gleichartigen, voneinander unabhängigen Rechenmaschinen I und II zu je 16 Stellen nebst dem zugehörigen Druckwerk III. Sie ist auf die Rechnung mit zweiten Differenzen zugeschnitten, die bei genügend engem Intervalle der zu berechnenden Funktionen für längere Reihen konstant bleiben.

Nimmt man an, daB in einem bestimmten Zeitpunkte das Zählwerk der Maschine II den Funktionswert, das Zählwerk der Maschine I die erste Differenz anzeigt und da $B$ letztere gleichzeitig in Schaltwerk II enthalten ist, so wird die zweite Differenz im Schaltwerk I eingestellt, durch Drehung einer Kurbel $a$ zur ersten Differenz. in Zählwerk I und Schaltwerk II zugefügt, und diese durch eine zweite Kurbel $b$ dem Funktionswert (im Zählwerk II) zugelegt, durch Kurbeln wird das Druckwerk betätigt.

Die umstehende Figur $19 \mathrm{~b}$ gibt ein Bild des Vorganges, wie er an jeder der 16 Stellen stattfindet. An dem Knopf, der die Achse 1 dreht, wird die zweite Differenz auf der durch ein Schauloch ablesbaren Scheibe 5 eingestellt. Durch die Drehung der Kurbel $a$ wird erstlich die Doppelzahnstange 23 in ihrer Längsrichtung verschoben und dadurch das auf der festen Achse 9 drehbar gelagerte Zahnrad 10 herumgedreht, bis durch Übertragung seiner Umdrehung auf das Zahnrad 4, mit dem sich die Achse 1 dreht, im Schauloch der Scheibe 5 eine Null erscheint. Durch Fehlen eines Zahnes bei 4 läBt sich diese Begrenzung der Bewegung erreichen, indem dann 10 nicht mehr eingreift. Dieselbe Kurbelbewegung (a) hat die Achse 24 um den Zapfen 25 gedreht und Rad 7 so weit nach rechts verschoben, daß vermöge einer Flansche die Achse 3 an den Drehungen von Achse 1 teilnimmt. Es möge hier gleich bemerkt werden, daB mit dieser Achse die Ziffern-

Bericht über die Differenzenmaschine sowie über die spätere analytische Maschine zieht sich wie ein roter Faden durch das Werk.

1) M. Wiberg, Logarithm-Tabeller, Stockholm 1875 siud mit der Maschine gerechnet. 


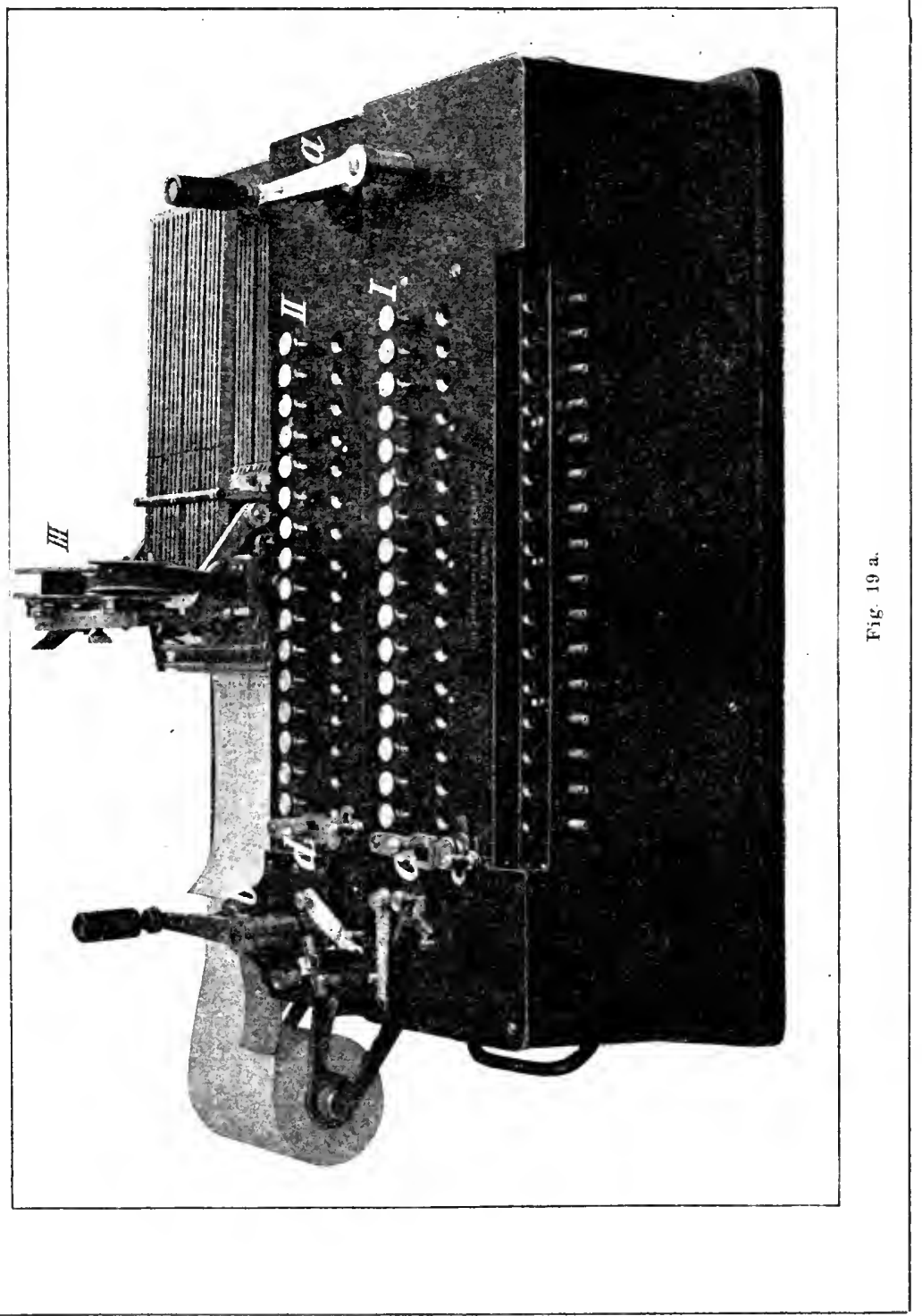




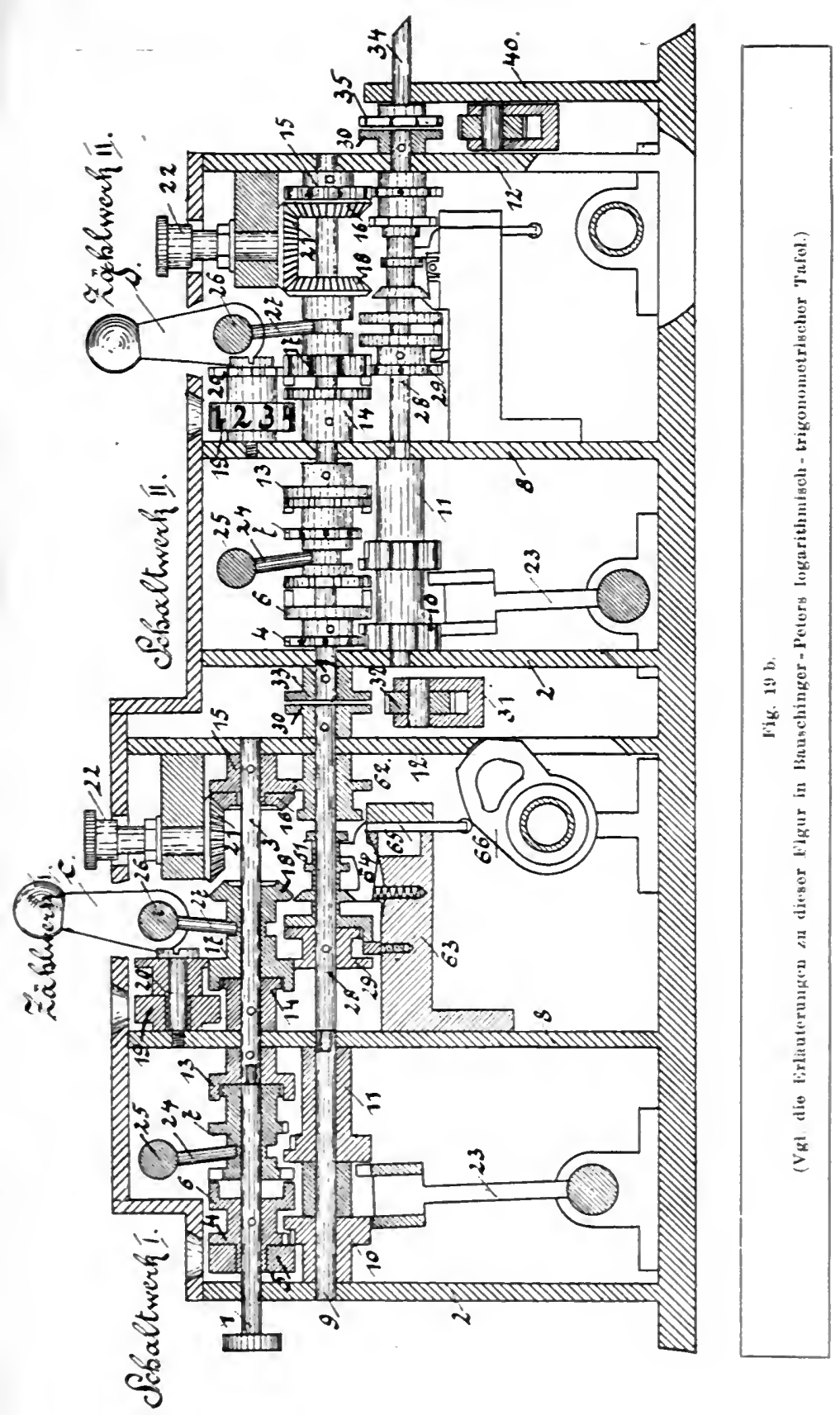


III. MeBrolle.

scheibe 19 des Zählwerkes I in Verbindung steht. Nach der Hälfte der Drehung der Kurbel a kippt die Zahnstange 23, die sich um eine Achse dreht, von Rad 10 hinweg und nach Rad 11 hinüber, durch welches Rad 7 auf Achse 1 um ebensoviel Zähne vorwärts gedreht wird, als es vorher zurückbewegt wurde. Da aber inzwischen Achse 24 Rad 7 mit Rad 4 verkoppelt und von der Verbindung nach rechts gelöst liat, so wird Rolle 5 wieder auf die frühere Einstellung gebracht. Auf Rolle 5 ist also der frühere Wert eingestellt, während im Zählwerk I, das ja nur an der ersten halben Bewegung teilgenommen hatte, der Wert der zweiten Differenz hinzugefügt ist. Nun hat aber die Achse 3 ihre Bewegung gleichzeitig auch auf Achse 28 übertragen, die mit der Achse 1 der Maschine II gekoppelt ist. Diese Achse trägt also keinen Einstellknopf, sondern wird von Achse 28 der Maschine I um ebensoviel gedreht, als die Änderung im Zählwerk I beträgt. Sonst ist die Einrichtung von II entsprechend, nur kommt die hier nicht ohne besondere Figuren erklärbare Druckvorrichtung hinzu. Die Hebel $s$ und $d$ dienen zur Umschaltung vou Addition auf Subtraktion.

21. Anforderungen an eine Rechenmaschine. Zum Schlusse stellen wir als Anforderungen an eine moderne Rechenmaschine die folgeuden auf:

Durchgehende Zehnerübertragung sowohl im Zählwerk (bzw. Warnungsglocke) als auch im Quotienten.

Automatischer Stellentransport.

Auslöschvorichtung für die Einstell-und die Resultatenreihen.

Ein Druckwerk, welches wenigstens das Resultat druckt, wenn möglich auch die Aufgabe (aber nicht vom Einstellwerk aus).

\section{Abschnitt.}

\section{Die Meßrolle.}

22. Einrichtung und theoretische Grundlagen. Eine Rolle, d. h. eine kreisrunde Scheibe, die auf einer ebenen Fläche, sich um ihre Achse drehend, fortbewegt wird, kommt bei einer großen Anzahl von Instrumenten zur Verwendung. Ist ihr Rand scharf, so hat sie die Eigenschaft, eine bestimmte Richtung inne zu halten, aus der sie nur durch Drehung um ihren Auflagepunkt, den Berülırungspunkt mit der Zeichenebene, in eiue andere Ricl- 
tung übergeführt werden kann. Rollen von dieser Beschaffenheit oder auch an ihrer Stelle Schneiden von etwas gekrümmter Form werden bei Planimetern und Integraphen in Anwendung gebracht.

Eine noch häufigere Benutzung erfahren Rollen mit einem flachen oder auch etwas abgerundeten Rande als MeBrollen. Sie sind dann entweder mit einer Teilung an ihrem Rande versehen, die mit Hilfe eines an der Drehung nicht teilnehmenden Index abgelesen wird, oder ihre Achse steht mit einem Zählwerk in Verbindung, welches die vollen Umdrehungen der Rolle und deren Bruchteile angibt. Die Rollenumdrehungen, die man auf diese Weise abliest, messen bei bekanntem Halbmesser der Rolle den von ihrem Mittelpunkt zurückgelegten Weg. Bezeichnen wir den Halbmesser der Rolle mit $\varrho$ und nehmen wir an, das sie sich beim Durchlaufen einer geradlinigen Strecke $s$ um den Winkel $\omega$ dreht, so beschreibt ein Punkt ihres Umfanges den Bogen $\rho \omega=\sigma$, welcher der durchlaufenen Strecke $(\sigma=s)$ gleich ist. Für unendlich kleine Wegstrecken $d s$ gilt die Gleichung $o \cdot d \omega=d \sigma=d s$, aus der für ein Kurvenstück ron der Länge $S$ zwischen zwei Punkten $A$ und $B: \varrho \int_{\omega_{1}}^{\sigma_{2}} d \omega=\rho\left(\omega_{2}-\omega_{1}\right)=\sigma_{2}-\sigma_{1}=S$ folgt. Wir nennen $\sigma$ bzw. $d \sigma$ auch die Abwicklung der Rolle.

Eine Voraussetzung für die Zuverlässigkeit der Messung ist die, daB die Rolle, die mit einem sanften Druck gegen die Unterlage gedrückt wird, sich in jedem Augenblick wirklicb dreht und nicht gleitet. Ein Gleiten der Rolle, bei dem sie sich nicht dreht, sondern stets in demselben Punkte die Unterlage berührt, muB dagegen stattfinden, wenn eine Verschiebung der Rolle in der Richtung ihrer Achse vorkommt. Wenn die Richtung der Rollenbewegung oder was dasselbe ist, die Ebene der Rolle nicht mit der Richtung des Weges (bei Kurren mit der Tangente) zusammenfallt, sondern beide Richtungen einen Winkel $\alpha$ mit einander bilden, so kann man sich die Rollenbewegung in zwei Bewegungen zerlegt denken, von denen die eine senkrecht zur Rollenachse, die andere in der Richtung der Rollenachse sich vollzieht. Die Abwicklung ist dann nicht dem zurückgelegten Wege gleich, sondern gibt nur die Komponente, die senkrecht zur Rollenachse liegt, daher ist $d \sigma=d s \cdot \cos \alpha$.

Wir schaiten noch ein, $d a B$ es nicht notwendig ist, daB die Rolle senkrecht zur Unterlage steht, sondern daB ihre Abricklung 
dieselbe bleibt, wenn ihre Ebene gegen die Unterlage geneigt ist, so lange nur eine ungehinderte Drehung stattfindet und keine Reibung sie verhindert.

23. Verschiedene Mechanismen und Ersatz der Rolle durch Zylinder usw. Da es ferner nur auf die relative Bewegung ankommt, so kann die Rolle auch fest stehen und die Unterlageebene unter ihr verschoben werden. Ein derartiger Fall kommt vor, wenn es sich darum handelt, eine geradlinige Bewegung in eine kreisförmige zu verwandeln. Hierbei ruht die Rolle auf einer rotierenden Kreisscheibe auf, wobei ihre Achse meist nach dem Mittelpunkt der Scheibe gerichtet ist. Wenn die Entfernung des Auflagepunktes vom Mittelpunkt $e$ ist und die Scheibe sich-um einen kleinen Winkel $d \varphi$ dreht, so ist die Abwicklung der Rolle $d \sigma=e d \varphi$.

Sind $\varphi_{1}$ und $\varphi_{2}$ die Winkel, welche ein Scheibenhalbmesser $\varrho$ vor und nach der Drehung mit einer beliebig gewählten Anfangsrichtung einschließt, und $\omega_{1}$ und $\omega_{2}$ die entsprechenden Ablesungen an der Rolle, so erhält man $\left(\omega_{2}-\omega_{1}\right)=\frac{e}{\rho}\left(\varphi_{2}-\varphi_{1}\right)$ oder auch $\sigma_{2}-\sigma_{1}=e\left(\varphi_{2}-\varphi_{1}\right)$, wofür wir kurz $\sigma=e \varphi$ schreiben wollen. Häufig wird $e$ veränderlich sein, indem etwa die Drehung $d \varphi=p \cdot d x$ der Änderung der $x$-Koordinate proportional ist, während $e=y$ der $y$-Koordinate gleich gemacht wird. Ist dann $y=f(x)$ gegeben, so wird $\sigma_{2}-\sigma_{1}=p \int_{x_{1}}^{x_{2}} y d x$ oder auch $\omega_{2}-\omega_{1}=\frac{p}{\varrho} \int_{x_{1}}^{x_{2}} y d x$.

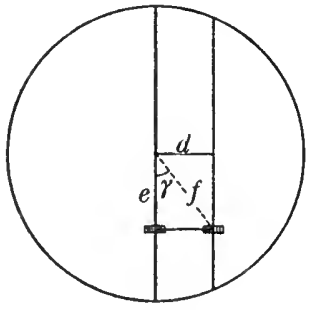

Fig. 20.

Diesen Fall werden wir z. B. beim Hansenschen Planimeter kennen lernen.

Es ist nicht nötig, daß die Achse der Rolle mit einem Durchmesser der ruhenden Scheibe zusammenfällt, és genügt, daß sie ihm parallel ist (Fig. 20). Findet nämlich eine Verschiebung längs einer Sehne statt, die den Abstand $d$ vom Mittelpunkt der Scheibe hat, ist ferner $f$ die Entfernung des Rollenauflagepunktes vom Mittelpunkt der Scheibe, $\gamma$ der Winkel, den $f$ mit dem der Rollenachse parallelen Durchmesser bildet, so ist bei einer Scheibendrehung $d \varphi$ die Abwicklung der Rolle $d \sigma=f \cdot d \varphi \cdot \cos \gamma$ oder $d \sigma=e d \varphi, d$. h. die Abwicklung ist dieselbe, als wenn die Rolle in ihrer Ebene nach dem Durchmesser 
verschoben wird, und ihre Achse nach dem Mittelpunkt der Scheibe gerichtet ist.

Diese Verbindung von Scheibe und Rolle scheint zuerst Poncelet angewendet zu haben, sie wird in der Regel als Gonellascher Integrationsmechanismus bezeichnet, da Gonella sie durch sein Planimeter allgemeiner bekannt gemacht hat.

Die Kreisscheibe kann als besonderer Fall eines Kreiskegels aufgefabt werden, wenn nämlich sein Öffnungswinkel $180^{\circ}$ beträgt. Läuft eine Rolle auf einer Kegelfläche, die sich um ihre Achse um den Win-

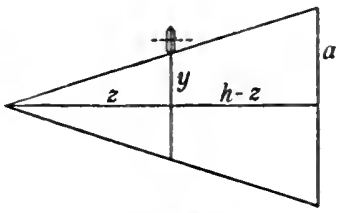

Fig. 21. kel $d \varphi$ dreht, und ist die Rollenachse der Kegelachse parallel, so möge der Berührungspunkt der Rolle sich auf dem Kreise mit dem Radius $y$ bewegen, der von der Kegelspitze den Abstand $z$ hat (Fig. 21). Dann ist die Abwicklung $d \sigma=y d \varphi$ oder wenn $a$ der Radius des Grundkreises des Kegels und $h$ seine Höhe ist, $d \sigma=\frac{a z}{h} d \varphi$. Auch hier kann $z=\varphi(x)$ und $d \varphi=p \cdot d x$ sein, so daB man entsprechend wie früher $\sigma_{2}-\sigma_{1}=\frac{a}{h} p \int_{x_{1}}^{x_{2}} z d x$ erhält.

Die Kegelfläche ist nicht die einzige gekrümmte Oberfläche, auf der man eine Rolle laufen läBt. Insbesondere kommt auch ein Mechanismus in Betracht, wo die Rolle auf einer Kugeloberfläche läuft. Wir nehmen zunächst an, daB die Rolle senkrecht auf ihr steht, d. h. daB ihre Ebene einen gröBten Kreis $B N N^{\prime}$ aus der Kugel ausschneidet. Es sei $O$ der Yittelpunkt der Kugel, $B$ der Berührungspunkt der Rolle, der Kugelradius $O B=r$, der Rollenradius $B R=\varrho$. Die Kugel drehe sich um einen Durchmesser $P P^{\prime}$ als Achse, mit dem der Radius $O B$ den Winkel $\delta$ einschließe. Dreht sich die Kugel um

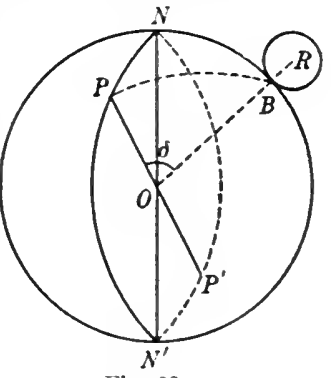

Fig. 22. ihre Achse um den Winkel $d \varphi$, so bewegt sich der Berührungspunkt $B$ auf einem kleinen Kugelkreise mit dem Radius $r \sin \delta$ und beschreibt einen Bogen $r \sin \delta d \varphi$. Die Bewegung der Rolle wird teils eine rollende, teils eine gleitende sein und nur die erstere hat eine Drehung der Rolle zur Folge. Wenn wir den Winkel $P B N^{\top}$ 
mit $\gamma$ bezeichnen, d. h. den Winkel, welchen der sphärische Radius des Berührungspunktes $B$ der Rolle mit der Ebene der Rolle oder auch mit dem größten Kreise $N B N^{\prime}$ einschließt, so beträgt die Abwicklung der Rolle $d \sigma=r \sin \delta \sin \gamma d \varphi$, da ja die Rollenebene mit der Bewegungsrichtung des Berührungspunktes (der Tangente des kleinen Kreises) den Winkel $90^{\circ}-\gamma$ einschlieBt.

Wenn die Rollenebene einen kleinen Kreis aus der Kugel ausschnitte und die Rolle infolgedessen schräg auf der Kugel stände, so wäre die Abwicklung dieselbe, nur müBte dann der Winkel $\gamma$ als der Winkel zwischen der sphärischen Tangente desjenigen kleinen Kugelkreises, den die Ebene der Rolle ausschneidet, und dem sphärischen Radius des andern kleinen Kreises, den der Berührungspunkt $B$ bei der Drehung der Kugel beschreibt, definiert werden.

Aus praktischen Gründen tritt bei vielen Instrumenten an Stelle der Rolle, die auf einer gekrümmten Fläche läuft, ein Zylinder, also gewissermaßen eine große Zahl von Rollen mit gemeinsamer Achse. An Stelle der Verschiebung einer Rolle in der Richtung ihrer Achse findet dann ein Wälzen oder Kippen des gegen die Fläche gedrückten Zylinders statt, so daß der Berührungspunkt des Zylinders sich auf ihm verschiebt und verschiedene Rollen, aus denen wir uns ihn zusammengesetzt denken, nach einander in Berührung mit der krummen Oberfläche kommen. Auch ist in einzelnen Fällen der Versuch gemacht worden, die Rolle durch eine Kugel zu ersetzen, deren Umdrehungen um eine Achse gemessen werden, und die auf einer andern Kugel rollt.

Alle diese Mechanismen mit gekrümmten Flächen bezwecken, unbeabsichtigte Bewegungen des MeBapparates zu verhindern, indem seitliche Bewegungen einer MeBrolle auf einer ebenen Fläche infolge von Unebenheiten $u$. dgl. Drehungen der Rolle nicht ausschließen, also das Ergebnis verfälschen. Bei krummen Flächen ist dagegen die Forderung, da $B$ die Berührung immer nur in einem Punkte stattfindet, leichter erfüllbar.

Insbesondere ist hierbei noch auf eine Bemerkung Rücksicht zu nehmen, die Clark Maxwell zuerst gemacht hat, daß bei einer seitlich wirkenden Kraft, die nicht genau mit der Richtung der Rollenachse zusammenfällt, die eine Komponente (senkrecht zur Rollenachse) eine Rollbewegung verursacht, wenn die Rolle in dieser Richtung rollen kann, wie dies bei ebener Unterlage der Fall ist. 


\section{Abschnitt. \\ Stetige Rechenapparate.}

Die im vorigen Abschnitte beschriebenen kinematischen Grundlagen können zur Ausführung derselben Rechenoperationen dienen, die durch Rechenmaschinen in gan\% anderer Weise zu Stande kommen.

24. Addition und Subtraktion. Hierher gehört zunächst der sog. Proportionalrechenschieber von $\mathrm{Ch}$. Hamann. Ein scharfrandiges Stahlrad $R$ wird mit seiner horizontalen Achse gabelförmig (oder in einem ringförmigen Lager wie in der Fig. 23) von einem Arm gehalten, dessen anderes Ende einen Indexstift $m$ umfaßt, der sich in einem Schlitz längs einer Skala $M I$ verstellen läBt. Das Rad trägt einen zur Richtung $M$ senkrechten, horizontal liegenden Zylinder $C$, der das Rad senkrecht über seinem Mittelpunkte berührt. Dieser $\mathrm{Zy}$ linder läBt sich in der Richtung seiner Achse verschieben und trägt einen Index $S$, dessen Stellung an einer zu $M(y$-Achse) senkrechten Skala $\boldsymbol{A}(x$-Achse) abgelesen wird. Wird $S$ auf einen bestimmten Punkt der Skala $A$ eingestellt und festgehalten, so daB die Richtung des Armes $m R$ unverändert bleibt, und der Zylinder in seiner Längsrichtung um eine bestimmte Strecke verschoben, so findet eine Drehung des Rades und gleichzeitig des auf ihm ruhenden Zylinders statt. Diese Drehung ist proportional der Einstellung $O m=y$ und wird durch eine mit der

0

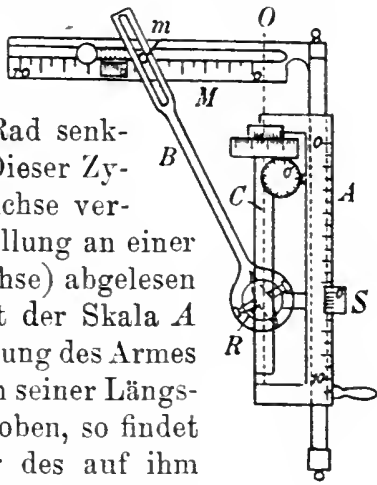

Fig. 23. Zylinderachse verbundene Zählvorrichtıng abgelesen. Bezeichnen wir nämlich den Winkel, den eine Senkrechte $R O$ auf $\boldsymbol{~} \boldsymbol{L}$, welche die konstante Lünge $l$ hat, mit der Richtung des Armes $R m$ einschlieBt, mit $\alpha$, so bewirkt eine Verschiebung $d x$ des Zylinders in der $x$-Richtung eine Drehung der Rolle um $d \omega$ und die Abwicklung der Rolle ist $\rho d \omega=d \sigma$. Durch die Rollendreliung wird eine Drehung des Zylinders $d \varphi$ um seine Achse veranlaBt, die für einen Zylinderhalbmesser $r$ eine Verschiebung $r d \varphi$ des Berïhrungspunktes auf der Zylinderfläche in der Richtung der y-Achse zur Folge hat. Der Weg des Berührungspunktes auf der Rolle ist die Resultante der beiden rechtwinkligen Komponenten $d x$ und $r d \varphi$. Es ist daher $\cos \alpha d \sigma=d x, \quad \sin \omega d \sigma=r d \varphi$ und $d \varphi=\frac{d x}{r} \cdot \operatorname{tg} \omega$. Anderer- 
$a$ mit Hilfe einer Skala einstellt, indem man $P$ auf der Achse von $A$ verschiebt, und die Rolle $A$, die ebenfalls mit einer Teilung versehen ist, von null ausgehend bis zu einer Ablesung $\omega_{1}$ dreht, so wird dadurch die Kugel und damit auch die Rolle $B$ in Drehung versetzt. Die Ablesung $\omega_{2}$ der Rolle $B$ ist dann $\omega_{2}=\frac{a}{k} \cdot \omega_{1} \cdot \frac{\varrho_{1}}{\varrho_{2}}$ wenn $\sigma_{1}=\varrho_{1} \omega_{1}, \sigma_{2}=\varrho_{2} \omega_{2}$ ist. Durch passende Wahl der Einheit $\left(\frac{\varrho_{1}}{\varrho_{2}}=k\right)$ oder der Rollendurchmesser erhält man also $\omega_{2}=a \cdot \omega_{1}$ als Produkt der eingestellten Zahlen.

Besonders vorteilhaft ist diese Vorrichtung, wenn man $a$ konstant läBt, indem man dann eine Tabelle der Produkte von $a$ mit verschiedenen Faktoren erbält.

Dreht man dagegen die Rolle $B$, so wird die Kugel ebenfalls um ibre Achse gedreht und damit auch $A$ und man erhält (wieder für $\frac{\varrho_{1}}{\varrho_{2}}=k$ ) $\omega_{1}=\frac{\omega_{2}}{a}$ als Quotienten zweier eingestellten Zahlen.

26. Quadratwurzelziehung. Wir nehmen jetzt an, daB die Achse der Rolle $A$ eine Schraube und $P$ eine Schraubenmutter sei. Durch Drehung der Rolle $A$, die durch den festen Rahmen in ihrer Stellung gehalten wird, wird die Schraube gedreht und dadurch die Schraubenmutter $P$ verschoben, die in dem beweglichen Rahmen befestigt an der Drehung nicht teilnehmen kann. Dadurch wird ferner die in dem beweglichen Rahmen sitzende Drehachse der Kugel in ihrer Lage verändert. Es seien wieder $\varrho_{1}$ und $\varrho_{2}$ die Halbmesser der Rollen $A$ und $B, d \omega_{1}$ und $d \omega_{2}$ ihre Drehungswinkel, ferner $t$ die Ganghöhe der Schraube, also auch die Änderung der Entfernung $a$, die einer Schraubenumdrehung entspricht. Daun ist $\frac{\omega_{1}}{2 \pi}={ }_{t}^{a}$, wenn $\omega_{1}=0$ für $a=0$ ist. Setzt man den Wert von $a$ in $\frac{\varrho_{2} d \omega_{2}}{\varrho_{1} d \omega_{1}}=\operatorname{tg} a=\frac{a}{k}$ ein, so folgt

$$
d \omega_{2}=\frac{\varrho_{1}}{\varrho_{2}} \frac{t}{2 \pi k} \omega_{1} d \omega_{1}
$$

und durch Integration, wenn für $\omega_{1}=0$ auch $\omega_{2}=0$ ist, $\omega_{2}=\frac{t}{4 \pi k} \frac{\varrho_{1}}{\varrho_{2}} \omega_{1}{ }^{2}$. Wählt man $V \frac{t}{4 \pi k} \frac{\varrho_{1}}{\varrho_{2}}=1$, so folgt $\omega_{1}=\sqrt{\omega_{2}}$ d. h. wenn man die Rolle $B$ um eine Zahl $\omega_{2}$ dreht, wodurch die 
Kugel und die Rolle $A$ ebenfalls in Drehung versetzt werden, so liest man an der Rolle $A$ die Quadratwurzel $V \omega_{2}$ ab.

27. Logarithmierung. Bringt man dagegen bei sonst unveränderten Verhältnissen $P$ in der Verlängerung der Drehachse der Kugel an (Fig. 25), so möge wiederum der Winkel zwischen dieser Drehachse und der Schraubenachse $A P O=\alpha$ bezeichnet werden. In diesem Falle ist $\operatorname{tg} \alpha=\frac{k}{a}$, also auch $\frac{d \sigma_{2}}{d \sigma_{1}}=\frac{\varrho_{2}}{\varrho_{1}} \frac{d \omega_{2}}{d \omega_{1}}=\frac{k}{a}$. Setzt man wieder $a=\frac{t \omega_{1}}{2 \pi}$ ein, so erhält man $d \omega_{2}=2 \pi \frac{k}{t} \frac{\varrho_{1}}{\varrho_{2}} \frac{d \omega_{1}}{\omega_{1}}$, also durch Integration

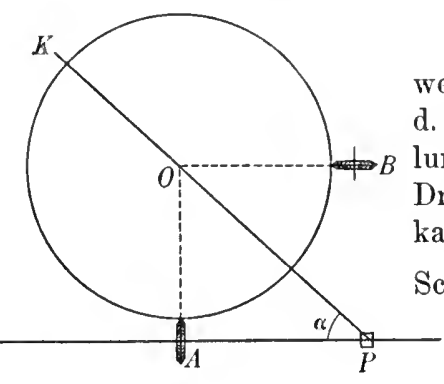

Fig. 25.

$$
\omega_{2}=2 \pi \frac{k}{t} \frac{\varrho_{1}}{\varrho_{2}} \lg \omega_{1},
$$

wenn die Integrationskonstante null ist, d. h. der Einstellung $\omega_{2}=0$ die Einstellung $\omega_{1}=1$ entspricht, was man durch Drehung der Rollen erreichen kann. Hier kann man durch passende Wahl der Schraubenganghöhe $2 \pi \frac{k}{t} \frac{\boldsymbol{\rho}_{1}}{\varrho_{2}}=$ Modulus der dekadischen Logarithmen machen. Dann wird

$$
\omega_{2}=\log \omega_{1} .
$$

Durch Drehung der Rolle $A$ um $\omega_{1}$ erhält man $\log \omega_{1}$ als Ablesung der Rolle $B$.

28. Trigonometrische und hyperbolische Funktionen. Von dem Erfinder Ch. Ha mann des unter 24. beschriebenen Proportionalrechenschiebers ist ein arithmetischer und trigonometrischer Universalrechenapparat konstruiert worden, der sich in der Konstruktion an den genannten anschlieBt. ${ }^{1}$ ) Auf einem quadratischen Metallrahmen von $17 \mathrm{~cm}$ Seitenlänge (s. Fig. 26) dreht sich ein Arm, den wir mit $A$ bezeichnen wollen um einen Punkt $D$ in der rechten oberen Rahmenecke. Wenn er der oberen Rahmenkante parallel liegt, zeigt ein an ihm befestigter Nonius auf den Nullpunkt eines bis $45^{\circ}$ reichenden Gradbogens (II). ${ }^{2}$ ) Ein zweiter Nonius wird ebenfalls

1) Zeitschrift für Instrumentenkunde 31, 101, 1911. (Beschreibung von Ingenieur K. Hoecken.)

2) Die Bezeichnung der fünf Ablesevorrichtungen ist durch die Zahlen I bis $\mathrm{V}$ von links nach rechts vorgenommen, entsprechend die der Ablesungen $a_{1}$ bis $a_{5}$. 
von ihm längs der linken Rahmenkante mitgefübrt und zeigt in der genannten Anfangsstellung auf den Nullpunkt einer geradlinigen Skala (I) an dieser Rabmenkante. An der rechten Rahmenkante befindet sich eine mit I völlig übereinstimmende Skala (V), die aber verschiebbar ist und mittels eines am Rahmen fest angebrachten Nonius abgelesen wird. Mit dieser Skala (V) ist ein um seine Achse drehbarer Zylinder (IV) so verbunden, $\mathrm{daB}$ er sich gleichzeitig mit $\mathrm{V}$ in

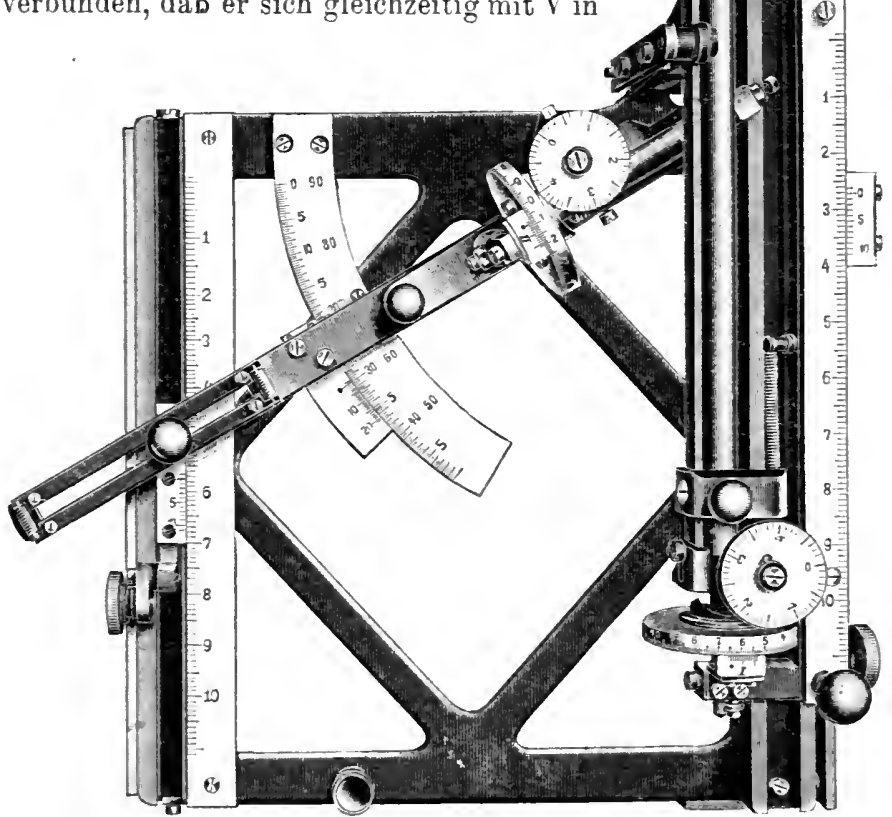

Fig. 26.

seiner Längsrichtung verschieben läBt. Nun ist auf dem Arme $A$ nahe seinem Drehpunkt $D$ ebenfalls ein Zylinder (III) gelagert, und es kann durch den Druck auf einen an der unteren Rahmenkante angebrachten Knopf bewirkt werden, daB (durch seitliche Kippung des Rahmens des Zylinders IV) die beiden Zylinder III und IV einander in einem in der Richtung des Armes $A$ gegebenen Punkte berïhren. Wird der Knopf losgelassen, so sind die Zylinder wieder außer Berïhrung. Die Drehung der Zylinder wird an zwei 
Zählwerken abgelesen, welche die ganzen Umdrehungen und ihre Bruchteile angeben.

Bezeichnet man den am Gradbogen abgelesenen Winkel mit $\alpha$, so ist der Winkel der beiden Zylinderachsen $90^{\circ}-\alpha$. Wird nun der Zylinder IV um den an der Skala V abzulesenden Betrag $a_{5}$ in der Richtung seiner Achse verschoben, so erleidet er durch die Berührung mit dem Zylinder III eine an seinem Zählwerk meßbare Drelıung $a_{4}$. Hierbei wird auch der Zylinder III eine an der Zählscheibe sichtbare Drehung $a_{3}$ erleiden. Eine Drehung des Zylinders III kann man als Resultante einer Längsverschiebung und Drehung des Zylinders IV auffassen, so daß bei konstantem Winkel $\alpha$ auch für endliche Drehungen $a_{4}=a_{3} \sin \alpha$, $a_{5}=a_{3} \cos \alpha$ ist. Wenn ferner die Rahmenseite (genauer die Entfernung des Drehpunktes $D$ von Skala I) als Einheit genommen wird, so ist die Ablesung $a_{1}$ an Skala $\mathrm{I}: a_{1}=\operatorname{tg} \alpha$.

Hieraus geht hervor, daß sich der Apparat zur Multiplikation und Division verwenden läßt, indem $a_{4}=a_{1} a_{5}, a_{5}=\frac{a_{4}}{a_{1}}$ ist, wenn man bei Herstellung der Berührung der Zylinder III und IV mit dem Druckknopf die Faktoren $a_{1}$ und $a_{5}$ auf den Skalen I und V bzw. den Dividenden am Zählwerk des Zylinders IV einstelït. Wenn man die Zählwerke, die durch eine besondere Vorrichtung auf Null gestellt werden können, eingestellt läßt, so kann man ein anderes Produkt zu einem vorhergehenden addieren usf., also was mit dem Rechenschieber nicht möglich ist, Ausdrücke von der Form $a b \pm c d \pm e f \ldots$ bilden.

Die sechs Gleichungen:

$$
\begin{array}{ll}
a_{5}=a_{3} \cos \alpha & a_{4}=a_{3} \sin \alpha \\
a_{3}=a_{4} \operatorname{cosec} \alpha & a_{3}=a_{5} \sec \alpha \\
a_{4}=a_{5} \operatorname{tg} \alpha & a_{5}=a_{4} \operatorname{ctg} \alpha
\end{array}
$$

zeigen die Möglichkeit, die Produkte einer trigonometrischen Funktion und einer Zahl zu bilden, wenn noch $\sin \left(45^{\circ} \pm \alpha\right)$ $=\cos \left(45^{0} \mp \alpha\right)$ in Betracht gezogen wird.

Wenn auberdem die Winkeldrehung des Armes $A$ durch die Längsverschiebung des Systems IV, V mittelst einer besonderen Vorrichtung hergestellt wird, so besteht für kleine Bewegungen die Differentialformel 


$$
\left.d a_{5}=\cos \alpha d a_{3} \cdot{ }^{1}\right)
$$

Da nun $d a_{5}=d_{\varkappa}$ durch passende Einrichtung der Übertragung gemacht werden kann, so folgt

$$
d a_{3}=\frac{d \alpha}{\cos \alpha} \quad \text { und } \quad a_{3}=\int \frac{d \alpha}{\cos \alpha}=\lg \operatorname{tg}\left(\frac{\pi}{4}+\frac{\alpha}{2}\right),
$$

und damit erbält man die hyperbolischen Funktionen

$$
\sin \alpha=\mathfrak{T a n g} a_{3}, \quad \sec \alpha=\operatorname{Coj} a_{3}, \quad \operatorname{tg} \alpha=\operatorname{Sin} a_{3} .
$$

Wenn man daher bei gekuppelten Zylinderwalzen die Skala V so lange verschiebt, bis die Ablesung $a_{3}$ an dem Zählwerk des Zylinders III erscheint, so hat sich der Arm $A$ von selbst auf a eingestellt. Man kann dann auf die frühere Weise das Produkt einer Zahl mit sin $\propto$ usw., also auch das Produkt einer Zahl mit Tang $a_{3}$ usf. durch den Apparat erhalten, also ebenso wie beim ungekuppelten System.

\section{Abschnitt.}

\section{Differentiatorell.}

29. Zweck. Um den Wert des Differentialquotienten $\frac{d y}{d x} \mathrm{zu}$ erhalten, also wenn $y=f(x)$ durch eine Kurve dargestellt betrachtet wird, die Richtung der Tangente, wird bei den Apparaten von Hele-Shaw das Verhältnis der Geschwindigkeiten $\frac{d y}{d t}$ und $\frac{d x}{d t}$ eingeführt und diese werden in Rotationsgeschwindigkeiten $\omega_{2}$ und $\omega_{1}$ verwandelt. In dem besonderen Falle, da $x=t$, d. h. die eine Veränderliche die Zeit und daher $\omega_{1}=\frac{d x}{d t}$ eine Konstante $(=1)$ ist, erhält man dann direkt die Geschwindigkeit $\frac{d y}{d t}=\omega_{2}$.

30. Rollen- und Scheibenmechanismus. Eine Rolle $R$ (Figur 27) möge in der Weise auf einer rotierenden Kreisscheibe $S$ angebracht sein, $\mathrm{da} B$ ihr Berührungspunkt sich längs eines Durchmessers der ruhenden Scheibe verschiebt. Diese Verschiebung findet auf einer Schraubenspindel $E$ statt, die als Achse der Rolle dient. Die Schraube werde in eine Drehgeschwindigkeit $\omega_{2}$ versetzt, die von der

1) Für das gekuppelte System ist genauer $d a_{5}=\cos (\alpha+d \alpha) d a_{3}$, wofür $(\cos \alpha-\sin \alpha d \alpha) d a_{3}$ oder mit Vernachlässigung des Gliedes zweiter Ordnung $\cos \alpha d a_{3}$ geschrieben werden kann. 
einen Veränderlichen $y$ abhängt, während die Winkelgeschwindigkeit $\omega_{1}$ der Scheibe von der anderen Veränderlichen $x$ abhängen soll. Da nun die Rolle, die wie eine Schraubenmutter auf der Spindel aufsitzt, durch die rotierende Scheibe die Geschwindigkeit $e \omega_{1}$ empfängt, wenn $e$ der Abstand des Berührungspunktes vom Scheibenmittelpunkt ist, so muB sie sich auf der Schraubenspindel, welche eine andere Winkelgeschwindigkeit besitzt, so lange verschieben, bis beide Geschwindigkeiten gleich sind. Ist etwa die Drehgeschwindigkeit der Schraube kleiner, so wird sich die Rolle nach dem Mittelpunkt der Scheibe hin verschieben. Wenn mit $\varrho$ der Halbmesser der Rolle bezeichnet wird, so wird ein Rubezustand eintreten, wenn $\varrho \omega_{2}=e \omega_{1}$ ist. Daher ist $e=\varrho \frac{\omega_{2}}{\omega_{1}}$ ein MaB für

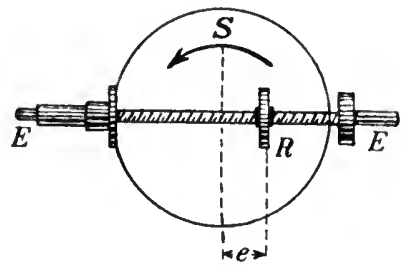

Fig. 27.

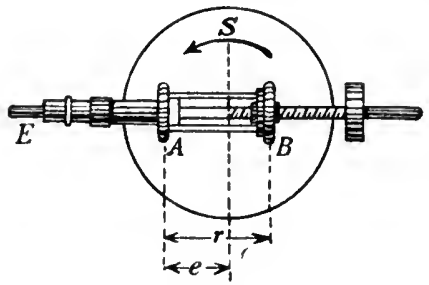

Fig. 28.

das Verhältnis beider Geschwindigkeiten, da ja @ eine Konstante ist. Da im allgemeinen die Geschwindigkeiten sich fortwïhrend ändern, so ist die augenblickliche Ablesung der Entfernung der Rolle vom Scheibenmittelpunkt das Verhältnis der gleichzeitig stattfindenden Geschwindigkeiten. Im Falle $\omega_{1}=1$ ist $e=\varrho \omega_{2}$ das $\mathrm{MaB}$ für die Geschwindigkeit der Schraubendrehung, die dann erhalten wird, wenn die Scheibe durch ein Uhrwerk in gleichförmige Umdrehung versetzt wird.

Die beschriebene Einrichtung wird besonders dann ungünstig, wenn $\omega_{1}$ kleine Werte annimmt, also $e$ sehr groß wird und die Rolle über den Rand der Scheibe sich entfernen würde. Diesem Übelstand hilft eine Veränderung (Figur 28) ab, die in der Anwendung zweier gleich großer Rollen $A$ und $B$ bestuht, die zwar auf derselben Achse sitzen und die konstante Entfernung $r$ des Halbmessers der Scheibe haben, aber sich unabhängig voneinander drehen. Erteilt man der Rolle $A$ mit dem Radius $\varrho$ die Geschwindigkeit $\omega_{1}$, so wird die Scheibe dadurch in eine Winkelgeschwindigkeit $\varphi$ versetzt, die sich bei einem Abstand $e$ der Rolle vom Mittelpunkte durch $e \varphi=\varrho \omega_{1}$ 
bestimmt. Die Rolle $B$, die den Abstand $r-e$ vom Mittelpunkte der Scheibe besitzt, wird dadurch die Geschwindigkeit $\varrho \omega_{2}=(r-e) \varphi$ erhalten. Wenn aber die ihr erteilte Geschwindigkeit $\omega_{2}^{\prime}$ nicht mit $\omega_{2}$ übereinstimmt, so wird eine Verschiebung der beiden Rollen, deren Abstand unveränderlich ist, so lange stattfinden, bis die Gleichheit $\omega_{z}=\omega_{2}^{\prime}$ erreicht ist. Dann ergibt sich

$$
\frac{d y}{d x}=\frac{\omega_{2}}{\omega_{1}}=\frac{r-e}{e} .
$$

31. Rollen- und Kugelmechanismus. Derselbe Gedanke liegt einem anderen Apparate zugrunde (Figur 29). Eine schräggestellte Scheibe $S$ wird durch Vermittlung eines Zahnrades $\boldsymbol{A}$ mit
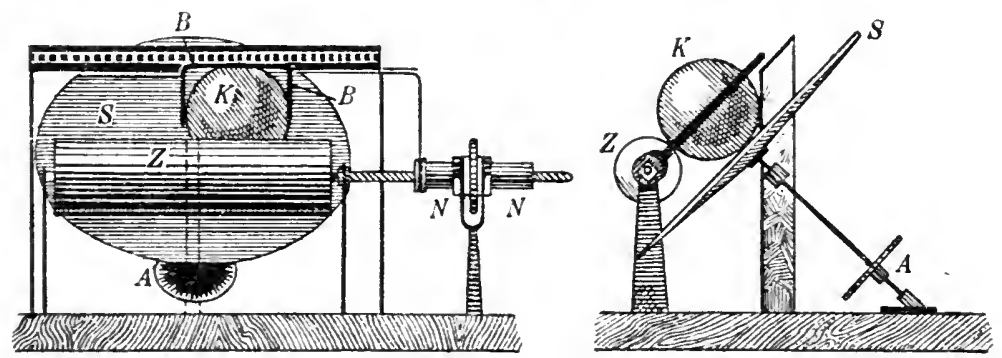

Fig. 29.

der Geschwindigkeit $\omega_{1}=\frac{d x}{d t}$ in Drehung versetzt. Längs eines horizontalen Durchmessers der Scheibe rollt eine Kugel $K$, die an dem Herabgleiten durch einen Zylinder $Z$ mit ebenfalls horizontaler Achse gehindert wird. Die Kugel berührt daher den ruhenden Zylínder längs einer Mantellinie, wenn sie sich längs des Durchmessers der Scheibe bewegt, während der Zylinder und die Scheibe nicht in Berührung stehen. Die seitliche Verschiebung der Kugel wird durch einen gabelförmig sie umfassenden Bügel $B$ geleitet, der an einer Schraubenmutter $N$ befestigt ist, die ohne sich zu drehen auf einer Schraubenspindel, welche die Verlängerung der Zylinderachse bildet, sich verschiebt. Die Winkelgeschwindigkeit, mit der sich die Schraubenspindel und damit der Zylinder dreht, sei $\omega_{2}=\frac{d y}{d t}$. Da nun die rotierende Scheibe der Kugel eine Drehgeschwindigkeit mitteilt, die vom Abstande des Berührungspunktes vom Scheibenmittelpunkt abhängt, und andererseits der Zylinder eine andere Drehgeschwindigkeit besitzt, so entsteht eine 
Verschiebung der Kugel, die so lange vorhält, bis beide Geschwindigkeiten, die der Kugel erteilt werden, gleich sind. Ein Zeiger, welcher an dem die Kugel umfassenden Bügel sich befindet, gestattet an einer Skala die Entfernung der Kugel vom Mittelpunkte abzulesen, die also das Verhältnis beider Geschwindigkeiten angibt.

Bereits im vorigen Abschnitt haben wir die Einrichtung erwähnt, bei der zwei rechtwinklig gegeneinander gerichtete Rollen auf derselben Kugel sich drehen und eine auf der Achse einer dieser Rollen verschiebbare Schraubenmutter mit der Kugelachse in Verbindung steht. Im Verfolg dieses Gedankens hat $\mathrm{Hel} \mathrm{e-S} \mathrm{haw}$ gezeigt, wie man auf diese Weise ebenfalls das Verhältnis der Geschwindigkeiten beider Rollen (nämlich $\frac{\omega_{2}}{\omega_{1}}=\operatorname{tg} \alpha$, s. S. 54) bestimmen und durch Anwendung von zwei Kugeln auch den zweiten Differentialquotienten, also die Beschleunigung erhalten kann. (Vgl. Philosophical Transactions vol. 176, Part. II, p. 367-402, London 1886.)

\section{Abschnitt.}

\section{Kurvenmesser.}

(Kurvimeter, Kurvometer, Kurveometer, Opisometer, Rektifizierrädchen, MeBrädchen, Longimeter, Linienmesser, Kartometer usw.)

32. Bei den folgenden Instrumenten wird die Summation von Größen, die graphisch gegeben sind, nach dem Verfahren der Integration ausgeführt. Die Integration bezieht sich entweder auf diese Größen selbst oder auf Funktionen derselben. Einer der einfachsten Fälle betrifft die Summierung der Bogenelemente einer Kurve oder der Bestimmung ihrer Bogenlänge. Da diese zwischen gegebenen Punkten gesucht wird, so vollzieht das Instrument eine Integration zwischen festen Grenzen, liefert also ein bestimmtes Integral. Der Einfachheit halber lassen wir die Bezeichnung der Grenzen der bestimmten Integrale in den folgenden Abschnitten fort.

Um die Bogenlängen einer Kurve zu messen, sind zwei Verfahren in Gebrauch. Das eine bedient sich einfach des Zirkels, mit dessen Hilfe Tangenten- oder Sehnenabschnitte gemessen und durch eine besondere Vorrichtung am Zirkel addiert werden. Die zweckmäßigste Zirkelöffnung richtet sich bei dieser Methode, die sich vorzugsweise auf Kurven von allmählicher Krümmungsänderung beschränkt, nach der Größe des Krümmungsradius. 
Die andere Methode der Rektifikation benützt als besonderes Instrument ein MeBrädchen, das gewöhnlich von der Hand geführt nach AugenmaB die Richtung der Tangente einhält und an einer Ablesevorrichtung für die Anzahl der Umdrehungen die Länge der Kurve bis auf einen vom Radius des Rädchens abhängigen Faktor in einem gewünschten Maße angibt. Dieses Verfahren ist also im Grunde dasselbe, welches der französische Arzt Fernel (1525) zum Zwecke einer Meridianmessung von Paris bis Amiens auwandte, als er die Umdrehungen seines Wagenrades zählte. Da wir einem solchen MeBrädchen bei verschiedenen Integrierinstrumenten begegnen werden, so führen wir $\varrho$ als Bezeichnung des Radius, $\omega$ als Bezeichnung des Winkels, den ein bestimmter Radius von einer Anfangslage ausgehend beschreibt, und $\sigma$ als Weg eines Punktes des Umfanges bei Drehung des Rades ein. Wenn das Rad mit einem hinreichenden Druck auf der Unterlage eine elementare Wegstrecke $d s$ durchläuft, so wird der von einem Punkte des Umfanges zurückgelegte Bogen $d \sigma=\rho d \omega$ und $d \sigma=d s$ sein. Bei Kurven muB die Ebene des Rades fortwährend ihre Richtung ändern. Bei wechselnder GröBe des Krümmungsradius der Kurve ist die GröBe des Radius $\varrho$ bald günstiger, bald ungünstiger. Durch Versuche ist festgestellt worden, da $B$ der Krümmungshalbmesser nicht wesentlich unter den Radhalbmesser herabsinken darf, wenn das Rädchen mit Erfolg benützt werden soll.

Das Kurveometer von Wittmann in Wien hat etwa $8 \mathrm{~mm}$ Durchmesser, das Rektifizierrädchen von Platzbecker ${ }^{1}$ ) in Düsseldorf etwa 6,5 mm. Bei der Befahrung einer geradlinigen Strecke wächst der unregelmäBige Fehler nahezu wie die Quadratwurzel der Länge, bei anderer Gestalt des Weges ist er natürlich gröBer. Hammer erbielt z. B. mit letzterem Instrumente bei einer geraden Wegstrecke von $500 \mathrm{~mm}$ einen unregelmäBigen Fehler $\pm 0,5 \mathrm{~mm}$, bei einem ungefähr gleichlangen willkürlich geformten Kurvenzug $\pm 0,9 \mathrm{~mm}$ als mittleren Fehler, wobei kein Krümmungsradius unter $10 \mathrm{~mm}$ vorkam. Erwähnt sei noch, daB bei dem Instrumente von E. KrauB in Mailand mit $4 \mathrm{~mm}$ Durchmesser das Zählwerk stets in derselben Richtung fortschreitet, auch wenn das Rädchen seinen Drehungssinn wechselt. ${ }^{2}$ )

1) Bei diesem Apparat wird besonders die Registriervorrichtung fïr Zählung der Radumdrehungen gerühmt.

2) Andere Instrumente, die auf demselben Prinzip berulen, sind 
Ein Linienmesser (Kurvometer) von G. Coradi in Zürich (Figur 30 ) ist mit 2 Rollen versehen, deren Achsen in derselben Richtung liegen und die unterhalb einer Platte mit 4 kreisförmigen Öffnungen angebracht sind. In zwei dieser Ausschnitte sind die Registrierscheiben der beiden Rollen $z z^{\prime}$ sichtbar. Genau in der Mitte zwischen den Auflagepunkten der beiden Rollenränder befindet sich der Führungspunkt, der längs der auszumessenden Linie verschoben wird, wobej die Richtung der Rollenachsen senkrecht zur augenblicklichen Tangente gestellt wird. Als Führungspunkt wird der Nullpunkt einer kleinen im mittleren Ausschnitte sichtbaren Skala $C$

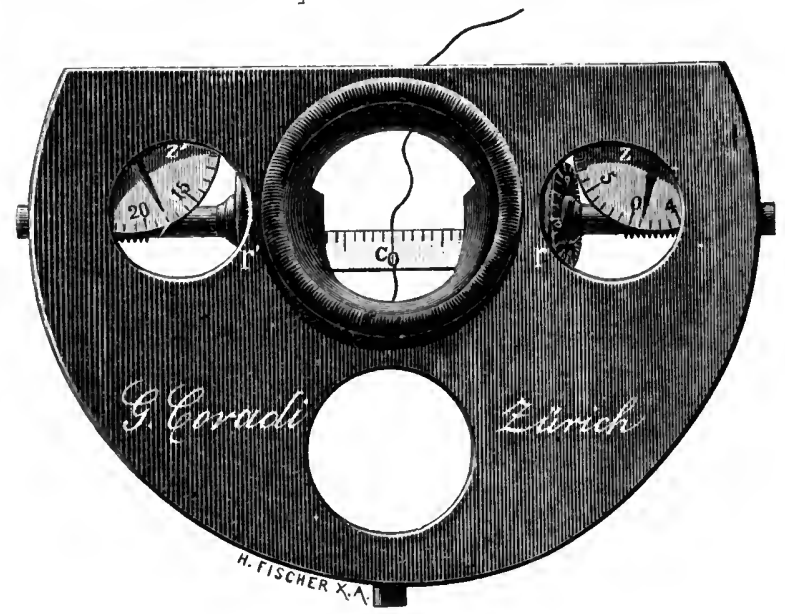

Fig. 30.

Denutzt. Die Bezifferung der beiden Registrierscheiben wächst im gleichen Sinne, so daß bei einer Drehung des Instrumentes um den Berührungspunkt die Summe der Ablesungen Null ist. Die Teilung ist so gewählt, $\mathrm{da} a$ die Summe der Ablesungen den zurückgelegten Weg angibt, wobei ein Teil einem Millimeter entspricht Der vierte Ausschnitt dient zum Anfassen des Instrumentchens. Die Genauigkeit wird vom Verfertiger auf $1 / 2000$ angegeben. Bei einer Abweichung um $8^{0}$ von der senkrechten Stellung der Skalenrichtung oder der Rollenachse zum Kurvenelement wird die Weglänge um $1 / 100$ unrichtig (entsprechend $\cos 8^{\circ}=0,99$ ).

von Schlagintweit, Jacob, Sand or, Mayer und Wolf in Fiume usf. konstruiert worden. 
Ein. Kurvenmesser von 0 tt in Kempten ${ }^{1}$ ) beruht in Kürze auf Folgendem: Ein Fabrstift trägt am unteren Ende einen Ring mit eingespanntem Hornblättchen, das zwei sich senkrecht kreuzende Linien enthält, deren eine in die Richtung der Kurventangente gedrebt wird. Am oberen Ende desselben Stiftes sitzt die Rolle, die durch Gegengewichte angedrückt auf der Unterseite eines darüber liegenden horizontalen Glasdaches rollt, das noch zur Vermeidung eines Gleiteus der Rolle mit Papier überzogen werden kann. Das Instrument wurde von $\mathrm{Hammer}$ auch bei starken Krümmungen, bis zu 0,8 mm Krümmungshalbmesser hinab, benutzt und gab bei Kurvenlängen von 120 bis $140 \mathrm{~mm}$ als mittleren Fehler $\pm 0,2$ bis $0,3 \mathrm{~mm}$ für eine einzelne Durchfahrung. Andere haben in der fortwährenden Drehung des Stiftes einen Übelstand geseheu.

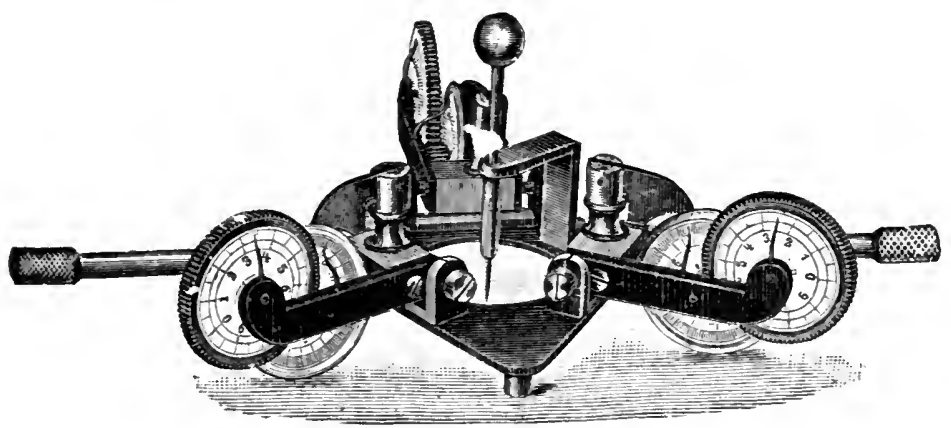

Fig. 31 .

Dieser Schwierigkeit begegnet das vom Ingenieur Fleischhauer in Gotha erfundene Kartometer (Konstrukteur Tesdorpf). Hier (Figur 31) befindet sich in der Mitte einer ringartigen Platte der Fahrstift, der durch zwei Führungsstangen regiert und auf der Kurve entlang geführt wird. GleichmäBig über den Umfang des Ringes sind eine ungerade Anzahl ( 3 in der Figur, auch 5 oder 7 ) Räder, deren Ebenen radial stehen, verteilt; die Räder, die sich infolge einer Sperrvorrichtung, die sich aber nicht bewähren soll, nur nach außen drehen können, sind mit Zifferblättern versehen, an denen die Umdrehungen und ihre Bruchteile abgelesen werden. Man bestimmt durch Umfahrung einer Kurve von genau bekannter

1) Abbildung Zeitschr. f. Verm. 18, 1889, S. 132.

Galle: Mathematische Instrumente. 
Länge, also etwa eines Kreisumfanges, die Konstante $k$, mit welcher die Summe der Ablesungsdifferenzen der Räder multipliziert werden muß und befährt die zu untersuchende Kurve zweimal, indem man jedesmal die Führungsstangen in derselben Richtung hält, beim zweiten Male aber senkrecht zu ihrer ersten Richtung stelit.

\section{Abschnitt.}

\section{Flächenmesser.}

(Instrumente zur Flächenbestimmung, Planimeter.)

33. Flächentafeln. Alle Instrumente, welche zur Ausmessung zweidimensionaler Größen dienen, verfolgen den Zweck, das Resultat durch eine einzige Zahl zu geben.

In der allereinfachsten, trotzdern aber für viele Zwecke durchaus geeigneten Weise wird dies durch die Quadratmillimeterglastafel erreicht, die auf die auszumessende Fläche gelegt wird. Die Anzahl der auf die Fläche fallenden Quadrate gibt unmittelbar den Inhalt an, während die an den Grenzen nur teilweise die Fläche deckenden Quadrate schätzungsweise addiert werden. Die Genauigkeit bewegt sich nach Lüdemann zwischen 0,05 und $0,1 \%$.

Etwas bequemer wird das Resultat mit einer Hyperbelglasta fel ${ }^{1}$ ) von Kloth in Osnabrück erhalten, bei der aber mehrere Verschiebungen nötig sind und einfachere Gestalten der Flächenstücke vorausgesetzt werden.

34. Harfenplanimeter und ähnliche. In demselben $\mathrm{Zu}$ -

1) Kloth stellte sich die Aufgabe, den geometrischen Ort der Schnittpunkte der stetig veränderlichen Koordinaten zu konstruieren, welche mit den rechtwinkligen Achsen ein Rechteck von konstanter Größe einschließen. $x y=F$ ist nun die Asymptotengleichung einer Hyperbel, und es sind auf der Glastafel für äquidistante Werte von $F^{\prime}$ die Hyperbeln ausgezogen. Bei einem Dreieck legt man die Grundlinie in die $y$-Achse, verschiebt dann die Tafel, bis die Spitze des Dreiecks mit der $x$-Achse zusammenfällt und liest am Erdpunkt der Grundlinie den Wert $F$ ab, der die doppelte Dreiecksfläche gibt. Ein Viereck zerlegt man durch eine Diagonale in 2 Dreiecke usf. Man kann auch die Eigenschaft der Hyperbeltangenten, daB sie Dreiecke von konstantem Flä̉cheninhalt mit den Koordinatenachsen einschlieBen zu einer veränderten Ablesung benutzen, (vgl. Zeitschr. f. Verm. 32, $369,1903)$. 
sammenhange ${ }^{1}$ ) ist das Harfenplanimeter von Oldendorp zu nennen. Es besteht in seiner ursprünglichen Gestalt aus parallelen, in einem rechteckigen Rahmen, in kleinen konstanten $\mathrm{Ab}$ ständen aufgespannten Fäden. In der Flächenformel $F=\Sigma y \Delta x$ werden die $y$ als die mittleren Längen der Flächenstücke zwischen je zwei im Abstande $\Delta x$ aufgespannten Fäden angesehen. Werden die Längen $y$ mit einem Zirkel (Planimeterzirkel, Planimeterhaarzirkel) gemessen, so ist es rorteilhaft, an diesem eine Additionsvorrichtung anzubringen (Zirkeladdition).

Da die Fäden nicht straff gespannt bleiben, ersetzt man sie durch eingeteiltes Ölpapier, das wiederum infolge nicht genügender Durchsichtigkeit die Konturen der Pläne nicht deutlich erkennen läBt. Deshalb wurde von Mönkemöller ein Planimeter ${ }^{2}$ ), das auf demselben Prinzip beruht, in folgender Weise konstruiert. Es besteht im wesentlichen aus einem riereckigen, eisernen Rahmen, in welchem eine Glasskala an einer Leitstange entlang verschoben werden kann. Die auszumessende Figur wird durch Teilstriche der Glasplatte in Parallelstreifen zerlegt, deren Breite konstant ist, und deren Längen durch die Verschiebung der Glasskala gemessen werden, die an einer Rolle abgelesen wird. Die Anwendung der Harfenplanimeter ist besonders bei langgestreckten Figuren empfehlenswert, die Genanigkeit des Mönkemöllerschen wird nach Untersuchungen von $\mathrm{Hamann}{ }^{3}$ ) derjenigen der später zu erwäbnenden Präzisionsplanimeter gleichgestellt. Beim Gebrauch muB darauf geachtet werden, daB nicht Flächenstreifen ausgelassen oder doppelt gemessen werden.

Auf wesentlich demselben Prinzipe beruht das ron F. Günther angegebene $\ddot{A} q u i-$ distanzplanimeter, welches zur Ausmessung von Detailfiguren (im MaBstabe 1:

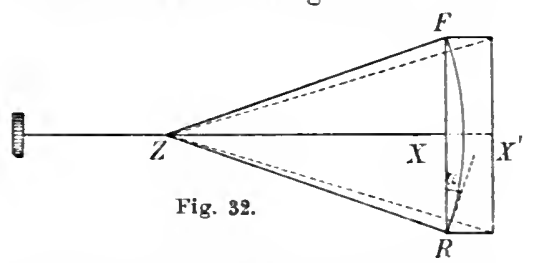
4000) konstruiert wurde. In der Hauptsache besteht es aus einer langen Schraube, deren Muttergewinde in einem auf einer festen Platte drebbaren Block eingelassen ist. An dem Ende, das dem

1) Ein ähnliches Hilfsmittel ist auch der Flächenmesser ron Semmler, Zeitschr. f. Verm. 35, 1906, 386, der aus einer fünfeckigen Glastafel mit anfgezeichneten Teilstrichen besteht.

2) Zeitschr. f. Instrk. 15, 456, 1895.

3) Zeitschr. f. Verm. 28, 549, 1899. 
Schraubengriff entgegengesetzt ist, befindet sich eine bewegliche Hülse, die zwei Arme trägt; der eine Arm trägt den Fahrstift, der andere die Zählrolle, die beide im gleichen Abstande von der Schraubenachse auf verschiedenen Seiten derselben einander gegenüberstehen. Setzt man den Fahrstift auf den dem Instrument nächsten Punkt des unteren Randes der auszumessenden Figur und dreht man den Schraubenarm um seinen Drehpunkt, so beschreibt der Fahrstift einen flachen Kreisbogen bis zum oberen Rand der Figur, wobei die Rolle sich abwälat. Jetzt schraubt man die Schraube um ein bestimmtes Stück (eine Viertelumdrehung) vorwärts und bringt den Fahrstift wieder an den unteren Rand der Figur. Hierbei wird durch eine Vorrichtung, die auch die Drehung der Schraube auf eine Vierteldrehung beschränkt, gleichzeitig die Rolle verhindert, sich zu drehen. Nun beschreibt der Fahrstift wieder einen Kreisbogen bis zum oberen Rande usf. und die Figur wird in eine Anzahl von Kreisringsektoren zerlegt.

Es sei (Fig. 32) $Z$ der Drehpunkt der Schraubenachse $Z X, F$ der Fahrstift, $R$ die Rolle, deren Mittelebene immer senkrecht zur. Schraubenachse steht, dann beschreibt, wenn $F$ einen Kreisbogen mit dem Radius $F Z$ beschreibt, $R$ einen kongruenten Kreisbogen und auch $X$ beschreibt einen Kreisbogen. Denn es ist $F X=X R$ und $F R$ senkrecht zu $X Z$, nach Konstruktion des Instrumentes, also $F Z=Z R$. Die Ebene der Rolle bildet nun mit der Tangente des Kreises einen kleinen Winkel $\alpha$, daher wickelt sie (S. 49) nicht den Bogen $\Delta s$ des Kreises $a b$, sondern $\Delta u=\Delta s \cdot \cos \alpha$. Durch die Drehung der Schraube erhält nun $Z X$ einen Zuwachs $X X^{\prime}=\Delta x$. Dadurch wird der Radius des Kreises, den der Fahrstift und der Rollenauflagepunkt beschreiben, um $\Delta r=\Delta x \cdot \cos \alpha$ vergröBert. Die Fläche eines Kreisringes beträgt daher

$$
\Delta s \cdot \Delta r=\Delta u \sec \alpha \cdot \Delta x \cos \alpha=\Delta u \cdot \Delta x .
$$

Durch passende Wahl der Ganghöhe der Schraube und geeignete Bezifferung der Rolle kann man, da $\Delta x$ eine Konstante ist, die Ablesung des Flächeninhalts unmittelbar an der Rolle erhalten.

Ein einfaches Hilfsmittel bietet der Planimeterschieber von R. A. de Wal $\left.{ }^{1}\right)$, der die Größe $(27 \mathrm{~cm})$ und Form eines gewöhnlichen Rechenschiebers (z. B. von Frank) besitzt. Er trägt

1) Zeitschr. f. Vermessungsw. 39, 111, 1910. Zeitschr. f. Instrumentenk. 31, 29, 1911. 
aber zwei gewöhnliche Teilungen von $2 \overline{\mathrm{j}} \mathrm{cm}$ Länge (den Naßstäben eines Planes in 1:2500 und 1:1000 entsprechend). Die Zunge trägt dieselben Teilungen, aber in entgegengesetzter Richtung laufend und nor mit Auszeichnung einiger Hauptstriche. Der Läufer ragt mit einer Glasplatte über den Stab hervor und trägt auf dieser und zwar senkrecht zu den Teilungen einen Indexstrich.

Legt man nun eine Harfe auf die auszumessende Figur, so kann man mit dem SchiebermaBstab, den man den Mittellinien der dadurch in parallele Streifen geteilten Figur parallel legt, die Streifen- (Mittellinien-)längen abmessen und additiv aneinandersetzen. Wenn die Länge des MaBstabes nicht mebr ausreicht, schiebt man die Zunge zum nächsten Strich und beginnt die Ylessung ron einem Zungenstrich am linken Ende des Maßstabes ausgehend. Als besonders rorteilhaft erscheint (nach Hammer) die gröBere Genauigkeit bei der Ausgleichung unregelmäBiger Streifenenden bei Anwendung des Indexstriches als durch AugenmaB.

35. Verwandlungsplanimeter. Für die Inhaltsberechnung geradlinig begrenzter Figuren (Polygone) kommen die Verwandlungsplanimeter in Betracht, wie sie Hofmann und Gangl off und Schlesinger konstruiert haben. Sie beruhen auf dem Prinzip des Parallelabschiebens zum Zwecke der Verwandlung des Polygons in ein flächengleiches Dreieck. ${ }^{1}$ )

Ein Lineal I aus Messing (Figur 33) besitzt längs einer Kante eine Einteilnng mit dem Null-

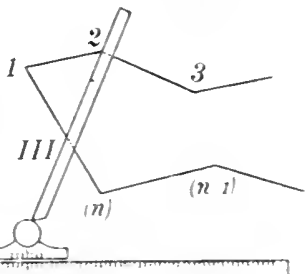
punkt in der Mitte. Ein kürzeres Lineal II läBt sich längs der geteilten Kante von I verschieben und hat eine Marke oder Noniusteilung, um die GröBe der Verschiebung messen zu können. An seiner anderen Längsseite ist ein drittes Lineal III durch ein Scharnier angebracht, um das es sich drehen kann. Die eine abgeschrägte Seite von III geht verlängert genau durch die Mitte des Scharniers und trägt eine Marke. Mit dieser wird es an einen Punkt (1) des Polygons angelegt, dann wird das Lineal I senkrecht zu III an II so gelegt, daB die Nullpunkte der Teilungen zusammenfallen und in dieser Lage mit zwei Nadeln fixiert. Das Verfahren

1) Vgl. Galle, Geodäsie. Sammlnng Schubert XXIII, 7. §4. 
ist dann weiter das folgende: Der bewegliche Arm III wird soweit gedreht, da $B$ er am Punkte (2) anliegt, darauf unter Beibehaltung der Richtung von III das Lineal II soweit verschoben, bis III wie-. der durch Punkt (1) geht. Jetzt wird der Arm III nach Punkt (3) gedreht, dann II mit III verschoben, bis III durch (2) geht usf. bis zuletzt der Arm III wieder an den Ausgangspunkt (1) angelegt und nach dem Punkte $(n)$ verschoben wird. Jetzt wird die Verschiebung $x$ gegen I an II abgelesen und man erhält, wenn die Entfernung der Marke auf III vom Umdrehungspunkte mit $h$ bezeichnet wird, den Flächeninhalt $F=\frac{1}{2} x h .^{1}$ )

Da die Parallelverschiebung des beweglichen Armes ohne Klemmvorrichtung schwierig ist, wird bei einer abgeänderten Konstruktion eine Glastafel auf die Zeichnung gelegt und der bewegliche Arm durch einen gespannten Faden ersetzt, der mit seiner Fassung auf der Glastafel aufliegt und daher bei Verschiebung der Tafel am Lineal I in seiner Richtung unverändert bleibt. Allerdings kommt hierbei eine Parallaxe bei der Ablesung in Frage, jedoch hat sich das Instrument bei Flächenberechnungen von Waldparzellen als nützlich erwiesen. ${ }^{2}$ )

36. Planimeter mit Integrierrolle. Bei dem Planimeter von Mönkemöller und dem Äquidistanzplanimeter sind Rollen zu dem Zwecke angebracht, die geradlinige bzw. die kreisförmige Bewegung durch die Umdrehungen der Rolle zu messen, doch mußte in beiden Fällen die Tätigkeit der Rolle bei der Rückwärtsbewegung ausgeschaltet werden. Bei der Verwendung einer Rolle als Hilfsmittel zur Integration fällt diese Beschränkung fort. Die Art der Verwendung bei den Planimeterkonstruktionen ist jedoch eine verschiedene. In erster Linie handelt es sich hierbei um eine Rolle mit flachem oder auch abgerundetem Rande, oder auch um einen Zylinder. Später kommen dann auch Planimeter mit scharfrandigen Rollen in Betracht. Aber nur die erste Art kann als Integrieroder Meßrolle bezeichnet werden, hierbei dient die Rolle dazu, die

1) Zum Beweise fällt man passender Weise von den beiden äußersten Polygonpunkten (deren einen man als (1) annehmen kann) Senkrechte auf die Richtung des Lincals I. Den Lagen von III vor und nach der jedesmaligen Verschiebung entsprechen zwei parailele Geraden. Die Entfernung des letzten Schnittpunktes von III und I vom Nullpunkt auf 1 ist dann die Grundlinie des Dreiecks, in das das Polygon verwandelt wird.

2) Bezüglich einiger praktischen Hinweise vgl. Zeitschr. f. Verm. $8,150,1879$. 
Verschiebungen eines Stabes zu messen, der die Fläche überstreicht, deren Inhalt bestimmt werden soll.

37. Theorie dieser Instrumente. Wir stellen uns zunächst einen Stab ron konstanter Länge $l$ rol und betrachten die ron ihm überstrichene Fläche. Wir nehmen an, daB der Stab durch kleine Verschiebungen nach und nach die Lagen $A_{0} B_{0}, A_{1} B_{1} \ldots$ $A_{n} B_{n}$ einnimmt. Um aus der Lage $A_{0} B_{0}$ in die benachbarte $A_{1} B_{1}$ zu gelangen, wird der Stab, wie wir uns vorstellen können, zunächst ein kleines Rechteck $A_{0} B_{0} B_{0}{ }^{\prime} A_{0}{ }^{\prime}$ überstreichen, indem seine Endpunkte die kleinen zu $A_{0} B_{0}$ senkrechten und gleichen Strecken $A_{0} A_{0}{ }^{x}$ und $B_{0} B_{0}{ }^{\prime}$ beschreiben, die dem $A$ bstand von $A_{1}$ ron der Geraden $A_{0} B_{0}$ gleich sind. Darauf wird er sich längs $A_{0}^{\prime} B_{0}{ }^{\prime}$ verschieben, bis sein Anfangspunkt nach $A_{1}$ gelangt. Durch eine Drehbewegung um $A_{1}$ als festen Punkt kommt dann sein Endpunkt nach $B_{1}$. In ähnlicher Weise wird die Bewegung ron $A_{1} B_{1}$ nach $A_{2} B_{z}$ in eine Parallelverschiebung, eine Längsbewegung und eine Sektorbewegung zerlegt usf. Je kleiner die Rechtecke und die Sektoren angenommen werden, um so mehr werden sich die Bewegungen der Endpunkte in kontinuierlichen Kurren rollziehen. Bezüglich des Vorzeichens setzen wir fest, daB einer Bewegung aus der Lage $A_{k} B_{k}$ nach $A_{k+1} B_{k+1}$ das positive Vorzeichen zukommt, wenn die neue Lage sich rechts von der alten befindet, das negative im andern Falle und da $B$ dementsprechend auch die überstrichenen Flächenteile mit dem positiven oder negativen Zeichen eingeführt werden. Auf diese Weise erhalten wir, wie dies notwendig ist, dasselbe Ergebnis, wenn wir statt $A_{0}$ nach $A_{0}^{\prime}$ und $B_{0}$ nach $B_{0}{ }^{\prime}$ zu verschieben, $A_{0}$ bis $A_{0}^{\prime \prime}$ und $B_{0}$ bis $B_{0}^{\prime \prime}$ ver-

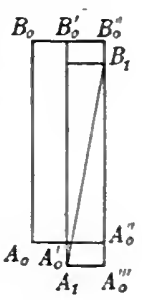

Fig. 34. rücken, wo $A_{0} A_{0}^{\prime \prime}=B_{0} B_{0}^{\prime \prime}$ der Abstand von $B_{1}$ yon $A_{0} B_{0}$ ist. Wenn wir dann $B_{0}{ }^{\prime \prime}$ nach $B_{1}$ in der Richtung $B_{0}{ }^{\prime \prime} A_{0}{ }^{\prime \prime}$ verschieben, so daB der Anfangspunkt nach $A_{0}^{\prime \prime \prime}$ kommt, so miissen wir dem Stabe eine negative Drehung erteilen, damit sein Anfangspunkt nach $A_{1}$ gelangt. Man sieht aus der Figur sofort, da die Sektoren flächengleich sind, da $B$ in beiden Fällen dieselbe ïberstrichene Fläche erhalten wird.

Bezeichnen wir die unendlich kleinen Parallelverschicbungen des Stabes mit $d s$, die unendlich kleinen Drehwinkel mit $d \varphi$, letztere in Bogenma $B$ gemessen, so ist der Inhalt der überstrichenen Fläche

$$
F=l \int d s+\frac{1}{2} l^{2} \int d \varphi .
$$


Die Grenzen der Integrale sind durch die Anfangs- und Endlage des Stabes bestimmt. Das erste Integral bezeichnet die Länge

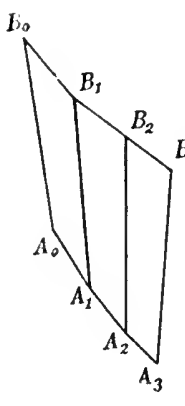

des Weges, den ein Punkt des Stabes senkrecht zur Stabrichtung zurücklegt (die Länge der orthogonalen Trajektorie sämtlicher Stablagen). Denken wir uns ferner (Fig. 35) durch einen beliebigen Punkt $P$ der Ebene Parallelen $\lambda$ zu sämtlichen Stabrichtungen gezogen, so ist $d \varphi=\left(\lambda_{k}, \lambda_{k+1}\right)$ der Winkel zwischen zwei aufeinander folgenden Richtungen. Das Integral $\int_{0}^{n} d \varphi$ zwischen der ersten und letzten ist daher der Winkel zwischen $\lambda_{0}$ und $\lambda_{n}$ und wir erhalten

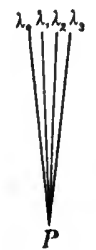

Fig. 35.

$$
F=l \int d s+\frac{1}{2} l^{2}\left(\lambda_{0}, \lambda_{n}\right)
$$

Wenn der Stab wieder in seine Anfangsrichtung zurückkehrt, so ist $\left(\lambda_{0}, \lambda_{n}\right)=0$ oder $=2 m \pi$, letzteres, wenn der Stab $m$ volle Umdrehungen gemacht hat. Ist aber $\left(\lambda_{0}, \lambda_{n}\right)=0$, so ist

$$
F=l \int d s .
$$

In den Figuren 36 und 37 sind solche Bewegungen angedeutet, bei denen die Endpunkte des Stabes die geschlossenen Figuren $A$ und $B$ durchlaufen. In der ersten Figur wird die zwischen $A$ und $B$ liegende Fläche $C$ zweimal überstrichen, einmal im positiven und einmal im negativen Sinne. Man erhält daher als Inhalt der ganzen vom Stabe überstrichenen Fläche bis zur Rückkehr in dieselbe Lage $F=A-B$. In der zweiten Figur ist die vom Stabe bestrichene Fläche $+(A-C)-(B-C)=A-B$, wenn wir den Flächen das ihnen zukommende Zeichen geben. Die kleinen überragenden Flächenstücke $D$ und $\boldsymbol{E}$ kommen nicht in Betracht, da jedes rom Stabe zweimal und zwar in entgegengesetzter Richtung überstrichen wird. Man kann sich auch die Figuren als Projektionen einer Art Röhrenflächen vorstellen, die von den den Stab darstellenden Geraden erzeugt werden. Die algebraische Summe der Projektionen aller Flächeninhalte einer geschlossenen Fläche auf eine Ebene ist nun Null. Die beiden Endflächen sind aber $A$ und $B$, die Mantelfläche ist $F$. Durch 
Projektion auf die Ebene $A$ z. B. erhalten wir $B+F=A$ oder wie oben $F=A-B$.

Zwingt man den einen Endpunkt des Stabes, z. B. $B$ auf einer Kurve von gegebenem Flächeninhalt (Kreis) oder dem Flächeninhalt Null (Gerade) sich zu berregen, so ist die vom andern Endpunkt $A$ umfahrene Fläche $A=F+B$ (wo $B$ im zweiten Fall null ist), wenn der Stab wieder in seine Anfangslage zurückkehrt.

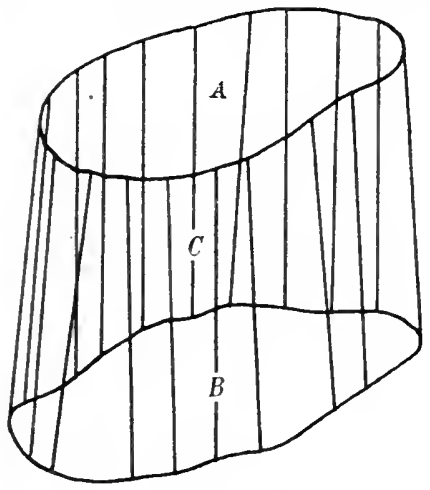

Fig. 36. Um also den Inhalt der von dem einen Endpunkt des Stabes um-

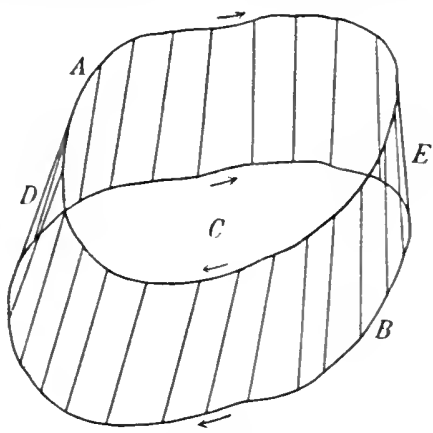

Fig. 37.

fahrenen Fläche zu bestimmen, ist außer der Kenntnis der Stablänge nur noch die Messung der Länge des Weges nötig, den ein Punkt des Stabes senkrecht zur Stabrichtung zurïcklegt.

Bevor wir die hierzu dienende Vorrichtung besprechen, möge noch eine analytische Beweisführung des der Planimetertheorie zu grunde liegenden Satzes folgen, wodurch seine Gültigkeit für alle Fälle dargetan wird. Wir nehmen ein rechtwinkliges Koordinatensystem an, bei dem die positive $y$-Achse der positiven $x$-Achse bei einer Bewegung im Sinne des Uhrzeigers folgt. Bei dem Stabe unterscheiden wir eine bestimmte Richtung vou $A$ nach $B$. Bei einer Drehung des Stabes um einen Endpunkt ist die überstrichene Fläche positiv, wenn die neue Richtung rechts von der alten liegt, also der Wiukel zwischen beiden im positiven Sinne rechts herumwächst. Haben wir z. B. ein Dreieck (Fig. 38), das

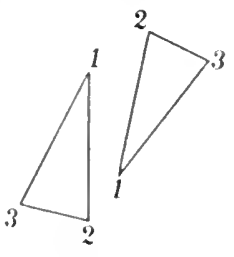

Fig. :8. von den beiden Stablagen $l_{1 \cdot 2}$ und $l_{1 \cdot 3}$ eingeschlossen wird, und sind $\varphi_{1 \cdot 2}$ und $\varphi_{1 \cdot 3}$ die Winkel derselben mit der $x$-Achse, so ist 
der Flächeninhalt des Dreiecks

$$
\begin{aligned}
& \frac{1}{2} l_{1 \cdot 2} \cdot l_{1 \cdot 3} \sin \left(\varphi_{1 \cdot 3}-\varphi_{1 \cdot 2}\right) \\
= & \frac{1}{2}\left[l_{1 \cdot 2} \cos \varphi_{1 \cdot 2} \cdot l_{1 \cdot 3} \sin \varphi_{1 \cdot 3}-l_{1 \cdot 2} \sin \varphi_{1 \cdot 2} l_{1 \cdot 3} \cos \varphi_{1 \cdot 3}\right] \\
= & \frac{1}{2}\left[\left(x_{2}-x_{1}\right)\left(y_{3}-y_{1}\right)-\left(x_{3}-x_{1}\right)\left(y_{2}-y_{1}\right)\right] \\
= & \frac{1}{2}\left[\left(x_{1} y_{2}-y_{1} x_{2}\right)+\left(x_{2} y_{3}-x_{3} y_{2}\right)+\left(x_{3} y_{1}-x_{1} y_{3}\right)\right],
\end{aligned}
$$

so dab eine zyklische Reihenfolge der Koordinaten der Dreieckspunkte rechts herum im Sinne des Uhrzeigers stattindet.

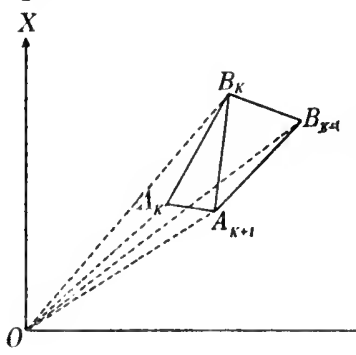

Fig. 39.

Nach diesen Vorbemerkungen betrachten wir zwei auf einander folgende, unendlich wenig verschiedene Stablagen $A_{k} B_{k}$ und $A_{k+1} B_{k+1}$ (Fig. 39). Die Koordinaten des Anfangspunktes $A$ bezeichnen wir mit $x, y$, diejenigen des Endpunktes $B$ mit $\xi, \eta$ und nach der Verschiebung die Koordinaten von $A_{k+1}$ mit $\rightarrow Y x+d x, y+d y$ und diejenigen von $B_{k+1}$ mit $\xi+d \xi, \eta+d \eta$. Für den Flächeninhalt $d f_{1}$ des Dreiecks $A_{k} B_{k} A_{k+1}$ erhalten wir dann

$$
\begin{aligned}
2 d f_{1}=(x \eta-y \xi)+\xi(y+d y)-\eta(x+d x)+(x+d x) y \\
\\
-(y+d y) x=(\xi-x) d y-(\eta-y) d x .
\end{aligned}
$$

Ebenso erhalten wir für den Inhalt $l f_{2} \operatorname{des}$ Dreiecks $A_{k+1} B_{k} B_{k+1}$ :

$$
\begin{aligned}
2 d f_{2}= & (x+d x) \eta-\xi(y+d y)+\xi(\eta+d \eta)-\eta(\xi+d \xi) \\
& \quad+(\xi+d \xi)(y+d y)-(\eta+d \eta)(x+d x) \\
= & \xi d \eta-\eta d \xi+y d \xi-x d \eta+(d \xi d y-d \eta d x) \\
= & (\xi-x) d \eta-(\eta-y) d \xi+\text { Glieder 2. Ordnung. }
\end{aligned}
$$

Nit Vernachlässigung der Glieder zweiter Ordnung in dem letzten Ausdruck folgt daher für die vom Stabe überstrichene Elementarfläche die Summe $d f_{1}+d f_{2}=$

$$
\left.d F=\frac{1}{2}(y d x-x d y)-\frac{1}{2}(\eta d \xi-\xi d \eta)+\frac{1}{2} d(\xi y-x \eta) \cdot{ }^{1}\right)
$$

1) Wir können die drẹi Teile auch geometrisch direkt ver- 
Die Integration ergibt zwischen den gegebenen Grenzen:

$$
F=\frac{1}{2} \int(y d x-x d y)-\frac{1}{2} \int(\eta d \xi-\xi d \eta)+\frac{1}{2}[\xi y-x \eta] .
$$

Das letzte Glied verschwindet, wenn der Stab in seine Anfangslage zurückkehrt. Das erste Integral stellt den Flächeninhalt der rom Anfangspunkt des Stabes, das zweite die rom Endpunkt

anschaulichen: Ist $O$ der Koordinatenanfang, so ist die Dreiecksfläche

$$
O A_{k} A_{k+1}=\frac{1}{2}[x(y+d y)-y(x+d x)]=\frac{1}{2}(x d y-y d x),
$$

die Fläche

$$
O B_{k} B_{k+1}=\frac{1}{2}\left[\xi(\eta+d \eta)-r_{i}(\xi+d \xi)\right]=\frac{1}{2}\left(\xi d \eta-\eta_{i} d \xi\right),
$$

endlich

$$
\begin{aligned}
& \begin{aligned}
& O A_{k+1} B_{k+1}-O A_{k} B_{k}=\frac{1}{2}[(x+d x)(\eta+d \eta)-(y+d y)(\xi+d \xi)] \\
&-\frac{1}{2}(x \eta-y \xi)=\frac{1}{2}\left(x d \eta+\eta d x-y d \xi-\xi d y+d x d \eta_{1}-d y d \xi\right)
\end{aligned} \\
& \text { und es ist } \quad=\frac{1}{2} d(x \eta-y \xi)+\text { Glieder 2. Ordnung }
\end{aligned}
$$

$$
\begin{aligned}
A_{k} B_{k} B_{k+1} A_{k+1} & =O B_{k} B_{k+1}-O A_{k} A_{k+1}+O A_{k} B_{k}-O A_{k+1} B_{k+1} \\
& =\frac{1}{2}(\xi d \eta-\eta d \xi)-\frac{1}{2}(x d y-y d x)+\frac{1}{2} d(y \xi-x \eta) .
\end{aligned}
$$

Auch als Summe eines Parallelogramms und eines Sektors läBt sich der Ausdruck auffassen. Es ist nämlich anders geschrieben:

$$
d F=\frac{1}{2}(\xi-x) d y-\frac{1}{2}(\eta-y) d x+\frac{1}{2}(\xi-x) d \eta-\frac{1}{2}(\eta-y) d \xi
$$

$=[(\xi-x) d y-(\eta-y) d x]+\frac{1}{2}[(\xi-x) d(\eta-y)-(\eta-y d(\xi-x)]$.

Der erste Teil ist die doppelte Dreiecksfäche $A_{k} B_{k} A_{k+1}$ oder die Fläche des Parallelogramms mit den Seiten $A_{k} B_{k}=l$ und $A_{k} A_{k+1}$. Der zweite Teil ist der Sektor mit dem Winkel $d \varphi$. Denn es ist

also

$$
\operatorname{tg} \varphi=\begin{aligned}
& \eta-y \\
& \xi-x
\end{aligned}
$$

und

$$
d \varphi=\frac{(\xi-x) d(\eta-y)-(\eta-y) d(\xi-x)}{(\xi-x)^{\xi}+(\eta-y)^{2}}
$$

daher

$$
(\xi-x)^{2}+(x-y)^{2}=l^{2},
$$

$$
\frac{1}{2} l^{2} d \varphi=\frac{1}{2}[(\xi-x) d(\eta-y)-(\eta-y) d(\xi-x)] .
$$

Bezeichnen wir noch den senkrechten Abstand der beiden Stablagen, welche den Seiten des Parallelogramms entsprechen, mit $d s$, so ist $d F=l d s+\frac{1}{2} l^{2} d \varphi$ und $F=l \int d s+\frac{1}{2} l^{2} \int d \varphi$ wie früher. 
umschriebene Fläche dar, so daB wie oben nach der früberen Bezeichnung $F=A-B$ ist.

Dasjenige Hilfsmittel, welches die Bewegungen eines Stabes senkrecht zu seiner Richtung miBt, ist die Rolle. Wir denken uns zunächst den Stab von der Länge $l$ als Achse einer in seiner Mitte angebrachten Rolle, wobei es nicht darauf ankommt, ob die Rolle am Stabe festsitzt, so $\mathrm{da} B$ er sich mit der Rolle dreht oder ob die Rolle frei beweglich ist. Die Rolle, die mit leisem Drucke auf die Unterlage gedrückt wird, kann man sich als ein fein gezahntes Rad vorstellen, und man sieht dann, daB bei schiefer Stellung der Radebene dieselbe Abwicklung stattfindet, wie wir oben bereits erwähnt haben. Hat die Rolle einen breiten Rand, wie eine Walze, so trägt sie in der Regel eine feine Riffelung, die man sich als enge Zahnleisten vorstellen wird, welche auf der Unterlage eine entsprechende Riffelung, wenn auch vorübergehend, hervorbringen. Bei schräger Riffelung wären die geradlinigen Zahnleisten durch Schraubenlinien (von großer Ganghöhe) ersetzt. Eine Schraube rollt aber auf ebener Grundlage ebenfalls senkrecht zu ihrer Achse, wenn nicht in der Achsenrichtung eine Kraft wirkt. Daher hat die Art der Riffelung keinen direkten Einfluß auf die Richtung der Rollenbewegung. ${ }^{1}$ )

Wird der Stab zwar parallel mit seiner Anfangsrichtung verschoben, beschreibt aber sein Mittelpunkt eine gegen die Stabrichtung geneigte Gerade, oder eine beliebige Kurve, so können wir uns die Bahn des Punktes aus unendlich kleinen geradlinigen Wegstrecken zusammengesetzt und diese in zwei Komponenten zerlegt denken, von denen die eine senkrecht, die andere parallel zur Stabrichtung liegt. Ist $\alpha$ der Winkel, den das Wegelement $d s$ mit der Richtung

1) Man kann sich am leichtesten diese Unabhängigkeit der Bewegung einer Walze oder Rolle von ibrer Riffelung dadurch veranschaulichen, daB man sich die einzelnen Riffelleisten in eine groBe Zahl getrennter Punkte aufgelöst denkt (wie auch in der Tat Walzen in dieser Weise bearbeitet werden, daB Erhebungen mit quadratischer Grundfläche stehen bleiben, um ihnen genügende Reibung zu verleihen). Dann ist es boi gleichmäBiger Verteilung dieser Punkte über die Oberfläche gleichgültig, ob man die in gerader Linie längs der Zylindererzeugenden stehenden sich verbunden denkt (Schraubenlinie von unendlich hoher Ganghöhe) oder ob man die durch eine mehr oder weniger steile Schraubenlinie verbundenen betrachtet. Da sich in der Literatur abweichende Ansichten finden, wurde die oben ausgesprochene auch experimentell erprobt. 
des Stabes oder der Rollenachse einschlieBt, so wird von der Rolle nur in der zum Stabe senkrechten Komponente das Stück $d \sigma=d s \cdot \sin \alpha$ abgewickelt, während bei der Bewegung in der Pichtung des Stabes der Berührungspunkt mit der Unterlage derselbe bleibt, also kein Rollen stattfindet. Dreht sich der Stab um seinen Mittelpunkt, so dreht sich die Rolle ebenfalls in demselben Auflagepunkte, so daB für die vom Stabe überstrichene Fläche sich der Inhalt null ergibt; in der Tat werden die beiden Sektoren von den Hälften des Stabes in entgegengesetztem Sinne überstrichen und ihre algebraische Summe ist daher null. Drebt sich der Stab um einen Punkt, der vom Rollenmittelpunkt den Abstand $c$ hat, um den Winkel $\psi$, so mißt die Rolle die Länge des von ihr beschriebenen Kreisbogens $c \cdot \psi$.

Es ist nun nicht notwendig, daB die Rolle sich im Mittelpunkt des Stabes befindet. Wir setzen nur roraus, daB der Stab sich parallel der Unterlage berregt. Es ist aber auch nicht erforderlich, daB die Rollenachse in der Richtung des Stabes liegt, sondern nur, daß sie dem Stab parallel und fest mit ihm verbunden ist. Für Parailelbewegungen des Stabes ist dies ohne weiteres ersichtlich. Wird aber der Stab aus der Richtung $O A$ um den Winkel $\varphi$ gedreht, so daB er in die Richtung $O A^{\prime}$ gelangt und ist $R$ die Lage der Rolle, die irgendwie fest mit dem Stabe verbunden ist, bei der Anfangsstellung des Stabes und $R^{\prime}$ für die Stellung $O A^{\prime}$, so fällen

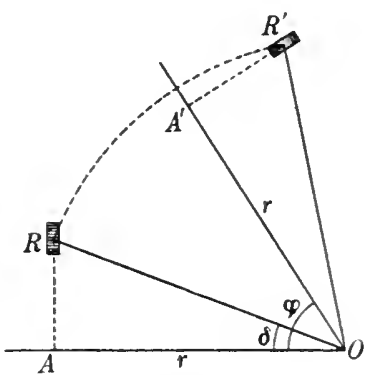

Fig. 40. wir (Fig. 40) die Senkrechten $R A$ und $R^{\prime} A^{\prime}$ auf die jedesmalige Stabrichtung und bezeichnen den Winkel $R O A=R^{\prime} O A^{\prime}=\delta$ und $O A=O A^{\prime}=r$. Da auch der Winkel zwischen der Rollenachse und $O R$ gleich $\delta$ ist, so beträgt die Abwicklung der Rolle für ein kleines Wegestück $d \sigma=d s \cdot \cos \delta$ oder da $d s=O R \cdot d \varphi=r \sec \delta \cdot d \varphi$ ist, $d \sigma=r d \varphi, d$. h. die Abwicklung ist dieselbe, als wenn die Rolle im Punkte $A$ des Stabes angebracht wäre.

38. Koordinatenplanimeter. Wenn wir in der Entwicklung der Formel für den Flächeninhalt auf S. 74, $75 x=\xi$ setzen, so findet eine Verschiebung des Stabes, der die Fläche überstreicht, parallel zur $y$-Achse statt und wir erhalten $d F=(y-\eta) d x$. Da nun in diesem Falle $y-\eta=l$ die Länge des Stabes darstellt, 
so wird bei der Rückkehr in dieselbe Anfangslage $F=0$. Es geht dies auch daraus hervor, daB die beiden Figuren $A$ und $B$ in diesem Falle kongruent sind, also $A-B=0$ wird. Wenn wir aber auf unsere allgemeine Betrachtung zurückgehen, so können wir uns einen unendlich langen Stab vorstellen, der sich um den unendlich fernen Punkt der $x$-Achse dreht und mit seinem andern Endpunkte die gegebene Fläche $A$ umfährt. Bei dieser Umfahrung wird also der Stab sich selbst und der $y$-Achse parallel bleiben und derjenige Teil der von ihm überstrichenen Fläche, der auBerhalb $A$ liegt, in entgegengesetztem Sinne zweimal überfahren werden, so daB man $F=A$ erhalten würde, während $B$, das durch einen unendlich fernen Punkt dargestellt wird, null ist. Statt des unendlich langen Stabes können wir daher auch einen endlichen Stab von veränderlicher Länge annehmen, indem wir ihn z. B. durch die $x$-Achse abgeschnitten denken. Wenn wir dementsprechend in unserer obigen Formel $\eta=0$ setzen, d. h. den einen Endpunkt der Geraden in die $x$-Achse verlegen. so kann darauf ein Planimeter begründet werden, das $F=\int y d x=A$ liefert. ${ }^{1}$ )

Der Gonella sche Integrationsmechanismus (s. S.51) dient zur Ermittlung eines solchen Integrals, wenn der Scheibe eine Drehung proportional $d x$ erteilt wird und die Rolle im Abstande $y$ vom Mittelpunkt der Scheibe durch ihre Verbindung mit dem Fahrstift gebalten wird.

Die erste Lösung dieser Aufgabe gab der Bergrat Josef Stadler zu Eisenerz in Steiermark durch das 1855 ersonnene und durch den Bergbeamten Smollin ausgeführte Hyperbel-

1) Um die Integration $\boldsymbol{F}=\int y d x$ anschaulich zu machen, zerlegen wir es im Falle einer auf derselben Seite oberhalb der $x$-Achse liegenden Fläche (und ähnlich in andern Fällen) in zwei Teile, indem wir den Fahrstift zunächst an der oberen Kontur im Sinne wachsender, also positiver $d x$ entlang führen, wobei $y$ mit $y_{1}$ bezeichnet werde. Die an dem Stabe angebrachte Rolle wickelt also die Betrïge $d x$ ab. Bei der Zurückführung des Fahrstiftes von seiner äußersten Stellung rechts an der unteren Kontur wird $-d x$ von der Rolle abgewickelt, und sind die Ordinaten hier $y=y_{2}$, so erhält man $y_{2} \cdot(-d x)$. Die Fläche der Figur ist nun gleich der znerst in der Richtung nach rechts überstrichenen Fläche minus der das zweite Mal überstrichenen Fläche innerhalb der Figur, also

$$
F=\int y_{1} d x-\int y_{2}(-d x)=\int y_{1} d x+\int y_{2} d x=\int y d x
$$


planimeter. Infolge der ungefähr gleichzeitigen Entstehung des A mslerschen Polarplanimeters, das einfacher und praktischer ist, hat es allerdings keine Verbreitung gefunden, seine Idee ist aber wert, kurz erläutert zu werden.

Ein Umdrehungkörper (Fig. 41) sei durch Rotation einer gleiehseitigen Hyperbel um eine ihrer Asymptoten entstanden, die die Richtung der $y$-Achse sei, und ein Zylinder, der auf ihm aufliegt, werde so verschoben, $\mathrm{da} B$ seine Achse der $x$-Achse parallel bleibt. Sein Berührungspunkt beschreibt bei dieser Bewegung eine Hyperbel, deren Gleichung $y z=k^{2}$ sei und $z$ ist der Radius eines Parallelkreises des Umdrehungskörpers, dessen Ebene senkrecht zur $y$-Achse steht. Der Einfachheit halber nehmen wir an, daß die Verlängerung der $\mathrm{Zy}$ linderachse den Fahrstift $F$ trägt, mit dem die auszumessende Figur umfahren wird. Bei einer Verrückung des Fahrstiftes parallel der $y$-Achse bleibt der Zylinder durch einen parallelogrammati-

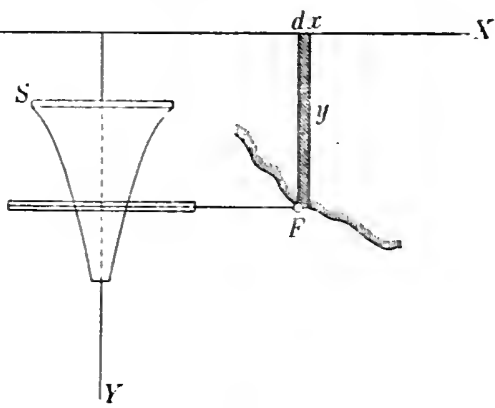

Fig. 41. schen Führungsmechanismus der $x$-Achse parallel und rollt von einem Parallelkreis zum anderen. Bei einer Bewegung $d x$ des Fahrstiftes parallel der $x$-Achse, wird der Zylinder in der Richtung seiner Achse rerschoben und bewirkt durch Reibung eine Drehung $d \varphi$ des Umdrehungskörpers um seine Achse. Es ist nun der unendlich kleine Kreisbogen $z d \varphi$ des Berührungskreises der Verschiebung des Zylinders $d x$ in seiner Achsenrichtung gleich, also $d x=z d \varphi$, mithin $k^{2} d \varphi=y d x$.

Die Umdrehungen des hyperbolischen Körpers und ihre Bruchteile werden durch eine Zählscheibe $S$ an seiner Grundfläche mittels eines Index abgelesen. Zeigte die Zählscheibe ror der Mressung die Ablesung $\varphi_{1}$, nach der Messung $\varphi_{2}$, so ist $k^{2}\left(\varphi_{9}-\varphi_{1}\right)=\int y d x=F$, also gibt die Ablesungsdifferenz bis auf eine von den Dimensionen des Umdrehungskörpers abhängige Konstante den Flächeninhalt der umfahrenen Figur. ${ }^{1}$ )

1) Die Konstruktion leidet an dem Mangel, daB der Zylinder bei seiner Parallelbewegung längs des Rotationskörpers nicht horizontal 
Gonella wandte bei seinem 1824 erfundenen Planimeter, das mit dem wenig beachteten des bayrischen Trigonometers Hermann (1814) Ähnlichkeit hat, eine Rolle an, die auf einer Kegelfläche läuft (Fig. 42). Er ließ zwei Räder in der geradlinigen Nut eines Lineals, das wir in der Richtung der $x$-Achse annehmen, hinter einander laufen. Das Gestell, welches sie verbindet, trug die Achse des drehbaren Kegels, der mit dem Grundkreis auf der Zeichenebene rollte, und zwar in der Weise, daß die jedesmal oberste Mantellinie des Kegels parallel der Zeichenebene war. Das Gestell, das man kurz als Wagen bezeichnen kann, wurde nun durch

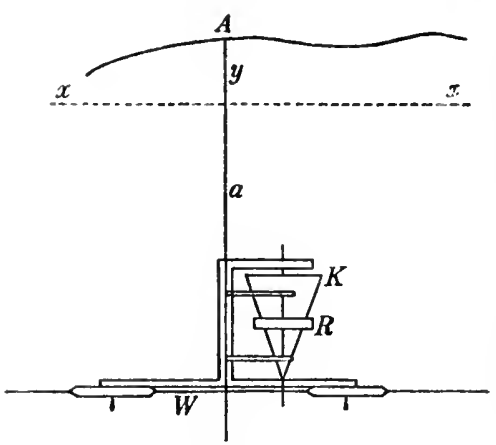

Fig. 42. eine Stange geführt, die senkrecht zum Lineale lag; an dieser Stange befindet sich der Fahrstift $F$, mit dem man die gegebene Figur umfährt. Führt man den Fahrstift um ein Stück $d x$ parallel zum Lineal, so verschiebt sich der Wagen um dasselbe Stück $d x$ und der Kegel dreht sich infolge der Reibung an der Unterlage um einen Winkel $d \varphi$. Wenn der Radius seines Grundkreises $k$ ist, so beträgt die Drehung $d \varphi=\frac{d x}{k}$. Auf dem Kegel liegt die

Integrierrolle auf, welche an der in ihrer Längsrichtung in einer Hülse verschiebbaren Führungsstange $a$ so befestigt ist, daß ihre Achse der Stange also der $y$-Achse parallel ist. Die Rolle berührt den Kegel in dem Abstaude $y$ von der Kegelspitze. Ist $h$ die Höhe des Kegels, so ist der Radius des Kreises, der durch den Berührungspunkt der Rolle senkrecht zur Kegelachse geht $r=\frac{y k}{\sqrt{k^{2}+h^{2}}}$. Die Abwicklung der Rolle bei der Drehung des Kegels ist daher $d \sigma=r d \varphi$ $=\frac{y k}{\sqrt{k^{*}+h^{2}}} d \varphi$. Bei einer Bewegung des Fahrstiftes parallel der $y$-Achse gleitet die Rolle, ohne sich zu drehen, längs der Kegelerzeugenden. Es findet also dabei keine Abwicklung statt. Jede beliebige Bewegung des Fahrstiftes kann nun als Resultante dieser

bleibt, so dab die Gleichung $d x=z d \varphi$ nicht streng erfüllt ist. Deshalb hat auch Stadler der Kurve auf Grund von Versuchen eine von der Hyperbel etwas abweichende Form gegeben. 
beiden Bewegungen verstanden werden; und man erhält daher, wenn $\sigma_{1}$ und $\sigma_{2}$ die Ablesungen der Rolle vor und nachher sind $\sigma_{2}-\sigma_{1}=\frac{1}{\sqrt{k^{2}+h^{2}}} \int y d x$ oder $F=\int y d x=\sqrt{k^{2}+h^{2}} \cdot\left(\sigma_{2}-\sigma_{1}\right)$.

Mit dem soeben beschriebenen stimmt auch das von Oppikofer, Ingenieur in Unteroppikon, 1827 in Verbindung mit dem Schweizer Mechaniker Ernst konstruierte Planimeter im Prinzipe überein, das in Paris 1836 miteinem Preise ausgezeichnet wurde.

Gonella selbst hat 1825 bei einem anderen Instrumente den Kegel durch eine kreisförmige Scheibe ersetzt, deren Drehung proportional derVerschiebung des Wagens ist. Ihm ist darin das Wetlischen Planineter ähnlich, das durch S tarke in Wien (1849) und Hansen in

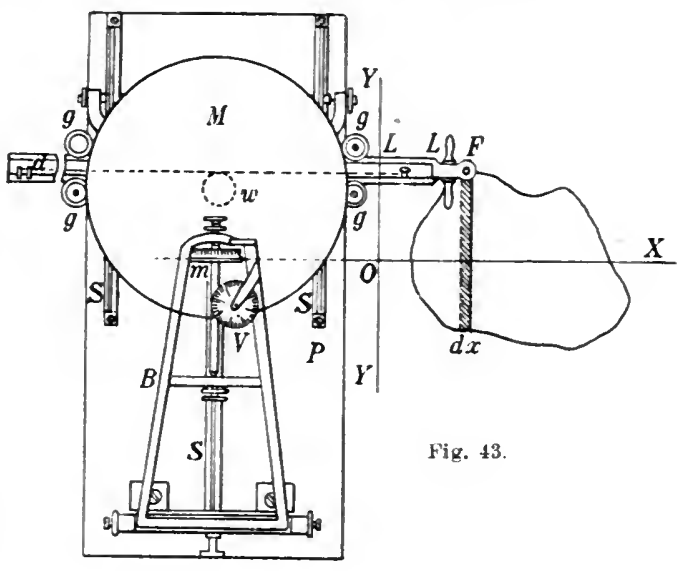
Gotha (1850) noch einige Änderungen und Verbesserungen erfuhr.

Das Wetli-Hansensche Planimeter (Fig. 43) ist auf einer starken Messingplatte $P$ aufgebaut. Ein dreifüBiges Gestell ähnlich dem eines Theodoliten läuft auf Rollen in drei parallelen Schienen $S$, wie ein Wagen, hin und her. In der Yitte des Dreifußes erhebt sich eine senkrechte Achse, um die sich, wieder ähnlich wie beim Theodoliten, eine horizontale Scheibe $M$ aus Messing dreht, deren Oberfläche ganz glatt mit Papier überzogen ist. Zwischen zwei Paaren von Führungsrollen $g$, die mit dem DreifuB in fester Verbindung stehen, läBt sich eine prismatische Stange $L$ senkrecht zur Schienenrichtung hin- und herschieben. Diese Stange trägt am Ende den senkrecht auf der Zeichenebene stehenden Fahrstift $F$, der also vermöge der Bewegung der Stange zwischen deu Führungsrollen in der einen Koordinatenrichtung (der $x$-Achse) sich bewegen läBt. Längs dieser Stange ist ein Silberdraht $d$ gespannt, der vermittelst einer unterhalb der Scheibe an ihrer Achse angebrachten Welle $w$, um die er sich schlingt, die Scheibe bei Ver- 
schiebung der Stange in ihrer Richtung in Umdrebung setzt. Nun ist ferner am schmalen Ende der Grundplatte ein senkrechter Ständer quer zur Schienenrichtung angebracht, der in Scharnieren einen aufklappbaren Rahmen $B$ trägt. Der Rahmen hält die Achse der MeBrolle $m$ in der Richtung des den Schienen parallelen Scheibendurchmessers. Bei der Bewegung des Wagens mit der Scheibe verschiebt sich dalier der Berührungspunkt der Rolle in diesem Durchmesser.

Ist $r$ die Summe der Halbmesser der Welle und des Drahtes, so bringt eine Verschiebung des Fahrstiftes um $d x$ durch den Silberdraht eine Drehung $d \varphi$ der Scheibe hervor, und es ist $r d \varphi=d x$. Ist ferner $y$ der Abstand des Auflagepunktes der an derselben Stelle bleibenden Rolle von dem Mittelpunkt der unter ihr sich verschiebenden Scheibe, so wird die Abwicklung der Rolle der Bewegung des Berührungspunktes auf der Scheibe gleich sein $y d \varphi=\rho d \omega=d \sigma$. Wenn man sich also durch den Rollenmittelpunkt die $x$-Achse gelegt denkt, so ist $y$ die Ordinate des Fahrstifts und $y d x=\rho r d \omega=r d \sigma$. Sind $\omega_{1}$ und $\omega_{2}$ die Ablesungen an der Rolle vor- und nachher, so ist $F=\int y d x=r \varrho\left(\omega_{2}-\omega_{1}\right)$, d. h. der Flächeninhalt $F$ ist der Differenz der Rollenablesungen bis auf einen von den Dimensionen der Rolle und der Welle abhängigen Faktor gleich.

Dieses Planimeter hat sich verhältnismäBig wenig Freunde erworben, obwohl es gerade bei kleinen Flächen mit einer 7 bis 8 mal größeren Genauigkeit als das später zu besprechende A m slersche Polarplanimeter arbeitet. Abgesehen von den höheren Anschaffungskosten ist dies darin begründet, daB die Leichtigkeit der Bewegung von ihrer Richtung abhängig ist. Auch ist es gegen äußere Einflüsse empfindlich. Dies gilt namentlich für den Draht, der leicht zu Störungen Veranlassung gibt.

Wir lassen auf dieses in der Geschichte der Planimetrie bekannteste ein neueres Koordinatenplanimeter folgen, das aus der Werkstatt von $\mathrm{Ch}$. Hamann in Berlin-Friedenau hervorgegangen ist.

Der ganze Apparat ist auf einer an den beiden Längskanten abgeschrägten Metallplatte montiert. Auf dieser ist an der einen kurzen Querseite der Pol $P$ (Fig. 44) festgemacht, um den sich $\operatorname{der} \operatorname{Arm} P A=r_{1}$ drehen läBt. Mit $P A$ hängt in $A$ durch ein Gelenk der ihm an Länge gleiche Arm $A B=r_{2}$ zusammen. Es ist nun dafür gesorgt, daß $B$ sich der Längskante parallel bewegt. Dies 
wird dadurch erreicht, da $B$ in einem Schlitten befestigt ist, der mit horizontal liegenden Rollen auf beiden Längskanten läuft. In $B$ befindet sich eine MeBrolle, deren Achse in die Richtung von $r_{z}$ fällt, deren Ebene also genau senkrecht zu $r_{2}$ steht. Durch Stellschrauben läBt sich die Gleichheit $r_{1}=r_{2}=r$ herstellen. Durch eine besondere Einiichtung der Rollenlager, die in einem Kreisringe liegen, wird eine Abweichung der Richtung der Rollenachse ron der Richtung $r_{2}$ korrigiert. Unter der Rolle hinweg ist ein flacher Fahrarm horizontal und senkrecht zu den Längskanten der Grundplatte verschiebbar angebracht. Auf seiner Oberseite ist eine Lage Papier eingelassen, auf der die Rolle mit einem geringen Drucke aufliegt, indem sie in einer Gabel innerhalb des erwähnten Ringes liegt. Auf dem Papier findet sie genügend Reibung und wird nicht abgenutzt. Es ist dafür gesorgt, daB der Fahrarm, der am Ende den Fahrstift trägt, senkrecht zu den Längskanten bei der Verschiebung des Schlittens bleibt, indem an diesem angebrachte federnde Rollen ihn an beiden Seiten führen.

Nehmen wir $P$ als Anfangspunkt rechtwinkliger Koordinaten, die Richtung $P B B^{\prime}$ als $y$-Achse, die dazu senkrechte Verschiebung des

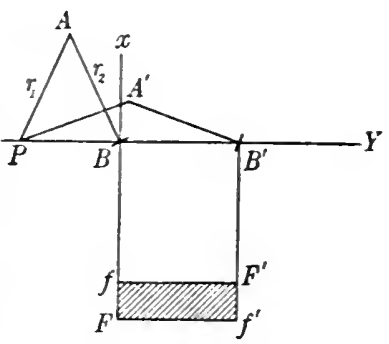

Fig. 44.

Fahrarms parallel der $x$-Achse an, so wird bei einer Bewegung des Fahrstifts $F f^{\prime}=d x$ die Rolle einen Bogen $d \sigma=\cos \alpha \cdot d x$ abwickeln. Bei einer Bewegung des Fahrstifts parallel der $y$-Achse ( $F f$ oder $f^{\prime} F^{\prime}$ ) bleibt der Berührungspunkt der Rolle mit dem Fahrarmlineal derselbe, es ändert sich nur der Winkel $\alpha$, den die Ebene der Rolle mit der $x$-Achse einschlieBt; dieser Winkel ist aber gleich dem Winkel an der Grundlinie des gleichschenkligen Dreiecks. Da $y=2 r \cos$ « für jede Lage von $B$ gilt, so erhält man

oder

$$
2 r \cos \alpha d x=2 r d \sigma
$$

mithin

$$
y d x=2 r d \sigma
$$

$$
F=\int y d x=2 r\left(\sigma_{2}-\sigma_{1}\right) .
$$

Wählt man die Teilung der Rolle so, daß ein Intervall doppelt so groB ist, wie in der Teilung auf dem Schenkel $r$, so erhält man 
den doppelten Wert der Rollenabwicklung $\sigma^{\prime}=2 \sigma$ durch direkte Ablesung, also

$$
F=r\left(\sigma_{2}^{\prime}-\sigma_{1}^{\prime}\right)=r \sigma^{\prime} .
$$

Die Länge $r$ kann zwar. am Schenkel $r_{2}$ abgelesen werden, indessen wird sie genauer durch Umfahren einer Figur von bekanntem Flächeninhalt erhalten. Zu diesem Zwecke wählt man etwa ein Quadrat auf Millimeterpapier oder benutzt ein sog. Kontrollineal. Das eine Ende eines solehen Lineals ist mit einer Nadelspitze versehen, die als Mittelpunkt des zu umfahrenden Kreises genommen wird. Der Fahrstift wird in eine der Vertiefungen eingesetzt, die das Lineal trägt, bei denen der Flächeninhalt des von ihnen beschriebenen Kreises angegeben ist. Ist $r_{0}$ der am Schenkel des Planimeters abgelesene Wert, so würde $F^{\prime}=r_{0} \sigma^{\prime}$ der genäherte Flächeninhalt sein. Wenn nun der mit dem Kontrollineal gefundene wahre Inhalt $F$ ist, so ist der Fehler $F-F^{\prime}=\left(r-r_{0}\right) \sigma^{\prime}$, woraus die wahre Schenkellänge

$$
r=\frac{F-F^{\prime}+r_{0} \sigma^{\prime}}{\sigma^{\prime}}
$$

folgt oder auch die Korrektion

$$
r-r_{0}=\frac{F-F^{\prime}}{\sigma^{\prime}} .
$$

Die beiden Schenkel $A P$ und $A B$ kann man zur Deckung bringen, indem man den Punkt $B$ unter den etwas höher angebrachten Pol $P$ schiebt. Auf diese Weise lassen sich durch Schräubchen die beiden Schenkel gleich lang machen.

Wir besprechen sogleich bei diesem Instrument die in ähnlicher Weise bei anderen vorkommenden Fehlerquellen. Es sind folgende Bedingungen vorhanden.

1. Die Rollenachse soll horizontal liegen. Bildet sie mit der Horizontalebene einen kleinen Winkel $\beta$, so wird (immer vorausgesetzt, daB sie in der Richtung $r_{2}$ liegt) die Wirkung nur die sein, da $B$ der Berührungspunkt $b$ ein wenig seitlich nach $b^{\prime}$ verschoben wird. Dies hat bei der Bewegung des Fahrarmlineals in der Richtung der $x$-Aclise unter der Rolle hinweg keinen EinfluB auf ihre Abwicklung, die beide Male gleich ist. Bei einer Verschiebung des Schlittens (Bewegung in der $y$-Achse) wird aber die Rolle nicht, wie es sein sollte, unbeweglich bleiben, da ja nur der senkrecht unter dem Schenkelende $B$ liegende Punkt eine ge- 
rade Linie beschreibt. Der Berührungspunkt wird also mit dem Radius $b b^{\prime}$ einen Kreisbogen beschreiben wodurch eine fehlerhafte Abwicklung entsteht. Bei vollständiger Umfahrung der Figur treten indes diese Abwicklungen beim Hin-und Rückweg des Schlittens mit entgegengesetzten Vorzeichen auf und heben sich im Ergebnis auf.

2. Die Rolle soll sich um ihren Mittelpunkt drehen. Ist aber die Rollenachse exzentrisch zur Rolle, der Drehpunkt $C^{\prime}$ um $C C^{\prime}=e$ vom Mittelpunkt $C$ entfernt (Fig. 45), so wird durch Drehung um den Winkel $\varphi$ statt des Bogens $O A$ der Bogen $O A^{\prime}$ abgewickelt. Das kleine Bogenstück $A A^{\prime}$ können wir mit dem Abstande $C^{\prime} D$ vertauschen und gleich $e \sin \varphi$ setzen. Wird die Rolle um $180^{\circ}$ gedreht, so beträgt der Febler der Rollenabwicklung $e \sin \left(180^{\circ}+\varphi\right)=-e \sin \varphi$. Man muB daher eine zweite Flächenmessung machen, bei der die Rollenablesungen um den halben Umfang verschoben sind, um

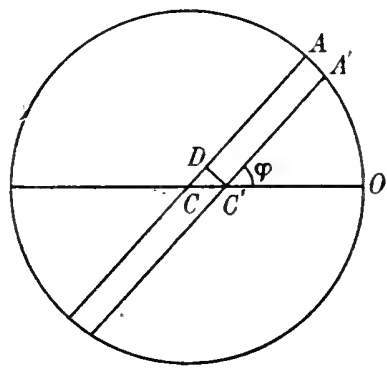

Fig. 45 . im Mittel der Umfahrungen das vom Exzentrizitätsfehler befreite Ergebnis zu erhalten.

3. Die Rollenachse soll die geradlinige Fortsetzung des Schenkels $A B$ (Fig. 44) bilden oder ihm wenigstens parallel sein. Ist aber ein kleiner Winkel $\psi$ zwischen beiden oder wie man sagt, Rollens chie fe vorhanden, so ist $d \sigma=\cos (\alpha+\psi) d x$. Da aber $y=2 r \cos \alpha$ bleibt, so wird $2 r d \sigma=$ $y d x-2 v \psi \sin \alpha d x$, wenn $\cos \psi=1$ und $\psi$ für $\sin \psi$ gesetzt wird. Man kann diesen Fehler beseitigen, indem man das gleichschenklige Dreieck auf die andere Seite der $y$-Achse verlegt und dann die Fläche nochmals umfahrt, denn dann wechselt $\psi$ sein Zeichen und das Mittel aus beiden Umfahrungen ist frei von Rollenschiefe. Das Durchschlagen geschieht bei dem Instrumente am einfachsten, wenn man beide Schenkel zur Deckung bringt

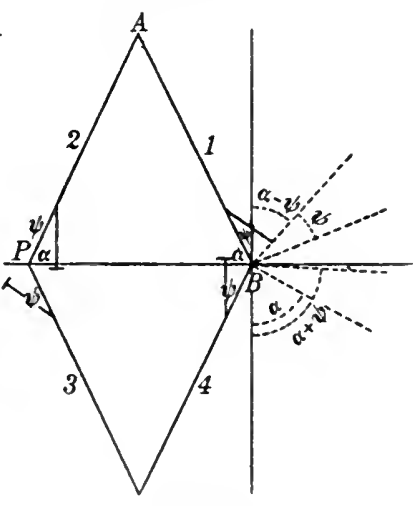

Fig. 46. und gleichzeitig nach unten dreht. ${ }^{1}$ ) Will man aber nur in einer

1) Um dies leichter zu überblicken, sind in Figur 46 die vier 
Lage arbeiten, so muß man die Rollenschiefe durch die Korrektionsschrauben beseitigen.

4. Die beiden Hauptbewegungsrichtungen des Instrumentes sollen senkrecht gegen einander sein. Weicht aber die Richtung des Fahrarms von der Senkrechten auf den Längskanten der Grundplatte um einen kleinen Winkel $z$ ab, dann wird der Fahrstift die Strecke sec $z d x$ statt $d x$ zurücklegen. Da die Rolle zugleich den Winkel $\alpha-z$ statt $\alpha$ mit dem Fahrarm bildet, so erhält man

$$
d \sigma=\cos (\alpha-z) \sec z d x=\cos \alpha d x+\sin \alpha \operatorname{tg} z d x
$$

mithin

$$
2 r d \sigma=2 r \cos \alpha d x+2 r \sin \alpha \operatorname{tg} z d x=y d x+2 r \sin \alpha \operatorname{tg} z d x .
$$

Wird die Figur mit durchgeschlagenen Schenkeln durchfahren, so tritt $-z$ an Stelle von $+z$, daher ist das Mittel aus beiden Umfahrungen von diesem Fehler befreit.

5. Die Achsen der Scharniere sollen senkrecht zur Zeichenebene stehen. Dies gilt sowohl für das Polgelenk $P$, wie für das Gelenk im Punkte $\boldsymbol{A}$. Ist letzteres nicht der Fall, so beschreibt der Endpunkt des Armes $A B$ um den Endpunkt des Armes $P A$ einen Kreisbogen, das Scharnier selbst eine Kegelfläche. Dieser Fehler (Scharnierschiefe), der vielfach überschätzt worden ist, läßt sich nicht eliminieren.

Die Genauigkeit des Instrumentes, das besonders deshalb nicht für große Figuren geeignet ist, weil bei lang herausgezogenem Fahrarm die Sicherheit seiner Führung senkrecht zur $y$-Achse verloren geht, beträgt etwa $1 / 300$ bis $1 / 350$ des Flächeninhaltes, die bei kleinen Figuren bis auf etwa $1 / 200$ herabsinkt. Es hat denselben Nachteil, wie das Wetlische Planimeter, daB in den beiden Hauptrichtungen die Bewegung spielend vor sich geht, dagegen in anderen Richtungen je nach ihrer Lage ein gröBerer oder geringerer Widerstand überwunden werden muB.

Lagen des Schenkels $A B$ unterschieden, wobei die Rollenschiefe stark ïbertrieben eingezeichnet ist. Lage 1 deutet die Stellung bei der ersten Messung an, bei 2 ist der Schenkel $A B$ mit $A P$ zusammengeklappt, bei 3 ist die Drehung beider Schenkel vollzogen, bei 4 ist $A B$ wieder von $A P$ entfernt und hat die der Lage 1 entsprechende Stellung zur zweiten Messung. Der Winkel der Rollenebene mit der $x$-Achse ist bei Lage $1: \alpha-\psi$, bei Lage $4: \alpha+\psi$. 
Wir deuten noch ein anderes Prinzip an, auf dem das 1852 von E. Sang beschriebene Planimeter beruht (Fig. 47). Ein Doppelkegel rollt mit seinen beiden gleichen Grundkreisen in einer der $x$-Achse parallelen Richtung. Die Registrierrolle $R$ ist derartig angebracht, $\mathrm{daB}$ sie einen Kreis des Kegels berührt, der den Abstand $y$ von der in der $x$-Achse sich bewegenden Kegelspitze hat. Den Fahrstift denken wir uns so mit der Rolle verbunden, daB er denselben Abstand $y$, wie die Rolle, ron der $x$-Achse hat. Ist $a$ der Radius der Grundkreise, so wird bei einer Bewegung des Fahrstiftes und des Kegels um $d x$ der Kegel eine Drehung $d \varphi$ um seine Achse erfahren, die durch $d x=a d \varphi$ bestimmt ist. Ein Punkt des Berührungskreises mit dem Radius $r$ wird, wenn $h$ die Höhe der Kegel

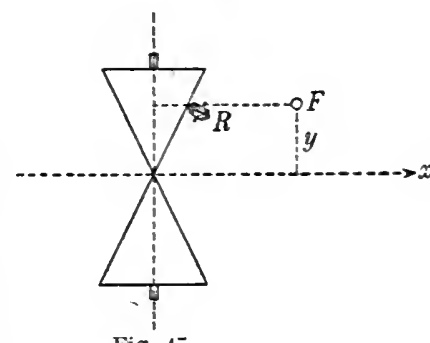

Fig. 4 . ist, den Bogen $r d \varphi=\frac{1}{h} y d x$ beschreiben, der durch die Rolle gemessen wird (vgl. S. 80). Es ist also

und

$$
h d \sigma=y d x
$$

$$
h\left(\sigma_{2}-\sigma_{1}\right)=\int y d x=F .
$$

39. Linearplanimeter. In der allgemeinen Flächenformel (S. 73, 75) $F=A-B$ kann das zweite Integral

$$
B=\frac{1}{2} \int^{2}(\eta d \xi-\xi d \eta)
$$

auch dadurch zum Verschwinden gebracht werden, da $B$ wir

$$
\frac{\eta}{\xi}=c
$$

(konstant) setzen; dies bedeutet, daB der eine Endpunkt $(\xi, \eta)$ des die Flăche überstreichenden Stabes auf einer durch den Koordinatenanfang gehenden Geraden zu laufen gezwungen wird. Diese Gerade kann aber auch ganz beliebig liegen; denn setzen wir

so folgt

$$
\frac{\eta-b}{\xi-a}=c,
$$

$$
(\eta d \xi-\xi d \eta)-(b d \xi-a d \eta)=0 \text {. }
$$


Bei der Integration fallen die beiden Integrale

$$
b \int d \xi \text { und } a \int d \eta
$$

fort, wenn wir die Fläche vollständig umfahren, also die Grenzen für den Anfangs- und Endpunkt dieselben sind. Wir bezeichnen solche Planimeter, bei denen der eine Stabendpunkt in einer Geraden sich hin und her bewegt, als Linearplanimeter.

Hierher gehört das von dem Erfinder des Hyperbelplanimeters (S. 79) Stadler ersonnene und von ihm Rollplanimeter be-

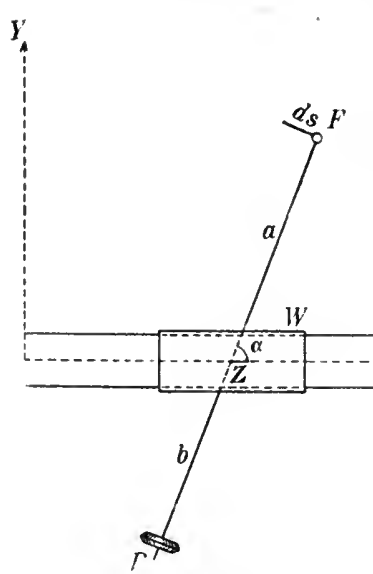

Fig. 48. nannte Instrument. Auf zwei Schienen $S S$ gleitet ein Wagen $W$, der den um den Zapfen $Z$ drehbaren Fahrarm $F R$ trägt (Fig. 48). An dem einen Ende $F$ ist der Eahrstift, am andern $R$ die Rolle befestigt, deren Achse mit der Richtung des Fahrarms zusammenfällt. Sei die $x$-Achse parallel, die $y$-Achse senkrecht zu den Schienen, so mögen $x, y$ die Koordinaten des Fahrstiftes $F$, $x^{\prime}, 0$ die des Drehpunktes $Z$ und $X, Y$ die des Rollenmittelpunktes bzw. des senkrecht unter ibm liegenden Auflagepunktes bezeichnen. Ferner sei

$$
F Z=a, \quad R Z=b
$$

und $\alpha$ der Winkel den $Z F$ mit der $x$-Achse bildet. Es ist dann

$$
x=x^{\prime}+a \cos \alpha, \quad y=a \sin \alpha .
$$

Beschreibt der Fahrstift den kleinen Weg

$$
d s=\sqrt{d x^{2}+d y^{2}},
$$

so sind seine Komponenten

$$
d x=d x^{\prime}-a \sin \alpha d \alpha, \quad d y=a \cos \alpha d \alpha .
$$

Die Rolle macht gleichzeitig einen Weg $d S$, der sich aus einer Kreisbewegung um $Z$ mit dem Radius $b$ und einer Parallelbewegung zur $x$-Achse zusammensetzt. Die Rollenabwicklung gibt nur den zur Rollenachse senkrechten Teil dieser Bewegungen 


$$
d \sigma=b d \alpha+\cos \left(270^{\circ}+\alpha\right) d x^{\prime}=b d \alpha+\sin \alpha d x^{\prime}
$$

oder

$d \sigma=b d \alpha+\sin \alpha d x+a \sin ^{2} \alpha d \alpha=\frac{1}{a} y d x+\left(b+a \sin ^{2} \alpha\right) d \alpha$ und

$\sigma_{2}-\sigma_{1}=\frac{1}{a} \int y d x+\left(b+\frac{a}{2}\right)\left(\alpha_{2}-\alpha_{1}\right)-\frac{a}{4}\left(\sin 2 \alpha_{2}-\sin 2 \alpha_{1}\right)$.

Wenn man die Figur $^{1}$ ) vollständig umfährt, also zur Anfangslage $\alpha_{2}=\alpha_{1}$ zurückkehrt, so erhält man einfach:

$$
a\left(\sigma_{z}-\sigma_{1}\right)=\int y d x=F .
$$

Da dieses Resultat die Rollenarmlänge $b$ überhaupt nicht mehr enthält, so kann man $b$ auch zu Null machen, und man gelangt zu dem Linearplanimeter von A. Miller Ritter von Hauenfels (1860 ron Breithaupt \& Sohn in Kassel angefertigt). Ein Lineal von $52 \mathrm{~cm}$ Länge bildet die Führungsbahn für einen Wagen mit zwei Rollenpaaren, von denen das eine in einer Nut von halbkreisförmigem Querschnitt, die beiden andern Rädchen mit etwas breiterem Kranze auf der Ebene des Lineals rollen. $\left.{ }^{2}\right)$ Die Fahrstange, welche sich an einer Stelle zu einem Ring erweitert, wird mit diesem in eine kreisförmige Öffnung des Wagens horizontal eingelegt. Um eine zum Lineal senkrechte Achse in Nittelpunkt des Ringes bzw. der kreisförmigen Öffnung läBt sich die Fahrstange drehen. In derselben rertikalen Achse liegt der Berührungspunkt der MeBrolle, welche bei Bewegung des Fahrarms und des Wagens sich auf der mit Papier überzogenen Gleitbahn des Lineals abwälzt, und deren Teilung ein Nonius gegenübersteht. Die horizontale Drehachse der Rolle läuft in Spitzenschrauben und trägt eine Schraube ohne Ende, welche mit einem im Fahrarm eingelassenen Zählwerk in Verbindung steht. Die Länge des Fahrarms kann verändert werden; zur Ausgleichung seines Gewichtes ist an seiner Rückwärtsverlängerung ein Gegengewicht angebracht. Neben dem Fahrstifte sind zwei Stützen angebracht, die mit dem Auflagepunkt der Rolle zusammen die wagerechte Lage des Fahrarms gewährleisten. Die Theorie ist dieselbe wie beim vorigen Instrument, wenn man $b=0$ setzt. Auch kann man

1) Das Planimeter muB so aufgestellt werden, daB die auszumessende Fläche ganz umfahren werden kann.

2) Om Spannungen zu vermeiden. 
passend $a=a_{0}+m i$ setzen, wenn die Fahrarmlänge um $m$ Teile von der Länge $i$ auf der Skala des Fahrarms verändert wird. Bei diesem Instrument kann auch der Fall vorkommen, daB das Lineal innerhalb der zu bestimmenden Fläche liegt und infolgedessen der Fahrarm eine volle Umdrehung macht, um in seine ursprüngliche Lage zurückzukehren. Dann erhält man

$$
\begin{aligned}
F & =a\left(\sigma_{2}-\sigma_{1}\right)-a^{2} \int_{\alpha_{1}}^{\alpha_{1}+2 \pi} \sin ^{2} \alpha d \alpha \\
& =a\left(\sigma_{2}-\sigma_{1}\right)-a^{2}\left[\frac{\alpha}{2}\right]_{\alpha_{1}}^{\alpha_{1}+2 \pi}+a^{2}\left[\frac{\sin 2 \alpha}{4}\right]_{\alpha_{1}}^{\alpha_{1}+2 \pi}
\end{aligned}
$$

oder

$$
F=\left(a_{0}+m i\right)\left(\sigma_{2}-\sigma_{1}\right)-a^{2} \pi,
$$

d. h. es tritt noch die Fläche eines Kreises mit der Fahrarmlänge als Radius hinzu.

Die Konstruktion eines Linearplanimeters von Amsler (1882) ist im Prinzip ähnlich (Fig.49). Auch hier läuft ein kleiner Wagen in den Nuten eines Lineals. Dieser trägt eine horizontale runde Scheibe, die bei der Bewegung des Wagens vermittels einer Übertragung um ihre vertikale Achse gedreht wird, so daß die Drehung der Verschiebung des Wagens proportional ist. Auf dieser mit Papier überzogenen Scheibe liegt die mit dem Fahrarm in fester Verbindung stehende Integrierrolle auf, deren horizontale Achse je nach der Stellung des um einen Punkt des Wagens drehbaren Fahrarms verschiedene Winkel mit dem durch ihren Berührungspunkt gehenden Scheibenradius einschlieBt.

Bei diesem und dem vorhergehenden Planimeter ist die $\mathrm{Ab}$ wicklung der Integrierrolle unabhängig von der Beschaffenheit und den Unebenheiten der Zeichenfläche. Das Amslersche Instrument ist nur zur Umfahrung langgestreckter, in der Richtung des Lineals sich ausdebnender Figuren geeignet, da die Drehbewegung des Fahrarms eine beschränkte ist, wenn die Rolle auf der Scheibe bleiben soll.

Da die Drehung der Scheibe der Fortbewegung des Wagens proportional ist, kann man das Element des Drehungswinkels $d \varphi=p d x_{0}$ setzen, wenn $x_{0}$ die Abszisse des Drehpunktes 0 ist. Wenn $\beta$ der Winkel der Rollebene mit dem Scheibenradius $r$ ist, so ist die Rollenabwicklung $d \sigma=\sin \beta r d \varphi=p r \sin \beta d x_{0}$. Denn nur die Bewegung des Wagens, nicht aber die Drehung des Fahr- 
arms, bei der die Rolle in der Richtung ihrer Achse verschoben wird, hat eine Abwicklung zur Folge. Wird die unveränderliche Entfernung des Scheibenmittelpunktes rom Drehpunkt des Fabrarms $O S=s$ gesetzt, so ist

also

$$
r \sin \beta=s \sin \alpha,
$$

$$
d \sigma=p s \sin \alpha d x_{0} .
$$

Nun ist wie früher

$$
d F=a \sin \alpha d x_{0}-a^{2} \sin ^{2} \alpha d \alpha,
$$

mithin

und

$$
d F=\frac{a}{p s} d \sigma-a^{2} \sin ^{2} \alpha d \alpha
$$

$F=\frac{a}{p s}\left(\sigma_{\Xi}-\sigma_{1}\right)-a^{2} \int \sin ^{2} \alpha d \alpha$,

wo das zweite Glied bei der Rückkehr zur Anfangslage Null wird (bzw. $=\pi a^{2}$, wenn der Fahrarm eine volle Umdrehung gemacht hat).

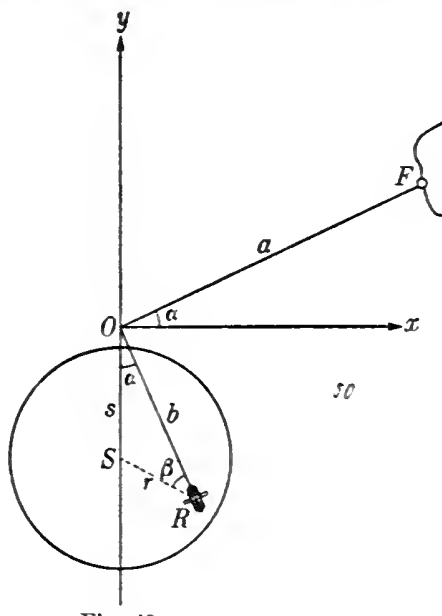

Fig. 49.

Man kann die besprochenen Linearplanimeter als Vorläufer des Rollplanimeters rou Hohmann-Coradi ansehen, das ebenfalls besonders für langgestreckte Figuren (und zwar ron beliebiger Länge) sich eignet (Fig. 50). Bei ihm bewegt sich ein Gestell auf zwei an den Enden einer Längsachse sitzenden breiten Walzen in der zu ihr senkrechten Richtung. Auch hier trägt das Gestell eine horizontale Scheibe, die sich bei der Fortbewegung des Instrumentes proportional dem zurückgelegten Wege um ihre senkrecht stehende Achse dreht. Der Fahrarm läBt sich um eine ebenfalls an dem Gestell angebrachte senkrechte Achse drehen und ist im rechten Winkel mit dem Rollenarm verbunden, der die auf der Scheibe aufliegende Integrierrolle trägt, deren Achse der Richtung des Fahrarms parallel ist.

Es seien $W_{1}$ und $W_{2}$ die beiden walzenförmigen Rollen, die auf besondere Art rauh gemacht, die Parallelbewegung des Instrumentes in der Richtung der $x$-Achse gewährleisten; die eine ron ihnen, $W_{1}$, ist an einer Seite mit einem konischen, gezahnten Rädchen rersehen, das in ein auf der Achse der Scheibe $S$ sitzendes horizontales Rädchen eingreift. Ist $R_{1}$ der Radius der Walze $W_{1}$ 
(und $W_{2}$ ), $R_{1} d \psi=d x_{0}$ ihre Abwicklung (gleich dem Fortrücken des Drehpunktes $O$ des Fahrarms in der Richtung der $x$-Achse), so dreht sich das mit ihr verbundene konische Zahnrad, das an der Berührungsstelle mit dem Scheibenrad den Radius $R_{2}$ haben

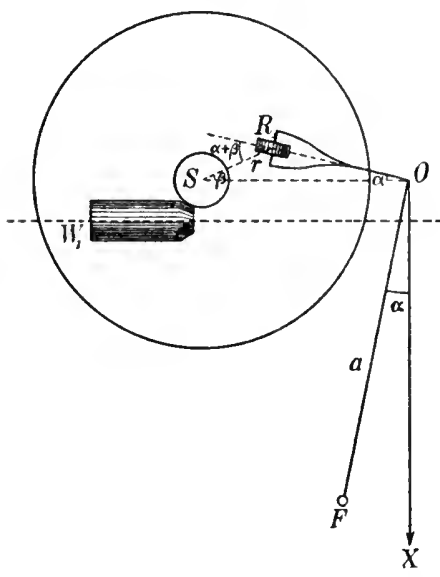
möge, um $R_{2} d \psi=\frac{R_{2}}{R_{1}} d x_{0}$. Ist der Radius dieses an der Scheibenachse sitzenden Zahnrädchens $R_{3}$, so ist seine Abwicklung $R_{3} d \varphi=R_{2} d \psi$, also der Winkel, um den

Fig. 50. sich die Scheibe dreht,

$$
d \varphi=\frac{R_{2}}{R_{1} R_{3}} d x_{0}=p d x_{0} .
$$

Wenn nun der Berührungspunkt der Rolle die Entfernung $r$ vom Scheibenmittelpunkt hat, so ist die $\mathrm{Be}$ wegung des Berührungspunktes $r d \varphi$, und wenn $\beta$ der Winkel von $r$ mit $S O$ ( $y$-Achse) und $\alpha$ der Winkel des Rollenarms mit der Richtung $O S$ ist, so beträgt die Abwicklung der Integrierrolle mit dem Radius $\varrho$ und dem Drehungswinkel $\omega$ :

$$
\varrho d \omega=r d \varphi \sin (\alpha+\beta)=r p \sin (\alpha+\beta) d x_{0} .
$$

Da nun $(O F=a$ gesetzt $)$

$$
x=x_{0}+a \cos \alpha, \quad y=a \sin \alpha
$$

die Koordinaten des Fahrstiftes sind, so ist wieder

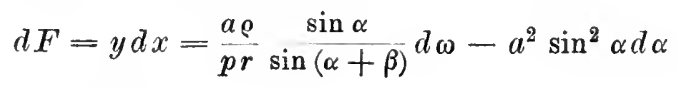

und mit Rücksicht auf

$$
r \sin (\alpha+\beta)=s \sin \alpha,
$$

wenn $s$ die konstante Entfernung des Drehpunktes $O$ vom Scheibenmittelpunkte $S$ ist:

Setzen wir noch

$$
F=\int \frac{a \varrho d \omega}{s p}-a^{2} \int \sin ^{2} \alpha d \alpha .
$$




$$
\frac{a R_{1} R_{3}}{s R_{\Xi}}=C, \quad \varrho d \omega=d \sigma,
$$

so wird für eine geschlossene Umfahrung:

$$
F=C\left(\sigma_{2}-\sigma_{1}\right) \text {. }
$$

Um eine Vorstellung von den Dimensionen zu erhalten, so gab nach Reitz ein Planimeter mit einem Fahrarm von $50 \mathrm{~mm}$ Länge für die Noniuseinheit den Flächenwert 0,2 qmm und man konnte, einen Streifen von $4 \mathrm{~cm}$ Breite ausmessen. Wurde der Fahrarm auf $125 \mathrm{~mm}$ verlängert, so betrug die Fläche, welche durch eine Einheit am Nonius gemessen wird, 0,5 qmm und die Breite des Streifens stieg auf $15 \mathrm{~cm}$.

Für die Theorie wurde angenommen, daB die Konstruktionsbedingungen erfüllt sind. Es kommt darauf an, die durch Abweichungen von diesen Bedingungen entstehenden Fehler zu untersuchen.

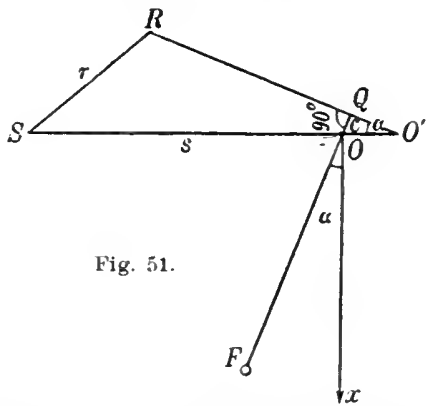

1. Wenn zwar die Achse der Integrierrolle dem Fahrarm parallel ist, aber die Rolle sich auf ihrer Achse verschoben hat, so geht die Ebene ihres Laufkreises nicht durch den Drehpunkt $O$ des Fahrarms hindurch, sondern an ihm vorbei und schneidet $F O$ in $Q$ (Fig. 51). Dann tritt an Stelle des Dreiecks $S O R$ das Dreieck $S O^{\prime} R$ mit den Winkeln $\alpha$ und $\beta^{\prime}$, wobei aber der Punkt $O^{\prime}$ je nach der Stellung des Fahrarms $F O$ und mit dem Winkel $\alpha$ sich verrückt und zwar so, daB $O Q=O O^{\prime} \sin \alpha=c$ konstant bleibt. Es war nun

oder wegen

$$
\rho d \omega=p r \sin (\alpha+\beta) d x_{0}
$$

wenn

$$
r \sin (\alpha+\beta)=s \sin \alpha,
$$

gesetzt wird,

$$
\frac{s R_{y}}{a R_{1} R_{3}}=s^{\prime}
$$

Da jetat

$$
\varrho d \omega=s^{\prime} y d x_{0} \text {. }
$$

$$
r \sin \left(\alpha+\beta^{\prime}\right)=\left(s+O O^{\prime}\right) \sin \alpha=s \sin \alpha+c
$$


wird, so ist jetzt

$$
\varrho d \omega=s^{\prime} y d x_{0}+c^{\prime} d x_{0} .
$$

Wenn dieser Ausdruck integriert wird, so fällt bei vollständiger Umfahrüng $c \int_{e} d x_{0}$ fort, und der in Rede stehende Fehler ist einfluBlos.

2. Ist die horizontale Projektion von $S O$ (der Verbindungsgeraden des Scheibenmittelpunktes und des Drehpunktes des Fahrarms) mit der Walzenachse $W_{1} W_{2}$ nicht genau parallel, sondern schließen beide den kleinen Winkel $\varepsilon$ miteinander ein, so entsteht ein Fehler.

Wir nehmen als $x$-Achse die Senkrechte auf

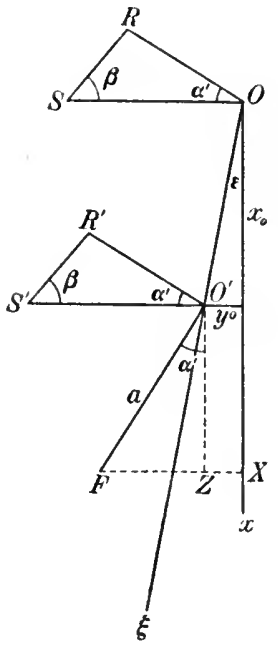

Fig. 52. der Anfangslage von $O S$ im Punkte $O$ und bezeichnen den Winkel des Fahrarms mit dieser Achse mit $\alpha^{\prime}$, während wir die zur Walzenachse senkrechte Fortbewegungsrichtung des Instrumentes mit $\xi$ bezeichnen. Durch Fortbewegung sei nun der Punkt $O$ längs der $\xi$-Achse in die Lage $O^{\prime}\left(x_{0}^{\prime} y_{0}^{\prime}\right)$ gekommen, $S^{\prime}$ sei dann der Scheibenmittelpunkt, $R^{\prime}$ der Berührungspunkt der Rolle mit der Scheibe. Im Dreiecke $R^{\prime} S^{\prime} O^{\prime}$ ist dann der Winkel bei $O^{\prime}$ gleich $\alpha^{\prime}$ und es besteht wie früher die Gleichung

$$
d \sigma=\rho d \omega=p r \sin \left(\alpha^{\prime}+\beta\right) d \xi_{0}
$$

oder wegen $r \sin \left(\alpha^{\prime}+\beta\right)=s \sin \alpha^{\prime}$

Nun ist

$$
d \sigma=p s \sin \alpha^{\prime} d \xi_{0} .
$$

und

$$
\cos \varepsilon=\frac{x_{0}}{\xi_{0}}, \quad \sin \varepsilon=\frac{y_{0}}{\xi_{0}}
$$

$$
\begin{aligned}
& x-x_{0}=a \cos \alpha^{\prime} \quad \text { oder } \quad x=a \cos \alpha^{\prime}+\xi_{0} \cos \varepsilon \\
& y-y_{0}=a \sin \alpha^{\prime} \quad \text { oder } \quad y=a \sin \alpha^{\prime}+\xi_{0} \sin \varepsilon
\end{aligned}
$$

Es wird also

$$
\begin{gathered}
d x=-a \sin \alpha^{\prime} d \alpha+\cos \varepsilon d \xi_{0} \\
d \xi_{0}=\frac{d \sigma}{p s \sin \alpha^{\prime}}=\sec \varepsilon\left(d x+a \sin \alpha^{\prime} d \alpha\right) \\
d \sigma=p s \cdot \sec \varepsilon\left(\sin \alpha^{\prime} d x+a \sin ^{2} \alpha^{\prime} d \alpha\right) \\
=\sec \varepsilon \cdot \frac{p s}{a}\left[\left(y-y_{0}\right) d x+a^{2} \sin ^{2} \alpha^{\prime} d \alpha\right]
\end{gathered}
$$


Bei der Integration über eine geschlossene Kurve fällt wie früber das letzte Glied fort, und es bleibt

$$
\sigma_{2}-\sigma_{1}=\sec \varepsilon \cdot \frac{p s}{a}\left[\int y d x-\int y_{0} d x\right] .
$$

AuBer dem Faktor sec $\varepsilon$ ist also noch ein Flächenintegral hinzugekommen. Diese fehlerzeigende Figur kann man sich von dem Punkte $Z$ beschrieben denken, welcher auf der Ordinate des Punktes $F$ durch die ron $O^{\prime}$ gefällte Senkrechte getroffen wird, indem $Z X$ $=y_{0}, O X=x$ die Koordinaten von $Z$ sind.

3. Ein weiterer Fehler entsteht, wenn die Achse der Integrierrolle dem Fahrarm nicht parallel ist oder $O^{\prime} R^{\prime}$ nicht zu $O^{\prime} F$ senkrecht ist.

Es sei $R^{\prime} O^{\prime} F^{\prime}=90^{\circ}+\varepsilon$, wo $\varepsilon$ klein sein soll, so legen wir in der Anfangsstellung des Instrumentes die $x$-Achse so, da $B$ sie mit $S O$ den Winkel $90^{\circ}+\varepsilon$ einschlieBt und mit der Fortbewegungsrichtung des Instrumentes ( $\xi$-Achse) den Winkel $\varepsilon$ bildet. Für eine beliebige Stellung des Instrumentes ist dann der Winkel $R^{\prime} O^{\prime} S^{\prime}=\alpha^{\prime}$ dem Winkel des Fahrarms mit der $x$-Achse gleich. Wir orhalten also eine ganz ähnliche Figur wie im vorigen Falle (nur daB $R^{\prime} O^{\prime} F$ kein rechter Winkel ist).

Wenn $F$ auf der andern Seite der $x$-Achse liegt, so liegen $F$ und $Z$ auf rerschiedenen Seiten derselben, d. b. das Vorzeichen der hinzutretenden Fläche ändert sich. Man kann also durch Umfahrung einer Fläche von bekanntem Inhalt (einmal auf der einen, das andre Mal auf der andern Seite der $x$-Achse) die GröBe der feblerzeigenden Figur als halbe Differenz der Ergebnisse ermitteln. Als Gebrauchsrorschrift für das Instrument ergibt sich zugleich die Regel, daB für die auszumessende Figur die Bewegungsrichtung des Mittelpunktes des Instrumentes nahezu eine Symmetrielinie sein soll.

4. Wenn die Walzen nicht genau gleiche Durchmesser haben oder die Drehachse nicht durch den Scbwerpunkt des Instrumentes geht und die Riffelung der Walzen keinen hinreichenden Widerstand gegen den exzentrisch ausgeübten Zug des Fahrarms bietet, so wird der Drehpunkt keine gerade Linie senkrecht zur Walzenachse in ihrer Anfangslage, sondern einen Kreisbogen beschreiben.

Zur Anfangslage $W_{1} W_{2}$ sei die $x$-Achse durch $O$ senkrecht gelegt. In der Lage $W_{1}^{\prime} W_{2}^{\prime \prime}$ schlieBe die Senkrechte zur Walzenachse den Winkel $\gamma$ mit der $x$-Achse ein; den Übergang zu einer 
benachbarten Lage $W_{1}^{\prime \prime} W_{2}^{\prime \prime}$ kann man in eine Parallelbewegung und eine Drehbewegung um $\mathrm{O}_{2}$ zerlegt denken. Bei der Parallelbewegung, die senkrecht zur Walzenachse erfolgt, wird der Bogen $d s=\sec y d x_{0}$ abgewickelt und von der Integrierrolle

Da ferner

$$
d \sigma=p a \sin \alpha d s=p a \sin \alpha \sec \gamma d x_{0} .
$$

und

$$
x=x_{0}+a \cos (\alpha+\gamma)
$$

so wird

$$
d x=d x_{0}-a \sin (\alpha+\gamma) d(\alpha+\gamma),
$$

$d \sigma=p a \sin \alpha \sec \gamma d x+p a^{2} \sin \alpha \sec \gamma \sin (\alpha+\gamma) d(\alpha+\gamma)$.

Wenn man annehmen darf, da $\beta$ das Instrument bei vollständiger Umfahrung einer Figur in seine anfängliche Lage zurückkehrt, so verschwindet bei der Integration der zweite Teil dieses Ausdrucks. Im ersten Teil ist $a \sin \alpha \sec \gamma$ derjenige Teil der Ordinate des Fahrpunktes, der zwischen diesem und der Bahntangente des Punktes $O_{2}$ liegt. Ist $S$ der Schnittpunkt der Bahntangente mit der Ordinate, so ist der geometrische Ort des Punktes $S$ die Umgrenzung eines Flächenstückes, das um so kleiner ist, je kleiner der Winkel $\gamma$ ist. Man kann daher diese Figur wiederum als fehlerzeigende betrachten, die von der Integrierrolle algebraisch zu der auszumessenden Fläche addiert wird. Auch hier ändert sich das Vorzeichen, wenn $F$ auf verschiedenen Seiten der $x$-Achse liegt und der Fehler wird am wenigsten EinfluB haben, wenn die Senkrechte zur Walzenachse eine Symmetrielinie der auszumessenden Fläche ist.

A msler hat darauf hingewiesen, da $B$ bei einer auf einer Ebene aufliegenden Rolle ein Gleiten nicht gänzlich beseitigt werden kann und dadurch die Messung verfälscht wird. Man ersetzt deshalb die Rolle dưrch einen Zylinder und die Ebene durch eine. Kugelfläche (S. 52).

Das mit dieser Vorrichtung versehene Planimeter wird als $\mathrm{Ku}$ gelrollplanimeter bezeichnet (Fig. 53). Es ist ganz ähnlich dem vorher beschriebenen konstruiert. In die eine Walze $R$ sind am Umfange Zähne eingeschnitten, welche in ein darüber befindliches vertikales Zahnrad eingreifen, dessen Drehungsachse mit der Walzenachse $A$ parallel ist und senkrecht über ihr liegt. Diese Achse trägt an ihrem in der Mitte des Instruments befindlichen Ende ein Kugelsegment $K$, dessen Mittelpunkt in ihr liegt. An das glockenartige 
Kugelsegment, dessen ebene Endfläche senkreeht zur horizontalen Walzenachse steht, legt sich durch Federdruck ein horizontaler um seine Achse drehbarer Zylinder an, dessen Umdrehungen nebst ihren Bruchteilen an einem besonderen Zählwerk abgelesen werden. Mit dem Zylinderlager (dem Rahmen $M$, der den Zslinder trägt) ist der Fahrarm verbunden, der der Zylinderachse parallel liegt. Der Zylinder soll die Kugelfläche in ihrem gröBten Horizontalkreise berühren. Befindet sich der Fahrarm in seiner Grundstellung, nämlich senkrecht zur Walzenachse und damit auch senkrecht zur Achse des Kugelsegments, so findet die Berührung ron Kugel und

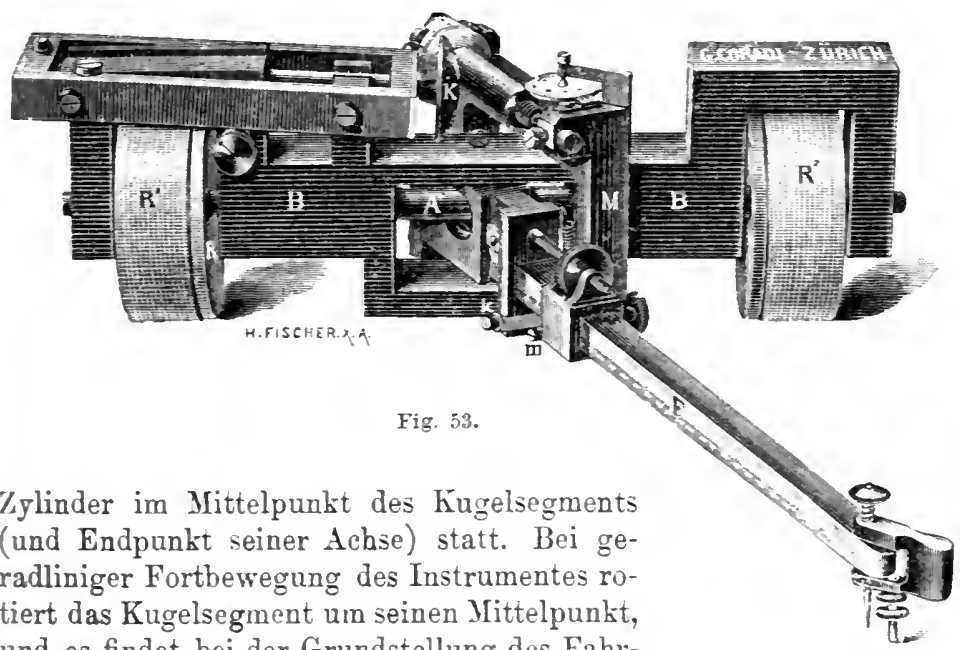
und es findet bei der Grundstellung des Fahrarms keine Bewegung des Zylinders statt. Aber es entsteht auch, wenn die Berührung bei der Grundstellung des Fahrarms etwas oberhalb oder unterhalb der Vitte des Kugelsegments stattfindet, keine Rotation des Zylinders durch Bewegung des Wagens, sondern nur eine geringe Bewegung parallel der Zylinderachse. Wenn dagegen der Fahrarm um seine vertikale Drehachse bewegt wird, so wird eine geringe Umdrehung des Zylinders verursacht, falls die Berührung nicht auf dem größten Horizontalkreise des Segments bleibt. ${ }^{2}$ ) Die MeBrolle tritt dagegen in Tätigkeit, sobald das ln-

1) Diese Drehung wird eine Funktion des Winkels $\alpha$ sein, den der Fahrarm mit seiner Grundstellang bildet. Bei der Umfahrung 
strument bewegt wird und der Fahrarm außerhalb der Normalstellung steht.

Bezeichnen wir den Radius der Walzen $R^{1}, R^{2}$ mit $W$, so ist ihre Abwicklung $W d \psi=d x_{0}$ gleich dem von dem Drehpunkt des Fahrarms zurückgelegten Wegelement. Das in die Walze eingreifende Zahnrad mit dem Radius $r^{\prime}$ wird eine Drehung um $d \varphi$ erleiden, die durch $r^{\prime} d \varphi=W d \psi=d x_{0}$. bestimmt ist. Da der Drehungswinkel des Kugelsegmentes ebenfalls $d \varphi$ ist, so können wir die auf S. 51 gegebene Entwicklung anwenden. Wir nehmen an, der Berührungspunkt $P$ des Zylinders stehe vom Mittelpunkt $C$ des Segmentes oder Endpunkte der Achse um $r \delta$ im Bogen größten Kreises ab. Der Bogen des durch $P$ gehenden Vertikalkreises, der durch den obersten Puukt $Z$ der vervollständigten Kugel geht, ist dann, da seine Ebene die Zylinderachse senkrecht durchschneidet, als Rollenebene (im Sinne obiger Entwicklung) zu betrachten. Der Winkel, den $P Z$ und $P C$ einschließen, sei $\gamma$. Dann ist die Abwicklung des Zylinders $\varrho d \omega=r \sin \delta \sin \gamma d \varphi$. Nun ist der Winkel der Vertikalebene $Z \dot{P}$ mit der Vertikalebene $Z C$ der Kugelachse gleich dem Winkel $\alpha$ des Fahrarms mit seiner Grundstellung. Es ist also $\sin \alpha=\sin \delta \sin \gamma$, mithin

Führen wir wieder

$$
\varrho d \omega=r \sin \alpha d \varphi=\frac{r}{r^{\prime}} \sin \alpha d x_{0} .
$$

$$
\begin{aligned}
& \quad a \sin \alpha=y, \quad a \cos \alpha=x-x_{0}, \quad d x_{0}=d x-a \sin \alpha d \alpha \\
& \text { ein, so folgt } \quad \rho d \omega=\frac{r}{r^{\prime} a} y d x-\frac{r}{r^{\prime}} a \sin ^{2} \alpha d \alpha
\end{aligned}
$$

und bei der Integration über eine geschlossene Kurve

$$
F=\frac{a r^{\prime}}{r}\left(\sigma_{2}-\sigma_{1}\right)
$$

Die Prüfung des Instrumentes wird am einfachsten in der Weise ausgeführt, daB man einmal dieselbe Fläche in verschiedenen Lagen zur Abszissenachse und zweitens Figuren mit verschiedener Umgrenzung, aber mit gleichem Flächeninhalt umfährt. Wenn

einer geschlossenen Figur wird das Integral $\int f(\alpha) d \alpha$ nahezu verschwinden, wenn eine Rückkehr zu demselben Ausgangwert von $\alpha$ stattfindet, da $f(\boldsymbol{c})$ dieselben Werte mit anderen Zeichen durchlaufen wird. 
diese Unabhängigkeit von Lage und Gestalt innerhalb der Grenzen der Genauigkeit nicht vorhanden ist, so lassen sich im allgemeinen die Konstruktionsmängel nur durch den Mechaniker beseitigen. Es ist nur eine Berichtigungsvorrichtung vorhanden, welche den Rahmen der Integrierrolle im horizontalen Sinne zu verstellen gestattet, um den Parallelismus von Zylinderachse und Fahrarm herzustellen ${ }^{1}$ ), während die Horizontalität der Zylinderachse, die eine besonders wichtige Bedingung ist, als mechanisch erreichbar stillschweigend vorausgesetzt wird.

Die Konstante bestimmt man bei diesen Instrumenten am sichersten durch Umfabren einer Figur von bekanntem Flächeninhalt z. B. mit Benutzung eines Kontrollineals (s. o. S. 84). Sowohl bei der Justierung (durch Einstellung des Fahrarms auf eine gewünschte Länge durch Schraube $m$, die der Konstanten einen runden Wert erteilt), wie bei der Anwendung des Instrumentes zur Flächenmessung ist es empfehlenswert, die Umfahrung in solcher Stellung zu beginnen, wo die Mebrolle eine geringe oder gar keine Bewegung erleidet, also bei der Grundstellung des Fahrarms, weil hierbei eine kleine Unsicherheit in der Anfangs- und Endlage keinen bemerkenswerten Einfluß anf die Ablesung ausübt und ebenso wenig eine anfängliche Trägheit der Rollenbewegung bemerkbar wird.

40. Polarplanimeter. Mit der Ausbildung der Koordinatenund Linearplanimeter ist die Entwicklung der Polarplanimeter parallel vor sich gegangen.

Das Polarplanimeter lehnt sich in seiner Konstruktion an das Vorbild des menschlichen Armes (bei Horizontalbewegung desselben) an, indem wie der Oberarm im Schultergelenk, der Polarm sich um einen festen Punkt als Pol dreht. Mit ihm ist durch ein Scharnier, das dem Ellbogengelenk entspricht, der dem Unterarm vergleichbare Fahrarm verbunden, der die Hülse für den Fahrstift wie die Hand die Feder trägt. Die Bewegungen des Fahrarmes senkrecht zu seiner Richtung werden wiederum durch die Abwicklung

1) Wenn die Zylinderachse mit dem Fahrarm in der Horizontalprojektion einen Winkel $\varepsilon$ bildet, so erhält man ähnlich wie beim vorigen Instrument, abgesehen von einem konstanten Faktor $\cos \varepsilon$, den richtigen Flächeninhalt als Mittel aus 2 Umfahrungen zu beiden Seiten der $x$-Achse, und es folgt wieder als Regel, die aus\%umessenden Figuren symmetrisch zur $x$-Achse zu legen (langgestreckte Rechtecke und ähnlicbe Figuren in schrïger Lage), um das auftretende Integral $\int \cos \alpha d x$ möglichst geringen EinfluB gewinnen zu lassen. 
einer MeBrolle gemessen. Das eine Ende des Fahrarms wird in einem Kreishogen mit dem Polarm als Radius geführt, während das andere Ende die auszumessende Figur umfährt.

Die älteste Idee eines Polarplanimeters stammt aus dem Jahre 1814 von dem Trigonometer Hermann in München, es wurde dann unabhängig von A. Miller von $\mathrm{Hauenfels}$ in Leoben 1855 und wahrscheinlich ein Jahr früher von Jakob Amsler erfunden, der zwar seine Erfindung erst 1856 veröffentlichte, tatsächlich aber derjenige ist, der das Polarplanimeter in die Praxis einführte und damit alle andern Planimeter zurückdrängte.

Endlich hat auch Clark Maxwell fast gleichzeitig ein Polarplanimeter konstruiert, das dadurch bemerkenswert ist, daß eine Kugel auf einer anderen sich abwälzt, eine Einrichtung, die wohl bei keinem andern Planimeter verwendet worden ist. Auch ist von Buniakowsky in Petersburg und Decker in Augsburg ein Polarplanimeter erfunden worden, das aber ebensowenig wie das vorhergehende in Konkurrenz mit A mslers Konstruktion getreten ist.

In der allgemeinen Formel $F=A-B$ (S. 73) ist beim Polarplanimeter $A$ die vom Fahrstift umschriebene Fläche, $B$ ist durch die vom Gelenk zwischen Fahrarm und Polarm beschriebene Kreiskurve begrenzt und $F$ ist der vom Fahrarm überstrichene und durch die Rollenablesung gegebene Flächeninhalt. Je nachdem der Gelenkpunkt, den wir im folgenden mit $O$ bezeichnen wollen, einen Kreisbogen hin und zurück oder einen vollen Kreis beschreibt,

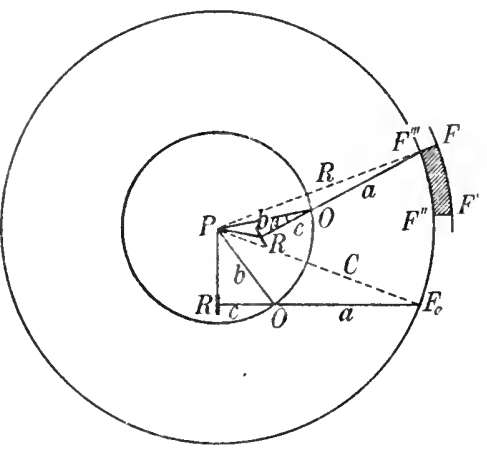

Fig. 54. wird $B$ Null oder der Fläche eines Kreises gleichzusetzen sein. Um aber Fehlerbetrachtungen anschließen zu können und auf die besonderen Konstruktionen der Instrumente Rücksicht zu nehmen, ist es wie bei den früheren Planimetern nötig, die Theorie genauer zu entwickeln.

Es sei (Fig 54) $P$ der Pol, $P O=b$ der Polarm, $O F=a$ der Fahrarm, $P F=R$ der Leitstrahl des Fahrstiftes. Die MeBrolle $R$ ist gewöhnlich in der Rückwärtsverlängerung des Fahrarms angebracht, und es sei $\dot{O} R=c$. Ist sie zwischen $O$ und $F$ gelegen, so $m u B c$ mit negativem Zeichen ein- 
geführt werden. Im ersten Falle kann der Fahrstift $F$ zu beiden Seiten des Fahrarms nur Bögen von nahezu $180^{\circ}$ umfahren.

Lassen wir $F$ einen Kreisbogen $F F^{\prime}$ um den Pol als Mittelpunkt beschreiben, so bleibt für konstantes $R$ das Dreieck $P O F$ unverändert und das ganze Instrument wird ohne Veränderung der Lage des Fahrarms zum Polarm um den Zentriwinkel $\varphi$ des Kreisbogens herumgeschwenkt. Die Rolle $R$ beschreibt infolgedessen ebenfalls einen Kreisbogen um $P$ mit demselben Zentriwinkel $\varphi$, dessen Radius $r$ sei. Wenn die Rollenachse, die dem Fahrarm parallel liegt, mit dem Radius $P R$ den während dieser Bewegung unveränderlichen Winkel a einschlieBt, so beträgt ihre Abwicklung, wenn wie früher $\varrho$ der Radius, $\omega$ der Drehungswinkel der Rolle um ihre Achse ist, $\sigma=\rho \omega=r \varphi \cos \alpha$. Wird noch der Winkel zwischen Polarm und (verlängertem) Fahrarm mit $P O R=\beta$ bezeichnet, so ist $c=b \cos \beta-r \cos c$, mithin

$$
\begin{gathered}
\sigma=(b \cos \beta-c) \varphi \text { und da } R^{2}=a^{2}+b^{2}+2 a b \cos \beta \text { ist, } \\
\sigma=\left(R^{2}-a^{2}-b^{2}-2 a c\right) \frac{\varphi}{2 a} .
\end{gathered}
$$

Führt man noch die Bezeichnung $C^{2}=a^{2}+b^{2}+2 a c$ ein, so wird

$$
a \sigma=\frac{1}{2}\left(R^{2}-C^{2}\right) \varphi \text {. }
$$

$C$ kann man als den besonderen Wert ron $R$ auffassen, für den $c=b \cos \beta$, also $r \cos \alpha=0, \alpha=90^{\circ}$ und $\sigma=0$ ist. Dies findet dann statt, wenn $P R$ zu $F_{0} R$ senkrecht steht (unten in Fig. 54) oder mit anderen Worten, wenn der Fahrarm so gestellt ist, daB die Ebene der Rolle durch den Pol geht. Man nennt den Kreis mit dem Radius $C$ den Grundkreis. Bei der Bewegung des Fahrstiftes auf dem Grundkreis $(R=C)$ findet keine Rollenabricklung statt. Führt man den Fahrstift dagegen auf dem Kreisbogen $F F^{\prime}$, so folgt aus obiger Gleichung, daB die mit der Fahrarmlänge multiplizierte Rollenabwicklung der Differenz der Kreissektoren mit dem Zentriwinkel $\varphi$ und den Radien $R$ und $C$ gleich ist, $d . \mathrm{b}$. dem Kreisringstück $F F^{\prime} F^{\prime \prime} F^{\prime \prime \prime}$ zwischen dem beliebig gewählteu Kreise und dem Grundkreise, das durch die Radienvektoren am Anfang und Ende der Bewegung begrenzt ist. Dieselbe Rollenabwicklung würde man aber auch bei Umfahrung des Kreisring. stückes $F F^{\prime} F^{\prime \prime} F^{\prime \prime \prime}$ erhalten, da bei Führung des Stiftes auf den radialen Strecken $F^{\prime} F^{\prime \prime}$ und $F^{\prime \prime \prime} F$ die Rolle dieselben Cimdrehungen aber in entgegengesetztem Sinne macht, also die Abwicklung sich 
aufhebt und längs $F^{\prime \prime} F^{\prime \prime \prime}$ auf dem Grundkreise die Rolle unbewegt bleibt.

Eine beliebige Fläche kann man sich von einer Kurve begrenzt denken, die aus unendlich kleinen Kreisbogenstücken und radialen Strecken in bezug auf $P$ als Mittelpunkt besteht. Umfährt der Punkt $F$ diese Begrenzungskurve, so bestreicht der Radiusvektor $P F$ Kreissektoren vom Flächeninhalte $\frac{1}{2} R^{2} d \varphi$. Bildet man das Integral $\frac{1}{2} \int R^{2} d \varphi$, so stellt dieses den Inhalt der betrachteten Fläche dar, indem man bei Berücksichtigung des Vorzeichens von $d \varphi$ übersieht, daB die außerhalb der begrenzten Fläche gelegenen Teile zweimal mit verschiedenem Vorzeichen auftreten. Es ist daher für eine beliebige. Fläche

oder wenn wir

$$
\text { a } \int d \sigma=\frac{1}{2} \int R^{2} d \varphi-C^{2} \int d \varphi,
$$

$$
\frac{1}{2} \int R^{2} d \varphi=F
$$

setzen,

$$
F=a\left(\sigma_{2}-\sigma_{1}\right)+C^{2}\left(\varphi_{2}-\varphi_{1}\right) .
$$

$\varphi_{2}$ und $\varphi_{1}$ sind die den Stellungen des Polarms am Anfang und Ende der Umfahrung entsprechenden Winkel mit einer beliebig gewählten Anfangsrichtung. Kehrt der Polarm bei vollständiger Umfahrung von $F$ in seine Anfangslage zurück, so ist wegen $\varphi_{2}=\varphi_{1}$ das letzte Glied Null. Liegt der Pol aber innerhalb der auszumessenden Figur, so wird der Polarm nach Umfahrung derselben einen vollen Umlauf um den $\mathrm{Pol}$ gemacht haben und

$$
\varphi_{2}-\varphi_{1}=2 \pi
$$

werden. In diesem Falle ist

$$
F=a\left(\sigma_{2}-\sigma_{1}\right)+C^{2} \pi \text {. }
$$

$C^{2} \pi$ ist aber die Fläche des Grundkreises, die demnach zu der mit der Fahrarmlänge multiplizierten Ablesungsdifferenz der Rolle addiert werden muB, wenn der Pol im Innern liegt.

Ein Mangel des gewöhnlichen Polarplanimeters ist die Bewegung der Rolle auf der Planunterlage, so daß infolge kleiner Unebenheiten ein unregelmäBiges Abwälzen erfolgt. ${ }^{1}$ ) Dieser Mangel

1) Dieser Mangel ließe sich einfach dadurch beseitigen, da $B$ man die MeBrolle auf einem nicht zu breiten Kreisringstück (von etwa 
ist beim Präzisionsplanimeter behoben, indem die Rolle auf einer Scheibe läuft. Die älteste Konstruktion von Hohmann und Coradi hatte eine senkrecht stehende Scheibe, gegen welche die Mebrolle gedrückt wurde. Da hierbei keine genügende Sicherheit des Rollens gewährleistet war, so wurde bei einer zweiten Konstruktion die Scheibe schräg gestellt.

In der Verlängerung des Polarms (Fig. 55) ist die schiefliegende Achse einer Laufrolle $L$ angebracht, die daher bei der Drehung des Instrumentes einen Kreisbogen um den Pol $P$ beschreibt. Auf $\underline{P}$ derselbèn Achse sitzt die kreisförmige Scheibe $S$, welche ebenfalls schief und parallel zur Laufrolle steht. Senkrecht zum Fahrarm $a$ ist in dem Drehpunkte O, der Fahrarm und Polarm verbindet, ein kurzer Arm $c$ angebracht, welcher einen die Mebrolle tragenden Schlitten in der zum Polarm

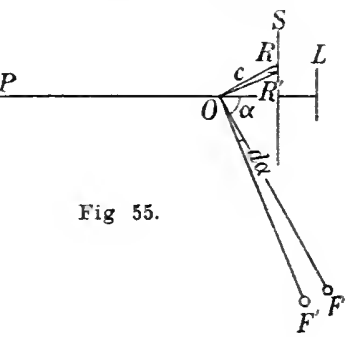
$P I=l$ senkrechten Richtung verschiebt, so daB die Verschiebung der Mebrolle in dem zum Schlitten parallelen (horizontalen) Durchmesser der Scheibe stattfindet, wenn der Fahrarm sich um $O$ dreht.

Ist $\propto$ der Winkel des Fahrarms mit der Verlängerung $O L$ des Polarms $P O$, so ist $\delta=c \cos \alpha$ der Abstand der Rolle rom Mittelpunkt der Scheibe und bei einer Drehung des Fahrarms um il verschiebt sich der Berührungspunkt der Rolle um $d \delta=-c \sin \omega d \alpha$. Beschreibt der Fahrstift einen Kreisbogen mit dem Radius $r$ um $P$, so ist $r^{2}=a^{2}+b^{2}+2 a b \cos \varsigma$. Der Winkel, um den sich sowohl $r$ als der Polarm dreht, sei $d \psi$, dann wickelt die schief stehende Laufrolle mit dem Radius $L$, da ihr Berührungspunkt mit der Unterlage den Bogen $l d \psi$ beschreibt, den Bogen $L d \lambda=$ $l d \psi$ ab. $d \lambda=\frac{l}{L} d \psi$ ist der Drehungswinkel der Laufrolle und gleichzeitig auch der Scheibe. Daher ist $\delta d \lambda=\delta_{\frac{L}{L}}^{l} d \psi$ die Bogenstrecke, um die der Berührungspunkt der Integrierrolle auf der Scheibe weiterrückt und die Abwicklung der Integrierrolle ist

$120^{\circ} \mathrm{WinkelgröBe)}$ laufen lieBe, das etwa nur durch eine bei $60^{\circ} \mathrm{ge}$ legte radiale Schiene mit dem Pole verbunden wäre. Das Instrument kőnnte so gebaut sein, dab das Gelenk zwischen Pol- und Fahrarm möglichst über dem Stützpunkt der Rolle läge, wodurch die überflüssigen Bewegungen der Rolle auf ein Minimum beschränkt würden. 


$$
d \sigma=\varrho d \omega=\delta d \lambda
$$

oder mit Einführung von $p=c \frac{l}{L}$

$$
d \sigma=p \cos \alpha d \psi \text {. }
$$

Während bei diesem Planimeter die schrägstehende Rolle die Drehung des Polarmes um den Pol auf die Scheibe überträgt, wird bei einer zweiten Konstruktion von Hohmann-Coradi die Drehung der Scheibe dadurch hervorgebracht, daB ein unter ihrem Mittelpunkt sitzendes Triebrad an dem geriffelten Rande einer den Pol umgebenden kreisrunden Polplatte entlang läuft. Die Ausführung ist in zwei verschiedenen Arten erfolgt.

Bei dem freischwebenden Präzisionsplanimeter ist im Mittelpunkte der geriffelten Polplatte $P$ eine vertikale Achse eingesteckt. Der an einem Ende gabelförmige Polarm umfaßt mit der Gabel die Polachse und wird durch eine von der oberen Spitze der Polachse nach seinem anderen Ende gehenden Lamelle getragen. Am Polarm ist die vertikale Achse einer an der Riffelung der Polplatte entlang laufenden Laufrolle $L$ angebracht. Über $L$ sitzt auf derselben Achse die mit Papier überzogene Hartgummischeibe $S$ (vgl. Fig. 56). Am anderen Ende des Polarms ist um eine Vertikalachse drehbar die Hülse angebracht, in der sich der Fahrarm verschieben läBt, der mit einer Teilung (in halbe Millimeter) versehen, mit Hilfo eines Nonius auf eine gewünschte Länge eingestellt und mit einer Klemmschraube festgeklemmt werden kann.

Mit der Hülse steht ein Rahmen in fester Verbindung, der die auf der Scheibe aufliegende MeBrolle trägt, deren Achse dem Fahrarm parallel ist. Wenn der Fahrarm und Polarm senkrecht zu einander stehen, soll die Berührung im Scheibenmittelpunkt stattfinden. Die Bewegung des Fahrarms allein hat, da die Rollenebene senkrecht zu ihm steht, eine nur gleitende Bewegung der MeBrolle zur Folge; denn der Arm, welcher die MeBrolle trägt, dreht sich um den Drehpunkt der Hülse, und der Berührungspunkt beschreibt einen Kreis um diesen Drehpunkt. Wird dagegen der Polarm bewegt, so setzt sich die Scheibe in drehende Bewegung und diese überträgt sich auf die MeBrolle, die z. T. gleitende, z. T. rollende Bewegungen ausführt, deren GröBe von ihrer Lage zum Mittelpunkt der Scheibe abhängt.

Sei wieder $d \psi$ die Drehung des Polarms, $P$ der Halbmesser der Polscheibe, $L$ derjenige der Laufrolle, $d \lambda$ ihr Drehungswinkel, 
so ist $P d \psi=L d \lambda . d \lambda$ ist zugleich der Drehungswinkel der Scheibe, und wenn die MeBrolle den Abstand $\delta$ rom Scheibenmittelpunkt hat und $\alpha$ der Winkel ist, den der Fahrarm mit der Verlängerung des Polarms, ferner $\beta$ der Winkel, den der Radius $\delta$ mit der Richtung des Polarms einschliebt, so ist die Abwicklung der JeBrolle $d \sigma=\delta d \lambda \cdot \cos (\alpha-\beta)$. Ist $c$ die Entfernung des Scheibenmittelpunktes rom Drehpunkt des Fahrarms, so ist $\delta \cos (\alpha-\beta)=c \cos \alpha$ oder wenn wir $c \frac{P}{L}=p$ setzen,

$$
d \sigma=p d \psi \cos c \text {. }
$$

Es möge hier gleich bemerkt werden, daß dieses Instrument für die Lage des Pols im Innern einer Figur nicht eingerichtet ist, und daB nur ein Teil des Cmfanges der Polplatte geriffelt ist.

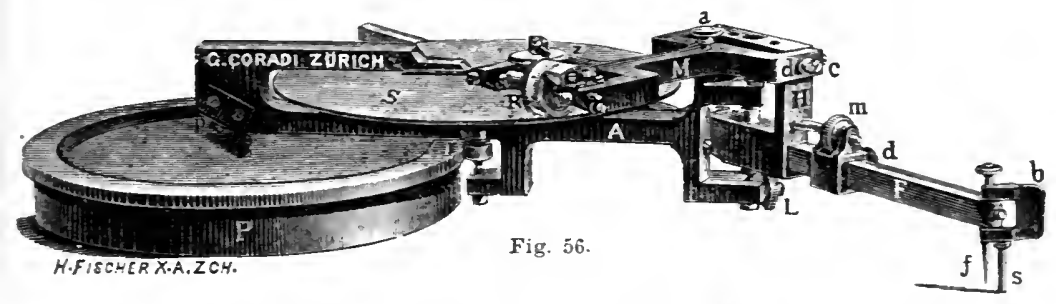

Bei der anderen Konstruktion (Fig. 56) ist die Aufhängevorrichtung weggelassen und durch eine Tragrolle $L$ ersetzt, die so angebracht ist, daB das Übergewicht des Instrumentes auf der Seite des Fahrstifts liegt. Dieses als einfaches Präzisionsplanimeter bezeichnete Instrument ist nur in kleinen Dimensionen ausgeführt und eignet sich also nur zur Ausmessung ron kleineren Flächen.

Bei der dritten Klasse von Planimetern ron Hohmann-Coradi, den Kugelpolarplanimetern, ist die Scheibe durch eine Kugelfläche mit horizontaler Drehachse ersetzt. An die Stelle der MeB. rolle tritt eine zylindrische Integrierwalze, welche gegen die Kugelfläche. gedrückt wird.

Beim einfachen Kugelpolarplanimeter läuft die am Ende des Polararms angebrachte Kugelschale auf der Zeichenunterlage, beim freischwebenden Kugelpolarplanimeter ist unterhalb des Polarms, durch ein Gegengewicht ausbalanziert, eine ihm parallele Achse mit der Kugelkalotte am Ende angebracht. Diese Achse wird durch ein auf dem fein gezahnten Rande der Polplatte laufendes Rädchen in Umdrehung versetzt. Am an- 
deren Ende des Polarmbügels befindet sich die um eine vertikale Achse drehbare Hülse, die den verschiebbaren Fahrarm aufnimmt; der Fahrarm trägt den Rahmen für die durch eine Feder an die Kugel gedrückte Zylinderwalze, deren Achse dem Fahrarm parallel ist (vgl. Fig. 53).

Bei diesem Planimeter ist wiederum eine völlige Unabhängigkeit der Meßvorrichtung von den Unebenbeiten der Unterlage gewäbrleistet. Durch die Verwendung der Kugelfläche ist außerdem ein Gleiten der Integrierwalze ausgeschlossen. Wird bei festgehaltenem Polarm der Fahrarm gedreht, so erfolgen nur ganz geringe Drehungen des Zylinders, insofern dieser die Kugel nicht ganz genau im größten Horizontalkreis berührt und nicht genau horizontal liegt. Diese sind aber bei geschlossenen Figuren einfluBlos (siehe S. 97). Die Drehung des Polarms hat dagegen eine Drehung der Kugelfläche zur Folge, welche nur dann keine Drehung der Integrierwalze verursacht, wenn diese die Kugel im Endpunkt ihrer Umdrehungsachse berührt, d. h. wenn der Fahrarm zum Polarm senkrecht steht, das Instrument sich also in seiner Grundstellung befindet. Je weiter der Berührungspunkt von diesem Kugelpol abliegt, um so größere Drehungen erleidet die Walze.

Bildet der Fahrarm mit dem verlängerten Polarm den Winkel $\alpha$, so berührt der Zylinder die Kugel in einem Punkte des Horizontalkreises der Kugel, dessen Radius mit dem Polarm (oder mit der Umdrehungsachse der Kugel) den Winkel $90^{\circ}-\alpha$ einschließt, da die Zylinderachse dem Fahrarm parallel ist. Bei der Drehung der Kugel beschreibt der Berührungspunkt auf ihr einen kleinen, zur Umdrehungsachse senkrechten Kreis, dessen Radius daher $R \cos \alpha$ ist, wenn mit $R$ der Kugelradius bezeichnet wird.

Beschreibt der Fahrstift $F$ einen kleinen Kreisbogen, so beschreibt der Leitstrahl und ebenso der Polarm (dessen Steliung zum Fahrarm in diesem Falle unverändert bleibt) einen Winkel $d \psi$. Die Rolle mit dem Radius $L$ rollt an dem Bogenstück $P d \psi$ der kreisförmigen Polplatte mit dem Radius $P$ entlang und dreht sich um einen Winkel $d \lambda$, der durch $P d \psi=L d \lambda$ bestimmt ist. Um denselben Winkel $d \lambda$ dreht sich aber die auf derselben Achse sitzende Kugelschale, also legt ein Punkt des Berührungskreises auf der Kugel den Weg $R \cos \alpha d \lambda$ zurück, dem die Abwicklung des Zylinders $d \sigma=\rho d \omega$ gleich ist ( $\varrho$ Zylinderradius, $d \omega$ sein Drehungswinkel). Man erhält also, wenn noch $R \frac{P}{L}=p$ gesetzt 
wird und der Fahrstift einen Kreisbogen um den Pol beschreibt,

$$
d \sigma=p \cos \alpha d \psi \text {. }
$$

Wir sehen also, daB bei allen diesen Planimetern für die an einem besonderen Zählapparat abgelesenen Umdrehungen der Rolle oder des Zylinders dieselbe Formel gilt, und da B nur der konstante, von den Dimensionen abhängige Faktor $p$ je nach der besonderen Anordnung verschieden ist.

Wenn nun der Fahrstift einen beliebigen kleinen Weg $F F_{\mathbf{z}}$ zurücklegt, bei dem der Leitstrahl $P F=r$ einen kleinen Winkel $d \varphi$ überstreicht, so denken wir uns den Weg in zwei Teile $F F_{1}$ und $F_{1} F_{2}$ zerlegt (Fig. 57.). Der erste Teil wird durch die Bewegung des Polarms allein durchlaufen, indem $F F_{1}$ ein Teil eines Kreisbogens ist, wobei die gegenseitige Lage von Polarm und Fahrarm unverändert bleibt. Das ganze Instrument, also auch der Polarm wird um den Winkel $d \psi$ herumgeschwenkt. Der zweite Teil der Bewe-

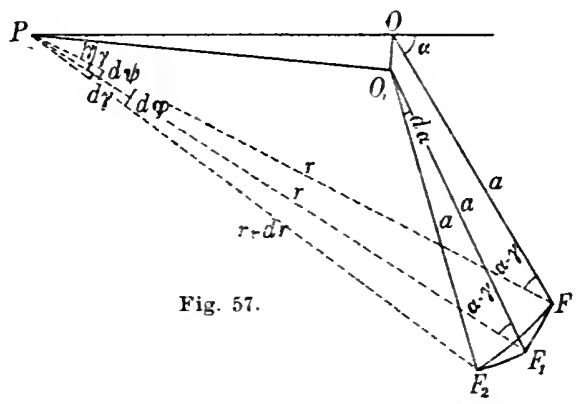
gung, $F_{1} F_{2}$, wird durch Drehung des Fahrarms für sich hervorgebracht und der Leitstrahl $P F$ überstreicht einen Winkel $d \gamma$. Der ganze bei der Bewegung des Fahrstifts ron $F$ bis $F_{q}$ vom Leitstrahl überstrichene Winkel ist dann $d \varphi=d \psi+d \gamma$.

Den Winkel $d \psi$ erhalten wir, wie wir gesehen haben, durch die Rollenabwicklung $d \sigma$. Es kommt nuu noch darauf an $d \gamma$ durch $d \alpha$, den Winkel, um den sich der Fahrarm dreht, auszudrücken. Wendet man auf das Dreieck POF, dessen drei Winkel $\gamma, 180^{\circ}-\alpha$ und $a-\gamma$ sind (da wir $\gamma$ von der Anfangslage des Polarms aus zählen dürfen), eine bekannte Differentialformel an, so erhält man

$$
r d \gamma=\sin (\alpha-\gamma) d a-\sin \gamma d b-a \cos (\alpha-\gamma) d \alpha
$$

oder da $a$ und $b$ konstante Längen sind, und $-\cos (\alpha-\gamma)$ $=\frac{b^{2}-a^{2}-r^{2}}{2 a r}$ ist,

$$
d \gamma=\frac{b^{2}-a^{2}-r^{2}}{2 r^{2}} d \alpha
$$


Hiermit ergibt sich also

$$
d \varphi=d \psi+d \gamma=\frac{d \sigma}{p \cos \alpha}+\frac{b^{2}-a^{2}-r^{2}}{2 r^{2}} d \alpha
$$

und, mit Rücksicht auf

$$
\begin{gathered}
\cos \alpha=\frac{r^{2}-\frac{a^{2}-b^{2}}{2 a b} \text { und } r^{2}=a^{2}+b^{2}+2 a b \cos \alpha,}{d \sigma=\frac{p}{a b} \frac{r^{2}}{2} d \varphi-\frac{a^{2}+b^{2}}{2 a b} p d \varphi+\frac{a^{2}+a b \cos \alpha}{a^{2}+b^{2}+2 a b \cos \alpha} p \cos \alpha d \alpha .}
\end{gathered}
$$

Bei der Integration über eine geschlossene Figur fallen die beiden letzten Integrale fort, weil die Grenzen gleich werden. Das erste, $\frac{1}{2} \int r^{2} d \varphi$, ist aber dem Inhalt $F$ der umfahrenen Fläche gleich, und diese ist mithin der Abwicklung der Rolle bis auf einen konstanten Faktor gleich. Dieser Faktor $\frac{a b}{p}$ ist also der Wert der Noniuseinheit, d. h. die Fläche, welche $\sigma_{2}-\sigma_{1}=1$ entspricht.

Liegt der Pol innerhalb, so wird das zweite Integral nicht null, sondern es ist

$$
F=\frac{a b}{p}\left(\sigma_{2}-\sigma_{1}\right)+\left(a^{2}+b^{2}\right) \pi .
$$

Der Kreis mit dem Radius $\sqrt{a^{2}+b^{2}}$, dessen Fläche bei „Pol innen" zur abgelesenen Fläche hinzugefügt werden muB, und der vom Fahrstift bei senkrechter gegenseitiger Stellung von Fahrarm und Polarm beschrieben wird, heißt Ordinatenkreis. ${ }^{1}$ )

1) Fehlereinflüsse: Die Integrierrolle soll bei senkrechter Stellung von Polarm und Fahrarm die Scheibe bzw. Kugelkalotte im Mittelpunkt berühren.

Ist diese Bedingung nicht streng erfüllt, so ist bei außerhalb der Figur liegendem Pol der Fehler einflußlos. Wenn nämlich $c \cos \alpha+\Delta$ an Stelle von $c \cos \alpha$ tritt, d. h. der Rollenberührungspunkt, der einen Kreisbogen um den Drehpunkt des Fahrarms beschreibt, bei der Grundstellung $\alpha=90^{\circ}$ den Bogenabstand $\Delta$ statt 0 hat, so wird

$$
\begin{aligned}
d \sigma= & p^{\prime}(c \cos \alpha+\Delta) d \psi=p^{\prime}(c \cos \alpha+\Delta)(d \varphi-d \gamma) \\
=p^{\prime}\left[\frac{c}{a b} \frac{r^{2}}{2} d \varphi-c \frac{a^{2}+b^{2}}{2 a b} d \varphi+\Delta d \varphi\right. & \left.+(c \cos \alpha+\Delta) \frac{a^{2}+a b \cos \alpha}{a^{2}+b^{2}+2 a b \cos \alpha} d \alpha\right] .
\end{aligned}
$$

Bei Umfahrung einer geschlossenen Fläche bleibt also bei „Pol 
Die Genauigkeit der verschiedenen Planimeter ist durch Bestimmung des mittleren Umfahrungsfehlers ermittelt worden. ${ }^{1}$ ) Hierbei wurde der Flächeninhalt $F=n f$ gesetzt, indem mit $n$ die Anzahl der Umdrehungen der MeBrolle bezeichnet wurde, also $f$ der Flächenwert ist, welcher einer Umdrehung der MeBrolle entspricht. Es ergab sich für das

\section{Linearplanimeter}

Polarplanimeter

Präzisionsplanimeter (einf.)

"3

\section{freischwebendes Planimeter}

$$
\begin{array}{r}
d F=0,00081 f+0,00087 \sqrt{F f} \\
0,00126 f+0,00022 \sqrt{F f} \\
0,00056 f+0,00084 \sqrt{F f} \\
0,00069 f+0,00018 \sqrt{F f}
\end{array}
$$$$
0,00060 f+0,00026 \sqrt{F f}
$$

woraus die Überlegenheit der beiden zuletzt genannten Planimeter hervorgeht (man kann auch $\sqrt{F f}=\frac{F}{\sqrt{n}}$ einsetzen).

Das Polarplanimeter ist, wie es scheint, das einzige Plani-

auBerbalb" $\sigma_{2}-\sigma_{1}=p^{\prime} \frac{c}{a b} F$, indem die anderen Integrale fortfallen, wenn die Grenzen gleich werden. Für „Pol innerbalb" folgt $\sigma_{2}-\sigma_{1}$ $=p^{\prime}\left(\frac{c}{a b} F-c \frac{a^{2}+b^{2}}{a b} \pi+2 \Delta \pi \frac{c}{a b}\right)$, und das letzte Glied gibt daher den FeblereinfluB an.

Von den übrigen Bedingungen ist die wichtigste, daB die Rollenachse und der Fahrarm parallel sind. Ist dies nicht streng der Fall, sondern ein kleiner Winkel $\varepsilon$ rorhanden, so tritt $c \cos (\alpha+\varepsilon)$ an Stelle von $c \cos \alpha$ und es ist mit genügender Annäberung $d \sigma=p^{\prime}(c \cos \alpha$ $-c \varepsilon \sin \alpha) d(\varphi-\gamma)$. Dadurch tritt zu dem Flächenintegral noch ein Integral von der Form $\frac{p^{\prime} c \varepsilon}{2 a b} \int \sin \alpha d \varphi$ hinzu, das wegen

$$
\sin \alpha=\frac{1}{2 a b} \sqrt{4 a^{2} b^{2}-\left(r^{2}-a^{2}-b^{2}\right)}
$$

über eine Funktion $\Gamma(\boldsymbol{r})$ des Radiusvektors sich erstreckt. Die Anzahl der Rollenumdrehungen ist daher nicht der Fläche proportional, sondern auch vom Leitstrahl, also ron der Lage des Pols abhängig. Um daher das Planimeter in dieser Beziehung zu prüfen, ist eine Umfahrung der gegebenen Figur bei verschiedenen Polstellungen nötig. Ferner wird aus zwei Umfahrungen mit symmetrischer Polstellung dieser Fehler eliminiert (s. d. f.).

1) Osterreichische Zeitschrift für Berg- und Hüttenwesen, 1883. 
meter, das sich mit geringer Abänderung zur Flächenbestimmung auf einer nicht abwickelbaren Oberfläche, insbesondere auf der Kugelfläche verwenden läßt. Die hauptsächlichste Änderung besteht darin, daß sowobl der Polarm als der Fahrarm eine Art Knick (oder verstellbares Horizontalgelenk $j$ und $j^{\prime}$ Fig. 58 ) haben, so dab der Pol- und Fahrstift einerseits, die Rolle andererseits die Kugel berühren können. An Stelle der Bedingung, daß die

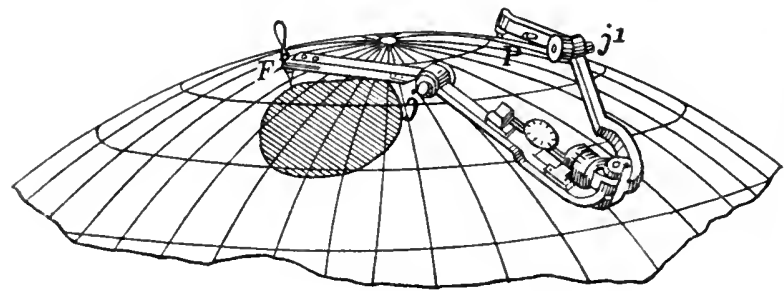

Fig. 58.

Rollenachse dem Fahrarm parallel ist, tritt die andere, dab ihre Richtung immer durch den Fahrstift geht.

41. Kompensations-Polarplanimeter. Wenn die Achse der MeBrolle dem Fahrstab nicht genau parallel ist (Fig. 59), so entsteht ein Fehler, der sich aber durch zwei Umfahrungen der Figur bei symmetrischer Stellung des Pols zum Fahrstab elimi-
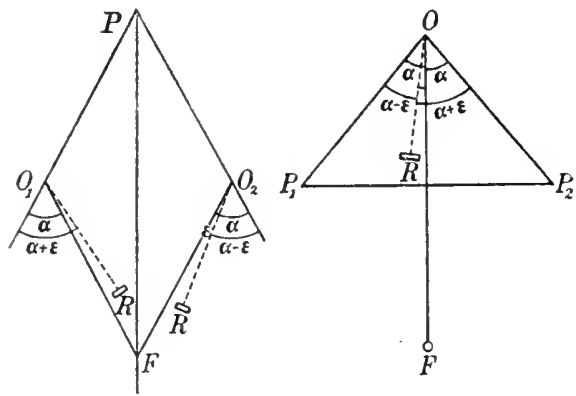

Fig. 59. nieren läßt. Dabei kann entweder die Verbindungslinie Pol-Fahrstift mit Beibehaltung ein und derselben Polstellung oder der Fahrarm bei zwei verschiedenen Polstellungen Symmetrieachse sein. Zu diesem Zwecke ist das Kompensationspolarplanimeter konstruiert, welches ein Durchschlagen des Fahrarms gestattet, $d$. h. der Fahrarm kann unter dem Polarm hindurchgeführt werden (Fig. 60). Es ist damit zugleich der Vorteil verbunden, daß das Instrument auseinander genommen werden und seine beiden Arme für sich aufbewahrt werden können. Die Verbindung zwiscben beiden Armen wird durch 
sehene Seite $B C$ den an ihr verschiebbaren und festzustellenden Fahrstift $F$ trägt. Es sei noch erwähnt, daß das freie Ende des Polarms auf einer Stützrolle aufliegt und dab die den Fabrstift tragende Parallelogrammseite an beiden Enden Führungsgriffe besitzt. Ein Winkelstück $B R C$, das an dieser Seite befestigt ist, hält in seinem Scheitelpunkte den die MeBrolle $R$ tragenden Schlitten, so daB bei einer Bewegung des Parallelogramms bzw. des Fahrstifts die MeBrolle längs des Polarms hin- und hergleitet.

Wird der Fabrstift durch ein Linienelement $F F_{2}$ geführt, so können wir diese Bewegung in zwei Teilen $F F_{1}$ und $F_{1} F_{2}$ vollzogen denken (vgl. Fig. 57). Bei der ersten Bewegung beschreibe $F$ einen Kreisbogen um den Pol,

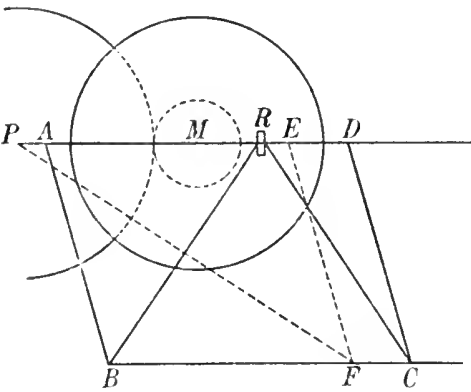

Fig. 61. dann bleibt das Parallelogramm unverändert und der Polarm überstreicht denselben Winkel $d \psi$, wie der Leitstrahl $r{ }^{1}$ ) Der zweite Teil der Bewegung wird durch die Bewegung des Parallelogramms hervorgebracht, wälırend der Polarm in Ruhe bleibt; der vom Leitstrahì überstrichene Winkel sei $d \gamma$, und $d \varphi=d \psi+d \gamma$ der der ganzen Bewegung $\mathrm{FF}_{2}$ entsprechende Winkel am Pol $P$. Wir bezeichnen die Entfernung vom Pol $P$ bis zum Schnittpunkt $E$ einer durch $F$ zu den Parallelogrammseiten $A B$ und $D C$ gelegten Parallelen mit $b$ und setzen die Länge der Parallelen $F E=A B=D C=a$. Es sei der Winkel des Parallelogramms bei $A$ oder auch $F E D=\alpha$. Nur bei der Führung des Fahrstifts längs $F F_{1}=r d \psi$ findet eine Drehung der Scheibe statt, indem die kleine Friktionsscheibe mit dem Radius $l$ sich um einen Winkel $d \lambda$ dreht, der durch ihr Rollen an dem Polsektor bestimmt ist. Ist $P$ der Radius des Polsektors, so rollt die Scheibe bei der Drehung des Polarms um $d \psi$ längs des Bogens $P d \psi$ und es ist $L d \lambda=P d \psi$. Die große Scheibe dreht sich um denselben

1) Es seien $F F^{\prime}$ zwei benachbarte Punkte und $P F=P F^{\prime}$ und es werde $E$ bei der Bewegung von $F$ nach $E^{\prime}$ übergeführt. Nun ist $P E=P A+A E=P A+B F$ konstant, also $P E^{\prime}=P E$. Da auch $E F^{\prime}=E^{\prime} F^{\prime}=A B=D C$ ist, so erhalten wir Winkel $E P F=E^{\prime} P F^{\prime}$. 
Winkel $d \lambda=\frac{P}{L} d \psi$. Wenn die Mebrolle in der Grundstellung des Instruments, in der das Parallelogramm zu einem Rechteck wird $\left(\alpha=90^{\circ}\right)$, den Abstand $\delta$ rom Mittelpunkte der Scheibe hat, so hat sie bei einer anderen Stellung des Parallelogramms den Abstand $\delta+a \cos \alpha$ (da ja ihre Entfernung rom FuBpunkt eines ron $F$ auf $A B$ gefällten Perpendikels konstant bleibt und dieser FuBpunkt sich um $a \cos \alpha$ rersehoben hat). Daher ist die Abwicklung der MeBrolle

$$
(\delta+a \cos \alpha) d \lambda=(\delta+a \cos \alpha) \underset{L}{P} d \psi=d \sigma .
$$

Wenn wir $d \psi=d \varphi-d \gamma$ einsetzen, so wird mit Rücksicht auf $\cos a=\frac{r^{2}-a^{2}-b^{2}}{2 a b}$

$d \sigma=\frac{P}{L}\left(\frac{1}{b} \frac{r^{2}}{2} d \varphi-\left[\frac{a^{2}}{2 b}+\frac{b}{2}\right] d \varphi+\delta d \varphi-(\delta+a \cos \alpha) d \gamma\right)$.

Um $d \gamma$ durch $d$ c auszudrücken, gehen wir auf Fig. 57 zurück, indem $P O$ und $P O_{1}$ die beiden Lagen des Polarms sind, und der Winkel $\gamma$ von der Lage $P O_{1}$ ab gerechnet wird. Dann ist

$$
a^{2}=b^{2}+r^{2}-2 b r \cos \gamma
$$

also $d \gamma=\frac{b \cos \gamma-r}{b r \sin \gamma} d r$ und wegen $r d r=-a b \sin \alpha d \alpha$

Es ist ferner

$$
-d \gamma=\frac{b \cos \gamma-r}{r^{2} \sin \gamma} a \sin \left(c d c^{1}\right)
$$

und

$$
a \sin \omega=r \sin \gamma
$$

oder

$$
r \cos \gamma=b+a \cos \alpha
$$

und

$$
b \cos \gamma-r=\frac{b^{2}-r^{2}+a b \cos \alpha}{r}
$$

also

$$
r^{2}=a^{2}+b^{2}+2 a b \cos \alpha
$$

$$
d \gamma=\frac{a^{2}+a b \cos \alpha}{a^{2}+b^{2}+2 a b \cos \alpha} d \alpha .
$$

1) Die Herleitung ist absichtlich etwas verschieden von derjenigen auf S. 107, die ebenso gut angewandt werden konnte.

Galle: Mathematische Instrumente 
Denken wir uns diesen Ausdruck eingesetzt und integriert, so verschwinden für eine geschlossene Figur alle Integrale mit Ausnahme des ersten, welches den Flächeninhalt gibt, nämlich

$$
\sigma_{2}-\sigma_{1}=\frac{P}{L b} \int \frac{r^{2}}{2} d \varphi
$$

Das Panintegrimeter von Kohlmorgen, das auch die Messung von Kurvenlängen umfaßt, bezweckt in erster Linie die Ausmessung von Flächenstücken insbesondere bei Wasserprofilen, meteorologischen Aufzeichnungen u. dgl. Dieser besondere Zweck kommt nur insofern in Betracht, als die Abszissen bei der Stationierung der FluBprofile und bei den nach der Zeit angestellten

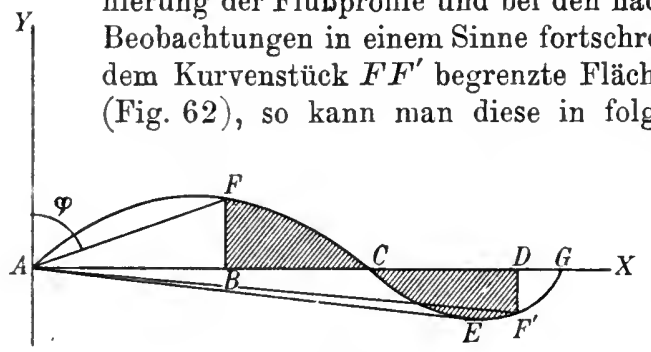

Fig. 62.

Wir unterscheiden:

1. die vom Leitstrahl überstrichene Fläche mit der Anfangslage $A F$ und der Endlage $A E$, die positiv gerechnet wird, insofern der Leitstrahl sich im Sinne des Uhrzeigers um $A$ dreht, 2. die vom Leitstrahl von der Anfangslage $A E$ bis zur Endlage $A F^{\prime}$ überstrichene Fläche, die negativ gerechnet wird, weil der Leitstrahl entgegengesetzt der Uhrzeigerbewegung sich dreht, 3. die von der Ordinate $y_{1}$ und Abszisse $x_{1}$ des Anfangspunktes $F$ begrenzte Dreiecksfläche $A B F$, die positiv $\frac{x_{1} y_{1}}{2}$ ist, und 4 . die von der Ordinate $y_{2}$ und der Abszisse $x_{2}$ des Endpunktes $F^{\prime}$ begrenzte Dreiecksfläche $\frac{x_{2} y_{2}}{2}$, die negativ gezählt wird, weil $y_{2}$ negativ ist. Wir erhalten im ganzen für die betrachtete (schraffierte) Fläche

$$
\begin{gathered}
\left(A C F+A C E-A E F^{\prime}\right)-\left(A B F-A D F^{\prime}\right) \\
=\frac{1}{2} \int \varrho^{2} d \varphi-\frac{x_{1} y_{1}}{2}+\frac{x_{2} y_{2}}{2}=\frac{1}{2} \int_{\varphi_{1}}^{\varphi_{2}} \varrho^{2} d \varphi+\frac{1}{2}\left(x_{2} y_{2}-x_{1} y_{1}\right)=\int_{x_{1}}^{x_{2}} y d x .
\end{gathered}
$$


Wir erhalten das auch leicht allgemein, wenn wir ron dem Ausdruck des Flächenelements

ausgehen und

$$
d F=\frac{1}{2}(y d x-x d y)=y d x-d\left(\frac{x y}{2}\right)
$$

einführen. Es ist

$$
y=\rho \cos \varphi, \quad x=\varrho \sin \varphi
$$

also

$$
\begin{aligned}
& y d x=\rho^{2} \cos ^{2} \varphi d \varphi+\rho \sin \varphi \cos \varphi d \varphi \\
& x d y=-\varrho^{2} \sin ^{2} \varphi d \varphi+\varphi \sin \varphi \cos \varphi d \varphi
\end{aligned}
$$

daher

$$
y d x-d\left(\frac{x y}{2}\right)=\frac{1}{2} \rho^{2} d \varphi
$$

$$
\int_{x_{1}}^{x_{2}} y d x=\frac{x y}{2} x_{x_{1} y_{1}}^{x_{2} / 2}+\frac{1}{2} \int_{\varphi_{1}}^{\varphi_{2}} \rho^{2} d \varphi \text {. }
$$

Das Panintegrimeter miBt nun $\int_{\varphi_{1}}^{\varphi_{2}} \rho^{2} d \varphi$. Es besteht im wesentlichen (Fig. 63) aus vier Stäben $\stackrel{\varphi_{1}}{M O} O=O F=a, A P=B P=\frac{a}{2}$, die in $O, A$ und $B$ durch Gelenke rerbunden sind, wobei $A$ und $B$ die Mitten ron $O M$ und $O F$ sind. In $F$ befindet sich der Fahrstift, in $\boldsymbol{P}$ der Pol, so da $B$ die gedachte Verbindung $P F=\rho$ den Leitstrahl vorstellt. Es ist nun noch ein Arm $P I=\lambda$ senkrecht zu $A P$, in fester Verbindung damit, angebracht, der also auch zu $O F$ senkrecht steht. so $\mathrm{daB} \varrho^{2}=2 a \lambda$ und $\frac{1}{2} \varrho^{2} d \varphi=a \lambda d \varphi$ ist. Die Verschiebung ron $L$ auf $M_{0}$ dem Arm $O F$, der in einem Schlitz den bei $L$ angebrachten Stift führt, äberträgt sich durch einen Seilzug,

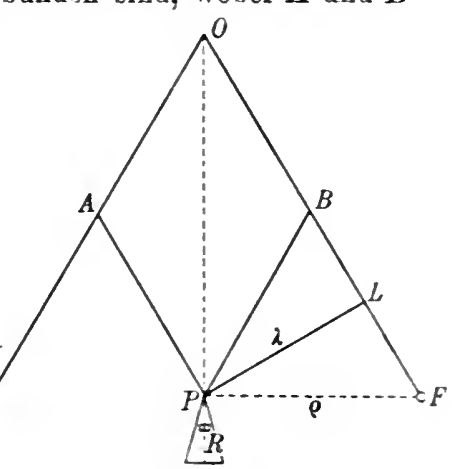

Fig. 63. der über $B O P$ geht, auf eine Rolle $R$, die sich infolge dessen in der Richtung $O P$ um $\lambda^{\prime}=\frac{\lambda}{n}$ rerschiebt. Um die Abwälzung der Rolle von der Cnterlage unabbängig zu machen, liegt sie auf 
einer Kegelfläche auf und verschiebt sich auf ihr längs einer horizontal liegenden Mantellinie. Wenn $P F$ sich um den Winkel $d \varphi$ um $P$ dreht, so dreht sich auch $O R$ um $P$ um $d \varphi$, denn das Dreieck FPO ist vor und nach der Drehung ein rechtwinkliges. ${ }^{1}$ ) Die Kegelfäche rollt bei dieser Drehung von $O R$ aut ihrem Grundkreise, während die Kegelspitze die Höhe $z$ über der Zeichenebene hat. Ist $s$ die Länge der Mantellinie, so ist der Weg, den der Berührungspunkt des Grundkreises beschreibt $\sqrt{s^{2}-z^{2}} \cdot d \varphi$. Ist ferner $\alpha$ der halbe Öffnungswinkel des Kegels, so dreht er sich um seine Achse um einen Winkel $d \psi$, der durch $s \sin \alpha d \psi$ $=\sqrt{s^{2}-z^{2}} d \varphi$ berechnet wird. Die Rolle berührt den Kegel in der Entfernung $\lambda^{\prime}$ von der Kegelspitze, mithin ist $d \sigma=\lambda^{\prime} \sin \alpha d \psi$ ihre Abwicklung. Man erhält schlieBlich:

$$
\frac{1}{2} \varrho^{2} d \varphi=a \lambda d \varphi=a n \lambda^{\prime} \cdot \frac{s \sin \alpha}{\sqrt{s^{2}-z^{2}}} \frac{d \sigma}{\lambda^{\prime} \sin \alpha}
$$

oder wenn $\frac{a n s}{\sqrt{s^{2}-z^{2}}}=k$ gesetzt wird, wo $k$ eine Konstante ist, so ergibt sich

$$
\frac{1}{2} \int \varrho^{2} d \varphi=k d \sigma
$$

Für die anderen Zwecke, denen das Instrument noch dient, sind in $M$ und am Stabe $O F$ ebenfalls Rollen angebracht.

Wir gehen nicht auf das Bryandsche Planimeter für Diagramme mit veränderlichem Maßstab ein, da seine Theorie nichts wesentlich neues bietet und die praktische Herstellung der dafür nötigen Führungskurven einige Schwierigkeit macht.

Finige Andeutungen über ein Planimeter von Adolf Schmidt in Potsdam zur Ausmessung registrierter Kurven inögen noch Platz finden. Der ausgeführte Apparat ist speziell für Aufzeichnungen der magnetischen Elemente am Potsdamer Observatorium eingerichtet, läßt sich aber leicht für andere Zwecke abändern. Die Kurven werden auf einem Papierbogen erhalten, der um einen Zylinder gewickelt ist, der sich durch ein Uhrwerk in einem bestimmten Zeitintervall, z. B. in einem Tage einmal um seine Achse dreht. Die in gleichen Abständen gezogenen Linien längs der Erzeugenden des Zylinders teilen dieses Intervall z. B. in Stunden.

1) $P F^{2}+P O^{2}=P B^{2}+B F^{2}+P B^{2}+B O^{2}=a^{2}$. 
Eine Linie senkrecht zu den Erzeugenden etwa am Ende des Zylinders (der Grundkreis) stellt die Nulllinie oder Abszissenachse für die Kurven dar. Es handelt sich nun im vorliegenden Falle darum, den mittleren Ordinatenwert $y_{m}$ für ein bestimmtes Abszissenintervall $x_{2}-x_{1}$, also z. B. für ein Stundenintervall zu ermitteln, so daB $y_{m}\left(x_{2}-x_{1}\right)=\int_{x_{1}}^{x_{2}} y d x$ oder $y_{m}=\frac{1}{x_{2}-x_{1}} \int_{x_{1}}^{x_{2}} y d x$ ist.

$\mathrm{Zu}$ diesem $\mathrm{Z}$ wecke ist ein Wagen auf einer dem horizontal gelegten Zylinder parallelen Laufschiene angeordnet, der den Fahrstift trägt, den man auf den Kurvenpunkt einstellt. Er ist durch eine ebenfalls dem Zylinder parallele Stange mit einer MeBrolle verbunden, die bei einer Verschiebung des Stifts in der Ordinatenrichtung (durch Bewegung des Wagens) längs dem Durchmesser einer horizontalen Kreisscheibe gleitet. Die Achse der Rolle ist dem Durchmesser, ebenso der Zylinderachse, parallel. Diese horizontale Scheibe und der Zylinder werden durch eine Kurbel in gleichzeitige Undrehung versetzt, so daB vollständige Umdrehungen beider zusammenfallen (oder wie im vorliegenden Falle proportional sind). Die Lage der Rolle, bei der ihr Berührungspunkt im Nittelpunkt der Scheibe liegt, entspricht der Stellung $y=0$ des Fahrstifts. Dreht sich die Scheibe um den Winkel $d \varphi=p d x$, wo $p=1$ (oder ein konstanter Proportionalitätsfaktor) ist, und hat der auf den Kurvenpunkt eingestellte Fahrstift die Ordinate $y$, so hat auch die Rolle den Abstand $y$ vom Mittelpunkt der Scheibe und wickelt $d \sigma=p y d x$ ab. Für die Drehung des Zylinders um $x_{2}-x_{1}$ und die entsprechende Drehung $p\left(x_{2}-x_{1}\right)$ der Scheibe erhält man

$$
\sigma_{2}-\sigma_{1}=\int_{x_{1}}^{x_{2}} y d x \text { und } y_{m}=\frac{\sigma_{2}-\sigma_{1}}{x_{2}-x_{1}}
$$

Aus praktischen Gründen ist die Nullstellung des Fahrstifts in der Mitte des Zylinders gewählt, so dab $y^{\prime}=y+a$ gesetzt werden kann, ferner ist eine Skala längs des Zylinders fest angebracht, durch die man die Stellung des Fahrstifts kontrolliert.

Namentlich in Amerika werden zahlreiche Instrumente angewendet, die auf einer rotierenden, kreisförmigen Papierscheibe Diagramme aufzeichnen, die demnach auf ein Polarkoordinatensystem bezogen sind. Die Scheibe dreht sich gleichmäBig z. B. in 
24 Stunden herum, während der Schreibstift sich entweder radial oder in einem Bogen vom Mittelpunkt weg oder auf ihn zu bewegt. Zur Auswertung dieser Diagramıe ist das Durand-Amslersche Radialplanimeter konstruiert worden. ${ }^{1}$ ) Es besteht aus einem zylinderförmigem Gewicht, das zentrisch über den Nittelpunkt der Scheibe gestellt wird. Durch dasselbe sind zwei parallele Führungsstangen gelegt, an denen sich der Rahmen bewegt, der den Fahrstift und in gleicher Entfernung vom Scheibenmittelpunkt die Meßrolle trägt. Die Verbindungslinie des Mittelpunktes der Scheibe mit dem Fahrstift stellt in jedem Augenblick den Leitstrahl dar, dem die Rollenachse parallel liegt. Wenn der Leitstrahl den Winkel $\varphi_{2}-\varphi_{1}$ überstreicht, so ist die Rollenablesung $r\left(\varphi_{2}-\varphi_{1}\right)$ proportional, wenn $r$ den mittleren Wert der Länge des Leitstrahls bedeutet. $\left.{ }^{2}\right)$ Dividiert man also durch $\varphi_{2}-\varphi_{1}$, so erhält man diesen Mittelwert, der die mittlere Ordinate bei einem in rechtwinkligen Koordinaten dargestellten Diagramm bedeuten würde. Die Genauigkeit des Instrumentes entspricht nach Amsler und Hammer derjenigen des einfachen Polarplanimeters.

43. Momentenplanimeter. Das Momentenplanimeter von Amsler besteht hauptsächlich aus einem Wagen, der auf zwei Rädern und dem Fahrstift ruht. Die beiden Räder laufen hintereinander in der Nut (Rinne) eines Lineals, so daB der ganze Wagen parallel mit dem Lineal geradlinig verschoben werden kann (Fig. 64). Dieser Wagen trägt ein horizontal licgendes Zahnrad $A$, das sich um Fig. 64. einen senkrecht in seinem Mittelpunkte $O$ stehenden Zapfen dreht. Ein verlängerter Radius dieses Zahnrades ist der Fahrarm $O F$, der den Fahrstift $F$ trägt. In das große Zahnrad $A$ greifen zwei kleinere Zahnräder $B$ und $C$ ein, die ebenfalls horizontal liegend auf dem Wagen so angebracht sind, daß die Verbindungslinie der

1) Zeitschr. f. Instrumentenk. 31, 213 u. 214 (Hammer, Zusatz zur Mitteilung von Amsler).

2) Gleichen Zentriwinkeln $\Delta \varphi$ entsprechen Längenelemente der registrierten Kurve $r_{1} \Delta \varphi, r_{2} \Delta \varphi, \ldots r_{n} \Delta \varphi$. Die Rolle summiert $r_{1} \Delta \varphi+r_{2} \Delta \varphi+\cdots+r_{n} \Delta \varphi=\frac{r_{1}+r_{2}+\cdots+r_{n}}{n} n \Delta \varphi=r\left(\varphi_{2}-\varphi_{1}\right)$. 
Mittelpunkte der drei Räder senkrecht zum Lineal liegt. Der Radius des Rades $B$ beträgt die Hälfte des Radius des Zahnrades $A$, der des Rades $C$ ein Drittel desselben. Dies wird bei gleicher Gröbe der Räder $B$ und $C$ dadurch erreicht, da $B$ das groBe Rad $A$ aus zwei Teilen mit rerschieden großen, im Verbältnis $2: 3$ stehenden Radien besteht und der Sebtor mit dem gröBeren Radius in $C$, der andere in $B$ eingreift. Die Lage der drei Räder ist so gewählt, daB das Spiel des Fahrarms möglichst wenig beschränkt und andrerseits das Umschlagen verhindert wird, und da $B$ der Wagen .sich nicht einmal rechts, ein andres Mal links rom Fahrstift befindet.

Der Fahrarm trägt eine auf der Zeichenebene aufliegende MeBrolle $m_{1}$, deren Achse ihm parallel ist. Ebenso tragen die beiden kleinen Räder MeBrollen, deren Achsen in der Ebene der Räder liegen und radial gerichtet sind. Diese drei Rollen helfen den Wagen tragen; damit sie aber nur mit einem geringen Druck auf der Zeichenebene aufliegen, wird der Wagen durch eine über das Lineal hinwegreichende Stange mit einem Gegengewicht entlastet.

Der Apparat dient zur Bestimmung des Flächeninhalts, des statischen Moments und des Trägheitsmomentes einer Figur in bezug auf irgend eine Momentenachse in der Ebene der Figur. Betrachten wir diese als $x$-Achse, so muB der Mittelpunkt $O$ des Rades $A$ auf ihr liegen und das Lineal ihr parallel sein. Es wird dies leicht bewerkstelligt, indem zwei auf das Lineal senkrecht zu ihm aufzusetzende Hilfsarmè mit ihren Spitzen auf die $x$-Achse gesetzt werden.

Denkt man sich zunächst den Fahrarm, also auch die Achse der daran befindlichen Laufrolle $m_{1}$ in die Richtung $\cdot \operatorname{der} x$-Achse gestellt, so ist die Achse der Laufrolle $m_{3}$ des Rades $C$ ebenfalls parallel der $x$-Achse oder der Richtung des Lineals, die Achse der Laufrolle $m_{2}$ des Rades $B$ aber senkrecht zum Lineal gerichtet. Bei einer Bewegung des Wagens (in der Richtung der $x$-Achse) würde dann nur die Rolle $m_{2}$ den zurückgelegten $W^{\text {eg messen, }}$ während die beiden anderen Rollen keine Drehung erlitten.

Wenn man jetzt bei stillstehendem Wagen den Fahrarm um einen Winkel a gegen die $x$-Achse dreht, so dreht sich das Rad $A$ um denselben Winkel und infolgedessen $C$ um den dreifachen, $B$ um den doppelten Winkel. Die Achse der Rolle $m_{1}$ wird dann den Winkel $\alpha$, die der Rolle $m_{3}$ den Winkel $3 \alpha$, die der Rolle $m_{3}$ den Winkel $90^{\circ}-2 \alpha$ mit der $x$-Achse bilden, da letztere rorher 
den Winkel $90^{\circ}$ mit ihr einschlob und die Räder $B$ und $C$ sich in entgegengesetztem Sinne drehen.

Ist a die Länge des Fahrarms, also $y=a \sin \alpha$ die Ordinate des Fahrstifts, so erhält man die umfahrene Fläche

$$
\int y d x=\int a \sin \alpha d x
$$

Die Rolle $m_{1}$ am Planimeterarm wickelt aber $d \sigma=\sin \alpha d x \mathrm{ab}$, man erhält daher an einer an ihr angebrachten Ablesevorrichtung

$$
\sigma_{2}-\sigma_{1}=\frac{1}{a} \int y d x
$$

und damit den Flächeninhalt $F=a\left(\sigma_{2}-\sigma_{1}\right)$.

Liest man an der Rolle $m_{2}$ des Rades $B$ ab statt an der Rolle des Fahrarms, so hat man nur $90^{\circ}-2 \alpha$ statt $\alpha$ einzusetzen und erhält

$$
\int a \cos 2 \alpha d x=a \int d x-2 \int a \sin ^{2} \alpha d x \text {. }
$$

Das erste Integral verschwindet, wenn man eine geschlossene Figur umfährt, also denselben Anfangs- und Endwert von $x$ erhält. Setzt man im zweiten Integral

ein, so erhält man

$$
\sin ^{2} \alpha=\frac{y^{2}}{a^{2}}
$$

$$
2 \int \frac{y^{2}}{a} d x=\frac{2}{a} \int y d F,
$$

wo $d F=y d x$ das Flächenelement ist. Nun ist das statische Moment eines Flächenteilchens $d x d y$ in bezug auf die $x$-Achse $y d x d y$, also das der ganzen Fläche

$$
\iint y d x d y=\frac{1}{2} \int y^{2} d x=M_{2} .
$$

Die Ablesung ${ }^{1}$ ) der Rolle $m_{2}$ gibt also

$$
\sigma_{2}{ }^{\prime}-\sigma_{1}{ }^{\prime}=\frac{4}{a} M_{2} \text {. }
$$

Ist $y_{0}$ die Ordinate des Schwerpunkts der Fläche, also

$$
y_{0} F=\int \dot{y} d F
$$

1) Die Teilung der Rolle wird in dem Sinne gewählt, daß die Differenz $\sigma_{2}-\sigma_{1}$ das positive Integral liefert. 
sind die MeBrollen eingefügt, die die Kugeln stets berühren. Diese Rahmen drehen sich um vertikale Achsen und sind durch Zahnräder in der Weise in Verbindung, daß bei einer Drehung des mit dem mittelsten Rahmen verbundenen Fahrarms um einen Winkel $\alpha$ aus der Normalstellung (Fahrarm senkrecht zur Rollenachse) die Achse der (in der Figur 65 rechts liegenden) Momentenrolle den Winkel $90^{\circ} \pm 2 \alpha$ mit derselben Anfangsrichtung bildet, die (links liegende) Trägheitsmomentenrolle den Winkel $3 \alpha$. Durch die Verwendung der Kugeln (an Stelle der Scheiben des vorigen Apparates) wird ein Gleiten der MeBrollen verhindert. Der Fahrstab kann um $40^{\circ}$ nach beiden Seiten aus der Normalstellung gedreht werden, so daß Flächen von etwa $50 \mathrm{~cm}$ Breite und beliebiger Längenausdehnung umfahren werden können. Die Theorie läBt sich unmittelbar von dem vorigen Apparat übertragen.

Einen anderen Apparat zur Bestimmung beliebiger Momente durch Parallelabschieben hat J. Schnöckel ${ }^{1}$ ) konstruiert. Ferner kommen wir bei den Integraphen auf diese Bestimmungen zurück.

44. Schneidenplanimeter und Abarten. Das in der Ausführung und dem Grundgedanken einfachste Planimeter ist das von dem dänischen Kapitän Prytz erfundene und in Tekniske Forenings Tidsskrift 1886 beschriebene Stangenplanimeter (Stangplanimeter, Beilschneidenplanimeter, Hatchet-Planimeter). ${ }^{2}$ )

Das Instrument besteht aus einer Stange, die an beiden Enden rechtwinklig in derselben Vertikalebene umgebogen ist(Fig. 66). Das

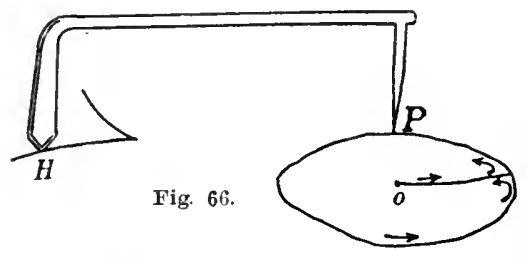
eine Ende ist zugespitzt, das andre trägt eine kleine, stark gekrümmte Schneide (Messerchen, Beilschneide), deren Ebene durch die Spitze geht. Man hält die Spitze mit zwei Fingern und führt sie in senkrechter Lage um die geschlossene Kurve herum, wobei das Messerchen nachgezogen wird. Kehrt man mit der Spitze wieder zum Anfangspunkt zurück, so ist das Messerchen im allgemeinen nicht in seinen Ausgangspunkt gelangt Hat man nun vor und nach der Umfahrung durch einen Druck auf die Schneide einen

1) S. Zeitschr. f. Vermessungswesen 24, S. 245, 1904.

2) Vgl. Prytz, Tidsskrift for Opmaalings- og Matrikulvaesen 1895, $383-392$ und die Beschreibung in der Broschüre des Fabrikanten Cornelius Knudsen in Kopenhagen 1894. 
Eindruck ins Papier gemacht, so ist, wenn $c$ der Abstand dieser beiden Marken und $a$ die Armlänge (Entfernung : Spitze - Schneide) ist, das Produkt $a c$ in erster Näherung der Flächeninhalt der umfahrenen Figur. Die Theorie ist schwieriger als bei anderen Planimetern, aber von Bedeutung für die Anwendung des Instrumentes. Man gelangt außer in einfachen Fällen (Quadrat und Kreis), wie es scheint, zu keinem geschlossenen Ausdruck. (Vgl. C. Runge, ZS. f. Vermessungswesen $\mathbf{9 4}(1895)$ und Ha mann, ebenda 25 (1896).

Wir denken uns die Fläche $F$ in Elementardreiecke zerlegt, die sämtlich in einem Punkte $O$ im Innern ihre Spitze haben, während jeder Leitstrahl von $O$ nach einem Punkte des Umfangs eine gemeinschaftliche Seite zweier benachbarten Dreiecke bildet, und jedes Linienelement der Umgrenzungskurre die der Spitze $O$ gegenüberliegende Seite ist. Wenn wir dann jedes Elementardreieck vom Punkte $O$ ausgehend mit dem Stift des Planimeters umfahren, so wird jeder Leitstrahl zweimal in entgegengesetztem Sinne durchlaufen und die Cmfahrung endet schlieBlich wieder im Punkte $O$.

Es sei $O S S^{\prime}$ ein solches Elementardreieck (Fig. 68), $O$ der Anfangspunkt und $O X$ die Anfangsrichtung für Polarkoordinaten. Der Winkel des Leitstrahles $O S=r$

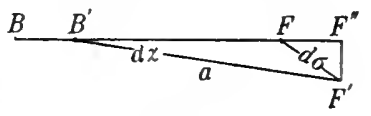

Fig. 67. mit $O X$ sei $\vartheta$, der von $O S^{\prime}=r+d r$ mit $O X$ eingeschlossene Winkel $\vartheta+d \vartheta$, so ist $d \vartheta$ der Winkel an der Spitze des Dreiecks.

Den Richtungswinkel des Planimeterarmes bezeichnen wir im Anfangspunkte $O$ bei Beginn der Umfahrung mit $\Phi$, am Ende mit $\Phi+d \Phi$. In $S$ sei er $\varphi$ und in $S^{\prime} \varphi+d \varphi$.

Wenn der Planimeterstift ein geradliniges Streckenelement (Fig. 6 6 ) $F F^{\prime}=d \sigma$ durchläuft, $\chi$ der Winkel des Planimeterarmes mit $F F^{\prime}$ im Punkte $F, \chi+d \chi$ in Punkte $F^{\prime}$ ist und $a$ die Länge des Armes bezeichnet, so werden wir die Bewegung des Planimeters in die beiden ihm allein möglichen Bewegungen zerlegen, von denen die eine in einer Verschiebung in der Richtung des Planimeterarmes von $B F$ nach $B^{\prime} F^{\prime \prime}$ besteht, während die andere den Übergang des Stiftes von $F^{\prime \prime}$ nach $F^{\prime}$ durch eine Drehung um den Berührungspunkt $B^{\prime}$ der Beilschneide hervorbringt. In dem Dreiecke $B^{\prime} F^{\prime} F^{\prime \prime}$ mit dem Winkel $d \%$ bei $B^{\prime}$ ist $F^{\prime} F^{\prime \prime}=a d \%$, mithin $\sin \chi d \sigma=a d \gamma$ oder

$$
\frac{d \chi}{\sin \chi}=\frac{d \sigma}{a} .
$$


Für eine endliche gerade Strecke $\sigma$ erhalten wir, wenn $\chi$ und $\chi$ die Winkel des Planimeters mit $\sigma$ am Anfang und Ende der Bewegung sind,

$$
x \quad \int_{\chi}^{\chi^{\prime}} d \chi{ }^{\sigma} \frac{\sigma}{a} \quad \text { oder } \lg \operatorname{tg} \frac{\chi^{\prime}}{2}-\lg \operatorname{tg} \frac{\chi}{2}=\frac{\sigma}{a},
$$

wofür man auch schreiben kann:

$$
\operatorname{tg} \frac{\chi^{\prime}}{2}=e^{\frac{\sigma}{a}} \operatorname{tg} \frac{\chi}{2} .
$$

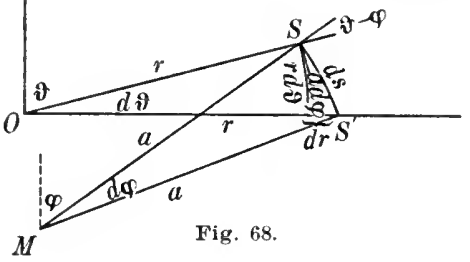

Diese Formel können wir sogleich anwenden für die Führung des Planimeterstifts längs der Geraden $O S=r$ (Fig. 68), wenn wir $\chi=\Phi-\vartheta, \chi^{\prime}=\varphi-\vartheta$ einsetzen:

$$
\operatorname{tg} \frac{\varphi-\vartheta}{2}=e^{\frac{r}{a}} \operatorname{tg} \frac{\Phi-\vartheta}{2} \text {. }
$$

Hiermit ist also $\varphi$ aus $\Phi$ erhalten. Wir lassen nun den Stift von $S$ nach $S^{\prime}$ fahren. Um die Änderung $d \varphi$ von $\varphi$ zu erhalten, stellen wir daher eine der Gleichung (1) entsprechende auf. Bezeichnen wir voriibergehend den Winkel, den $S S^{\prime}$ und das Kreisbogenelement $S S^{\prime \prime}=r d \vartheta$ untereinander einschließen, mit $\psi$, so ist

$$
\cos \psi d s=r d \vartheta, \quad \sin \psi d s=d r .
$$

Der Planimeterarm $M S$ schließt in $S$ mit $O S$ den Winkel $\vartheta-\varphi$ ein, also mit $S S^{\prime}$ den Winkel $\vartheta-\varphi+90^{\circ}-\psi$. Hier sind $\vartheta$ und $\psi$ als gegebene Winkel anzusehen, welche die Lage von $d s$ bestimmen. Wir wenden nun Gleichung (1) an und erhalten:

$$
\frac{d \varphi}{\cos (\vartheta-\varphi-\psi)}=\frac{d s}{a}
$$

oder

$$
a d \varphi=\cos (\vartheta-\varphi) \cos \psi d s+\sin (\vartheta-\varphi) \sin \psi d s
$$

und mit Rücksicht auf (4)

$$
a d \varphi=\cos (\varphi-\vartheta) r d \vartheta-\sin (\varphi-\vartheta) d r .
$$

Hiermit haben wir also $d \varphi$ durch $\varphi$ und die gegebenen Stücke er- 
halten. Wenn wir nun von $S^{\prime}$ nach $O$ zurückgehen, so könnten wir die der Gleichung (3) entsprechende Gleichung aufstellen:

$$
\operatorname{tg} \frac{\Phi+d \Phi-\vartheta-d \vartheta}{2}=e^{\frac{r+d r}{a}} \operatorname{tg} \frac{\varphi+d \varphi-\vartheta-d \vartheta}{2} .
$$

Wir erhalten aber $d \Phi$ bequemer, wenn wir Gleichung (3) sowohl nach $\Phi, \varphi$, wie nach $\vartheta$ und $r$ differenzieren, und für $d \varphi$ und $d r$ den bei der bisherigen Umfahrung erhaltenen Wert nach (5) einsetzen. Nach vorangegangener Logarithmierung der Gleichung (3) bekommen wir, wenn wir noch den andern Richtungssinn beachten:

$$
\frac{d(\Phi-\vartheta)}{\sin (\Phi-\vartheta)}=\frac{d r}{a}+\frac{d(\varphi-\vartheta)}{\sin (\varphi-\vartheta)} .
$$

Wir setzen $d \varphi$ aus (5) ein und finden

$$
\begin{gathered}
\frac{d(\Phi-\vartheta)}{\sin (\Phi-\vartheta)}=\operatorname{ctg}(\varphi-\vartheta) \frac{r}{a} d \vartheta-\frac{d \vartheta}{\sin (\varphi-\vartheta)} \\
=\frac{1}{2}\left[\frac{r}{a}\left(\operatorname{ctg} \frac{\varphi-\vartheta}{2}-\operatorname{tg} \frac{\varphi-\vartheta}{2}\right)-\left(\operatorname{ctg} \frac{\varphi-\frac{\vartheta}{2}}{2}+\operatorname{tg} \frac{\varphi-\vartheta}{2}\right)\right] d \vartheta \\
=\frac{1}{2}\left[\left(\frac{r}{a}-1\right) \operatorname{ctg} \frac{\varphi-\vartheta}{2}-\left(\frac{r}{a}+1\right) \operatorname{tg} \frac{\varphi-\vartheta}{2}\right] d \vartheta .
\end{gathered}
$$

Wenn wir jetzt aus der (7) entsprechenden Integralgleichung, die sich von $(6)$ nur durch Wegfall der DifferentialgröBen unterscheidet, die Werte für $(\varphi-\vartheta)$ einsetzen, so erhalten wir:

$$
=\frac{1}{2}\left[\left(\frac{r}{a}-1\right) e^{+\frac{r}{a}} \operatorname{ctg} \frac{\Phi-\vartheta}{\sin (\Phi-\vartheta)}-\left(\frac{r}{a}+1\right) e^{-\frac{r}{a}} \operatorname{tg} \frac{\Phi-\vartheta}{2}\right] d \vartheta
$$

und

$$
\begin{gathered}
d(\Phi-\vartheta)=\frac{1}{2}\left[\left(\frac{r}{a}-1\right) e^{+\frac{r}{a}}(1+\cos (\Phi-\vartheta))\right. \\
\left.-\left(\frac{r}{a}+1\right) e^{-\frac{r}{a}}(1-\cos (\Phi-\vartheta))\right] d \vartheta \\
=\frac{1}{2}\left[\frac{r}{a}\left(e^{+\frac{r}{a}}-e^{-\frac{r}{a}}\right)-\left(e^{+\frac{r}{a}}+e^{-\frac{r}{a}}\right)\right] d \vartheta \\
+\left[\frac{r}{a}\left(e^{+\frac{r}{a}}+e^{-\frac{r}{a}}\right)-\left(e^{+\frac{r}{a}}-e^{-\frac{r}{a}}\right)\right] \cos (\Phi-\vartheta) d \vartheta .
\end{gathered}
$$


Es ist

$$
\begin{aligned}
& e^{ \pm \frac{r}{a}}=1 \pm \frac{r}{a}+\frac{r^{2}}{2 a^{2}} \pm \frac{r^{3}}{6 a^{3}}+\frac{r^{4}}{24 a^{4}} \pm \frac{r^{5}}{120 a^{5}}+\frac{r^{6}}{720 a^{6}} \pm \cdots \\
& d(\Phi-\vartheta)=\left[\frac{r^{2}}{a^{2}}+\frac{r^{4}}{6 a^{4}}+\frac{r^{6}}{120 a^{6}} \cdots-1-\frac{r^{2}}{2 a^{2}}-\frac{r^{4}}{24 a^{4}}-\frac{r^{6}}{720 a^{6}}-\cdots\right] d \vartheta \\
& +\left[\frac{r}{a}+\frac{r^{3}}{2 a^{3}}+\frac{r^{5}}{24 a^{5}} \cdots-\frac{r}{a}-\frac{r^{3}}{6 a^{3}}-\frac{r^{5}}{120 a^{5}}-\cdots\right] \cos (\Phi-\vartheta) d \vartheta
\end{aligned}
$$

also

$$
d \Phi=\left[\frac{r^{2}}{2 a^{2}}+\frac{r^{4}}{8 a^{4}}+\frac{r^{6}}{144 a^{6}}+\cdots\right] d \vartheta+\left[\frac{r^{3}}{3 a^{3}}+\frac{r^{5}}{30 a^{5}}+\cdots\right] \cos (\Phi-\vartheta) d \vartheta
$$

Integriert man um die ganze Kurve, so sind die Grenzen $\vartheta=0$ und $\vartheta=2 \pi$ und wenn $\Phi$ in $\Phi+\Delta \Phi$ sich ändert, so daB das Planimeter in seiner Endstellung den Winkel $\Delta \Phi$ mit seiner Anfangsstellung einschließt, so ergibt sich:

$$
\begin{gathered}
a^{2} \Delta \Phi=\int_{0}^{2 \pi} \frac{r^{2}}{2} d \vartheta+\int_{0}^{2 \pi} \frac{r^{4} d \vartheta}{8 a^{2}}+\int_{0}^{2 \pi} \frac{r^{6} d \vartheta}{144 a^{4}}+\cdots \\
+\int_{0}^{2 \pi} \frac{r^{3}}{3 a} \cos (\Phi-\vartheta) d \vartheta+\int_{0}^{2 \pi} \frac{r^{5}}{30 a^{3}} \cos (\Phi-\vartheta) d \vartheta+\cdots
\end{gathered}
$$

Hier ist das erste Integral die Fläche, deren Inhalt zu bestimmen ist. Wenn die Fläche nicht groB ist und $r$ gegen die Länge des Planimeters klein bleibt, so gibt $a \cdot a \Delta \Phi$ den Flächeninhalt nahezu an. $a \Delta \Phi=\Delta S$ ist der Bogen, den der Berührungspunkt der Schneide um den Anfangspunkt $O$ beschrieben hat und der im Falle eines kleinen Bogens auch durch die Sehne, also die Entfernung der beiden Marken im Papier ersetzt werden kann:

$$
\begin{aligned}
a \Delta S= & F+\frac{1}{4 a^{2}} \int r^{2} d F+\frac{1}{72 a^{4}} \int r^{4} d F+\cdots \\
& +\frac{2}{3 a} \int r \cos (\Phi-\vartheta) d F+\cdots
\end{aligned}
$$

Das Integral im ersten Korrektionsglied stellt das Trägheitsmoment um eine durch $O$ gehende zur Fläche senkrechte Achse dar, es ist daher ein Minimum, wenn $O$ der Schwerpunkt der Fläche ist. Das folgende Glied ist jedenfalls kleiner als $\frac{F}{72}\left(\frac{R}{a}\right)^{4}$, wenn $R$ der gröBte 
vorkommende Leitstrahl ist. Das letzte der angeschriebenen Glieder hängt von der Anfangslage des Planimeters ab, und es ist ersichtlich, daB eine Änderung des Richtungswinkels um $180^{\circ}$, also Einführung von $180^{\circ}+\Phi$ statt $\Phi$, das Vorzeichen ändert. Wenn man also zwei Umfahrungen bei diametral entgegengesetzter Anfangslage des Planimeters macht, so fällt im Mittel aus beiden dieses Glied (und ebenso die folgenden) fort.

Iaffiotti fand für Flächen ron 4000 bis $8000 \mathrm{~mm}^{2}$ mittlere Fehler von 0,36 bis $1,16^{\circ}$ bei rechtläufiger, 0,28 bis $0,70 \%$ bei entgegengesetzter Führung des Fahrstiftes.

Bei einer etwas anderen Form „Goodmans Hatchet Planimeter", die eine Veränderung des Abstandes: Schneide-Fahrstift gestattet, ist die unmittelbare Ablesung ron Mittelordinaten (für Indikatordiagramme und dgl.) rorgesehen und eine Skala zur einfachen Berücksichtigung des Faktors $\left[1-\left(\frac{R}{2 a}\right)^{2}\right]$ angebracht. $\left.{ }^{1}\right)$

Andere Abänderungen bestehen in dem Ersatz der Schneide durch eine Rolle mit scharfem Rand, in seitlichen Stïtzen, welche die senkrechte Haltung des Planimeters gewährleisten usw.

Auf etwas verändertem Prinzipe beruhen einige andere Planimeter, unter denen wir dasjenige ron Fieguth in Danzig nennen. Die Rolle sitzt auf einem zum Planimeterarm nahezu senkrecht angebrachten Arm und verschiebt sich auf diesem. Die Verschiebung der Rolle auf diesem Querarm, die an einer Teilung abgelesen wird, gibt in die Fahrarmlänge multipliziert den Flächeninhalt.

Ähnlich ist das Planimeter von Lippincott, endlich auch dasjenige ron Weber-Kern. Bei letzterem ist der Fahrarm durch einen senkrecht stehenden Zylinder horizontal hindurchgesteckt, der auch den zum Fahrarm senkrechten Radarm trägt, an dessen Skala wiederum die Verschiebung eines auf ihm verschiebbaren scharfrandigen Rädchens abgelesen wird. Die Achse des Zylinders bewegt sich in unverändert senkrechter Lage längs der Nut eines auf der Zeichenebene aufliegenden Lineals. Der Fahrarm läBt sich durch den Zylinder hindurchziehen, so daB seine Länge

1) Es ist $F=a \Delta S-\begin{gathered}1 \\ 4 a^{2}\end{gathered} \int r^{2} d F$ odergenähert $F=a \Delta S-\frac{R^{2}}{4 a^{2}} F$ oder auch $F=a \Delta S\left(1-\frac{R^{2}}{4 a^{2}}\right)$. 
verändert werden kann. Die Theorie ist einfach, indem die Führungskurve als ein Kreis mit unendlich großem Radius angesehen werden kann. Das Planimeter kann nur zwei Arten von Bewegungen ausführen, erstens eine Drehung um den Schnittpunkt beider Achsen, bei der sich das Rad dreht, aber nicht auf seiner Achse verschiebt, zweitens eine Verschiebung parallel zur Richtung des Lineals, die wir als $x$-Achse denken. Ist der Winkel des Fahrarms von der Länge $a$ mit der $x$-Achse $\alpha$, so wird bei einer Verschiebung $d x$ das Rädchen erstlich eine nicht in Betracht kommende Abwicklung $\cos \alpha d x$ und zweitens eine Verschiebung $d v=\sin \alpha d x$ ausführen. Nun ist der Inhalt der zu umfahrenden Fläche

$$
F=\int y d x=\int a \sin \alpha d x=a \int d v=a\left(v_{2}-v_{1}\right) .
$$

Um beim Prytzschen Planimeter den Flächeninhalt aus $a^{2} \Delta \Phi$ zu berechnen (vgl. S. 126), besitzt man kein einfaches Hilfsmittel, den Winkel $\Delta \Phi$ zwischen der Anfangs- und Endlage der Stange zu messen. Deshalb hat Horst von Sanden das Instrument in der Weise abgeändert (Fig. 69), daß statt der Schneide zwei Rollen mit scharfem Rande angebracht werden, die auf einer zur Stange rechtwinkligen Achse in gleicher Entfernung von der Stange sitzen. Der Mittelpunkt $M$ zwischen beiden Kollen, also der Endpunkt der Stange, wird dann in der gleichen Weise wie die Schneide geführt. ${ }^{1}$ ) Die Berührungspunkte der Rollen beschreiben zwei Parallelkurven. Ist das vom Punkte $\boldsymbol{M}$ beschriebene Bogenelement $d s=r d \varphi$ und der Abstand der Rollen 2b, so werden die beiden Rollen Bogenelemente $d \sigma_{1}=(r+b) d \varphi$ und $d \sigma_{2}=(r-b) d \varphi$ beschreiben. Damit folgt $d \sigma_{1}-d \sigma_{2}=2 b d \varphi$, also

$$
\int\left(d \sigma_{1}-d \sigma_{2}\right)=2 b \Delta \Phi
$$

Nun ist die Differenz $d \sigma_{1}-d \sigma_{2}$ der relativen Verdrehung der Rollen gegeneinander gleich. Um sie ablesen zu können, ist jede Rolle mit einer Trommel in der Mitte der Achse starr verbunden. Die eine Trommel trägt eine Teilung, die andere zwei um $180^{\circ}$

1) Bei völliger Gleichheit der Rollenradien wird er bei bloßer Drehung der Stange an derselben Stelle bleiben, da dann beide Räder in verschiedener Richtung denselben Kreisbogen durchlaufen. Inwieweit dies praktisch erreicht ist, wird von dem Verf. in Zeitschr. für Mathematik u. Physik Bd.59, S. 314 nicht erwähnt. - Das Instrument ist wohlfeiler (54 M.) als andere Instrumente gleicher Genauigkeit. 
auseinanderstehende Nonien. An dem gerade sichtbaren Nonius wird die Verdrehung abgelesen, die mit einer Fonstanten multipliziert den Flächeninhalt angibt. Die Regeln für die Handhabung des Apparates schlieBen sich an die Theorie des Stangenplanimeters an. Bei dem ausgeführten Apparat ist der Rollenabstand $150 \mathrm{~mm}$, der Rollendurchmesser $40 \mathrm{~mm}$, die Stangenlänge $a$ ist zwischen 50 und $192 \mathrm{~mm}$ verstellbar, die Trommel ist in 100 Teile geteilt, ron denen Zehntel geschätzt werden. Die Genauigkeit ergab sich bei Umfahrung eines Kreises von $10 \mathrm{~mm}$ Radius $\left(0,7^{0}{ }_{0}\right.$ bei $a=191$, $0,4 \%$ bei $a=101$ ).

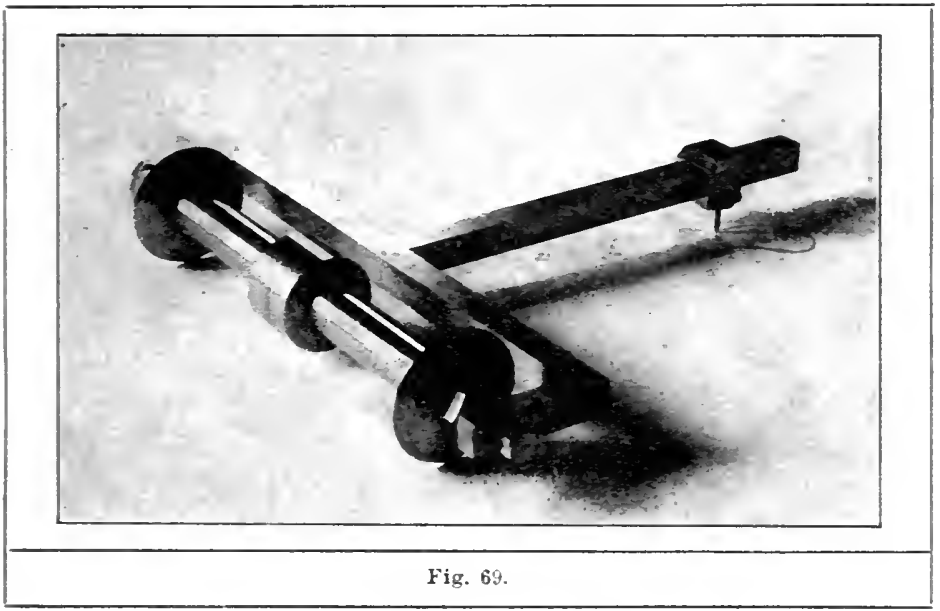

Ebenfalls zwei srmmetrisch an der Stange angebrachte Schneiderollen besitzt das Präzisions-Stangenplanimeter ron Th. Pregél (vgl. Zeitschr. f. Instrumentenk. 28, 373 und 31, 3̈73).

Das optische Planimeter ron J. Schnöckel unterscheidet sich hauptsächlich dadurch ron anderen, daB es keinen Fahrstift hat. Vielmehr befindet sich über der scharf kantigen Rolle, die am Ende eines langgestreckten rechteckigen Rahmens angebracht ist, ein senkrecht stehender Spiegel, dessen Ebene die Rollenachse und den Berühruugspunkt der Rolle enthält. Das Instrument dient hauptsächlich zur Ausmessung von Registrierstreifen und Ermittlung der mittleren Ordinate. Zu diesem Zwecke wird ein rorn befindlicher Index an den Anfangspunkt der Kurve gelegt, auf einem 
untergelegten Blatte Papier eine Gerade rückwärts in derselben Entfernung vom Rollenauflagepunkte parallel den Ordinaten der Kurve gezogen. Das Instrument selbst ist in seiner Längsrichtung parallel der Abszissenachse aufgestellt, so daß ein in dieser Richtung gespannter Faden die auf dem Papier gezogene Gerade $l$ in einem in bezug auf die Rolle symmetrisch zum Anfangspunkt gelegenen Punkte $F$ schneidet. Dieser Punkt wird durch den teilweise durchbrochenen Spiegel betrachtet und fält demnach bei der Anfangsstellung des Instrumentes mit dem Spiegelbild des Anfangspunktes der Kurve zusammen. Das Instrument wird nun so bewegt, daß immer der Schnittpunkt des Fadens mit $l$ mit den Kurvenpunkten, die im Spiegel erscheinen, zusammenfällt; ist der Endpunkt der Kurve erreicht, so entspricht der Indexpunkt der mittleren Ordinate. Das Instrument wird jetzt, nach Arretierung der Rolle, soweit gedreht, bis seine Fadenrichtung durch den ersten Auflagepunkt der Rolle in der Anfangsstellung hindurehgeht.

Sind $x, y$ die Koordinaten des optisch eingestellten Kurvenpunktes, $\xi, \eta$ des Berührungspunktes des Rädchens, so ist, wenn die Gerade $l$ dem Werte $x=0$ entspricht, immer $x=2 \xi$. Da di Richtung des Instrumentes (strenger des Fadens) den Weg der Rolle in jedem Augenblick tangiert, so ist

$$
\frac{y-\eta}{x-\xi}=\frac{d \eta}{d \xi}=\operatorname{tg} \tau,
$$

wo $\tau$ der Winkel der Tangente mit der Abszissenachse ist. Hieraus folgt

oder

$$
y d x=2 d(\eta \xi)
$$

$$
\frac{1}{2} \int_{x_{0}}^{x} y d x=\eta\left(\xi-\xi_{0}\right) \text {. }
$$

Da nun

$$
y_{m}=\frac{1}{x-x_{0}} \int^{0} y d x
$$

der Wert der gesuchten Mittelordinate ist, so ist mit Rücksicht auf $x=2 \xi, x_{0}=2 \xi_{0}, \eta=y_{m}$. Über die Verwendung des Instrumentes zur Aufzeichnung der mittleren Integralkurve oder im besonderen Falle zur Zeichnung von Hyperbeln s. Zeitschr. für Instrumentenk. 31, 69, 1911.

Von demselben Erfinder rührt ein Kompensationsplanimeter her, das im wesentlichen wiederum aus einer einfachen 
Stange mit Fahrstift besteht, die auf einer beweglichen Rolle rollt. Der scharfe Rollenrand findet in einer Rille des Stabes Halt, mit der der Stab a uf ihm aufgelegt wird. Damit die Rolle nicht umkippt, ist sie seitlich in einen auf der Unterlage rollenden Zylinder rerlängert. (Bei anderen Konstruktionen ist der Zylinder durch ein Rollenpaar oder durch eine Kugel ersetzt.) Ein weiterer Stützpunkt für den Stab ist neben dem Fahrstift am einen Ende angebracht. Das andere Ende des Stabes läuft in einen Index aus, an den eine Skala ungefähr senkrecht zum Stabe angelegt wird, die aber während der Umfahrung um eine Nadelspitze an einem ihrer Enden weggedreht werden kann, um die Bewregungen des Stabes nicht zu hindern. Er wird dann nach Beendigung der Umfahrung wieder gegen den Stabindex gelegt, um die Entfernung der Indexlagen (vor und nach der Umfahrung) zu messen, ähnlich wie beim Prytzschen Planimeter den Abstand der Marken. Die Rolle wird an einer Marke am Stabe eingestellt und kehrt nach der Lmfahrung genau oder wenigstens nahezu in diese Anfangslage zurück. Für letzteren Fall ist eine Korrektion anzubringen. Die Theorie (rgl. Zeitschr. f. Instrumentenk. 31, 173) schlieBt sich an die des rorigen Instrumentes an, indem der Stab sich in seiner Richtung doppelt so schnell wie die Rolle berregt. Die Genauigkeit ist annähernd gleich der des Coradischen Kompensationsplanimeters gefunden worden und übertrifft die des $\operatorname{Pr} 5$ tz schen Instrumentes bedeutend, während sie etwas unter derjenigen des freischwebenden Präzisionskugelplanimeters ron Coradi zurückbleibt.

\section{Abschnitt.}

\section{Apparate zur harmonischen Analyse.}

45. Allgemeines und Verwendung des Planimeters. Eine Funtition $y$ ron $x$ kann unter gewissen Einschränkungen in eine nach den cosinus und sinus der Vielfachen ron $x$ fortschreitende (Fourier sche) Reihe ent wickelt werden. Diese Funktion nehmen wir graphisch durch eine Kurre gegeben an, deren $A b-$ szissenintervall sich ron $x=0$ bis $x=u$ erstreckt. In diesem Intervall kann die Kurve vollständig willkürlich gezogen sein, so lange es für jedes $x$ nur einen einzigen endlichen Wert $y$ gibt, und hierin ist der Fall eingeschlossen, daB bei der Bewegung eines Punktes längs der Kurre die ihm entsprechenden Abszissen- 
werte stets zunehmen. Diese Bedingung wird für solche Kurven erfüllt sein, bei denen die $x$-Koordinate die Zeit darstellt, z. B. für alle registrierten Kurven, die durch Bewegung der Zeichenfläche mittels eines Uhrwerks entstanden sind. Unstetigkeiten der Kurve, d. h. ein plötzlicher Übergang von einer Ordinate $y_{1} \mathrm{zu}$ einer andern $y_{2}$ dürfen in endlicher Anzahl vorkommen. Ist das Zeichenpapier etwa um einen Zylinder geschlungen, so daß die Anfangs- und Endabszisse zusammenfallen, so wird sehr häufig die Anfangs- und Endordinate verschieden sein. Wenn z. B. die Lufttemperaturen für einen Zeitraum von 24 Stunden aufgezeichnet werden und die Stundenwerte die Abszissen, die Lufttemperaturen die Ordinaten geben, so werden die Mitternachtstemperaturen am Beginn und an Ende nicht übereinstimmen. Man kann dann die beiden Punkte der Kurve durch eine Mantellinie des Zylinders geradlinig verbunden denken und hat also in diesem Falle eine solche Stetigkeitsunterbrechung.

Wir setzen nun einen Winkelwert $\theta$ der Abszisse proportional $p x=\theta$ und $p u=2 \pi$, so daß $\theta=\frac{2 \pi x}{u}$ ist; dann können wir die Gleichung der Kurve in der folgenden Form schreiben, wo $a$ und $b$ konstante Koeffizienten sind:

$$
\begin{array}{r}
y=a_{0}+a_{1} \cos \theta+a_{2} \cos 2 \theta+a_{3} \cos 3 \theta+\cdots+a_{n} \cos n \theta \\
+b_{1} \sin \theta+b_{2} \sin 2 \theta+b_{3} \sin 3 \theta+\cdots+b_{n} \sin n \theta .
\end{array}
$$

Es ist dann nach Fourier

(2) $a_{n}=\frac{1}{\pi_{0}} \int_{0}^{2 \pi} y \cos n \theta d \theta, \quad b_{n}=\frac{1}{\pi_{0}} \int_{0}^{2 \pi} y \sin n \theta d \theta, \quad n=1,2,3, \ldots$ und $a_{0}=\frac{1}{2 \pi} \int_{0}^{2 \pi} y d \theta$ die mittlere Ordinate, die durch ein Planimeter ermittelt werden kann.

Streng genommen hat die Fouriersche Reihe eine unendlich große Gliederzahl, aber in den meisten praktischen Fällen sind die Glieder höherer Ordnung klein genug, um vernachlässigt zu werden. Beschränkt man sich auf $2 n$ Glieder, so kann man schreiben:

$$
y=a_{0}+\sum_{x=1}^{x=n} a_{x} \cos x \theta+\sum_{x=1}^{x=n} b_{x} \sin x \theta .
$$


Um die Koeffizienten zu finden, teilen wir das Abszissenintervall in $2 n$ gleiche Teile und messen die Ordinaten $y_{\lambda}=y_{1}, \ldots y_{2 n}$ in den Teilpunkten, wo $y_{\lambda}$ zu $\theta_{\lambda}=\frac{2 \pi x_{\lambda}}{u}$ gehört. Es müssen dann die zusammengebörigen Werte die Gleichungen befriedigen:

(4) $y_{\lambda}=a_{0}+\sum_{x=1}^{x=n} a_{x} \cos x \theta_{\lambda}+\sum_{x=1}^{x=n} b_{x} \sin \varkappa \theta_{\lambda}, \quad \lambda=1,2, \ldots 2 n$.

Diese $2 n$ linearen Gleichungen können zur Berechnung der $2 n$ Koeffizienten $a_{1}, \ldots a_{n}, b_{1}, \ldots b_{n}$ dienen

$$
n a_{x}=\sum_{i=1}^{2 n} y_{i} \cos x \theta_{\lambda}, \quad n b_{x}=\sum_{i=1}^{2 n} y_{\lambda} \sin * \theta_{\lambda}, \quad *=1 \ldots n \text {. }
$$

Für $x=0$ oder $*=n$ erhält man $2 n a_{0}{ }^{1}$ )

$\mathrm{Da}$ nun zwischen den Gleichungen (4) und (5) eine gewisse Reziprozität stattfindet, so läBt sich die Verwendung eines Instruments für die beiden Zwecke vorausseben, einmal die Koeffizienten einer gegebenen Kurre zu ermitteln, zweitens eine Kurve aus gegebenen Werten zu konstruieren.

Die hierzu erfundenen Instrumente heißen Analysatoren und sind also auch Instrumente zur Darstellung bestimmter Integrale wie die Planimeter.

In der Tat lassen sich diese letzteren nicht nur zur Ermittelung von $a_{0}$, sondern auch für die anderen Koeffizienten verwenden, wie S. Finsterwalder, P. Schreiber und Ad. Schmidt gezeigt haben. Um zunächst $a_{1}$ und $b_{1}$ zu erhalten, denkt man sich die Kurve um einen $Z y$ linder gelegt, so da $B$ die $x$-Achse den Grundkreis des Zylinders bildet, der den Umfang $u$ hat. Projiziert man die Kurve auf zwei zu einander senkrechte Ebenen, die sich in der Zylinderachse schneiden und von denen die eine durch die Endordinate gelegt ist, so erhält man die Koeffizienten $a_{1}$ und $b_{1}$ durch die Inhalte der von den Projektionen der Kurve, der $x$-Achse und den Endordinaten eingeschlossenen Flächen, wenn man noch

1) Setzt man $a_{x}=i_{x} \sin z_{x}, b_{x}=i_{x} \cos \gamma_{x}$, so kaun man $a_{x} \cos x \theta+$ $b_{x} \sin x \theta=i_{x} \sin \left(\gamma_{x}+x_{x} \theta\right)$ schreiben. Es ist in vielen Fällen wünschenswert, die Amplituden $i_{x}$ und die Phasenwinkel $\gamma_{x}$ statt $a_{x}$ und $b_{x}$ zu erbalten. Um dies zu erleichtern, hat Strachey in London einen besonderen Rechenschieber konstruiert (vgl. W. Dyck Katalog mathematischer usw. Instrumente, München, 1892, S. 144). 
mit $\frac{u}{2}$ dividiert. Die Ebene, die durch den Anfangspunkt der Kurve geht, dient zur Berechnung von $b$. Hier wird $\sin \theta$ gleichzeitig mit $\theta$ null, während auf der dazu senkrechten Ebene (für die Berechnung von $a$ ) sich das Linienelement des Anfangspunkts unverkürzt abbildet und $\cos \theta=1$ für $\theta=0$ ist. Wenn die Quadranten der Winkel beachtet werden, so erhält man die Flächenteile auf beiden Seiten der Projektionsebene (vorn und hinten) mit verschiedenen Vorzeichen, wie es sein muB.

Die anderen Koeffizienten lassen sich in entsprechender Weise bestimmen. Man setrt $n \theta=n \frac{2 \pi x}{u}=\frac{2 \pi x}{u^{\prime}}$ oder $u^{\prime}=\frac{u}{n}$, nimmt also einen Zylinder rom Umfang $\frac{u}{n}$. Da $x$ von 0 bis $u=n u^{\prime}$ variiert, so umschlingt die Kurve den Zylinder $n$-mal. Man kann ebenso gut $n \frac{2 \pi x}{u}=\frac{2 \pi x^{\prime}}{u}$ setzen, also $x^{\prime}=n x, \mathrm{~d}$. b. die Abszissen $n$-mal vergröBert auf einen Zylinder vom Umfange $u$ auftragen (während die Ordinaten unverändert bleiben). Da aber in dem Integral (2) $d x^{\prime}$ statt $d x$ eingeführt wird, so muB man mit $\frac{2}{n u}$ statt mit $\frac{2}{u}$ multiplizieren.

Bei der wirklichen Verzeichnung der auszumessenden Kurven denkt man sich den Zylinderumfang in eine solche Anzahl von gleichen Teilen geteilt, daß die Ordnungszahlen der Reibenkoeffizienten als Faktoren in dieser Anzahl enthalten sind (z. B. 60 bei Beschränkung auf 6 Glieder, 24 bei 4 Gliedern) und zieht die den betreffenden Mantellinien des Zylinders entsprechenden $\mathrm{G}_{\theta}-$ raden in der Projektion. Trägt man dann die Ordinaten auf, so erhält man durch die geradlinige Verbindung ihrer Endpunkte die gesuchten Kurven mit genügender Genauigkeit und kann sie nun mit dem Planimeter ausmessen.

46. Analysatoren von Yule. Eine einfache Form eines Analysators, die man sich selbst herstellen kann, ist von Yule beschrieben worden. An der Seite eines größeren quadratischen Kartonblattes ist ein Lineal befestigt (etwa aufgeleimt), an dem sich ein kleines viereckiges Kartonblatt verschieben läßt, das am Lineal anliegen muB und einen Zeiger parallel der $x$-Achse trägt. Die untere Kante des großen Blattes wird mit der Richtung der $x$-Achse zusammengelegt und zwar so, da $B$ der Zeiger, wenn er unten einsteht, auf deu Anfangspunkt der Kurve weist. Das Ab- 
szisseninterrall teilt man z. B. in 60 Teile, je $6^{0}$ entsprechend und errichtet die Mittelordinaten, deren zugehörige Kurrenpunkte man nach und nach mit dem Zeiger durch Terschiebung des kleinen Blattes in der $y$-Richtung einstellt, indem man den groBen Karton gleichzeitig längs der $x$-Achse rerschiebt. Auf dem kleinen Karton bringt man z. B. ëine sinus-Skala, ebenfalls in der Richtung der $x$-Achse, an und stellt den Fahrstift eines Amslerschen Polarplanimeters jedesmal auf den der betreffenden Ordinate entsprechenden Teilstrich, also wenn man mit $0^{\circ}$ anfängt, schiebt man ihn immer um je $6^{0}$ weiter. Der Pol des Planimeters ist fest auf dem groBen Karton. Infolge dessen umfährt der Stift des Planimeters folgende Flächen:

$y_{1}\left(\sin 6^{\circ}-\sin 0^{\circ}\right), y_{z}\left(\sin 12^{0}-\sin 6^{0}\right), y_{3}\left(\sin 18^{0}-\sin 12^{0}\right), \cdots$,

$$
y_{n}\left(\sin 360^{\circ}-\sin 354^{\circ}\right) \text {. }
$$

Wir erhalten also genähert

$$
\int y d(\sin \theta)=\int y \cos \theta d \theta=a
$$

und durch Einsetzen anderer Skalen und andere Wahl des $\mathrm{Ab}$. szissenintervalls andere Koeffizienten.

Nicht ganz so einfach ist ein anderes Instrument von Yule. Es besteht aus einem Lineal, das auf zwei Walzen an seinen

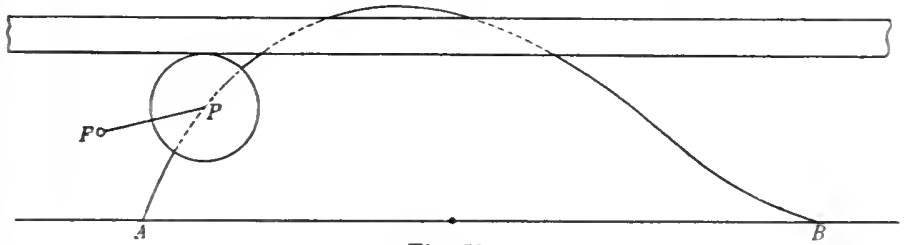

Fig. 70.

Enden rollt und sich dadurch parallel mit sich selbst verschieben kann. Längs der einen Kante trägt es eine Zahnstange ron $30 \mathrm{~cm}$ Länge (mit acht Zähnen auf einen Zentimeter), die durch ein Gegengewicht an der entgegengesetzten Kante im Gleichgewicht gehalten wird, so da $B$ das Lineal horizontal liegt. Eine am Rande gezahnte Scheibe wird so angelegt, dab sie in die Zahnstange eingreift. Bei der Bewegung der Scheibe längs der Kurve muB das Lineal angeschoben werden, damit die Scheibe in die Zahnstange eingreift. AuBer einer groBen Scheibe mit 240 Zähnen, 
die sich bei der Bewegung längs der Zahnstange also gerade einmal herumdreht, werden nach einander noch kleinere Scheiben mit 120, 80 usw. Zähnen verwendet. Die große Scheibe hat drei Löcher in der Richtung ejnes Durchmessers, von denen das mittelste mit einem Glasfenster und einem Punkt darauf zur Einstellung auf die Punkte der Kurve versehen ist, die beiden anderen dienen zur Orientierung der Scheibe. Ein viertes kegelförmiges Loch auf einem zum vorigen senkrechten Durchmesser hat den Zweck, den Fahrstift eines Polarplanimeters aufzunehmen. Seine Entfernung vom Mittelpunkt der Scheibe (Fahrpunkt) beträgt $\frac{10}{\pi} \mathrm{cm}$. Bei den kleineren Rädern, die auf einer Grundplatte angebracht sind, befindet sich das Loch für den Planimeterstift auf einem über das Rad hervorragenden Arm, der sich mit dem Rade frei über der Zahnstange des Lineals dreht.

Das Abszissenintervall $2 u$ macht man der Länge der Zahnstange gleich, der Scheibenumfang ist dann ein aliquoter Teil desselben $\frac{2 u}{n}$. Das Loch $F$ für den Fahrstift des Planimeters beschreibt bei der Drehung der Scheibe einen Kreis mit dem Radius $P F=r$ um den Mittelpunkt der Scheibe $P$ (Fig. 70). Im Anfangspunkte sei $P F$ nach links in die Richtung der Abszissenachse gestellt. Da sich die Scheibe $2 n \pi$-mal herum dreht, wenn sie die Kurve entlang von $A$ bis $B$ geführt wird, so entspricht einer Strecke $x$ ein Drehungswinkel $n \pi \frac{x}{u}=n \theta$, wenn $\frac{\pi}{u} x=\theta$ gesetzt wird. Die Koordinaten $\xi, \eta$ von $F$ sind dann folgende, wenn $x, y$ diejenigen von $P$ sind:

$$
\xi=x-r \cos n \theta \quad \eta=y+r \sin n \theta .
$$

Daher ist die vom Planimeterstift umfahrene Fläche

$\int \eta d \xi=\int y d x+n r \int y \sin n \theta d \theta+r \int \sin n \theta d x+n r^{2} \int \sin ^{2} n \theta d \theta$.

Die beiden letzten Integrale verschwinden, wenn sie sich über eine geschlossene Kurve erstrecken, also die obere und untere Grenze für $x$ bzw. $\theta$ dieselbe ist. Beim zweiten Integral ist der Wert derselbe, ob man es über die Kurve von $A$ bis $B$ nimmt, oder ob man die Fläche ganz umfährt, also auch von $B$ nach $A$ in der Abszissenachse zurückgeht, da diese letztere Strecke keinen Beitrag zu dem Integrale liefert, sondern $y=0$ bleibt. Setzen wir 


$$
\int y d x=f \text { und } \int \eta d \xi=B_{n}
$$

so ist, wenn noch $r=\frac{10}{\pi}$ eingesetzt wird (vgl. (2) S. 132):

$$
B_{n}=f+10 n \cdot b_{n} \text {. }
$$

Wird die Scheibe im Anfangspunkte $A$ so gestellt, daB $P F$ mit der positiven Richtung der $y$-Achse zusammenfällt, so ist

also

$$
\xi=x+r \sin n \theta \quad \eta=y+r \cos n \theta,
$$

$$
\begin{aligned}
A_{n}=\int \eta d \xi=\int y d x & +n r \int y \cos n \theta d \theta+r \int \cos n \theta d x \\
& +n r^{2} \int \cos ^{2} n \theta d \theta
\end{aligned}
$$

und entsprechend wie vorher

$$
A_{n}=f+10 n \cdot a_{n}, \quad B_{n}=f+10 n \cdot b_{n} .
$$

Jede Scheibe $n$ liefert also ein Koeffizientenpaar $a_{n}, b_{n}$. ( $f$ wird mit einem Planimeter gemessen.)

47. Apparat von Le Conte. Bei dem Apparat von Le Conte sind auf einer Grundplatte ein Schienenpaar ( $D E, F G$ Fig. 71),

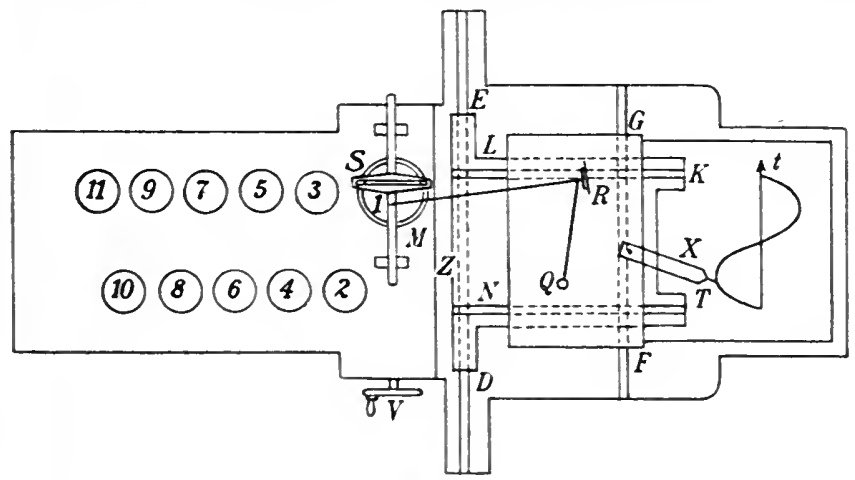

Fig. 71.

auf dem ein Wagen läuft, und elf Zahnräder angeordnet, die durch eine Kurbel $V$ in Umdrehung gesetzt werden und zwar macht jedes Rad bei einer Kurbeldrehung seiner Numerierung entsprechend $1,2,3 \ldots$ usf. Umdrehungen. Der Wagen wird durch dieselbe Handkurbel bei einer Umdrehung des Rades 1 um die Strecke, 
die einer Periode entspricht, vorwärts bewegt. Ein zweites Schienenpaar $(L K, N T)$, das auf dem Wagen rechtwinklig zum ersten angebracht ist, trägt einen zweiten Wagen, an dem der Fahrstift $X$ angebracht ist. Durch die Bewegung des ersten Wagens wird er parallel der $x$-(in der Fig. $t_{-}$) Achse geführt, während seine Einstellung auf die Ordinatenendpunkte eine Verschiebung des zweiten Wagens verursacht.

Das Folgende wird durch die Überlegung verständlicher, daB die Projektion eines gleichförmig im Kreise sich bewegenden Punktes auf einen Durehmesser des Kreises eine harmonische Bewegung ausführt.

Das Zahnrad 1 (und ebenso jedes der übrigen) trägt einen exzentrischen Stift $S$, der in einen Schlitz eingreift. Der Schlitz befindet sich in dem kürzeren Arm eines kreuzförmigen Schlittens, dessen längerer, der $x$-Achse paralleler Arm durch die Bewegung von $S$ sich längs eines Durchmessers des Rades hin- und herschiebt. Wenn sich $S$ im Kreise um den Mittelpunkt des Rades dreht, so vollführt seine Projektion auf den der $x$-Achse parallelen Durchmesser eine harmonische Bewegung $(r \cos \theta$ bzw. $r \sin \theta$, je nachdem die Anfangsstellung des Stifts in dem der $y$-oder $x$-Achse parallelen Durchmesser sich befand). Setzt man auf den Schlitten $M$ den Fahrstift eines Polarplanimeters, so beschreibt dieser daher dieselbe Bewegung. Der Pol $Q$ und die MeBrolle $R$ des Planimeters, werden auf den zweiten Wagen aufgesetzt. Wenn der Fahrstift des Analysators und mit ihm der zweite Wagen in der $y$-Achsenrichtung verschoben werden, so wird dabei dasselbe erreicht, als wenn bei ruhender Planimeterebene der Fahrstift des Planimeters in der entgegengesetzten Richtung der $y$-Achse geführt wird. Die Theorie ist genau dieselbe, wie beim vorigen Planimeter.

Um die Koeffizienten $a_{2} \ldots a_{11}, b_{2} \ldots b_{11}$ zu erhalten, wird der Planimeterfahrstift auf die (in der Figur fehlenden) Schlitten der anderen Räder gesetzt.

48. Analysator von W. Thomson. Älter als die genannten Instrumente ist der 1876 von William Thomson (Lord Kelvin) konstruierte Analysator ${ }^{1}$ ), der an das von seinem Bruder James Thomson erfundene Planimeter anknüpft.

1) Proceedings of the Royal Society of London, XXIV, 1876. 
Wir denken uns (ähnlich wie bei A. Schmidts Planimeter, S. 116, vgl. auch S. 133) die auszumessende Kurve $y=f(x)$ um einen horizontalen „Kurvenzylinder" gelegt, so da $B$ die $x$-Achse etwa mit dem Grundkreise zusammenfällt und die $y$-Achse der Zylinderachse parallel ist. Durch Zahnradübertragung wird der Achse $A$ einer Scheibe $D$ eine mit der Drehung $d x$ des Zylinders proportionale Drehung $p d x$ erteilt (Fig. 12 ). Diese Scheibe ist gegen die Horizontalebene um $45^{\circ}$ geneigt und auf ihr rollt eine frei bewegliche Kugel $B$, die durch einen zweiten horizontalen Zylinder $C$, den wir zum Unterschied den „Registrierzylinder" nennen, und der die Scheibe nicht berührt, längs des horizontalen Durchmessers der Scheibe
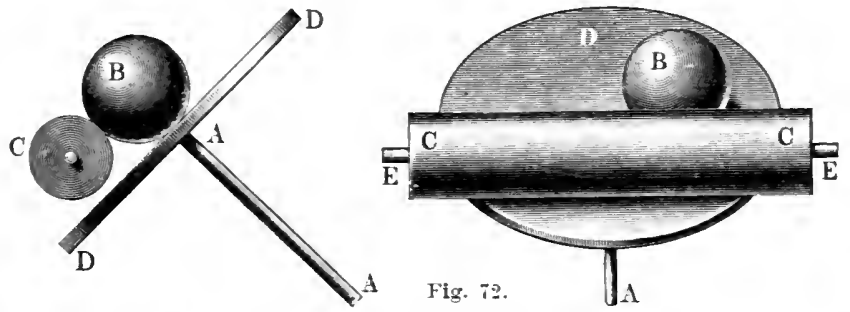

zu rollen gezwungen wird. Die Kugel berührt demnach sowohl die Scheibe als den Zylinder und überträgt die Drehberegungen der Scheibe durch Reibung auf den Zylinder, so daB bei einem Abstande $y$ des Berührungspunktes der Kugel rom Mittelpunkt der Scheibe $p y d x$ die Drehung des Zylinders ist. Beide Zylinderachsen sind einander parallel und eine zu ihnen parallele Stange trägt an einem Eude den Fahrstift, der auf der jedesinal oben befindlichen Mantellinie des Kurvenzylinders auf den Kurvenpunkt eingestellt wird. Am anderen Ende der Stange befindet sich eine Gabel, welche die Kugel ron oben faBt und längs des Registrierzylinders und des horizontalen Scheibendurchmessers hin- und herschiebt (vgl. Fig. 29). Wenn der Fahrstift auf der $x$-Achse steht, berührt die Kugel die Scheibe im Mittelpunkte, daher ist ihr Abstand ron diesem immer gleich der Ordinate $y$ der Kurre. In geeigneter Weise wird die Drehung des Registrierzylinders gemessen, die für ein Kurvenstück zwischen den Abszissen $x_{1}$ und $x_{2}$ den Integralwert $\int_{x_{1}}^{x_{2}} y d x$ bis auf einen konstanten von den Abmessungen des Instrumentes abhängigen Faktor gibt. 
Diesen Planimeterapparat hat W. Thoms on in folgender Weise zu einem Analysator abgeändert. Die Achse des Kurvenzylinders trägt einen Exzenter, also eine Vorrichtnng, welche die Drehbewegung in eine geradlinige verwandelt. Eine Beschreibung ist nicht angegeben, doch kann man sich die Einrichtung wie beim Le Conteschen Analysator vorstellen, wo die Drehung der Scheibe in die geradlinige Schlittenbewegung verwandelt wird (vgl. S. 138). Durch Einschaltung verschiedener Zahnräder kann man erlangen, daB einer Umdrehung des Zylinders $x$ hin- und hergehende Bewegungen einer Stange entsprechen. Jeder Punkt der Stange macht also eine geradlinige Bewegung, deren Größe proportional $\sin x \theta$ bzw. $\cos x \theta$ ist, je nach der Anfangsstellung. Die Stange ist gezahnt, greift in den gezahnten Rand der Scheibe ein und erteilt ihr eine Drehung, bei der $d x$ proportional $\cos x \theta \cdot x d \theta$ bzw. $\sin x \theta \cdot x d \theta$ ist. Mithin sind die Ablesungen am Registrierzylinder bis auf Faktoren, die von den Dimensionen des Instruments abhängen,

$$
\int y \cos x \theta d \theta \text { und } \int y \sin x \theta d \theta,
$$

also die gesuchten Koeffizienten $a_{x}$ und $b_{x}$.

Sollen mehrere Koeffizienten gleichzeitig bestimmt werden, so müssen mit einem Kurvenzylinder und einem Führungsstift mehrere Integratoren verbunden werden, welchen ebenso viele Registrierzylinder zugehören. Der Apparat hat Fehler gezeigt, die von dem Gleiten der Kugel bei raschem Arbeiten herzurühren scheinen, auch berühren die Kugeln in kleinen Flächenstücken statt in Punkten.

49. Analysator von Henrici-Coradi. Vollkommener, aber auch komplizierter ist der Analysator von Henrici, der von Coradi konstruiert wurde. Er beruht auf dem sog. Henricischen Prinzipe, das die Koeffizienten $a$ und $b$ durch partielle Integration umwandelt, es ist nämlich:

$$
\begin{gathered}
a=\int_{0}^{2 \pi} y \cos \theta d \theta=[y \sin \theta]_{0}^{2 \pi}-\int_{0}^{2 \pi} \sin \theta d y \\
b=\int_{0}^{2 \pi} y \sin \theta d \theta=[-y \cos \theta]_{0}^{2 \pi}+\int_{0}^{2 \pi} \cos \theta d y .
\end{gathered}
$$


Die Integrale erstreeken sich über die ganze Periode, infolge dessen fällt das erste Glied auf der rechten Seite fort ${ }^{1}$ ) und das rechts stehende Integral hat eine wesentlich andere Form, als das links stehende.

Das Instrument ron Henrici hatdurchSharp und namentlich durch $\mathrm{Co}_{0}$ radi wesentliche Verbesserungen erfahren und ist schlieBlich zu folgender Gestalt gelangt:

Ein festes Gestell, dessen Grundriß ein langes Rechteck ist, ruht mit drei Rollen auf der Zeichenebene. Eine ron ihnen, vorn in der Mitte gelegen, dient nur als Stütze, die beiden anderen sind an den Enden einer langen Achse angebracht. Wir bezeichnen sie als Haupt-

1) Dies ist nur dann nicht der Fall, wenn die Ordinate einen Sprung macht, also wenn die Kurse eine Unstetigkeitsstelle besitzt. Seien in diesem falle die zu einem Abszissenwerte $x$ gehörenden Kursenpunkte $P_{1}$ and $P_{1}^{\prime}$ mit den Ordina. ten $y_{1}$ und $y_{1}^{\prime}$ und $\theta^{\prime}$ der zugehörige Wert ron $\theta$, so tritt bei $a$ hinzu: $\left(y_{1}-y_{1}^{\prime}\right) \sin \theta^{\prime}$, bei $b$ hinzu: $\left(y_{1}-y_{1}\right) \cos \theta^{\prime}$. Für $\theta^{\prime}=0$ oder $2 \pi$ wird der erste Ausdruck null.

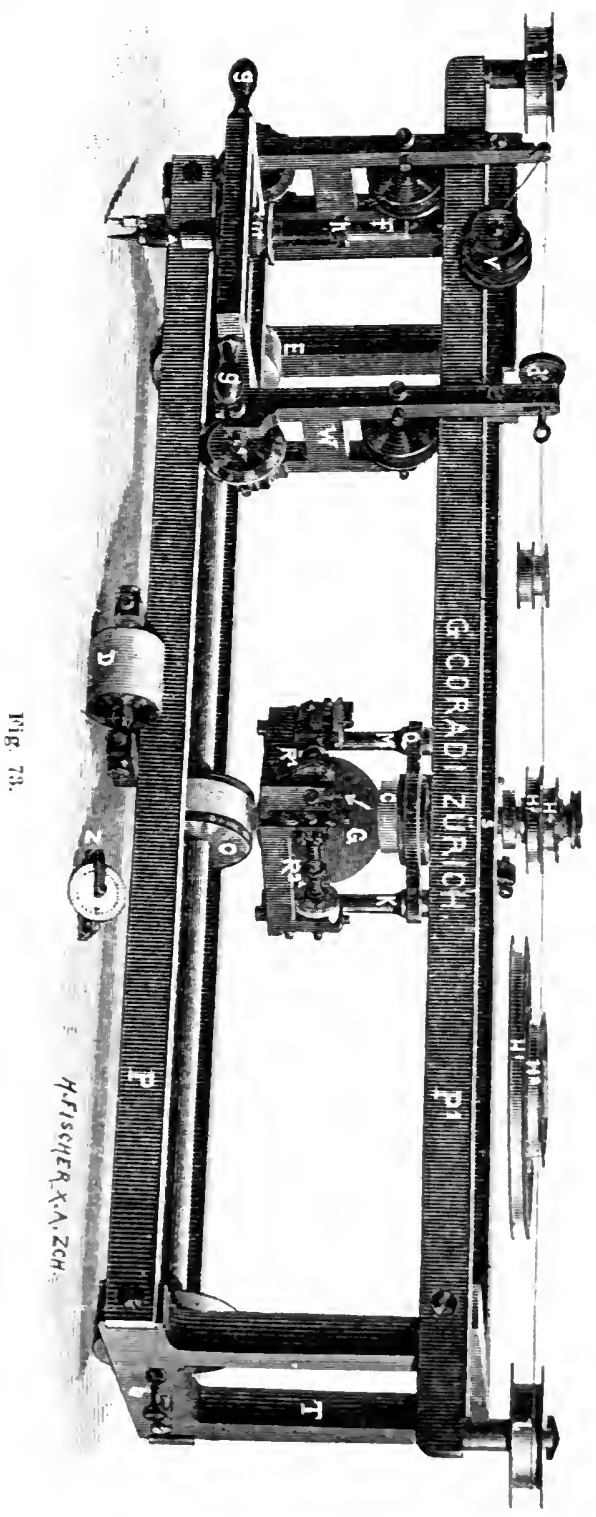


achse und wählen ihre Richtung als $x$-Achse, so daB das Instru ment in der Richtung der Ordinaten $y$ rollt. Bewegt es sich un ein Stück $d y$, so drehen sich die Rollen um $d \varphi=p d y$, wo $p$ eil Proportionalitätsfaktor ist. Auf der Hauptachse sitzt eine Anzah unter einander gleicher walzenförmiger Zylinder $O$ (in der Figur is nur einer gezeichnet), über deren jedem ein Integrator mit vertikale Achse angebracht ist, der je einen der Koeffizienten $a_{x}, b_{*}$ liefert Die geometrischen Mittellinien dieser Vertikalachsen schneides sämtlich die Hauptachse und zwischen diesen Vertikalachsen ode Zapfen, wie, wir sie nennen wollen und den walzenförmigel horizontal liegenden Zylindern liegt jedesmal eine Glaskugel $G$ Die Zapfen $C$ sind in dem hinteren erhöhten Rahmen $s$ befestigt

Bevor wir die Integratoren eingehender beschreiben, betrachteI wir die Vorrichtung, welche eine Drehung der Zapfen bewirkt Entlang der Vorderseite $P$ des Rahmengestells läuft ein Wagen $W$ an dem der Fabrstift $F$ befestigt ist, der einen Weg gleich de: Basis $u$ der gezeichneten Kurven durchlaufen kann, die also den Di mensionen des Instruments angepaßt werden muß. An dem Wager ist oben ein Silberdraht befestigt, der auf der Vorderseite de Rahmens entlang gespannt ist und, über zwei horizontale Füh rungsrollen $l$ an beiden Enden des Apparats geführt, sich um klein Scheiben $H$ schlingt, die ebenfalls horizontal oben auf deu Zapfer befestigt sind. Durch passende Wahl der Durchmesser diese Scheiben wird es erreicht, daß sich der Zapfen $x$-mal um seine Achs dreht, wenn der Stift die ganze Basis durchläuft. Die Durchmesser dieser Schnurscheiben verhalten sich wie $1: \frac{1}{3}: \frac{1}{5}$ usf. Wenn sich also der Fahrstift um $x$ bewegt, so drehen sich die Scheiben ur $\vartheta, 3 \vartheta, 5 \vartheta$ usf., wenn $\vartheta=\frac{2 \pi x}{u}$ ist.

Mit jedem Zapfen ist unten ein horizontaler viereckiger Rahmer verbunden (Fig. 73). Zwei Seiten desselben werden von den Achser zweier Integrierrollen $R_{1} R_{2}$ (Fig. 74) gebildet, welche die er wähnte, im Innern des Rahmens liegende Glaskugel $G$ berühren die von der dem Schnitt $I$, der Rollenachsen gegenüberliegenden Eck aus durch eine Führungsrolle $r$ an die Integrierrollen angedrück 1 wird. $\left.{ }^{1}\right)$ Bewegt sich der Fahrstift in der Richtung der $x$-Achse bis zum Punkte $x$, so dreht sicl die Zapfenscheibe mit dem Radius

1) Die Anordnung der Kugel mit zwei MeBrollen rübrt ron Mas Küntzel, Markscheider in Charlottenhof bei Königshütte $0 / \mathrm{S}$ her. 
$\frac{1}{*}$ um $\% \vartheta$ (in der Figur mit $k \omega t$ bezeichnet, mit $t$ die $x$-Achse) und die Integrierrolle $R_{1}$ bildet, wenn sie vorher der $x$ Achse parallel war, jetzt den Winkel $\% \vartheta$ mit ibr; die Ebene der Integrierrolle $R_{9}$ wird den Winkel $90^{\circ}+x \vartheta$ mit der $x$-Achse einschlieBen. Wird der Apparat um $d y$ fortgerollt, so wird die Drehung der Zylinderwalze $p d y$ sich der Kugel mitteilen, die demnach $c d y$ sei, und die Integrierrollen werden, da die Kugel sich senkrecht zur $x$ Achse um einen dièser parallelen Durchmesser dreht, sich proportional $c d y \cos \% \vartheta$ bzw. $c d y \sin x \vartheta$ drehen. Bei

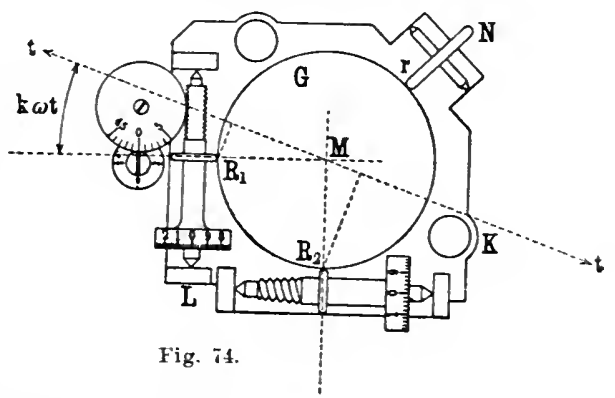
einer Bewegung des Fahrstifts längs der Kurve werden daher ron den Registrierrollen die Koeffizienten

$$
a_{x}=c \int^{d} d y \cos x \vartheta \text { und } b_{x}=c \int^{2} d y \sin x \vartheta
$$

geliefert. Um nicht zu viele Integratoren nötig zu machen, sind an jedem Zapfen mehrere Schnurscheiben von rerschiedenem Durchmesser vorgesehen, über welche der Silberdraht geführt werden kann. $\left.{ }^{1}\right)$

50. Analysator von Sharp. Der Analysator von Sharp hat wiederum ein auf drei Rollen laufendes Gestell, das aber im Grundrisse die Gestalt eines doppelten $U$ hat (Fig. 75). Die Enden der drei Arme liegen in gerader Linie, die Richtung der Arme wählen wir als Richtung der Abszissen (in der Figur $t$ ). An den Enden der beiden äuBeren Arme $F$ befinden sich Rollen $W_{1}, W_{2}, W_{3}$ ron verschiedenem Durchmesser im Verhältnis $1: \frac{1}{2}: \frac{1}{3}$ usf., dic auf der Papierebene oder

1) Wenn die Achsen der Zapfen nicht über den Kugelmittelpunkten $M$ liegen, so entstehen Fehler, die sich aber durch Befahren der Kurve in entgegengesetzter Richtung eliminieren lassen. Bezüglich aller Einzelheiten der Konstruktion, der Behandlung des Instruments und der Fehlertheorie muB auf die eingehende Theorie des harmonischen Analysators von Dr. L. Grabowski (Sitzungsberichte der math. naturw. Klasse der K. Akademio der Wissenschaften in Wien, Bd. 110, Abt. II a, S. 717-889, Wien 1901) verwiesen werden. Das dort mitgeteilte Beispiel zeigt, wie die Resultate durch Anbringung von Korrektionen wesentlich verbessert werden. 
auf Leisten laufen. An dem rückwärts verlängerten Mittelarm sitzt noch eine Stützrolle $W$. An seinem Ende $M$ trägt dieser Mittelarm eine Scheibe $D_{1}$, die sich durch Zahnradübertragung bei der Bewegung des Apparats um das ganze Abszissenintervall $u$ einmal herumdreht (bei Anwendung der Rollen mit dem gröBten Durchmesser, bzw. xmal bei den Rollen vom Durchmesser $\left.\frac{1}{x}\right)$. Bei einer Bowegung des Instruments um $x$ dreht sie sich um $\vartheta=\frac{2 \pi}{u} x$ (bei Verwendung der Rollen mit dem Durchmesser $\frac{1}{x}$ um $x \vartheta$ ).

Auf dem langen Rahmen, an dem die drei Arme sitzen, bewegt sich auf einer Schiene $S$ ein Wagen in der Richtung der $y$ -

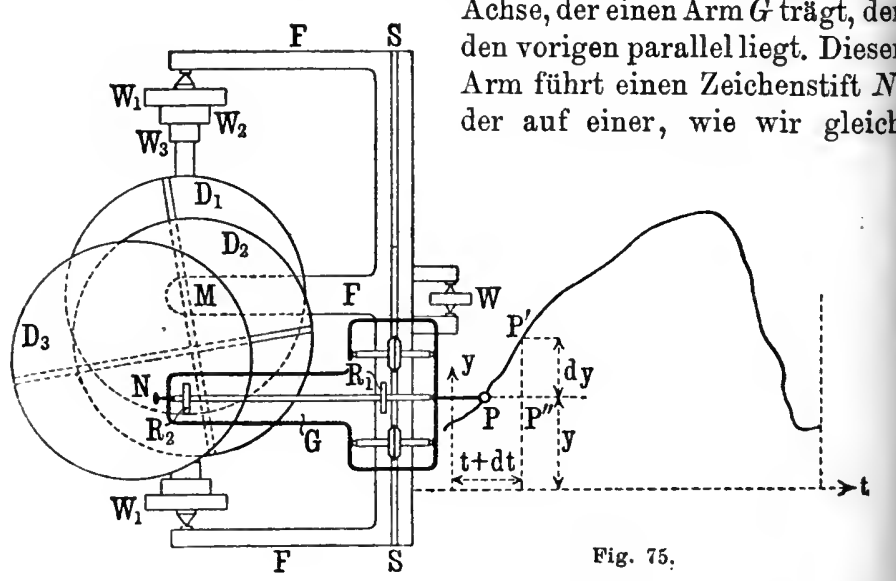

sehen werden, mit der bereits genannten Scheibe $D_{1}$ sich drehenden Scheibe $D_{3}$ zeichnet und in seiner Rückverlängerung den Fahrstift $P$, der entlang der gegebenen Kurve geführt wird. Weil nämlich die Scheibe $D_{1}$ fest sitzt, und der Wagen sich in der $y$-Richtung bewegt, so mußte eine in gewissen Greuzen mit dem Schreibstift $N$ mitgehende Scheibe angebracht werden. Deshalb ist auf $D_{1}$ längs eines Durchmessers eine Führungsleiste angebracht, die in eine entsprechende Nut längs des Durchmessers einer zweiten Scheibe $D_{2}$ eingreift. In derselben Weise steht mit $D_{2}$ eine dritte Scheibe $D_{3}$ in Verbindung. $D_{3}$ trägt auf der Unterseite eine Führungsleiste, die in einer auf $D_{2}$ befindlichen, zur anderen senk- 
recht verlaufenden Nut sich verschiebt. Da nun neben dem Zeichenstift eine Friktionsrolle $R_{g}$ an dem beweglichen Arm angebracht ist, welche die mit Papier überzogene Scheibe $D_{3}$ im Berührungspunkte festhält, so dreht sich $D_{3}$ um diesen Punkt um denselben Winkel $\vartheta$ bzw. $x \vartheta$, wie die Scheibe $D_{1}$, wenn das Instrument um eine Strecke $x$ (Richtung $t$ in der Figur) fortrollt. Wenn dagegen der Wagen um $d y$ bewegt wird, so rollt das Friktionsrädchen, und mit ihm bewegt sich der Zeichenstift um diesen Betrag. Hat man in der Anfangslage der Scheibe $D_{3}$ Parallele zur $x$ - und $y$-Achse gezogen, so hat sich dieses Koordinatenkreuz um $\vartheta$ gedreht, daher sind die Komponenten ron $d y$, abgesehen rom Vorzeichen $\cos \vartheta d y$ und sin $\vartheta d y$ und bei Durchfahrung der ganzen Kurve erhält man $\int \cos \vartheta d y$ and $\int \sin \vartheta d y$ bzw. $a_{x}=\int \cos x \vartheta d y$ und $b_{x}=\int \sin x \vartheta d y$ bei Einschaltung der Rollen mit dem Durchmesser $\frac{1}{x}$. Die Verbindungsgerade des Anfangs- und Endpunkts der auf der Scheibe dargestellten Kurve liefert dann die Periode $\gamma_{*}$ und die Amplitude $i_{x}$ durch

$$
\frac{b_{x}}{a_{x}}=\operatorname{tg} \gamma_{x} \text { und } i_{x}=\sqrt{a_{x}{ }^{2}+b_{x}{ }^{2}}
$$

und damit die Koeffizienten bei der Darstellung der Kurre in der Form: $i=\int i_{x} \sin \left(x \vartheta+\gamma_{x}\right)$, wo $b_{x}=i_{x} \sin \gamma_{\varkappa}, a_{x}=i_{x} \cos \gamma_{x}$ ist.

51. Präzisionsinstrument von Sommerfeld und Wiechert. Ein Präzisionsinstrument ist der harmonische Analysator von A. Sommerfeld und E. Wiechert, konstruiert ron Volkmann und Gross in Königsberg i/P., 1890. ${ }^{1}$ )

Die Maschine besteht in den Hauptteilen aus einem horizontal liegenden Zylinder $a b c d$ (Fig. 76), der erstens um seine Umdrehungsachse und zweitens um eine Vertikalachse $C$, die wir Konstruktionsachse nennen, drehbar ist. Parallel der Konstruktionsachse ist die sogenannte Integrationsachse $J$ angebracht, um die sich eine horizontale kreisförmige

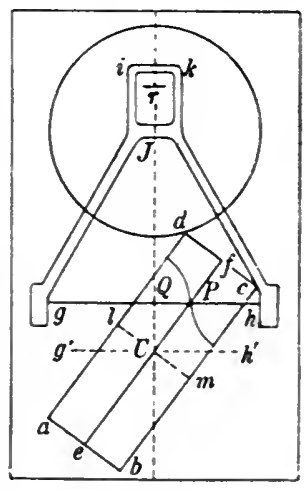

Fig. 76.

1) Sitzungsberichte der physik.-ökonomischen Gesellscb. zu Königsberg, Bd. 32, S. 28, 1891. 
Scheibe dreht. Wenn sich der Zylinder um seine Rotationsacbse um einen Winkel $\vartheta$ dreht, so dreht sich die Scheibe um denselben Winkel, und gleichzeitig dreht sich durch eine noch zu erwähnende Vorrichtung der Zylinder um die Konstruktionsachse, also im azimutalen Sinne, um den Winkel $k \vartheta$. Die gegebene Kurve ist auf dem mit Papier überspannten Zylinder aufgezeichnet. ${ }^{1}$ ) Die bei seiner Drehung jeweilig oberste Mantellinie wird durch einen längs derselben gespannten Faden ef markiert. Derjenige Punkt $P$ der Kurve, der unter diesen Faden kommt, wird mittels eines andern ebenfalls horizontal liegenden Fadens $g h$ eingestellt. Dieser Einstellfaden läBt sich durch einen Schieber (Wagen $i k$ ) parallel verstellen. Am Ende des Schiebers befindet sich eine Integrierrolle $(r)$, deren Achse der Verschiebungsrichtung parallel ist. Die Rolle liegt auf der Scheibe auf und verschiebt sich längs eines Durchmessers derselben. Bei der Einstellung des beweglichen Fadens $g^{\prime} h^{\prime}$ auf die $x$-Achse der Kurve, die einen dem Grundkreis des Zylinders parallelen Kreis bildet, berührt die Rolle die Scheibe im Mittelpunkt. Der Abstand des Berührungspunkts der Rolle vom Mittelpunkt der Scheibe mißt also $y \cos k \vartheta$ oder $y \sin k \vartheta$, je nachdem die Zylinderachse den Winkel $J C P=k \vartheta$ oder $\frac{\pi}{2}-k \vartheta$ mit der Schieberrichtung einschließt, wobei $\vartheta$ der der Abszisse $x$ entsprechende Winkel, $\vartheta=\frac{2 \pi x}{u}$, ist, und $u$ den Umfang des Zylinders bezeichnet. Bei einer Winkeländerung $d \vartheta$, welche sowohl die Umdrehungsbewegung des Zylinders, wie die der Scheibe betrifft, wird daher die Rolle den Weg $y \cos k \vartheta \cdot d \vartheta$ oder $y \sin k \vartheta \cdot d \vartheta$ abwickeln; bei einer vollen Umdrehung des Zylinders um seine Achse erbält man als Ablesungen der Integrationsrolle

$$
a_{x}=\int_{0}^{2 \pi} y \cos k \vartheta d \vartheta \text { bzw. } b_{x}=\int_{0}^{2 \pi} y \sin k \vartheta d \vartheta .
$$

1) Die Kurve kann von dem Apparate selbst anfgezeichnet werden; zu diesem Zwecke wird die Zylinderachse der Schieberrichtung parallel gestellt. Am Schieber wird ein Zeichenstift angebracht, dem man mit Hilfe einer Skala eine der $y$-Koordinate entsprechende Stellung geben kann. Gleichzeitig wird dem Zylinder eine wachsenden Abszissen $x$ entsprechende Drehung erteilt und der Zeichenstift heruntergedrückt. Dieser zeichnet durch eine ihm erteilte Rotationsbewegung einen kleinen Kreis, dessen Mittelpunkt unabhängig von einer etwa vorhandenen Exzentrizität des Stifts den richtigen Punkt bezeichnet. 
Die Drehung des Zylinders im azimutalen Sinne beruht auf folgender Einrichtung (Fig. 77): Die vertikale Achse, um die sich der Zylinder dreht und die mit der Konstruktionsachse $C$ zusammenfällt, ist zugleich Achse eines in der Nähe ihres unteren Endes angebrachten borizontalen Rades $r_{1}$, dessen Radius wir ebenso bezeichnen, wie auch im folgenden entsprechend bei anderen Rädern. Sie ruht mit ihrem unteren Ende auf dem Boden einer Kapsel $K$. die ihrerseits mit einer Kernspitze in einem Block $G$ drehbar angebracht ist. Diese Kapsel weitet sich nach oben in einen Kreisring $T$ aus, auf dessen horizontaler glatter Fläche ein Stahlrad $S$ im Kreise um die Achse $C$ läuft. Mit diesem änBeren System, zu dem die Kapsel gehört, schwingt sich ein an ihr angebrachtes Gehäuse $H$ mit herum, in das ein Räderpaar $r_{3} r_{4}$ mit vertikaler Achse eingesetzt werden kann. Das eine Rad $r_{4}$ dieses Paares berührt das Rad $r_{1}$ durch Reibung ${ }^{1}$ ), während das andere tiefer liegende

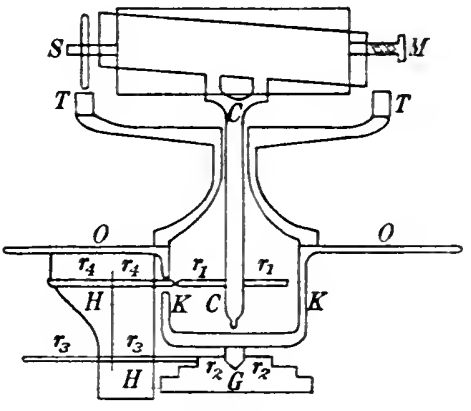

Fig. 7\%. Rad $r_{3}$ dem kreisförmig abgeschliffenen Blocke $G$ mit dem Radius $r_{2}$ gegenüber liegt.

Wird dem äuBeren System eine Drehbewegung um die Konstruktionsachse um einen Winkel $\varphi$ erteilt, so legt der Berïhrungspunkt des Rades $r_{3}$ den $W_{\text {eg }} r_{2} \varphi$ am kreisförmigen Rande des Blockes $G$ zurück, wobei $r_{3}$ eine Drehung um den Winkel $\chi$ erfăhrt und $r_{3} \chi=r_{2} \varphi$ ist. Mithin dreht sich das auf derselben Achse mit $r_{3}$ sitzende Rad $r_{4}$ ebenfalls um $\chi$, und ein Punkt seines Umfangs beschreibt den $\mathrm{Weg}_{4} r_{4}$. Da aber außerdem das ganze äuBere System sich gleichzeitig um den Winkel $\varphi$ um die Konstruktionsachse gedreht hat, so beschreibt der Berührungspunkt des Rades $r_{4}$ mit dem Rade $r_{1}$ in ganzen den Bogen $r_{4} \%+r_{1} \varphi$, und diesem Bogen ist der von dem Berührungspunkte des Rades $r_{1}$ infolge der Reibung zurückgelegte Weg gleich:

$$
r_{1} \psi=r_{4} \%+r_{1} \varphi,
$$

1) Es sind nirgends Zahnräder, sondern ülerall Reibungeräder zur Anwendung gekommen. 
mithin ist

$$
\psi=\frac{r_{4}}{r_{1}} r_{3} r_{3} \varphi+\varphi=\left(1+\frac{r_{2}}{r_{1}} \frac{r_{4}}{r_{3}}\right) \varphi .
$$

Bei der Drehung $\psi$ des inneren Systems und damit auch des Zylinders um die Achse $C$ läuft das Stahlrad $S$ auf dem Ringe, und wenn $R$ der Radius dieses Ringes ist, so legt sein Berührungspunkt den Weg $R(\psi-\varphi)$ zurück, da ja der Ring sich mit dem äußeren System um dieselbe Achse dreht. Das Rad wird dabei den Bogen $S \vartheta$ abwickeln. Nun sitzt $S$ auf der Umdrehungsachse des Zylinders, der daher um denselben Winkel $\vartheta$ rotiert. Er wird durch die Gleichung bestimmt:

$$
\vartheta=\frac{R}{S}(\psi-\varphi)=\frac{R}{S} \frac{r_{2} r_{4}}{r_{1} r_{3}} \varphi .
$$

Wird umgekehrt dem Zylinder eine Drehung um seine Umdrehungsachse um den Winkel $\vartheta$ erteilt, so kann man die Dimensionen der Räder so wählen, daß die horizontale Drehung des Zylinders um die Konstruktionsachse

$$
\varphi=\frac{S}{R} \frac{r_{1}}{r_{2}} \frac{r_{3}}{r_{4}} \vartheta=k \vartheta
$$

wird. Auch läBt sich $R$ durch eine mikrometrische Einstellung $M$ innerhalb enger Grenzen ändern. Für die verschiedenen Koeffizienten werden andere Räderpaare $r_{3} r_{4}$ eingesetzt und statt $r_{2}$ sind im ganzen drei Radscheiben vorhanden, aus denen sich der Block $G$ terrassenförmig aufbaut.

52. Analysator von Michelson und Stratton. Der Analysator von Michelson und Stratton (Fig. 78) dient den beiden Aufgaben, die Kurve zu einer gegebenen Fou rierschen Reihe zu zeichnen und zu einer gegebenen Kurve die Koeffizienten zu finden.

Eine Achse $D$ trägt $n(=80)$ Zahnräder, denen $n(=80)$ Exzenter $A$ gegenüberstehen, in die sie eingreifen. Wir betrachten (s. Fig. 79) nur ein Zahnrad und einen Exzenter, der bei einer Umdrehung der Achse $x$ Umdrehungen machen möge. Die Bewegung des Exzenters wird durch den Hebel $B$ auf die Stange $R$ und auf den Punkt $x$ übertragen. Dabei ist die Länge $d$ des Hebelarms regulierbar. Die auf- und abgehende Bewegung von $x$ ist dann proportional $z_{\varkappa}=d_{\varkappa} \sin \varkappa \vartheta$, wenn $\vartheta$ der Drehungswinkel des Zahnrads ist. Jeder der 80 Punkte $x$ ist durch je eine, bei allen gleiche Spiralfeder $s$ mit je einem Hebelarm $u$ verbunden, der an 
einem langen Hohlzylinder $C$, der auf zwei Schneiden ruht, senkrecht zu dessen Achse angebracht ist. Durch eine andere Feder $S$, welche an einem gegenüberstehenden Hebelarm wirkt und den sämtlichen Federn $s$ das Gleichgewicht hält, wird die Drehberegung des Zrlinders gedämpft. Der Hebel u trägt einen Draht $u$ : durch den die Bewegung des $\mathrm{Zy}$ linders auf eine Schreibfeder übertragen wird, welche auf einer tafelförmigen Scheibe die Kurre aufzeichnet. Diese Scheibe rerschiebt sich bei einer vollen Umdrebung ron $D$ um das Abszissenintervall $u$.

Sind $l$ und $L$ die natürlichen Längen der Federn $s$ und $S$ und werdenihnen durch Drehungder Zahnradachse Verlängerungen $x$

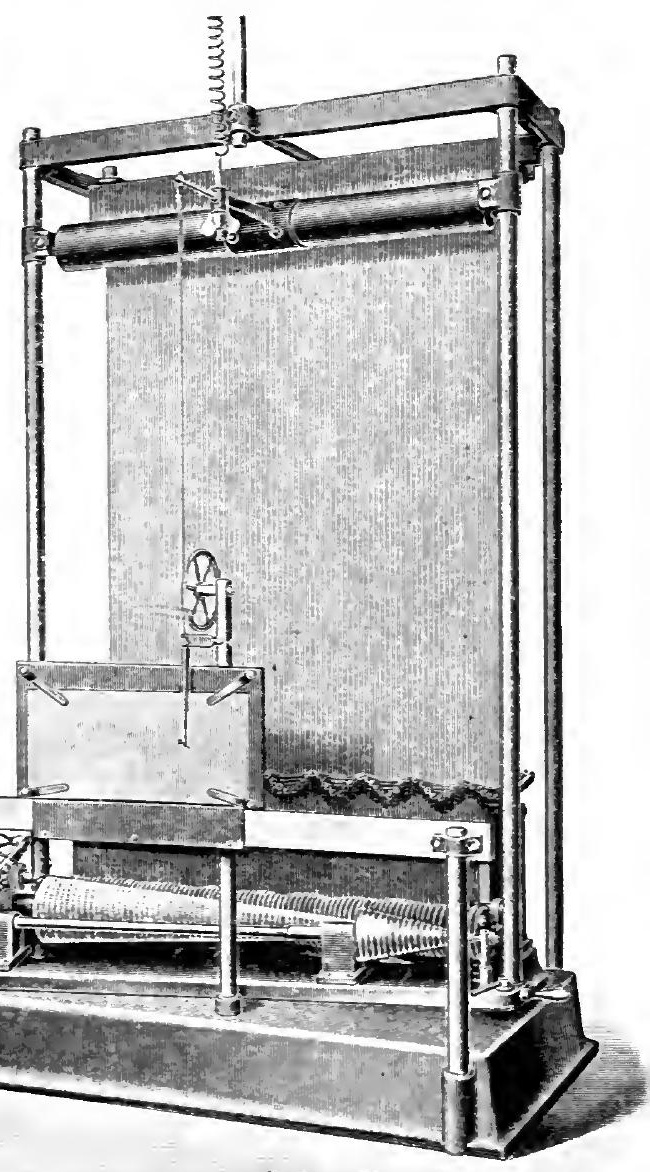
bzw. $y$ erteilt, so sind ibre \%ughriitte

$$
p={ }_{l}^{e}(l+i) \quad P={ }_{L}^{E}(L+l),
$$

worin $t$ und $E$ die Kräfte für ihre natürlichen Lïngen sind $x$, $=0$, $y=0$ ). Ist $u$ der Hebelarm der kleinen Federn, b ler Hebelarm 
der großen Feder, so wird Gleichgewicht eintreten, wenn $a \Sigma p=b P$ ist oder

$$
a \frac{e}{l} \sum\left(l+x_{\varkappa}\right)=b \frac{E}{L}(L+y) \text {. }
$$

Hierbei setzt $\operatorname{sich} x_{\chi}$ aus zwei Teilen zusammen, der Verschiebung $z_{z}$ des Aufhängungspunktes der Feder und der durch die Wirkung der großen Feder bewirkten Verkürzung $\xi$, die proportional der Verlängerung $y$ der großen Feder gesetzt werden kann, so daB

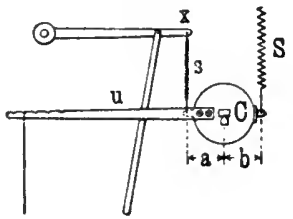

$$
\xi=\frac{a}{b} y
$$

wird. Dann folgt

$a \sum^{e} \sum_{x=1}^{n}\left(l+z_{\varkappa}-\frac{a}{b} y\right)=b \frac{E}{L}(L+y)$.

Im Anfangszustand ist $n a e=b E$, also

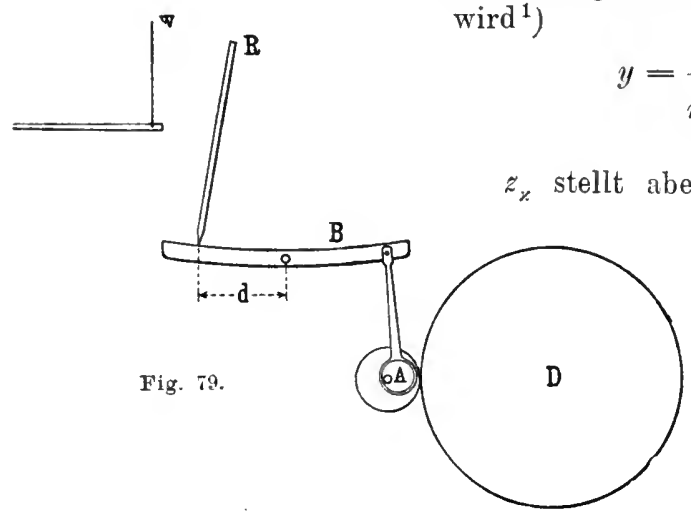

$$
y=\frac{\Sigma z_{\varkappa}}{n\left(\frac{l}{L}+\frac{a}{b}\right)} .
$$

$z_{\varkappa}$ stellt aber die harmonische Bewegung des' Punktes $x$ dar: $z_{x}=d_{x} \sin \varkappa \vartheta$ Wenn man also $d_{x}=\alpha_{x}$ macht, so erhält man für $x=1, \ldots n: \Sigma z_{x}=$ $\Sigma a_{x} \sin \psi \vartheta$, die Summe der sinus-Glieder der Fourierschen Reihe. Macht man die Achse $D$ mit ihren einen Konus bildenden Zahnrädern frei, so kann man mit Hilfe eines langen Getriebes alle Exzenter auf einmal um $90^{\circ}$ drehen und erhält dann, wenn man $d_{x}=b_{x}(x=1 \ldots n)$ macht,

1)

$$
\frac{1}{n}\left(\frac{\Sigma l}{l}+\frac{\Sigma z_{k}}{l}-\frac{n}{l} \frac{a}{b} y\right)=\frac{L+y}{L} .
$$

Hier ist $\Sigma l=n l$, also

$$
\frac{\Sigma z_{\varkappa}}{n l}-\frac{1}{l} \frac{a}{b} y=\frac{y}{L} \quad \text { und } \quad y\left(\frac{l}{L}+\frac{a}{b}\right)=\frac{\Sigma z_{x}}{n} .
$$


$\Sigma z_{x}{ }^{\prime}=\Sigma b_{x} \cos \% \vartheta$. Man erhält auf einmal entweder nur eine sinusoder eine cosinus-Reihe.

$y$ ist aber $\Sigma z_{x}$ proportional, und die Bewegung des Zeichenstiftes ist mit $y$ proportional, mithin erhält man bei passenden $\mathrm{Ab}$ messungen des Apparats das Bild der durch $a_{x}$ oder $b_{\%}$ gegebenen Kurve.

Soll dagegen eine gegebene Kurve analysiert werden, so nimmt die Aufgabe im vorliegenden Falle folgende Form an: Es sind $a_{*}$ und $b_{x}$ zu bestimmen aus den Gleichungen (5) S. 133:

$$
n a_{*}=\sum_{i=1}^{2 n} y_{\lambda} \cos \% \theta_{\lambda}, \quad n b_{*}=\sum_{i=1}^{2 n} y_{i} \sin \% \theta_{i}, \quad \%=1 \ldots n,
$$

wobei $\theta_{2}=\frac{2 \pi x_{\lambda}}{u}$ ist. Wir teilen das Abszissenintervall $u$ der gegebenen Kurre in $2 n=80$ gleiche Teile, so daB

$$
\frac{x_{\lambda}}{u}=\frac{\lambda}{80} \text { und } \theta_{i}=\frac{2 \pi \lambda}{80}\left(\lambda_{0}=1,2 \ldots 80\right)
$$

wird und errichten in den Teilpunkten die Ordinaten $y_{\lambda}$. Diesen machen wir die 80 Hebelarme gleich: $d_{r}=y_{2}$ und zeichnen durch Drehung der Kurbel der Zahnräderwelle $D$ die zugehörigen Kurven, deren Ordinaten wir zum Unterschied mit $z$ und $z^{\prime}$ bezeichnen. Wir erhalten dann entsprechend den Gleichungen (4) S. 133:

$$
z_{2}=\sum_{x=1}^{80} d_{x} \cos \left(x \lambda \frac{2 \pi}{80}\right), \quad z_{i}^{\prime}=\sum_{x=1}^{80} d_{x} \sin \left(x \lambda \frac{2 \pi}{80}\right) .
$$

Hier können wir offenbar $\%$ und $\lambda$ vertauschen, also auch schreiben:

$$
z_{\lambda}=\sum_{i=1}^{80} y_{\lambda} \cos \left(* \lambda \frac{\pi}{40}\right), \quad z_{i}^{\prime}=\sum_{i=1}^{80} y_{\lambda} \sin \left(x_{\lambda} \frac{\pi}{40}\right)
$$

d. h. es ist $z_{i}=40 a_{x}, z_{\lambda}^{\prime}=40 b_{x}$. Wenn wil also für die zuletzt erbaltenen Kurven das einer vollen Periode entsprechende Abszissenintervall wiederum in 80 Teile teilen, so ist die zum $\lambda^{\text {ten }}$ Teilpunkte gehörige Ordinate $z_{\lambda}$ bzw. $z_{i}^{\prime}$ das 40 fache des gesuchten Koeffizienten $a_{x}$ bzw. $b_{x}$.

Dieser Apparat wird seine Anwendung finden, wenn es nicht auf äuBerste Genauigkeit ankommt, sondern Fehler vou 1 bis $2 \%$ gestattet sind. Dafür liefert er in schnellster Weise die Kurven mit 
Berücksichtigung der Glieder vom 80 fachen Winkel und es ließe sich diese Zahl noch leicht vermehren.

53. Apparat von Terada zur Analyse und Synthese von Schwingungen. Der Analysator von Terada, der zwar auch zur Analyse von gegebenen Kurven benutzt werden kann, dient in erster Linie zwei anderen Aufgaben, nämlich erstlich zu einer gegebenen Kurve, für deren Grundschwingung Amplitude und Periode bekannt sind (so daß man sie an den Skalen des Apparates einstellen kann), die Kurve der Oberschwingungen zu zeichnen und zweitens aus den einzelnen gegebenen Schwingungen die

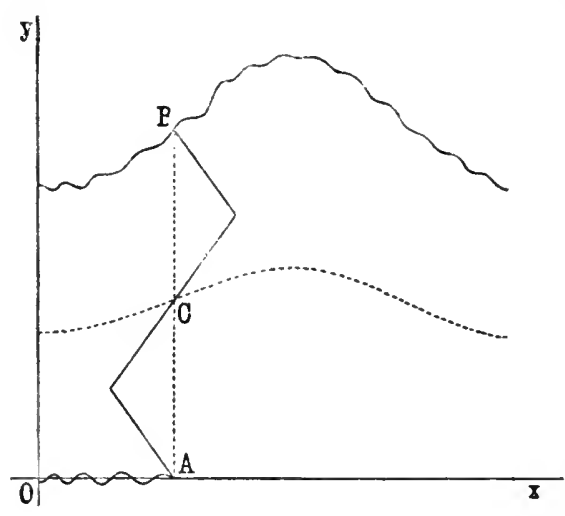

Fig. 80. zusammengesetzte Kurve darzustellen.

Wir denken uns zunächst eine vom Punkte $B$ (Fig. 80 ) durchlaufene Kurve gegeben und für diesen Punkt die Ordinate $B A$ gezogen, dann wird ein dreiteiliges Gelenksystem, welches in $B$ und $A$ Führungs- bzw. Zeichenstifte trägt, entlang der Kurve geführt und der Apparat sorgt dafür, daB $B A$ stets mit den Ordinaten von $B$ zusammenfällt. Dem Mittelpunkte $C$ des Gelenksystems wird nun eine vorgeschriebene harmonische Bewegung (Sinuskurve) mit Hilfe des Apparates erteilt. Da die Dreiecke, die das Gelenksystem mit der Ordinate bildet, gleichschenklig sind, so sind sie mit Rücksicht auf die Gleichheit der Winkel bei $C$ auch in jedem Augenblicke kongruent.

Sind also in den drei Kurven die Ordinaten

$$
\begin{aligned}
& \text { des Punktes } B \\
& y_{1}=b_{0}+b_{1} \sin \vartheta+\sum_{x=2}^{\infty} b_{x} \sin x \vartheta \\
& " \quad \text { " } \quad y_{2}=\frac{b_{0}}{2}+\frac{b_{1}}{2} \sin \vartheta \\
& \text {, }, \quad A \quad y_{3},
\end{aligned}
$$

so ist wegen 


$$
\begin{gathered}
y_{3}-y_{2}=y_{2}-y_{1} \\
y_{3}=2 y_{2}-y_{1}=-\sum_{x=2}^{\infty} b_{x} \sin x \vartheta
\end{gathered}
$$

d. h. der Stift $A$ zeichnet das Spiegelbild der Oberschwingungen. Man kann das Verfabren wiederholen und andere Oberschwingungen eliminieren, indem man z. B. bei $C$ das Glied mit $\sin 2 \vartheta$ hinzunimmt. Auch kann man eine MaBstabänderung für $y_{3}$ erbalten, wenn man $B C: C A=1: \mu$ macht, und dasselbe Verhältnis entsprechender Seiten der beiden Dreiecke durch Verschieben der Gelenke herstellt, ferner dem Punkte $C$ die Bewegung

$$
y_{2}=\frac{\mu}{\mu+1}\left(b_{0}+b_{1} \sin \vartheta\right)
$$

erteilt. Dann wird wegen

also

$$
\begin{aligned}
& y_{3}-y_{2}=\mu\left(y_{2}-y_{1}\right) \\
& y_{3}=(\mu+1) y_{2}-\mu y_{1},
\end{aligned}
$$

$$
y_{3}=-u \sum_{*=2}^{\infty} b_{\%} \sin \% \vartheta .
$$

Der Apparat besteht aus zwei Zeichenblöcken (Fig. 81), welche die in derselben Ebene liegenden Blätter für die ron $A$ und $B$ beschriebenen Kurven tragen. Zwischen beiden befindet sich ein $\mathrm{Wa}$ gen $K$, der sich auf Schienen bewegt, die der $x$-Achse parallel lanfen. Dieser Wagen trägt einen Konus $W$, dessen Spitze nach unten gerichtet ist und der an der einen verstellbaren Schiene $R_{1}$ anliegt. Je nachdem diese Schiene, den Konus berührend, hinauf oder hinunter geschoben wird, erzielt man eine schnellere oder langsamere Undrehung des Konus bei Berregung des Wagens. Die Einstellung dieser Schiene wird daher die Periode bestimmen, in der sich ein oben auf dem Konus befindlicher Stift $P$ um die Achse des Konus herumdreht. Dieser Stift kann nun in rerschiedene Entfernung von der Achse gestellt werden, wodurch die Amplitude der durch ihn hervorgerufenen Bewegung des Punktes $C$ bestimmt wird. Dieser Stift wird nämlich in dem der $x$-Achse parallelen Schlitz eines in der Richtung der $y$-Achse beweglichen Schlittens $S$ geführt. Bei der Drehung des Stiftes um die Konusachse führt der Schlitten die Verschiebungen aus, welche die Pro- 
jektion des Punktes $P$ auf die $y$-Achse erfährt, und da der Punkt $C$ mit ihm fest verbunden ist, so führt $C$ ebenfalls eine harmonische Bewegung in der Richtung der $y$-Achse aus. Die Stifte $A$ und $B$ werden auch in der Richtung der $y$-Achse durch Schienen geführt, die mit dem Wagen fest verbunden sind. Die Gelenkver-

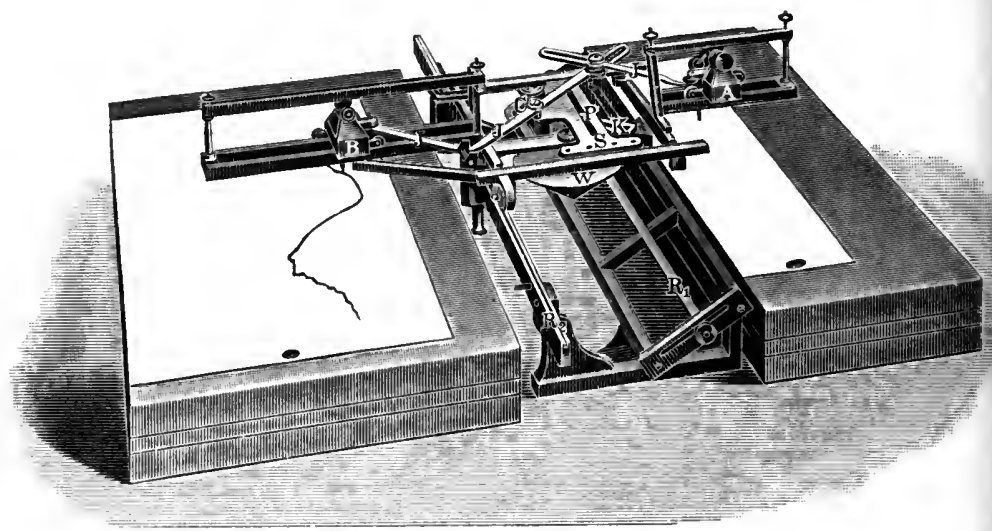

Fig. 81 .

bindung $J J$ besteht in der oben beschriebenen Weise zwischen $B, C$ und $A$. Die Gelenke selbst werden durch Schrauben gebildet, die durch Schlitze geführt und in diesen verstellbar sind.

Die zweite der genannten Aufgaben läßt sich folgendermaßen lösen. Jan führt $A$ auf einer Parallelen zur $x$-Achse, dann beschreibt $B$ eine Sinuslinie (deren Amplitude und Periode an der Schiene $R_{1}$ und dem Stifte $S$ eingestellt werden muB). Man bringt diese Sinuslinie unter $A$ und stellt Amplitude und Periode der ersten Oberschwingung ein, so erhält man unter $B$ die aus beiden Schwingungen zusammengesetzte und kann in dieser Weise fortfahren.

\section{Abschnitt.}

\section{Integraphen.}

54. Grundlagen. Bei den in den voranstehenden Abschnitten behandelten Integrierapparaten wurden bestimmte Integrale zwischen gegebenen Grenzen erhalten, die Integraphen geben auch unbestimmte Integrale, indem sie, und dies ist ihr wesentlicher Unterschied, die Integralkurve graphisch aufzeichnen. 
Wir nehmen an, die gegebene Kurve, die wir als Differentialkurve bezeichnen, habe die Gleichung $y=f(x)$, dann bezeichnen wir als Integralkurve die durch die Gleichung

$$
Y=\int f(x) d x+C=F(x)+C
$$

definierte Kurve.

Wir denken uns beide Kurven auf rechtwinklige Koordinatensysteme mit derselben $y$-Achse und parallelen $x$-Achsen, die um $C$ von einander abstehen, bezogen $\left(\mathrm{O}_{1} \mathrm{O}_{2}=C\right)$. Die Gleichung für $Y$ zeigt, wenn wir das Integral zwischen bestimmten Grenzen (z. B. $x_{1}$ für den Punkt $O_{1}$ und $x_{2}$ für den Punkt $C$ ) nehmen, daB die Ordinate $(B C)$ eines Punktes $(B)$ der Integralkurve den Inhalt der von der Differentialkurve, demselben Abszissenintervall und den zugehörigen Ordinaten begrenzten Fläche mißt. Wir wählen eine passende Strecke in der $x$-Achse als Längeneinheit, z. B. $C D$ und tragen sie von dem OrdinatenfuBpunkt $C$ der Punkte $A$ und $B$ nach rückwärts ab, dann schlieBt die Verbindungslinie

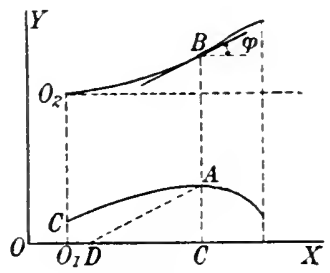

Fig. 82 . $D A$, die wir die Direktrix der Kurve $f(x)$ im Punkte $A$ nennen, mit der $x$-Achse einen Winkel $\varphi$ ein, der durch tg $\varphi=y$ bestinmt ist, da ja $D C=1$ angenommen ist.

Andererseits ist $\frac{d Y}{d x}=f(x)=y=\operatorname{tg} \varphi$, d. h. die Tangente der Integralkurve in einem Punkte $B(x, Y)$ schlieBt mit der $x$-Achse denselben Winkel $\varphi$ ein und ist daher der Direktrix in dem Punkte $A$, der auf der Ordinate von $B$ liegt, parallel. Diese Eigenschaft kommt sämtlichen Integralkurven zu, deren es wegen der willkürlichen Konstante $C$ unendlich viele gibt. Denkt man sich das Koordinatensystem so verschoben, daB der Anfangspunkt der Differentialkurve mit dem Koordinatenanfange zusammenfällt, so daß also in diesem Punkte $y=0$ ist, so wird $Y=C$, und damit ist für einen gegebenen Wert von $C$ die Lage der Integralkurre bestimmt. Ferner wird für jeden Schnittpunkt der Differentialkurve mit der $x$-Achse $y=0$ und damit $\varphi=0$, mithin ist in dem zu derselben Abszisse gehörenden Punkte der Integralkurve die Tangente der $x$-Achse parallel, und die Bedingung $\frac{d Y}{d x}=0$ drückt gleichzeitig 
aus, dab die Ordinate der Integralkurve ein Maximum oder Mini mum erreicht.

Erreicht die Ordinate der Differentialkurve ein Maximum ode Minimum, so ist $\left|\frac{d y}{d x}\right|_{x_{0}}=0$. Dann wird $\left|\frac{d^{2} Y}{d x^{2}}\right|_{x=x_{0}}=0$, also hat die Integralkurve im allgemeinen einen Wendepunkt.

Ist ein Stück der Differentialkurve eine Parallele $y=b$ zu $x$-Achse, so ist $\frac{d Y}{d x}=\operatorname{tg} \varphi=b$ und $Y=b x+c$ die Gleichung des entsprechenden Stückes der Integralkurve, die eine unter dem Winkel $\varphi=\operatorname{arctg} b$ geneigte Gerade darstellt. Ist umgekehrt die Differentialkurve eine geneigte Gerade $y=a x+b$, so ist $Y=$ ${ }_{2}^{a} x^{2}+b x+c$, also die Integralkurve eine Parabel. Wenn mar daher die Differentialkurve aus kleinen geraden Linienelementer entstehen läBt, also als einen Polygonzug ansieht, so wird die danach gezeichnete Integralkurve sich aus kleinen Parabelelementer zusammensetzen.

Ist die Differentialkurve ein Kreis, mit der Gleichung

so ist

$$
(x-a)^{2}+(y-b)^{2}=r^{2},
$$

$$
\frac{d y}{d x}=\begin{aligned}
& a-x \\
& y-b
\end{aligned}
$$

Für die Punkte $x=a, y=b \pm r$ wird $\left|\frac{d^{2} Y}{d x^{z}}\right|_{x=a}=0$. Die Integralkurve hat jedesmal einen Wendepunkt, wenn die Tangente der Differentialkurve der $x$-Achse parallel ist. Wenn die Tangente der Differentialkurve der $y$-Achse parallel ist, was in den Punkten $y=b, x=a \pm r$ stattfindet, so wird

$$
\frac{d y}{d x}=\infty, \varphi=90^{\circ} \text { und } \quad\left|\frac{d^{2} Y}{d x^{2}}\right|_{x=a \pm r}=\infty ;
$$

in diesem Falle hat die Integralkurve eine Spitze. Es sei noch bemerkt, da $B$ die Integralkurve keine Strecken enthält, die der $y$-Achse parallel sind, weil für $\frac{d Y}{d x}=\infty$ auch $y=\infty$ sein würde. ${ }^{1}$ )

1) D'O cag ne gibt eine einfache Konstruktion für den Krümmungsradius $r$ der Integralkurve an. Es seien $A$ und $B$ die derselben Abszisse entsprechenden Punkte der Differential- und Integralkurve, 
55. Instrument von Abdank-Abakanowicz. Zuerst hat das Prinzip eines Integraphen Coriolis angegeben (1836), später haben sich Zmurko (1861), J. Thomson und Cayley (1876 und 1877) damit beschäftigt, 1878 haben $\mathrm{Abdank}-\mathrm{Abakanowicz}$ und ziemlich gleichzeitig Boy s Integraphen konstruiert. Wir gehen nur auf die nenere Form ein, welche Coradi dem Instrument ron Abdank-Abakanowicz gegeben bat, dessen Konstruktion der Erfinder nicht mehr erlebt hat.

Das Instrument besteht a us einem rechteckigen Rahmen, dessen lange Seiten $L$ und $L^{\prime}$ (Fig. 84) bei dem größeren Modell etwa 52, bei dem kleineren $27 \mathrm{~cm}$ lang sind und als Schienen für zwei Wagen, den Differentialwagen $W$ und den Integralwagen $W^{1}$

$A T$ die Tangente der Differentialkurve und $C T=\sigma$ ihre Subtangente, $\varphi$ wie früher der Winkel der Tangente $B E$ der Integralkurre mit der $x$-Achse oder auch der Direktrix $D A$ der Differentialkurre, so ist $\sigma=y \frac{d x}{d y}$ oder wegen $\frac{d Y}{d x}=\operatorname{tg} \varphi=y$ auch $\sigma=\operatorname{tg} \varphi \frac{d x}{d y}$. Wenn $d S$ das Bogenelement der Integralkurre bezeichnet, ist $r=\frac{d S}{d \varphi}$ und $\cos \varphi d S=d x$. Da ferner $\sec ^{2} \varphi d \varphi=d y$ ist, so ergibt sich

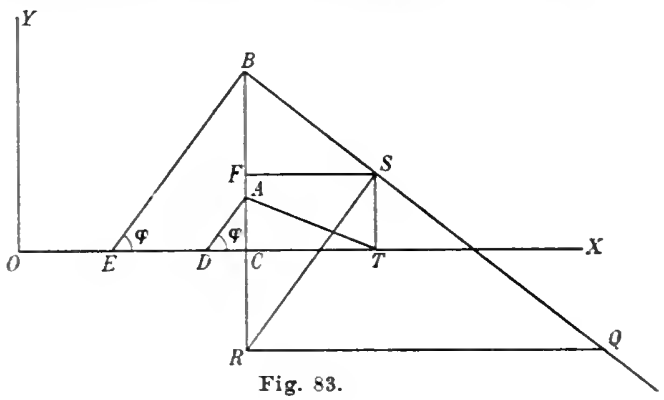

$$
r=\frac{d S}{d y} \sec ^{2} \varphi=\frac{d x}{d y} \sec ^{3} \varphi=\frac{\sigma}{\sin \varphi \cos ^{2} \varphi} \text { oder } \sigma=r \sin \varphi \cos ^{2} \varphi .
$$

Man errichte in $B$ senkrecht zur Tangente $B E$ die Normale der Integralkurre, welche in $S$ die Ordinate ron $T$ schneide. In $S$ errichte man wiederum eine Senkrechte $S R$ auf $S B$, deren Schnittpunkt mit der Ordinate $C B$ in $R$ sei. Eine Parallele $R Q$ zur $x$-Achse trifft die Normale $B S$ im Punkte $Q$, dem gesuchten Krümmungsmittelpunkt. In der Tat ist, wenn wir noch die Parallele $F S=\sigma$ ziehen,

also

$$
B F=\sigma \operatorname{ctg} \varphi, \quad F R=\sigma \operatorname{tg} \varphi,
$$

und

$$
B R=\sigma(\operatorname{tg} \varphi+\operatorname{ctg} \varphi)
$$

$$
B Q=\frac{B R}{\cos \varphi}=\frac{\sigma}{\sin \varphi \cos : \frac{1}{\varphi}} \text {. }
$$


dienen. Der Rahmen selbst ruht auf zwei an seinen beiden schmalen Enden angebrachten Rollen $r$, während als dritter Stützpunkt ein mit dem Differentialwagen verbundener Fahrstift $t$ bzw. eine seitlich neben ihm angebrachte Stütze $s$ dient. Das Instrument hat also insoweit eine gewisse Ähnlichkeit mit dem Coradischen Rollplanimeter (vgl. Fig. 50 u. 53). Die Bewegung auf den Rollen findet senkrecht zur Schienenrichtung in gerader Linie statt, die wir als Abszissenrichtung wählen. Jeder der beiden auf den Schienen laufenden Wagen wird auf zwei hintereinander in einer Nut rollenden Rädchen bewegt. Während der Differentialwagen, wie erwähnt, an einem in der Länge innerhalb gewisser Grenzen verstellbaren, senkrecht zur Schiene gerichteten Arme $B$ den Fahrstift trägt, ist mit dem Integralwagen ein Zeichenstift $k$ verbunden. Der Arm $A$ desselben ist unter den Schienen nach vorn durchgeführt, so daß dieser Stift, ebenso wie der andere von dem Beobachter im Auge behalten wird. Auch dieser Arm hat die Richtung der $x$-Achse. Mit dem Integralarm ist nun in einem um eine senkrechte Achse drehbaren Rahmen $C$ die scharfkantige Rolle $i$ verbunden, welche auf der Zeichenebene rollend die Integralkurve beschreibt. Damit dieses Rad in jedem Augenblick die Richtung der Tangente der Integralkurve einnimmt, ist folgende Einrichtung getroffen. Um eine in der Mitte des Instruments befindliche feste Achse ist eine Schiene $D$ drehbar, und zwar so, daß sie sich in einem Lager $g$, das mit dieser Achse verbunden ist, frei hin- und herschieben läBt. Ihr eines Ende ist an einem Punkte $M$ des Differentialwagens drehbar befestigt, von dem wir zunächst annehmen wollen, daB er auf der Differentialkurve geführt wird. (Er entspricht also dem Punkte $A$ in Figur 82, während der Drehpunkt der Schiene in der Mitte des Instruments den Punkt $D$ darstellt, die Abszissendifferenz beider Punkte ist konstant und stellt die als Einheit gewählte Strecke $C D$ dar.) Auf der Direktrixschiene $D$ läuft nun ein dritter Wagen $W^{2}$ und dieser ist mit dem Rahmen, der die Achse der Rolle $i$ hält, durch ein Gelenkparallelogramm $P$ verbunden. Hierdurch wird die Ebene von $i$ stets der Schiene $D$ und der Bewegungsrichtung des Wagens $W^{2}$ parallel gehalten.

Dadurch, daB nicht der Drehungspunkt $M$ der Direktrixschiene am Wagen $W$, sondern der senkrecht zur Bewegungsrichtung von $W$ stehende Differentialarm den Fahrstift trägt, tritt eine Verschiebung der $y$-Achse der Differentialkurve ein. Ebenso zeichnet nicht die Rolle $i$, sondern der um eine konstante Länge in der 
Richtung der $x$-Achse abstehende Zeichenstift $k$ die Integralkurre, wodurch auch diese in der Abszissenrichtung rerschoben erscheint. Eine solche Verschiebung wird ferner dadurch notwendig, daB die beiden Stifte $t$ und $k$ sich nicht gegenseitig stören dürfen. Bringt man nämlich den Differentialwagen $W$ in die Mitte des Instruments, so da B die Direktrixschiene parallel der $x$-Achse steht, so rollt der Wagen $W^{2}$ in der Richtung der $x$-Achse und auch die Rolle $i$ muB in dieser Richtung rollen. Um dies aber' zu ermöglichen, wird der Wagen $W^{1}$, mit dem die Rolle $i$ fest verbunden ist, gezwungen, ebenfalls in die Mitte des Instruments zu rollen. Folglich befinden sich nicht nur $i$ und $W^{-2}$ in derselben Tertikalebene (die senkrecht zur Längserstreckung des Instruments steht), sondern auch die beiden Stifte. Der Differentia'stift $t$ ist deshalb an einem etwas höher liegenden Arm angebracht und dieser Arm kann durch die Hülse $H$ etwas rerlängert werden, damit die beiden Stifte sich nebeneinander befinden (um eine konstante A bszissendifferenz ron einander liegend). In dieser Grundstellung beschreiben bei Berregung des ganzen Instruments die beiden Stifte eine der $r$ Achse parallele Gerade. Man untersucht auf diese Weise ror Beginn der Arbeit mit dem Instrument. ob die Schienen die rorschriftsmåBige Lage parallel zur $y$-Achse haben.

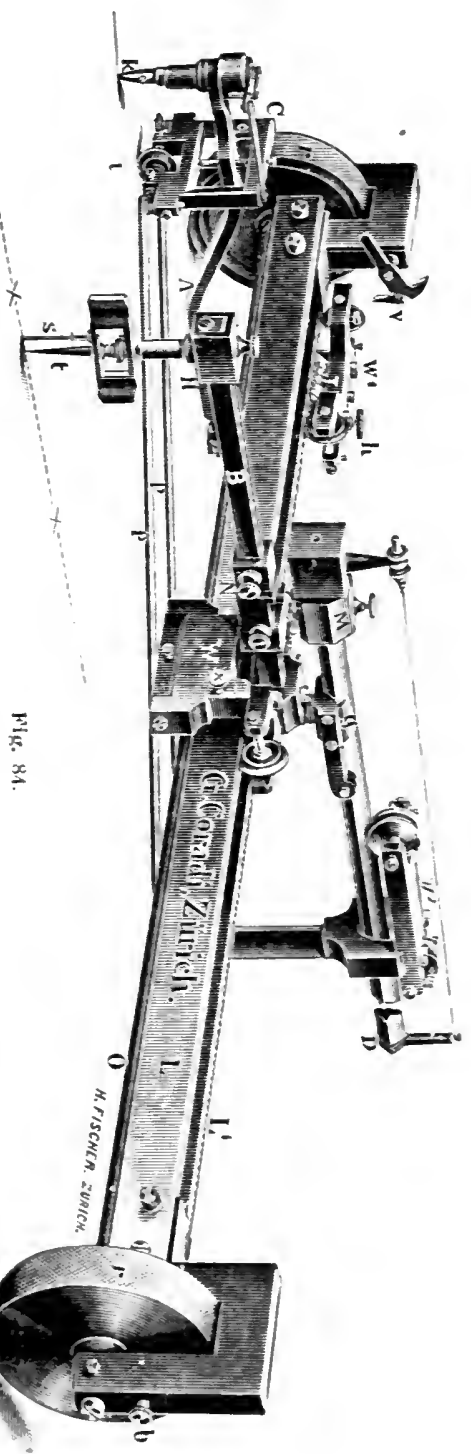


Will man eine vorgelegte Kurve integrieren, so löst man den Differentialwagen $W$, den man an jeder Stelle festklemmen kann, und bringt den Fahrstift $t$ auf den Anfangspunkt der Kurve. Den Integralwagen $W^{1}$ schiebt man dagegen möglichst weit zur Seite, wo die Teilung ihren Anfangspunkt hat, an der man die Ordinaten $Y$ ablesen kann. Wie weit diese Verschiebung möglich ist, hängt von der Längeneinheit ab, die sich in gewissen Grenzen verändern läBt ${ }^{1}$ ), beim großen Apparat zwichen 10 and $20 \mathrm{~cm}$, beim kleinen zwischen 4 und 12,5 cm. Jede Bewegung des Fahrstifts $t$ in der Richtung der $x$-Achse wird durch das Rollen des ganzen Instrumentes auf den Rädern $r$, jede Bewegung in der $y$-Achsenrichtung durch Verschiebung des Differentialwagens $W$ auf seiner Schiene $L$ bewirkt. Bei letzterer Bewegung wird die Direktrixschiene um den festen Drehpunkt (unterhalb $g$ ) in der Mitte des Instruments gedreht. Die Einstellung des Stiftes $t$ bzw. des Wagens $W$ in der $y$-Richtung bestimmt also die Richtung der Direktrixschiene $D$. Der Wagen $W^{2}$ bleibt dabei durch seine Verbindung mit dem Rahmen $C$ der Integrierrolle nahezu in unveränderter Entfernung von diesem und rollt infolgedessen auf der Schiene $D$ bei ihrer Drehung. Das von $W^{2}$ geführte Gelenkparallelogramm $P$ drebt nun den Rahmen $C$ so, daB die Ebene der Rolle $i$ parallel der Schiene $D$ liegt. Wird diese Rolle durch Bewegung des Instrumentes in der Abszissenrichtung in Drehung versetzt, so kann sich ihre Drehungsebene nicht ändern. Dadurch entsteht eine seitliche Komponente, welche die Rolle $i$ und damit den Stift $k$ durch Bewegung des Wagens $W^{\prime}$ längs $L^{\prime}$ in der Ordinatenrichtung verschiebt.

Die Ausdehnung der gegebenen Kurve kann, wie man sieht, in der $x$-Richtung beliebig grob sein, in der $y$-Richtung ist sie durch die Abmessungen des Instruments (auf etwa $52 \mathrm{~cm}$ beim großen Modell) beschränkt. ${ }^{2}$ )

1) Der Differentialarm $B$ trägt eine Teilung. Durch die Schraabe $N$ läBt sich der vordere Drehpunkt der Direktrixschiene $D$ auf dem Differentialarm verstellen, wodurch die Einheitsstrecke geändert werden kann.

2) Die Anwendungen des Instruments sind sebr zallreich. Wir geben nur einige Beispiele:

a) Auflösung numerischer Gleichungen, z. B.:

$$
x^{4}-2,50 x^{3}+1,76 x^{2}-0,14 x-0,12=0 .
$$

Wir setzen 
56. Integrator von Pascal. Der wesentliche Punkt, durch den sich der von Ernesto Pascal 1909 konstruierte Integratore meccanico ron dem vorhergehenden unterscheidet, liegt in dem Wegfall der festen Drehachse der Direktrixschiene. Im übri-

$$
\begin{array}{rlrl}
y & =x^{4}-2,50 x^{3}+1,76 x^{2}-0,14 x-0,12=y_{0} \\
\frac{d y}{d x} & =4 x^{3}-7,50 x^{2}+3,52 x-0,14 & & =y_{1} \\
\frac{d^{2} y}{d x^{9}} & =12 x^{2}-15 x+3,52 & & =y_{9} \\
\frac{d^{3} y}{d x^{3}} & =24 x-15 & & =y_{3} \\
\frac{d^{4} y}{d x^{4}} & =24 & & =y_{4} .
\end{array}
$$

Wir nehmen an, daB die Instrumentalkonstante, d. h. die Zahl, mit der die Ordinate der Integralkurre multipliziert werden muB, um die Fläche der Differentialkurve $z$ wischen der $x$-Achse und der entsprechenden Ordinate zu erhalten, der Einheit gleich sei. Man nimmt zunächst als Differentialkurve die Gerade $y_{4}=24$, dann zeichnet der Integralstift $y_{3}=24 x+C$. Dann stellt man den Differentialwagen so, daB für $x=0 \quad y=-15$ ist und durchläuft die Gerade $y_{3}=$ 24x-15. Man erhält die Integralkurre $y_{2}$. Indem man den Differentialwagen so stellt, daB für $x=0 y=+3,52$ ist, integriert man die erhaltene Kurve $y_{2}$ and so fort, bis man schlieBlich $y_{0}$ erhält. Die Schnittpunkte $(y=0)$ der zuletzt erhaltenen Kurve mit der $x$-Achse liefern die Wurzeln der vorgelegten Gleichung:

$$
x_{1}=-0,2, \quad x_{2}=+0,5, \quad x_{s}=+1,0, \quad x_{4}=+1,2 .
$$

b) Differentiation von Differentialgleichungen $\frac{d^{n} y}{d x^{n}}=f^{n}(x)$, wenn die Gleichung durch eine Kurve gegeben ist. Man erbălt durch den Apparat die $n$ auf einander folgenden Integralkurren.

c) Bestimmung der Momente einer Fläche in bezug auf eine (der $y$-Achse parallele) Achse.

Ist $\int_{0}^{a} y d x$ die gegebene Fläche, so ist $Y_{1}=\int_{0}^{x} y d x$ die Fläche der vorgelegten Kurve bis zur Ordinate in $x$,

$$
Y_{2}=\int_{0}^{a} Y_{1} d x=\int_{0}^{a} \int_{0}^{x} y d x \cdot d x=\int_{i}^{a} x y d x
$$

das statische Moment der Fläche in bezug auf eine mit der Endordinate $(x=a)$ zusammenfallende Momentenachse. Ferner ist 
gen ist auch hier ein paralleles Schienenpaar auf einem Rahmen vorhanden, der senkrecht zur Schienenrichtung, die wir der $y$-Achse parallel annehmen, auf zwei Rollen läuft. Auf der einen Schiene läuft der Differential-, auf der anderen der Integralwagen. Die Direktrixschiene verbindet hier einfach die beiden Wagen und zwar ist sie um eine Vertikalachse des Differentialwagens so drehbar, daß sie sich in einem mit dieser Achse sich drehenden Lager in ihrer Längsrichtung verschiebt. Sie trägt am hinterer Ende (Fig. 85) den Rahmen für die Achse der Integrierrolle, deren Ebene der Direktrix par-

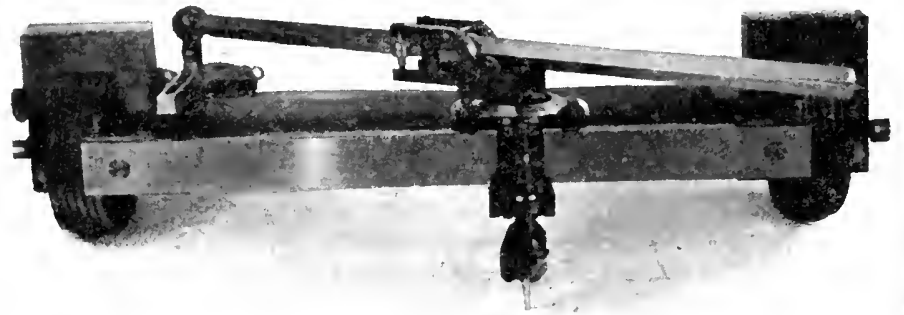

Fig. 85 .

allel gehalten wird. Der Abstand der Schienen oder genauer die Projektion der Entfernung vom Drehpunkt der Direktrix bis zum Berührungspunkt der Integrierrolle mit der Papierebene auf die $x$-Achse ist die Längeneinheit oder Basis des Apparates. Mit den

$$
Y_{3}=\int_{0}^{a} Y_{2} d x=\int_{0}^{a} y d x \int_{0}^{x} x d x=\int_{0}^{a} \frac{x^{2}}{2} y d x .
$$

Also liefert die Ordinate $Y_{3}$ der dritten Integralkurve das Trägheitsmoment in bezug auf dieselbe Achse. Auf einfache Weise läßt sich auch die Lage des Schwerpunktes der Fläche bestimmen.

d) Wahrscheinlichkeitsrechnung. Trägt man die Fehler $\varepsilon$ einer Beobachtungsreihe als Abszissen auf, die relative Häufigkeit (Anzahl dividiert durch Gesamtzahl der Fehler) als Ordinaten und bezeichnet diese mit $y=\varphi(\varepsilon)$, so wird die Wahrscheinlichkeit des Fehlers durch das Integral $W=\int \varphi(\varepsilon) d \varepsilon$ dargestellt. Wenn man die Kurve $\varphi(\varepsilon)$ mit dem Fahrstift durchläuft, so geben die Ordinaten der Integralkurve $W$.

Bezüglich anderer Anwendungen sei auf Br. Abdank-Abakanowicz Les Intégraphes, la courbe intégrale et ses applications, Paris 1886 verwiesen. 
beiden Wagen sind der Führungs- und Zeichenstift verbunden, die an Armen angebracht sind, die senkrecht zur Schienen (y-) Richtung stehen, also eine konstante Verschiebung der Kurven in der Richtung der Abszissen verursachen, von der wir bei der Theorie der Einfachheit wegen absehen können. Bei dem neuesten Modell läßt sich die GröBe der Basis durch Entfernung der Schienen voneinander verändern. Die beiden Stifte liegen auf verschiedenen Seiten der Schienen, weshalb das Instrument beim Gebranch mit Vorteil so aufgestellt wird, da $B$ die Schienenrichtung nach dem Beobachter zeigt, also das Instrument nach rechts oder links rollt. Die wesentliche Vereinfachung gegenüber dem Integraphen von $\mathrm{Abdank}$ Abakanowicz liegt in dem Wegfall des dritten Wagens.

Um die Wirkungsweise des Instruments zu erkennen, balten wir den Integralwagen in einer beliebigen Stellung fest und lassen den Differentialwagen laufen. Dann ändert die Direktrixschiene fortgesetzt ihre Richtung und verschiebt sich dabei in ibrem Lager auf dem Differentialwagen. Infolge dessen dreht sich die Integrierrolle auf dem Berührungspunkt mit der Zeichenebene ruhend, ohne zu rollen. LäBt man umgekehrt den Integrierwagen frei und hält man den Differentialwagen fest, bewegt dagegen das ganze Instrument in der Richtung (der $x$-Achse) senkrecht zu den Schienen, so wird die Integrierrolle in ihrer Ebene sich um ihre Achse drehen. Wenn der Winkel der Rollenebene gegen die $x$-Achse $\varphi$ ist, so wird bei einer Bewegung $d x$ des Instruments von der Rolle der Weg $\cos \varphi d x$ abgewickelt. Da die Rollenebene ihre Richtung beibehält, so findet wegen der Komponente sin $\varphi d x$ eine Bewegung des Integralwagens auf seiner Schiene, in der $y$-Richtung, statt: $d Y=d x \sec \varphi \sin \varphi=\operatorname{tg} \varphi d x$, mithin ist $\frac{d Y}{d x}=\operatorname{tg} \varphi$. Da nun die Rollenebene ibre Richtung festhält, also $\varphi$ konstant bleibt, so wird $Y=x \operatorname{tg} \varphi+C=b x+C$, d. h. der Integrierstift beschreibt eine gegen die Abszissenachse um den Winkel $\varphi$ geneigte Gerade. Wird dagegen auch der Differentialwagen losgelassen, so ändert sich fortgesetzt die Richtung der Direktrixschiene, also auch diejenige der Rollenebene, und der Integrierstift beschreibt eine stetig gekrümmte Kurve.

Wir denken uns die gegebene Differentialkurve und die Integralkurve auf dieselbe $x$-Achse bezogen. Sind $x, y$ die Koordinaten der gegebenen Kurve, $X, Y$ der Integralkurve, so ist $X-x=b$ der konstanten Basis des Apparates gleich, die wir als Einheit wählen. 
Wenn ferner $\varphi$ der Winkel der Direktrix mit der $x$-Achse, also auch der Tangente der Integralkurve ist, so bestehen die Gleichungen $\operatorname{tg} \varphi=y-Y$ und $\frac{d Y}{d x}=y-Y$. Bezeichnen wir noch $\frac{d Y}{d x}=Y^{\prime}, y=F(x)$, so erhalten wir die lineare Differentialgleichung erster Ordnung

$$
Y^{\prime}+Y=F(x),
$$

die daher durch den Apparat integriert wird.

Sind die Ordinaten beider Kurven gleich, $y=Y$, so hat die Integralkurve ein Maximum oder Minimum, weil $\frac{d Y}{d x}=0$ ist. Wenn die Tangenten entsprechender Punkte beider Kurven zusammenfallen, also $\frac{d y}{d x}=\frac{d Y}{d x}$ ist, so wird $\frac{d^{2} Y}{d x^{2}}=Y^{\prime \prime}=0$, und die Integralkurve hat eine Wendetangente parallel zur $x$-Achse. Wenn die Ordinate der Differentialkurve diskontinuierlich ist, so ist die Tangente der Integralkurve diskontinuierlich. Wenn nämlich der Fahrstift einen Kurvenbogen durchläuft und man läßt durch einen Zug den Differentialwagen auf seiner Schiene laufen, ohne den Apparat zu verrücken, und verfolgt dann mit dem Fahrstift einen andern Kurvenbogen, dessen Anfangspunkt also eine andre Ordinate, aber dieselbe Abszisse wie der Endpunkt der erst beschriebenen Kurve hat, so wechselt die Integralkurve plötzlich ihre Richtung. Sie macht, von der Unterbrechung an, einen Winkel mit der bis dahin beschriebenen Kurve, also hat die Integralkurve einen Rückkehrpunkt. Wenn dagegen die Tangente der Differentialkurve diskontinuierlich ist, aber ihre Ordinate kontinuierlich, so bleibt die Tangente der Integralkurve kontinuierlich. Wenn also der Fabrstift einen Kurvenbogen bis zu einem gewissen Punkte beschreibt, und man läBt nun plötzlich den Apparat in entgegengesetzter Richtung rollen, so entsteht bei der Integralkurve eine Spitze, da die beiden Teile der Kurve in dem betrachteten Punkte eine gemeinsame Tangente haben.

Das allgemeine Integral der Gleichung (1) ist

$$
\text { (2) } Y=e^{-x}\left[\int F(x) e^{x} d x+C\right]=Y_{0}+e^{-x} \int_{x_{0}}^{x} F(x) e^{x} d x \text {, }
$$

wenn $Y=Y_{0}$ für $x=x_{0}$ ist. 
Befährt man eine geschlossene Kurve mehrmals, z. B. einen Kreis mit der Gleichung $(x-a)^{2}+(y-b)^{2}=r^{2}$, so hat die Integralkurve in den $x=a \pm r$ entsprechenden Punkten Spitzen (vgl. S. 156) und bei mehrmaligem Durchfahren werden sowohl die Spitzen, welche $x=a+r$ entsprechen, als auch diejenigen, welche $x=a-r$ entsprechen, gleiche Ordinatendifferenzen haben. Die Ordinate vergröBert sich nach jedem vollen Umlauf um

$$
e^{-x} \int_{x_{0}}^{x_{0}} F(x) e^{x} d x,
$$

wo sich die Grenzen auf denselben Punkt ror und nach dem Umlauf beziehen. Der Faktor $e^{-x}$ ist um so kleiner, je größer die Abszisse ist, also die konstanten Ordinatendifferenzen sind für die Abszisse $x=a-r$ gröBer als für die Abszisse $x=a+r$.

Setzt man in (2) $F(x)=a$ einer Konstanten gleich, so folgt ${ }^{1}$ )

$$
Y=a+C e^{-x} \text {. }
$$

Man erbält also die Exponentialkurve. Sie nähert sich asymptotisch der Geraden $Y=a$. Wenn wir die $x$ - und $y$-Achse so legen, da $B$ der Punkt $x=0 \quad Y_{0}=a \pm 1$ ein Punkt der Kurve ist, so erhalten wir in diesem Punkte

$$
Y_{0}=a \pm 1=a+C, \text { also } C= \pm 1,
$$

mithin als Gleichung der Kurve nach (3) $Y=a \pm e^{-x}$, oder wenn wir die $x$-Achse parallel um den Abstand $a$ verschieben: $Y= \pm e^{-x}$, oder auch, wenn wir das Zeichenblatt um $180^{\circ}$ drehen, so $\mathrm{da} \bar{B}+x$ in $-x$ übergeht, $Y= \pm e^{x}$.

Verschiebt man die $y$-Achse parallel um $x=-m$, so wird $Y=e^{x+m}=M e^{x}$, und man erhält die logarithmische Kurve.

Hat man die Integralkurve $Y=e^{x}$ erhalten und durchläuft man diese Kurve als Differentialkurve mit dem Fahrstift, so erbält man eine neue Integralkurve

$$
Z=e^{-x}\left[\int e^{2 x} d x+C\right]=\frac{1}{2} e^{x}+C e^{-x} .
$$

Das Vorzeichen von $C$ hängt von der Anfangsstellung des Integralwagens ab. Beginnen wir die Durchfahrung von $F_{1}(x)=e^{x}$

1) $Y=a e^{-x} \int e^{x} d x=a e^{-x}\left(e^{x}+c\right), a c=C$. 
im Punkte $x=0, y=1$, so wird, je nachdem die Anfangsstellung des Integralwagens $Z_{0}>\frac{1}{2}, Z_{0}=\frac{1}{2}, Z_{0}<\frac{1}{2}$ gewählt wird, $C$ positiv, null oder negativ sein, da für $x=0 Z_{0}=\frac{1}{2}+C$ oder $C=Z_{0}-\frac{1}{2}$ ist.

Ist $C$ positiv, so stellt (4) eine Kettenlinie dar. Wenn nämlich der Anfangspunkt auf der $x$-Achse verschoben wird, so da $B$ $x=x^{\prime}+m$ ist, so erhält man mit $m=\frac{1}{2} \log 2 C$ die Gleichung in der Form $Z=\frac{1}{2} e^{m}\left(e^{x^{\prime}}+e^{-x^{\prime}}\right)$, also die Kettenlinie mit dem Parameter $e^{m}=\sqrt{2} C$. Ist $C=\frac{1}{2}\left(Z_{0}=1\right)$, so erhält man die Kurve $Z=\frac{1}{2}\left(e^{x}+e^{-x}\right)=\cos i x=$ (Eo $x$. Ist $C=0\left(Z_{0}=\frac{1}{2}\right)$, so wird $Z=\frac{1}{2} e^{x}$, also ist die Integralkurve wiederum eine Exponentialkurve. Ist endlich $C$ negativ, so erhält man

$Z=\frac{1}{2} e^{m}\left(e^{x}-e^{-x}\right)$ und für $m=0 \quad Z=\frac{1}{2}\left(e^{x}-e^{-x}\right)=$ Sin $x$.

Auch mit diesem Apparat lassen sich algebraische Gleichungen lösen. Setzt man in (2)

$$
F(x)=e^{-x}, \text { so ist } \quad Y_{1}=e^{-x}(x+C) .
$$

Integriert man diese Kurve nochmals, so liefert der Apparat ${ }^{1}$ )

und so fort. ${ }^{2}$ )

$$
Y_{2}=e^{-x}\left(\frac{1}{2} x^{2}+C x+C^{\prime}\right)
$$

Wird der Schienenabstand verstellbar eingerichtet, so daB man statt der Basis 1 die Basis $\frac{1}{a}$ erhält, so ist wenn

1) $Y_{2}=e^{-x}\left[\int F(x) e^{x} d x+C^{\prime}\right]=e^{-x}\left[\int(x+C) d x+C^{\prime}\right]$,

eingesetzt wird.

$$
F(x)=e^{-x}(x+C)
$$

2) Beispiel:

$$
\begin{aligned}
& \frac{1}{6} x^{3}+\frac{1}{2} M x^{2}+N x+P=0 \\
& \frac{1}{2} x^{2}+M x+N=0 \\
& x+M=0
\end{aligned}
$$

1. Differentialkurve: $y=e^{-x}$. Einstellung des Differentialwagens:

$$
x_{0}=0, y_{0}=1
$$

Integralkurve: $\quad Y_{1}=e^{-x}(x+C)$. Einstellung des Integralwagens: $x_{0}=0, Y_{0}=M$, also

2.

$$
\begin{aligned}
& Y_{1}=e^{-x}(x+M) \quad x_{0}=0, y_{0}=M \\
& Y_{2}=e^{-x}\left(\frac{x^{2}}{2}+M x\right)+e^{-x} C \quad x_{0}=0, Y_{0}=N
\end{aligned}
$$




$$
\operatorname{tg} \varphi=a(y-Y) \text { und } \frac{d Y}{d x}=a y-a Y,
$$

also mit

$$
\frac{d Y}{d x}=Y^{\prime \prime}, \quad Y^{\prime}+a Y=a y .
$$

Integriert man die hurve $Y$ ron neuem und wählt die Basis $\frac{1}{b}$, so erhält man

$$
Z^{\prime}+b Z=b Y=b y-\frac{b}{a} Y^{\prime}
$$

oder

$$
a Z^{\prime}+a b Z-a b y+b Y^{\prime}=0 .
$$

Nun erhält man durch Differentiation $Z^{\prime \prime}+b Z^{\prime}-b Y^{\prime}=0$, oder wenn man den Wert für $b Y^{\prime}$ einsetzt,

$$
Z^{\prime \prime}+(a+b) Z^{\prime}+a b Z=a b y,
$$

wo $y=f(x)$ die ursprünglich gegebene Kurre ist. Man bann in dieser Weise fortfahren und erhält Differentialgleichungen ron beliebiger Ordnung mit konstanten Koeffizienten. Der Apparat zeichnet die Kurve der Funktion $Z$, welche der Differentialgleichung genügt.

Sollen die Koeffizienten nicht Konstante, sondern Funktionen von $x$ sein, so muB die Basis eine Funktion ron $x$ sein, also durch einen Mechanismus der Schienenabstand stetig geändert werden. Dieser Mechanismus müBte mit einem Stifte verbunden sein, mit dem man eine gegebene Kurve $\Phi(x)$ umfährt, aber die gleichzeitige Führung ron zwei Stiften auf zwei Kurven hätte Schwierigkeiten, und es müBte ein zweiter Beobachter tätig sein. ${ }^{1}$ )

3.

$$
\begin{array}{ll}
Y_{2}=e^{-x}\left(\frac{x^{2}}{2}+M x+N\right) & x_{0}=0, y_{0}=N \\
Y_{3}=e^{-x}\left(\frac{x^{3}}{6}+\frac{1}{2} M x^{2}+N x+C\right) & x_{0}=0, Y_{0}=P \\
Y_{3}=e^{-x}\left(\frac{x^{3}}{6}+\frac{1}{2} M x^{2}+N x+P\right) .
\end{array}
$$

Die Schnittpunkte der Kurve $\boldsymbol{Y}_{3}$ mit der $x$-Achse geben die reellen Wurzeln der vorgelegten Gleichung.

1) Wenn $a$ eine Funktion ron $x$ ist, stellt die Gleichung $Y^{\prime}+a Y^{\prime}$ $=a y$ die Form dar, auf die eive lineare Differentialgleichung erster Ordnung stets gebracht werden kann. 
Wesentlich einfacher ist eine andere Abänderung (Fig. 86), welche der Ebene der Rolle eine beliebige konstante Neigung $\alpha$ gegen

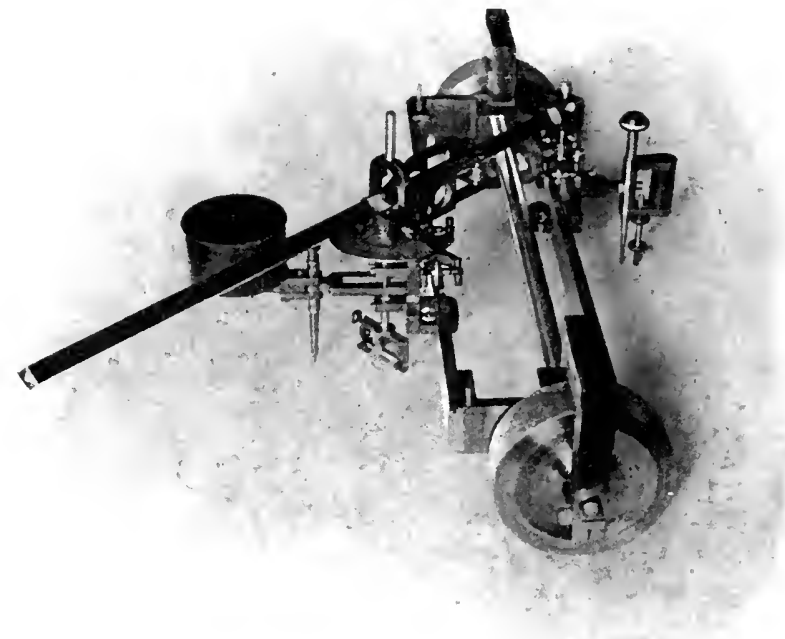

Fig. 86.

die Direktrixschiene gibt. Sei $\alpha$ der Winkel, so daB der Winkel der Rollenebene gegen die $x$-Achse $\varphi+\alpha$ wird, so erhält man

$$
\begin{gathered}
\frac{d Y}{d x}=\operatorname{tg}(\varphi+\alpha)=\frac{\operatorname{tg} \varphi+\operatorname{tg} \alpha}{1-\operatorname{tg} \varphi \operatorname{tg} \alpha}, \text { oder mit } \operatorname{tg} \alpha=m \\
y-Y=\operatorname{tg} \varphi=\frac{Y^{\prime}-m}{1+m Y^{\prime}},
\end{gathered}
$$

oder

oder

$$
Y=\frac{m-Y^{\prime}}{1+m Y^{\prime}}+y,
$$

$$
Y^{\prime}[1+m(Y-y)]+Y-y-m=0 .
$$

Dies ist also eine Differentialgleichung, die der umgeänderte Apparat integriert.

Wird im Besonderen die Rolle senkrecht zur Richtung der Direktrix gestellt, also der Winkel $\alpha=90^{\circ}$ gemacht, so integriert 
das Instrument die Differentialgleichung

$$
Y=\frac{1}{Y^{\prime}}+y=\frac{1}{Y^{\prime}}+f(x) .
$$

Diese Vorrichtung, welche die Rollenebene um eine vertikale Achse beliebig zu drehen gestattet, kann auch bei dem Instrumente von $\mathrm{Abdank-Abakanowicz} \mathrm{angebracht} \mathrm{werden.} \mathrm{Der} \mathrm{Drehungs-}$ winkel $\alpha$ wird dabei an einem horizontalen, geteilten Kreise eingestellt. Wir werden die Instrumente von $\mathrm{Abdank}$ und Pascal kurz durch $A$ und $P$ bezeichnen. Da der Unterschied in der Theorie darin liegt, daB bei $P: y-Y=a \operatorname{tg} \varphi$, bei $A: y=a \operatorname{tg} \varphi$ ist, wenn $a$ die (gewöhnlich $=1$ gesetzte) Einheitsstrecke oder Basis des betreffenden Instrumentes bedeutet, so erhalten wir bei $A$ einfacher mit $\operatorname{tg} \alpha=m$

$$
Y^{\prime}=\frac{a m+f(x)}{a-m f(x)} .
$$

Für $\alpha=-90^{\circ}$ und $a=1$ wird ferner bei $A$

$$
Y^{\prime}=\frac{1}{f(x)} \text { oder } Y=\int \frac{d x}{f(x)}+C .
$$

Wählt man im Besondern $f(x)=x$ d. h. als Differentialkurve eine um $45^{\circ}$ gegen die Abszissenachse geneigte Gerade, so zeichnet der Integrierstift für gegebene Grenzen die Kurve

$$
Y=\log x .
$$

Läßt man nun die Stellung der Rolle $\left(\alpha=-90^{\circ}\right)$ unverändert und verwendet man die soeben erhaltene logarithmische Kurve als Differentialkurve, so beschreibt der Integrierstift, indem man $f(x)=\log x$ einzusetzen hat, die Funktion

$$
Y=\int \frac{d x}{\log x},
$$

die zuerst von Euler ${ }^{1}$ ) betrachtet und von Soldner ${ }^{2}$ ) Integrallogarithmus benannt worden ist. $G a u B^{3}$ ) hat darauf aufmerk-

1) Institutiones calculi integralis I, Kap. IV.

2) Théorie et tables d'une nouvelle fonction transcendente. München 1809. Vgl. L. Kronecker, Vorlesungen über die Theorie der einfachen and vielfachen Integrale, herausg. v. E. Nett o, Leipzig(Teubner) 1894, $199-214$.

3) Werke II, 444. (Brief an Encke.) 
sam gemacht, daB dieses Integral die Anzahl aller Primzahlen unter einer gegebenen Grenze nahezu darstellt. Das Instrument gibt daher eine zahlentheoretische Anwendung, indem die Ordinate $Y$ die Anzahl der Primzahlen unter der durch die zugehörige Abszisse $x$ dargestellten Zahl miBt.

Noch zwei andere Abänderungen des Integraphen rühren von Pascal her.

Die erste besteht darin, $d a B$ an Stelle der einen geradlinigen Schiene, nämlich derjenigen, auf welcher der Integralwagen läuft, eine gekrümmte Schiene eingesetzt wird, welche die Gestalt einer vorgeschriebenen Kurve besitzt.

Denken wir uns durch den Drehpunkt der Direktrix am Differentialwagen eine Parallele zur $y$-Achse gelegt, die also nahe mit der Differentialschiene zusammenf ällt, und den Anfangspunkt des mit dem Instrumente beweglichen Koordinatensystems auf der festen $x$-Achse, so kann man sich die Gleichung der Schienenkurve in der Form $\xi=\Phi(Y)$ gegeben denken. Dies ist zugleich die Kurve, welche der Integrierstift bei ruhendem Instrument beschreibt, wenn sein Wagen auf der gekrümmten Schiene läuft, nur um ein konstantes Stück in der Abszissenrichtung verschoben. Es ist nun $X=\xi+x$ und daher $y=f(X-\xi)=f(X-\Phi(Y))$. Wird noch die Rollenebene drehbar angenommen, so erhalten wir bei Instrument $A$ mit $m=\operatorname{tg} \alpha$

$$
Y^{\prime}=\frac{m a+f\left(X-\Phi\left(Y^{\prime}\right)\right)}{a-m f(X-\Phi(Y))} .
$$

Beim Instrument $P$ ist $a$ der Abszissenunterschied des Integrierstiftes und Drehpunktes der Direktrix, mithin tritt bei dem abgeänderten Instrument $\xi$ an Stelle von $a$ und aus $Y^{\prime}=\operatorname{tg}(\varphi+\alpha)$ und $y-Y=\xi \operatorname{tg} \varphi$ folgt

$$
Y^{\prime}=\frac{m \Phi(\boldsymbol{Y})+f(X-\Phi(\boldsymbol{Y}))-\boldsymbol{Y}}{\Phi(\boldsymbol{Y})-m[f(\boldsymbol{X}-\Phi(\boldsymbol{Y}))-Y]} .
$$

Die zweite $A b a ̈ n d e r u n g$, die ohne Weiteres nur beim Instrument $P$ angebracht werden kann, läBt die geradlinigen Schienen unverändert, ersetzt dagegen die Direktrix durch eine Kurve von gegebener Form. Die Integrierrolle wird dabei so gefübrt, daB ihre Ebene stets die Tangente dieser Kurve enthält. $\left.{ }^{1}\right)$ Der ver-

1) In einer während des Druckes erschienenen Abhandlung: Sul mio integrafo a riga curvilinea, Napoli 1912, gibt Pascal eine ver- 
änderliche Winkel, den dann die Rollenebene mit der geraden Linie bildet, welche die Direktrix in ihrer ursprünglicben Gestalt darstellt, sei $\alpha$ und $\varphi+\alpha$ der Winkel mit der $x$-Achse. Der Winkel $\alpha$ hängt von der Stellung der beiden Wagen auf ihren Schienen bei ruhendem Instrument, also von $y-Y$ ab und wir können daher $\operatorname{tg} \alpha=\psi(y-Y)$ setzen. Wegen $Y^{\prime}=\operatorname{tg}(\varphi+\alpha)$, $y-Y=a \operatorname{tg} \varphi, y=f(x)$ wird dáher

$$
Y^{\prime}=\frac{f(x)-Y+a \psi(f(x)-Y)}{a-[f(x)-Y] \psi(f(x)-Y)}=\Psi(f(x)-Y) .
$$

Auch die gleichzeitige Anbringung beider Variationen bat Pascal untersucht.

Polarintegraph. Wir wenden uns noch einer andern Form eines Integraphen zu, den Pascal konstruiert hat. Ein Kreisquadrant ruht 1.) drehbar in seinem Mittelpunkte fest auf der Zeichenebene, 2.) auf einer am Ende des einen begrenzenden Radius angebrachten Walze und 3.) auf der an einem beweglichen Radius verschiebbaren Integrierrolle, also auf drei Punkten auf und kann auf der Walze rollend um den Mittelpunkt rotieren. Der fest mit dem Kreisbogen verbundene, zuerst genannte Radius, an dem die Walze sitzt, dient als Schiene für einen Differentialwagen, während der Integralwagen auf dem gegen diesen Radius um einen (am Kreise ablesbaren) Winkel verstellbaren andern Schienenradius sich bewegt. Beide Wagen sind durch eine geradlinige Direktrixstange verbunden, die sich um Zapfen an beiden Wagen drehen und auBerdem in ihrer Längsrichtung verschieben kann. Zu letzterem Zwecke ist sie über ein Lager geführt, das der Zapfen des Differentialwagens trägt. Wir sehen für die Theorie des Instrumentes davon $a b, d a B$ der Zeichenstift des Integralwagens aus praktischen Gründen seitlich, aber in derselben Entfernung vom Mittelpunkt, den wir auch als Pol bezeichnen wollen, angebracht ist. Wir nehmen vielmehr an, daB der Berührungspunkt der am Integralwagen befindlichen Rolle, deren Ebene mit der Richtung der Direktrix in jedem Augenblicke zusammenfällt, die Integralkurre beschreibt.

In der Anfangsstellung des Instruments möge der die Differentialschiene bildende Radius mit einer in der Zeichenebene gewählten

besserte Konstruktion an, bei der die gekrümmte Direktrix-Schiene sich um einen Zapfen des Integralwagens dreht wäbrend der Zapfen des Differentialwagens sich längs der Schienenkurve verschiebt. Die Rollenebene ist dabei fest mit der Schiene verbunden. 
Anfangsrichtung den Winkel $\vartheta$ bilden. Ist $\varrho$ die der Stellun des Wagens entsprechende Entfernung des Differentialstiftes von Pole, so sei $\varrho=f(\vartheta)$ die Gleichung der gegebenen Kurve. Des am Kreisbogen abgelesene Winkel der beiden Schienen sei $\omega$, der Leitstrahl des Berührungspunktes der Integrierrolle, $90^{\circ}-9$ der Winkel ihrer Ebene oder der Verlängerung der Direktrix mi dem Radius $r$, so ist

$$
\operatorname{tg} \varphi=\frac{d r}{r d} \frac{\left.d_{1} r+\omega\right)}{(\boldsymbol{v}+\omega}=\frac{d r}{r d \boldsymbol{v}},
$$

da $\omega$ während der Ausführung der Integration ungeändert bleibt Die Länge der Direktrix (zwischen ihren Drehpunkten auf beider Wagen) ist

$$
\delta=\sqrt{\varrho^{2}+r^{2}-2 \varrho r \cos \omega} .
$$

Aus dem Dreieck mit den Seiten $\varrho, r$ und $\delta$ folgt

mithin

$$
\cos \varphi=\frac{\rho \sin \omega}{\sqrt{r^{2}+\varrho^{2}-2 r \rho \cos \omega}} \text { und } \operatorname{tg} \varphi=\frac{r-\rho \cos \omega}{\rho \sin \omega}
$$

oder

$$
\frac{d r}{d \vartheta}=\frac{r^{2}}{\sin \omega f(\boldsymbol{\vartheta})}-r \operatorname{ctg} \omega
$$

$$
\frac{d\left(\frac{1}{r}\right)}{d \vartheta}-\frac{1}{r} \operatorname{ctg} \omega=-\frac{1}{\sin \omega \cdot f(\vartheta)} .
$$

In diese lineare Form kann eine Bernoullische Gleichun $\frac{d y}{d x}+P y=Q y^{n}$ gebracht werden, wenn $P$ konstant ist.

57. Integratoren für Differentialgleichungen. Die Inte graphen von Pascal können zwar Quadraturen ausführen, sin aber in erster Linic für die Integration von Differentialgleichunge bestimmt. Zu letzterem Zwecke sind noch verschiedene Instrument, konstruiert worden: von Lord Kelvin 1876, Torrès 1895 Petroviteh $1897^{1}$ ). Wir fügen noch eine kurze Beschreibun von zwei Apparaten von L. Jacob, Generalingenieur der franzö sischen Schiffsartillerie, hinzu, welche zwei besondere Differential gleichungen integrieren. ${ }^{2}$ ) Sie sind in ihrem Hauptteil einem Prytz

1) Vgl. L. Jacob, Le calcul mécanique, wo sich die Literatur an gegeben findet.

2) In einer soeben erschienenen Abhandlung: C. Ajello, Su di un importante applicazione dell' integrafo $\mathrm{Pascal}$ a riga curvilinea, Napol 1912, wird gezeigt, daB auch der Pa s cal sche Integraph mit gekrümm ter Direktrix die Riccatische und Abelsche Gleichung integriert 
schen Stangenplanimeter ähnlich, bei dem die Schneide durch ein scharfrandiges Rad ersetzt ist. (S. S. 122).

Der Fahrstift ist bei dem ersten zur Auflösung der Riccati schen Gleichurg bestimmten Apparate durch den Mittelpunkt eines geteilten Kreises hindurchgeführt. Ein Durchmesser dieses horizontalen Kreises wird durch zwei miteinander verbundene Gelenkparallelogramme stets einer bestimmten gegebenen Richtung parallel gehalten, wenn der Fahrstift auf der gegebenen Kurve entlang geführt wird. Dieser ganze Mechanismus wird von kleinen Rollen getragen, damit der Kreis und die Parallelogramme mit möglichst wenig Reibung sich bewegen können. An der Stange des Apparates ist ein Nonius angebracht, der an dem Kreise gleitet und den Winkel, den die Stange in irgend einem Augenblick mit der Anfangsrichtung einschlieBt, abzulesen gestattet.

Wie beim Stangenplanimeter ist die Richtung der Stange jederzeit Tangente an die rom Berührungspunkte des Schneidenrades beschriebene Kurre. Es seien $X, Y$ die Koordinaten dieses Berührungspunktes, $x, y$ diejenigen des Fahrstiftes; $\omega$ sei der Winkel, den die Stange mit der Abszissenachse, die wir als Anfangsrichtung wählen, einschlieBt, o die Länge der Stange, d. h. der Abstand rom Fahrstift bis zum Mittelpunkt des Schneidenrades. Es ist dann

$$
\frac{d Y}{d X}=\operatorname{tg} \omega
$$

ferner

(2)

$$
X=x+\varrho \cos \omega, Y=y+\varrho \sin \omega
$$$$
d X=d x+\cos \omega d \underline{-}-\underline{\sin \omega} \omega \mathrm{d \omega}
$$$$
d Y=d y+\sin \omega d \rho+\varrho \cos \omega d \omega,
$$

woraus durch Jultiplikation der ersten Gleichung mit - sin $\omega$, der zweiten mit $+\cos \omega$ und Addition mit Rücksicht auf (1) folgt:

$0=-\sin \omega d X+\cos \omega d Y=\rho d \omega+\cos \omega d y-\sin \omega d x:$

Wir führen eine unabhängige Variable $t$ ein und denken uns die gegebene, vom Fahrstift beschriebene Kurre durch

definiert. Es ist dann

$$
x=f_{1}(t), y=f_{2}(t)
$$

$$
d x=f_{1}^{\prime}(t) d t, d y=f_{9}^{\prime}(t) d t
$$


und nach (4)

$$
\varrho \frac{d \omega}{d t}+f_{2}^{\prime}(t) \cos \omega-f_{1}^{\prime}(t) \sin \omega=0 .
$$

Führt man noch $\operatorname{tg} \frac{1}{2} \omega=u$ ein, dann ist

$$
d u=\frac{\frac{1}{2} d \omega}{1+u^{2}}, \frac{d \omega}{d t}=\frac{2 \frac{d u}{d t}}{1+u^{2}}, \cos \omega=\frac{1-u^{2}}{1+u^{2}}, \sin \omega=\frac{2 u}{1+u^{2}},
$$

und man erhält nach (5) für die Winkelbewegung der Stange die Differentialgleichung

$$
2 \varrho \frac{d u}{d t}+\left(1-u^{2}\right) f_{2}^{\prime}(t)-2 u f_{1}^{\prime}(t)=0
$$

oder für eine bestimmte Stangenlänge $\varrho=l$ (die Stangenlänge läßt sich bei dem Apparate ändern)

$$
\frac{d u}{d t}=u^{2} \frac{f_{2}^{\prime}(t)}{2 l}+u \frac{f_{1}^{\prime}(t)}{l}-\frac{f_{2}^{\prime}(t)}{2 l} .
$$

Man nennt nun eine allgemeine Riccatische Gleichung eine Differentialgleichung von der Form:

$$
\frac{d u}{d t}=P u^{2}+Q u+R
$$

wo $P, Q, R$ Funktionen von $t$ sind. Im vorliegenden Falle besteht aber die einschränkende Bedingung $P+R=0$. Soll diese Gleichung integriert werden, so hat man (vgl. (7) und (8))

$$
\frac{d y}{d t}=f_{2}^{\prime}(t)=2 l P=-2 l R, \frac{d x}{d t}=f_{1}^{\prime}(t)=l Q
$$

also

$$
y-y_{0}=2 l \int P d t, x-x_{0}=l \int Q d t
$$

zu setzen, wo $y_{0}$ und $x_{0}$ Integrationskonstante sind oder auch die Koordinaten, die dem Werte $t_{0}$ entsprechen, bedeuten. Durch $x$ und $y$ ist dann die vom Fahrstift zu durchlaufende Kurve gegeben.

Das Integral bis zur Grenze $t=t_{1}$ erhält man dann, indem man den Winkel $\omega_{1}$ am Instrument abliest, nämlich $u_{1}=\operatorname{tg} \frac{\omega_{1}}{2}$.

Der allgemeine Fall, in dem $P+R \gtrless 0$ ist, läBt sich auf den besondern zurückführen. Setzt man $u=\lambda v$, wenn $\lambda$ eine noch unbestimmte Funktion von $t$ ist, so hat man aus (8) 


$$
u^{\prime}=\lambda v^{\prime}+v \lambda^{\prime}=P \lambda^{2} v^{2}+Q \lambda v+R
$$

also

$$
v^{\prime}=P \lambda v^{2}+\left(Q-\frac{\lambda^{\prime}}{\lambda}\right) v+\frac{R}{\lambda} .
$$

Bestimmt man nun $\lambda$ so, daB

$$
P \lambda+\frac{R}{\lambda}=0 \text { oder } \lambda= \pm \sqrt{-\frac{R}{P}}
$$

ist, so ist der allgemeine auf den speziellen Fall zurückgeführt. Jedoch erbält man nur dann eine reelle Wurzel $\lambda$, wenn $R$ und $P$ verschiedene Vorzeichen haben.

Wenn dies in dem in Betracht kommenden Intervall von $t_{0}$ bis $t_{1}$ nicht der Fall ist, führt man den als bekannt anzunehmenden Winkel $\beta$ der Tangente in einem beliebigen Punkte der gegebenen Kurve mit der $x$-Acbse ein. Dann bilden die Tangenten in den entsprechenden Punkten beider Kurven miteinander den Winkel $\beta-\omega=\vartheta$.

$\mathrm{Da}$

$$
\frac{d y}{d x}=\operatorname{tg} \beta \text { und } l d \omega+\cos \omega d y-\sin \omega d x=0
$$

ist (s. (4)), so folgt

$$
l d \beta-l d \vartheta+\cos (\beta-\vartheta) d y-\sin (\beta-\vartheta) d x=0
$$

oder auch wegen $\cos \beta d y-\sin \beta d x=0$

$$
l d \vartheta=l d \beta+\sin \beta \sin \vartheta d y+\cos \beta \sin \vartheta d x .
$$

Setzt man nun

$$
v=\operatorname{tg} \frac{1}{2} \vartheta, d \vartheta=\frac{2 d v}{1+v^{2}}, \sin \vartheta=\frac{2 v}{1+v^{2}},
$$

so erhält man, wenn die Ableitungen nach $t$ genommen werden, durch Einsetzen

$$
\frac{d v}{d t}=v^{2} \frac{1}{2} \frac{d \beta}{d t}+v \frac{1}{l}\left[\sin \beta \frac{d y}{d t}+\cos \beta \frac{d x}{d t}\right]+\frac{1}{2} \frac{d \beta}{d t},
$$

also eine Gleichung von der früheren Form (10), in $\operatorname{der} P \lambda=\frac{R}{\lambda}$ ist und $\lambda=\sqrt{\frac{R}{P}}$ wird.

Wenn also

$$
P=\frac{1}{2} \frac{d \beta}{d t}, Q=\frac{1}{l}\left[\sin \beta \frac{d y}{d t}+\cos \beta \frac{d x}{d t}\right]
$$


gesetzt wird, so folgt

$\beta-\beta_{0}=2 \int_{t_{0}}^{t_{1}} P d t, x-x_{0}=l \int_{i_{0}}^{t_{1}} Q \cos \beta d t, y-y_{0}=l \int_{t_{0}}^{t_{1}} Q \sin \beta d t$.

Für $t=t_{0}$ kennt man $x_{0}, y_{0}, \beta_{0}$, ferner ist $\omega_{0}=\beta_{0}-\vartheta_{0}$ gegeben; für $t=t_{1}$ erhält man die gesuchte Lösung

$$
v_{1}=\operatorname{tg} \frac{1}{2} \vartheta_{1}=\operatorname{tg} \frac{1}{2}\left(\beta_{1}-\omega_{1}\right) \text {. }
$$

Der $\mathrm{z}$ weite Apparat von Jacob dient zur Integration der Abelschen Gleichung

$$
y^{\prime}=p y^{3}+q y^{\circ}+r y+s,
$$

wo $p, q, r, s$ Funktionen von $x$ sind und, $y^{\prime}=\frac{d y}{d x}$ ist. Durch die Substitution

$$
y=\frac{1}{u}, y^{\prime}=-\frac{u^{\prime}}{u^{2}}
$$

gelangt man zu der Form

$$
-u u^{\prime}=p+q u+r u^{2}+s u^{3} .
$$

Zunächst wird $s=0$ angenommen und wir schreiben

$$
u u^{\prime}=P u^{2}+Q u+R \text {. }
$$

Das Instrument ist ähulich dem vorigen konstruiert. Wir denken uns wiederum ein Stangenplanimeter, dessen Fahrstift aber im Anfangspunkt einer horizontal liegenden Kurve $C$ angebracht ist, die als eine gekrümmte Schiene einen Bestandteil des Instrumentes darstellt. Der Fabrstift führt bei seiner Bewegung längs einer gegebenen Kurve diese Schiene mit sich. Durch eine Parallelführung ist in derselben Weise wie beim vorigen Instrument dafür gesorgt, da $B$ diese Kurvenschiene keine drehende Bewegung erfährt, sondern eine bestimmte Tangente der Kurve $C$ bei jeder Stellung des Fahrstiftes einer auf der Zeichenebene gegebenen Anfangsrichtung parallel bleibt. Die Verschiebungen der Gelenkparallelogramme und der Kurvenschiene erfolgen nicht durch Gleiten auf der Papierebene, sondern auf kleinen Rollen.

Auf der Stange des Instruments ist nun eine Hülse verschiebbar angebracht, die unterhalb mit vertikalen und horizontalen Rädchen in der im Querschnitt U-förmig aufgebogenen Kurvenschiene geführt wird. Oben enthält diese Hülse ein Fenster, durch das man die Entfernung $\rho$ des darunter befindlichen Kurvenpunktes 
vom Anfangspunkt der Kurve $C$ ablesen kann. Ebenso kann man auch an der passend geteilten Kurrenschiene den Winkel $\omega$ mit der Anfangsrichtung ablesen.

Es seien $x, y$ die Koordinaten eines Punktes der Kurve, die der Fabrstift durchläuft, und $t$ eine unabhängige Variable. Dann ist wie oben, wenn die Akzente die Ableitungen nach $t$ bezeichnen,

$$
\rho \frac{d \omega}{d t}=x^{\prime} \sin \omega-y^{\prime} \cos \omega
$$

oder mit $\operatorname{tg} \frac{1}{2} \omega=u$ :

$$
2 \stackrel{v}{u^{\prime}}=u^{2} y^{\prime}+2 u x^{\prime}-y^{\prime} \text {. }
$$

$\varrho$ sehen wir als eine Funktion ron $\omega$ oder ron $u$ an. Um den Apparat auf die Abelsche Gleichung anwenden zu können, müssen wir $\varrho=l \cdot u$ setzen, wo $l$ eine zunächst noch unbestimmte Länge ist. Die Kurvenform der Schiene ist dann durch

$$
\varrho=f(\omega)=l \operatorname{tg} \frac{\omega}{2}
$$

bestimmt. Entspricht dem Werte $t=t_{0}$ die Entfernung $\varphi_{0}=l \imath_{0}$, wo $u_{0}=\operatorname{tg} \frac{\omega_{0}}{2}$ als gegeben anzusehen ist, so ist damit $l$ als Grundlänge oder Basis des Instruments bestimmt.

Die Kurve, auf der der Fahrstift geleitet wird, folgt aus

$$
y=y_{0}+2 l \int P d t, \quad x=x_{0}+l \int^{2} Q d t .
$$

Für einen andern Wert $t=t_{1}$ liest man den Wert $\rho_{1}$ am Instrument $\mathrm{ab}$ und erhält

$$
u_{1}=\frac{\rho_{1}}{l}
$$

als Integral der Differentialgleichung zwischen den Grenzen $t_{0}$ und $t_{1}$.

Die integrierte Gleichung ist aber nur ein besonderer Fall der Abelschen Gleichung, nämlich derjenige, wo $P+R=0$ ist. Um die allgemeine Gleichung hierauf zurückzufübren, setzen wir $u=\lambda z$, erhalten

$$
z z^{\prime}=z^{2}\left[P-\begin{array}{l}
\lambda^{\prime} \\
\lambda
\end{array}\right]+\frac{Q}{i} z+\frac{R}{\lambda^{2}}
$$

und bestimmen die Funktion $\lambda$ von $t$ so, daB $\lambda \lambda^{\prime}-P \lambda^{2}=R$ wird.

Diese Gleichung können wir auch folgendermaßen schreiben:

$$
\frac{d\left(\lambda^{2}\right)}{d t}-2 P \lambda^{2}=2 R,
$$

Galle: Mathematische Instrumente. 
und es folgt

$$
\lambda=e^{+\int P d t} \cdot \sqrt{\gamma+2 \int R e^{\int-2 P d t} d t} .
$$

Durch passende Wahl der Integrationskonstanten $\gamma$ wird man es in der Regel erreichen können, daß in dem in Betracht kommenden Intervall $\left(t_{0}\right.$ bis $\left.t_{1}\right) \lambda$ reell ist. Sonst muß man dem besondern Falle entsprechend eine andere Behandlung der Aufgabe versuchen.

Ist der Koeffizient $s$ von $u^{3}$ in der letzten Form der Abelschen Gleichung nicht null, so wird der Apparat komplizierter, indem an Stelle der Parallelführung eine Führung der Kurvenschiene tritt, die durch die Koeffizienten der Gleichung vorgeschrieben ist. 


\section{Literatur.}

Br. Abdank-Abakanowicz, Les Intégraphes, la courbe intégrale et ses applications. Paris $\mathbf{1 8 8 6 .}$

A. Adler, Fünfstellige Logarithmen. Leipzig 1909. [Sammlung Göschen].

Amsler, Momentenplanimeter Vierteljahrsschrift der naturforschenden Gesellschaft. Zürich, Bd. 1, 1856.

J. A msler-Laffon, Neuere Planimeter-Konstruktionen. Zeitschr. f. Instrumentenk. 4, 11. 1884.

A. Amsler, Das Planimeter und seine Erfindung. Zeitschr. des Vereins Schweizer Konkordatsgeometer 5, 117, 125. 1907.

Ch. Babbage, Passages from the life of a philosopher. London 1864.

H. P. Babbage, Babbages Analytical Engine. Monthly Notices 70, 517. 1910.

F. W. BarfuB, Handbuch der Feld-MIeBkunde. 4. Aufl. ron W. Jcep. Weimar 1889.

G. r. Bezold, Wissenschaftliche Instrumente im Germanischen Museum. Bd. I. Nürnberg 1899.

G. Coradi, Der harmonische Analysator mit einer Theorie desselben ron Prof. O. Henrici in London Zürich 1594.

-, Präzisionspolarplanimeter (Hohmann u Coradi D. R. P. 1237i), Zeitschr. f. Vermessungsw. 10, 127. 1881.

-, Die Planimeter Coradi. 3. Aufl. Zürich 1905.

C. Culmann, Die graphische Statik. 2. Anfl. Bd. 1. Zürich 1875.

E. Doležal, Planimeterstudien. Berg- und hüttenmännisches Jabrbuch der k. k. montanistischen Hochschulen zu Leoben und Přibram. 54, 293. Wien 1906. [Enthält auch ausführliche Literaturangaben].

Doll, Untersuchung der Genauigkeit vol Planimetern. Zeitschr. f. Vermessungsw. 9, 28.1880.

W. v. Dücker, Eine Aufgabe aus der Kinematik. Archiv f. Mathematik und Physik (3) 8, 151. 1908.

W. Dyck, Katalog mathematischer und mathematisch-physikalischer Modelle, Apparate und Instrumentc. München. 1892;93.

A. Emch, Kinematische Gelenksysteme. Jahresbericht der Kantonschule Solotburn. 1906/07.

Encyclopaedia Britannica. Vol. 6 (30 of complete work), 575. 1902.

Ad. Ernst, James Watt und die Grundlagen des modernen Dampfmaschinenluaes. Berlin 1897. (Vortrag auf der 37. Hauptrersammlung des Vereins deutscher Ingenieure zu Stuttgart.)

B. K. Esmarch, Die Kunst des Stabrechnens. Leipzig 1896.

A. Favaro, Sulla elica calcolatoria di Fuller. Atti del R. Istituto Veneto di scienze, lettere ed arti (5) 5, 495. Venezia 187 is. [Enthält Gieschichte und Klassifikation der Rechenschieber.] 
Fenner, Beitrag zur Theorie des Rollplanimeters. Zeitschr. f. Vermessungsw. 15, 216.1886.

S. Finsterwalder, Harmonische Analyse mittels des Polarplanimeters. Zeitschr. f. Math. u. Physik 43, 85. 1898.

H. Fürle, Zur Theorie der Rechenschieber. Jahresbericht der 9. Realschule. Berlin 1899. Progr. Nr. 126.

A. Goering, Anleitung zum Gebrauch des Rechenstabes. 3. Aufl. Berlin 1904. (Gebr. Wi chmann.)

Grabowski, Theorie des harmonischen Analysators. Sitzungsber. der k. Akad. d. Wissensch. math. naturw. Kl. Wien. 110, $717-890,1903$.

F. Günther, Äquidistanzplanimeter. Zeitschr. f. Vermessungsw. 11, 353. 1882.

-, Versuche ïber die Genauigkeit des Äquidistanzplanimeters. Zeitschrift f. Vermessungsw. 12. 1883.

-, Meßplanimeter für schmale, langgestreckte Figuren. Zeitschr. f. Vermessungsw. 15, 506. 1886.

J. Hamann, Über das Stangenplanimeter, insbesondere ein Stangenplanimeter mit Rolle. Zeitschr. f. Vermessungsw. 25, 643, 1896.

-, Das Koordinatenplanimeter. Zeitschr f. Vermessungsw. 28, 464. 1899.

- Untersuchungen über das Harfenplanimeter von Mönkemöller. Zeitschr. f. Vermessungsw. 28, 549. 1899 (vgl. 24, 331. 1895).

E. Hammer, Der logarithmische Rechenstab und sein Gebrauch. 4. Aufl. Stuttgart 1908. [Eine der vollständigsten Anleitungen mit historischen Notizen.]

-, Auflösung quadratischer Gleichungen mit dem Rechenschieber. [Verfahren von Engeler.] Zeitschr. f. Vermessungsw. 19, 495. 1900 .

-, Das Stangenplanimeter von Prytz nebst einzelnen Bemerkungen zur Praxis des Polarplanimeters. Zeitschr. f. Instrumentenk. 15, 90.1895 .

-, Referate in der Zeitschrift für Instrumentenkunde.

IIartmann, Referate in der Zeitschrift für Instrumentenkunde.

F. R. Helmert, Die Ausgleichungsrechnung nach der Methode der kleinsten Quadrate. 2. Aufl. Leipzig u. Berlin 1907. B. G. Teubner. (S. 3 Theorie des Polarplanimeters.)

O. Henrici, On a new harmonic analyser. Philosophical Magazine. (5) $38,110.1894$.

Von der Heyden, Das Rechenlineal (règle à biseau modifiée par Mannheim). Zeitschr. f. mathem. u. naturw. Unterricht, herausgegeben von J. C. V. Hoffmann. Jahrg. 27, 568. Leipzig 1896. F. W. Hill, The Hatchet Planimeter. Philosophical Magazine (5) 38. 265. 1894.

Fr. Hohmann, Das Linear-Roll-Planimeter (Hohmann-Coradi). Erlangen.

Hü ser, Das Mönkemöllersche Planimeter. Zeitschr. f. Vermessungsw. 25, 443. 1896.

$\mathrm{K}$. Hunaeus, Die geometrischen Instrumente der gesamten praktischen Geometrie. 2. Ausg. Leipzig 1882. [Planimeter von Ho- 
grewe. Oldendorp, Oldenburg, Wagner. Schmidt, Horsky, Westfeld, Hermann, Deeker, Keller, Fichtbauer, Sang, Amsler, Wetli, Hansen erwäbnt.]

L. Jacob, Le calcul mécanique. Encyclopédie scientifique. (Direction Dr. Toulouse) Bibliothèque de mathématiques appliquées (directeur M. d'Ocagne). Paris 1911.

L. Jermann, Die Gunterskala. Hamburg 1888.

Jordan, Polarplanimeter und Rechenschieber ron Dennert und Pape.

Zeitschr. f. Vermessungsw. 6, 290. 1877.

-, Referate in der Zeitschrift fïr Vermessungswesen.

Kelling, Diridieren auf Additionsmaschinen. Zeitschr. f. Termessungsw. 31, 171. 1902.

F. Kloht, Kombiniertes Planimeter. Zeitschr. f. Instrumentenk. 5, 41. 1885 .

-, Über ein nenes Planimeter. Zeitschr. f. Vermessungsw. 12, 97. 1883.

Koller, Proportionalrechenschieber ron $\mathrm{Ch}$. Hamann in FrieclenauBerlin. Zeitschr. f. Vermessungsw. 28, 660. 1899.

A. Korselt, Über den Trakteriographen von Kleritj und das Stangenplanimeter. Zeitschr. f. Math. und Flussik. 43, 312. 1898.

Kummer, Genauigkeit der Flächeninbaltsberechnungen mittels der Klohtschen Hyperbeltafel. Zeitschr. f. Vermessungsw. 32, 686 . 1903.

F. W. Lanchester, The radial cursor, a new addition to the sliderule. Philosophical Magazine (5) 41,52.

Lang, Das Kompensationspolarplanimeter ron G. Coradi in Zürich. Zeitschr. f. Vermessungsw. 23, 353. 1894.

F. Lorber, Über das Präzisionspolarplanimeter Hohmann-Coradi. Zeitschr. f. Instrumentenk. 2, 327. 1882. Sitzungsber. der k. Akad. d. Wissensch. math.-naturw. Kl. Wien, $188^{\circ}$.

-, Über die Genauigkeit der Planimeter. Österreich. Zeitschr, f. Berg- u. Hüttenwesen. 31. 1883

-, Ưber das freischwebende Präzisionsplanimeter ron Sohmann-Coradi. Zeitschr. f. Vermessungsw. 13, 1. 1884.

-, Über Coradis Kugelplanimeter. Zeitsch. f Vermessungsw. 17, 161. 1888.

-, Ein Beitrag zur Justierung des Polarplanimeters. Zeitschr. f. Vermessungsw, 12, 45\%. 1883.

H. Lossier, L'intégraphe Abdank-Abakanowicz. Zürich 1903 [Coradi]. Zeitschr. f. Instrumentenk. 24, 213. 1904.

Luedecke, Die Rechenapparate ron Julius Jilleter in Zürich. Zeitschr. f. Vermessungsw. 20, 34t. 1891.

K. Lüdemann, Über logarithmische Rechenschieber. \%eitschr. f. Vermessungsw. 36, 211. 1907. [Enthält eine historische l"bersicht.]

-, Über die Genauigkeit von Fläehenberechnungen mit der Quadratmillimeterglastafel. Zeitsehr. f. Vermessungsw. 36, 373. 1907.

J. E. Mayer, Das Rechnen in der Technik und seine Hilismittel. Leipzig 1908. [Sammlung Göschen.]

R. Mehmke, Stetige Rechenapparate und -maschinen. Enzyklopidie der mathematischen Wissenschaften I. Heft $\boldsymbol{i}$. 
A. A. Michelson and S. W. Stratton. A new harmonic analyser. The American Journal of science (4) 5 ( $\mathbf{4 5}$ der ganzen Reihe). Nr. 25, 1. New Haven 1898.

C. H. Nüller, Der logarithmische Rechenstab. Jahresbericht des Kaiser Friedrichs Gymnasium. Frankfurt a/M. 1899. Programm Nr. 411.

F. Müller, Die Planimeter von Gangloff und Schlesinger. Zeitschr. f. Vermessungsw. 8, 150. 1879.

R. Nelting, Der Nautisch Astronomische und Universal-Rechenstab. Hamburg 1909.

A. Nestler, Der logarithmische Rechenschieber und sein Gebrauch. Lahr i/B. 1908.

Neuendorff, Über ein neues Koordinatenplanimeter ron Ch. Hamann in Friedenau-Berlin. Zeitschr. f. Vermessungsw. 27, 553. 1898.

E. Orlich, Aufnahme und Analyse von Wechselstrom-Kurven. Elektrotechnik in Einzeldarstellungen, herausgeg. จ. Dr. Benischke, VII. Braunschweig 1906, Vieweg u. S.

-, Über Aufnahme von Wechselstromkurven durch Oszillographen und ihre Analyse. Archiv der Mathem. u. Physik (3) 12, 237. 1907.

-, Referate in der Zeitschrift für Instrumentenkunde.

A. Ott, Das Wesen und der Gebrauch des Ott'schen Kompensationsplanimeters. Kempten (Bayern).

G. Oldenberger, Einige einfache mechanische Rechenapparate. Zeitschr. f. Instrumentenk. 5, 163. 1885.

E. Pascal, Repertorium der höheren Mathematik, dentsch von A. Schepp. Leipzig 1900, B. G. Teubner. [Teil I. Kap. XXIII. Analytische Instrumente und Apparate.]

-, Lintegratore meccanico per le equazioni lineari differenziali di $1^{0}$ ordine e per altre equazioni differenziali. Atti della R. Accademia dei Lincei. 18, 304. Roma 1909. Giornale de Mathematiche di Battaglini (3) 1 (Vol. 48) 1910.

-, Sopra una semplice ma notevole variante nella costruzione dell' integrafo di Abdank-Abakanowicz. Rend. della $\mathrm{R}$. Accademia delle Scienze Fisiche e Mathematiche di Napoli, Fasc. $5^{0}, 6^{0} .1911$.

-, Sopra alcuni classi di integrafi per equazioni differenziali. Rend. della R. Accademia delle Science Fisiche e Mathematiche di Napoli. Fasc. $7^{0}, 8^{0}, 9^{0}$. 1911.

-, Di un nuovo integrafo per quadrature ed equazioni differenziali (integrafo polare). Rend. della R. Accademia delle Science Fisiche e Mathematiche di Napoli. Fasc. $10^{\circ}, 11^{\circ}, 12^{\circ} .1911$.

-, L' uso e le applicazioni dell' integratore meccanico per le equazioni differenziali. Giornale de Hathematiche di Battaglini. (3) 2 (Vol. 49). 1911.

Perry, Remarks on Prof. Henrici's Paper, in which he describes a simple machine which may be used to develop any arbitrary function in series of functions of any normal forms. Philos. Magazine (5) :38, 125.1894.

R. Proell, Ein Rechenschieber in Tafelform. Deutsche Mechanikerzeitung 22, 213. 1901. 
Puller, Rechenscheibe mit Glasläufer und Lupe. Zeitschr. f. Vermessungsw. 30, 296. 1901. Zeitschr. f. Arch. und Ingenieure. Heft 2. 1900 .

Rechenmaschine „Brunsviga“" Braunschweig (Grimme, Natalis \& Co.) 1910 .

- „GauB" Verlag von ReiB, Liebenwerda. 1905.

F. H. Reitz, Korrektur des Amslerschen Planimeters u. Konstruktion zweier neuer Varietäten desselben. Zeitschr. f. Vermessungsw. 7, 249. 1878.

-, Theorie des Amslerschen Planimeters. Hamburg 1868.

-, Rollplanimeter Hohmann-Coradi. Zeitschr. f. Vermessungsw. 13, 479. 1884.

Runge, Das Stangenplanimeter. Zeitschr. f. Vermessungsw. 24, 321. 1895.

Ad. Schmidt, Ein Planimeter zur Bestimmung der mittleren Ordinaten beliebiger Abschnitte von registrierten Kurven. Zeitschr. f. Instrumentenk. 25, 261. 1905.

Schleiermacher, Das Stangenplanimeter von Prytz. Zeitschr. f. Vermessungsw. 27, 408. 1898.

J. Schnöckel, Graphische Integrationen. Zeitschr. f. Vermessungsw. $32,129.1903$.

-, Beiträge zur Flächenberechnung mit der Hyperbelglastafel. Zeitgchr. f. Vermessungsw. 32, 369. 1903.

M. Schnyder, Das Linearplanimeter Weber-Kern. Schweiz. Bauzeitung. 51, 124.1908.

A. Schreiber, Zur Theorie des Stangenplanimeters. Zeitschr. f. Vermessungsw. 37, 689. 1908.

-, Das Präzisionsstangenplanimeter. Zeitschr. f. Vermessungsw. 38, 401. 1909.

A. Schülke, Vierstellige Logarithmentafeln. 7. verb. Aufl. Leipzig u. Berlin 1909, B. G. Teubner.

J. W. G. Schulz, Die Hamannsche Rechenmaschine „GauB“. Zeitschr. f. Instrumentenk. 26, 50. 1906.

E. Selling, Eine neue Rechenmaschine. Berlin 1887.

Semmler und Schulz, Die Rechenmaschine "Gaub" und ihr Gebrauch. Zeitschr. f. Vermessungsw. 35, 10. 1906.

Serret, Lehrbuch der Differential- und Integralrechnung. 3. Aufl. v. G. Scheffers. Leipzig 1907, B. G. Teubner. Bd. 2.

A. Sharp, Harmonic analyser. Philosophical Magazine (5) 38, 121. 1894.

H. S. H.Shaw, The theory of continuous calculating machines. Philos. Transactions of the R. Society of London. 176. Part II, 367. London 1886.

-, Mechanical Integrators. The van Nostrand Science Series Nr. 83. New York 1886.

H. Sossna, Ergebnisse einer Zuverlässigkeitsuntersuchung mit der Rechenmaschine Brunsviga. Zeitschr. f. Vermessungsw. 30, 636, 1901 (vgl. 28, 665, 1899).

-, Ergebnisse einer Zuverlässigkeitsuntersuchung eines aus der Fabrik 
von A. Nestler hervorgegangenen Rechenschiebers. Zeitschr. f. Vermessungsw. 34, 657. 1905 .

H. Sossna, Auflösung der Aufgabe des Einkettens mittelst Maschine und numerisch-trigonometrischer Tafel. Die neue Multiplikationsmaschine von Otto Steiger und Hans W. Egli in Zürich. Zeitschr. f. Vermessungsw. 28, 665, 1899.

Stampfer, Über das neue Planimeter des Ingenieurs Caspar Wetli. in Zürich. Sitzungsber. der math. naturw. Kl. der k. Akademie der Wissensch. zn Wien. 4, 134. Wien 1850.

Ch. A. Vogler, Allgemeines Prinzip des Rechenschiebers. Zeitschr. f. Vermessungsw. 10, 257. 1881.

F. A. Willers, Zum Integrator von E. Pascal. Zeitschr. f. Mathem. und Physik. 59, 36-44. 1910.

Wilski, Rollenschiefe und Scharnierschiefe beim Amslerschen Polarplanimeter. Zeitschr. f. Vermessungsw. 22, 609. 1892.

R. Wolf, Handbuch der Mathematik, Physik, Geodäsie u. Astronomie. Zürich 1870. [Bd. I. S. 192. Planimeter Oppikofer usw.].

G. U. Yule, On a simple form of harmonic analyser. Philosophical Magazine. 39, 367, 1895.

Vgl. auBerdem verschiedene Literaturnachweise im Text und in den Anmorkungen. 


\section{Register.}

(Die Ziffern bedeuten die Seitenzahlen.)

Abdank - Abakanowicz $157,162,163,169$ Abelsche Gleichung $172,176,177$

Abwicklung 49

Adder 28

Additioneur 28

Additionsmaschine 29,53

Adiabatische Kurre 14

Ajello 172

Äquidistanzplanimeter 67,70

Amsler 79, 82, 90,96, 100,118

Analysator $133,134,138$, $140,143,148$.

Archimedes 29

Arithmometer 29

Arithmotyp-Trinks 33

Auslöschvorrichtung 29 , 48

Austria 29

Babbage 44

Barometrische Höhen 17

Barthelmes 25

Bauschinger 45

Beilschneidenplanimeter 122

Bernard 21

Bernoullische Gleichung 172

Berolina 33

Beschleunigung 62

Beyerlen 22

Billeter 21

Biquadratische Gleichung 20

Blater 40

Bollée 24, 29, 39

Boucher 22

Boys 15, 157

Breithaupt 89

Brigg 1
Brunsriga 33

Brunsvigula 3:3

Bryand 116

Büttner 34

Buniakowsky 100

Bunzel 29

Burkhardt 2i, 29

Burrough 25, 28

Byrgi 2

Calculex 22

Cayley 157

Cercle à calcul 을

Clouth 2.

Compteur 24

Le Conte 137

Contostrie 24

Coradi 64, 91, 103, 104, $105,121,131,140,1 \$ 1$,

$15 \%$

Coriolis 157

Decker 100

Delton 29

Differentialkurve $155 \mathrm{ff}$.

Differentialquotient 59

Differentialwagen $157 \mathrm{ff}$.

Differenzenmaschine $\mathbf{2 9}$, 44

Direktrix $155 \mathrm{ff}$.

Direktrixschiene $158 \mathrm{ff}$.

Division 10, 12, 22,32,54

Durand 118

Dyck 133

Edmonson 34

Egli 39

Einmaleinskörper $41 \mathrm{ff}$

Encke 169

Erleichterungstafel 40

Ernst 81

Exponentialgleichung 15

Exzentrizität 85
Euler 169

Everett 21

Faber, A. W, 5

Favaro 17

Fernel 63

Fieguth 127

Finsterwalder 133

Fleischhauer 65

Frank 16, 68

Fourier 131, 132

Eranzösisches Kataster 17

Fiirle 15, 19

Fuller 21

Gangloff 69

G a B B, C. F., 169

GauB (Maschine) 23, 34

Genauigkeit 15, 16, 63, $66,82,109,151$

Generalkommission

Kassel 21

Geschwindigkeit 59, 61

Goering 4

Goldman 24

Gonella 51, 78, 80, 81

Goodman 127

Grabowshi 143

Graret 4

Grundkreis 101

Grob 145

$G$ ünther 67

Gunter 3

Hahn 29, 34

Halden 22

Hamann $30,34,45.53$, $54,56,67,82$

H a m m e r $3,63,65,69$, 118

Hannyngton $\mathbf{1 7}, 21$

Hansen $50,81,111$

Harfenplanimeter 67

Hart 2.2 
Hartmann 37

Hatchet-Planimeter 122, 127

Henrici 140, 141

Hermann 22, 80, 100

Hoecken 56

Hofmann 69

Hohmann 91, 103, 104, 105

Hyperbolische Funktionen 56

Hyperbelglastafel 66

Hyperbelplanimeter 78 , 88

\section{Ideal 29}

Integralkurve $130,155 \mathrm{ff}$. Integrallogarithmus 169 Integralwagen $157 \mathrm{ff}$.

Integratore meccanico 161

$\mathbf{J}$ acob $64,172,176$

Jordan 21

Kartometer 65

Kegelräder 26, 27, 42

Kelvin 138, 172

Kern 127

Kewitsch 2

Kloht 111

Kloth 66

Klott 21

Knudsen 122

Koblmorgen $\mathbf{1 1 4}$

Kombiniertes Planimeter 111

Kompensationsplanimeter 130

Kompensationspolarplanimeter 110

Kontrollineal 84, 99

Koordinatenplanimeter $77,82,99$

KrauB 63

Kronecker 169

Kubikwurzel 13

Kubische Gleichung 20

Kubus 13

Küntzel 142

Küttner 34
Kugelpolarplanimeter 105

Kugelrollplanimeter 96

Kurvenzylinder 116, 139

Längenteilung 8, 16

Läufer $4,14,69$

Lambert 4

Lanchester 14

Leibniz 25, 29, 33

Lenoir 4

Linearplanimeter 87,89 , 90, 91, 99, 109

Lippincott 127

Logarithmen 1, 2, 8, 10, 45,56

Logarithmischer Rechenschieber 1

Logistik 2

Lii d emann 22,66

Mannheim 4, 21

Martiny 14

Maxwell 52, 100

Mayer 25,64

II ehmke 22, 33

Mercedes-Euclid 30

Merkur 33

Meyer 22

Michelson 148

Miller v. Hauenfels 89,100

Millionär 39

Mönkemöller 67,70

Momentenplanimeter 118,121

Nonopol 33

M üller, C. H., 2

Müll er, J. H., 29, 34

Multiplikation 10,12,21, 40,54

Multiplikationsmaschine $29,37,39,44$

Multiplikationsmechanismus 40

Napier 2

Nautischer Rechenstab 17

Nelting 17
Neper s. Napier 40

Nestler 4, 16

Netto 169

Nürnberger Scheere 37

d'Ocagne 156

Odhner 23, 33

Oldendorp 67

Omnimeter 22

Oppik ofer 81

Optisches Planimeter 129

Ordinatenkreis 108

Ordnungsziffer 9

Ott 65

Oughtred 3

Panintegrimeter 114

Pascal, B., 24, 25

Pascal, E., 161, 169, $170,171,172$

Patridge 3

Peerless 29

Peters 45

Petrovitch 172

Planimeter für Kugel 110

Planimeterhaarzirkel 67

Planimeterschieber 68

Planimeterzirkel 67

Platzbecker 63

Polarintegraph 171

Polarplanimeter 79,99 , 100,109

Poncelet 51

Potenz 14

Präzisionsplanimeter $103,104,105,109$

Pregél 129

Proell 21

Progrebstab 2

Proportionalhebel 30

Proportionalrechenschieber 53

Prüfung dez Rechenschiebers 15

Proportionalrechnung 12

Proportior 2:

Prytz 122, 128, 131, 172

Puller 22 
Quadratbildung 12, 17 Quadratische Gleichung 11

Quadratmillimeterglastafel 66

Quadratwurzel 13,17,55

Radial cursor 14

Radialplanimeter 118

Rechenknecht 22

Rechenkreis $\mathbf{2 2}$

Rechenräder 22

Rechenscheiben 22

Rechenschiever 22,23 , 133

Rechenstäbe 1, 40

Rechentafeln 21

Rechenwalzen 21

Registrierwerk 40, 63

Registrierzylinder 139

Reitz 93

Rektifizierrädchen 63

Reuleaux 25

Riccatische Gleichung 172,173

Riffelnng 76

Roether 22

Rollen - Kugel - Mechanismus $51,54,55,56$. $61,97,105,110,139$, 142,143

Rolleu - Scheiben - Mechanismus $50,59,78$, 81. $91,103,104,112$. $117,118,119,139,146$ Rollenschiefe 85

Rollplanimeter 88, 91

Roth 28

Runge 123

v. Sanden, H., 128

Sand or 64

Sang 87

Saxonia $\mathbf{2 9}$

Scharnierschiefe 86, 111

Scherer 21

Schieber s. Zunge 3

$\mathrm{Schlagintweit} 64$

Schlesinger 69
Schmidt, A., 116, 133

Schnöckel 122, 129

Schreiber 133

Schütz 45

Selling $3 \pi, 39$

Semmler 67

Sexton 22

Sharp 141, 143

$\mathrm{H}-\mathrm{S}$ haw $54,59,62,121$

Sinusskala 6,18

Smollin 78

Soho rules 3

Soldner 169

Sommerfeld, A., 145

Sonne 22

Sresnewsky 17

Stadler $78,80,88$

Staffelwalze 25, 26

Stangenplanimeter 122, 129

Stanley 21

Starke 81

Statisches Moment 119, $120,122,161$

Steiger 39

Steinbauser 2 ?

Stellbebel 33

Strachey 133

Stratton 148

Stufenscheibe 35

Tachymetrische Skalen 17

Tangens-Teilung $6,8,18$

Taschenrechenschieber 5

Taschenschnellrechner $\geq 2$

T'avernier 4

Terada 152

Tesdorpf 65

Thacher 21

Thomas $27,29,41$

Thomson s. Kelvin 138 , 140,157

Tim 29

Torrès 172

Trügheitsmoment 119, $121,122,126,162$
Trigonometrische Funktionen 56

Trinomische Gleichung 20

Triumphator 33

Ċbertragungsmechanismus 40

Übertragungsvorrichtung 24 s. Zehnerübertragung

Umfahrungsfehler 109

Unitas 29

Verwandlungsplanimeter 69

Vinay 4

Volkmann 145

Waelzen 52

Wabrscheinlichkeit 162

de Wal 68

Warnungszeichen 29,31

Watt 3

Webb 2s

Weber 22, 127

Weltausstellung Brüssel 21

Wetli $81,86,111$

Wiberg 45

Wichmann 4, 22

Wiechert 145

Wingate 3

Wittmann 63

Wolf 64

Wurzelziehung 14,55

Yule 134, 135

Zählwerk 23, 31, 33, 36, 48,49

Zehnerübertragung 24 , $27,38,41,48$

Zenitdistanz 18

Zinseszinsrechnung 20

Zirkeladdition 67

Zmurko 15 i

Zunge $3,17,69$ 
Druck von B. G. Teubner in Leipzig 


\section{Verlag von B. G. Teubner in Leipzig und Berlin}

\section{Wirkungsweise und Gebrauch des Mikroskops und}

seiner Hilfsapparate. Ton Prof. Dr. W. Scheffer. Mitit s 9 Abbildangen nnd

geb. «. 3.-

3 Blendenblättern. 1911. Geh. \&h. 2.40, in Leinwand

Auf fast allen Gebieten der Naturwissenschaften and der Technik ist das Mlikroskop ein wichtiges - häafig ein unentbehrliches - Hilfsmittel geworden. Zum Verständnis und zur richtigen Anwezdung dos Mikroskops sind einige physikalische Kenntnisse notig. Im vorliegenden Bnche werden die notwendigen physikalischen Grundlagen möglichst allgemeinverständlich und einfach vorgetragen, so das jeder Gebildete der Darstellung leich folgen kann. Durch eine größere Anzahl von Figuren, sowohl Zeichnungen als auch Mikrophotogrammen wird das Verständnis dem Leser erlcichtert. Die Beschreibung einer Reihe einfacher Experimente gibt dem Leser Gelegenheit, die Vorgänge in praxi wahrzanehmen. Die Erscheinungen in der hinteren Brennebene, deren Kenntnis für das Verständnis des Mikroskops ganz besonders wichtig ist, werden durch Experimente mit den dem Bnche beigegebenen Blendenblättern auf einfache Weise klargemacht.

\section{Maschinen und Apparate der Starkstromtechnik, ihre Wirkungsweise und Konstruktion.} zam Selbststudium and für den in der Praxis stehenden Ingenieur. Von Elektroingenieur G. W. Meyer, I. Teil: Gleichstrom II. Teil: Wechselstrom. Mit 7fe Figuren. Geh. It 15.-, geb. It. 16.-

Will dem Studierenden wle dem in der Praxis stehenden Ingenieur schnelle und zuverlässige Information aber den Stand des Baues modcrner elektrischer Maschinen und Apparate sowie uber die bei der honstruktion derselben zu beachtenden fresichtspunkte bieten. Soweit zum Verständnis der betreffenden Haschinen und Apparate erforderlich, wurde anch auf die Theorie derselben kurz eingegangen. Za dem gleichen $Z$ wecke dient eine groBe Zahl graphischer Reproduktionen.

Populäre Astrophysik. physikalischen Observatorium bei Potsdam. Mit 30 Tafeln und 210 Figuren. gr. s. $190 s$. In Leinwand geb. Wh 12 .-

"... Sein Hauptrorzng besteht darin, das es den Leser zunächst auf das sorgfaltigstemit den astrophysikalischen liethoden und Instrumenten vertraut macht; fast die Hälfte des Buches ist diesem Zweck gewidmet. Dadurch ist es aber nicht etwa za einem Handbuch für den Fachmann geworden, nein, es ist eine gemein-verständliche Darstellang im besten Sinne des Wortes fur den groBen Kreis der Gebildeten Mathematische Betrachtnngen, die nun einmal nicht au entbchren sind, werden vicht ängstlich vermieden; sie gehen aber nirgends aber den standpankt eines Gymnasialprimaners hibaus. Überall schöpft der als hervorragender Foracher bekannte Verfasser ats dem vollen."

(Monatsschrift für höhere Schulen.)

Geodäsie. Fine Anleitung zu geodätischen Messungen fur Anfänger mit GrundProfessar an der Techuischen Hochschule zu Darmstadt Wit 216 Figtren. 1910.1 . Leinwand geb. „H. 12 -

Das Buch soll fur die meisten technischen $Z$ wecke ansreichen und zwischen den unfangreichen Handbachem und den kleinen Leitfaden stehen. Deshalb wurden die (irundzuge der Wassermengen- und Wasserkraftmessung in Wasserläufen aufgenommen, und auch dle Beschreibang einiger Methoden zur direkten (astronomischen) Bestimmung der geographischen Koordinaten von Punkten der Frdoberfläche sowie der Azimute terrestrischer Rlchtangen mit Hilfe des Theodolits wird manchem erwhuscht sein. Der Beschreihut sowie der Berichtignag der MeBingtrumente ist verhaltnismasif viel Raum zagewiesen, weil erfalırungəgemä $\beta$ das Fintstelen unbrachbarer Mlessungen am meisten durch ungenlagendes Vertrautsein mit dem MeBgeräte begünstigt wird. Iie Messnngsand Berechnnogsarteu sind durch viele Zahlenbelspiele erläutert, und ancl an kiguren zur Unterstutzung des Textes ist nicht gespart.

"... Die Darlegungen sind samtlich klar und auch dem Anfanger wohl leicht verståndicli; sie werden durch zahlreiche Figuren erläutert und durften besonders für dit? Studierenden der FcldmeBknnst wertvoll sein. llier liagt vlelnehr die llauptbedeatung in der Art der Darstellung eines schon häug behandelten Ftoffes, und diese 1)arstellung verdient in diesem Falle das höchste Iob." (Literarisches Zentralblatt für Deutschland.) 


\section{Verlag von B. G. Teubner in Leipzig und Berlin}

\section{Lehrbuch der Physik Zum Gebrauch beim Unterricht, bei akademische}

Uorlesungen und znm Selbststudium von E. Grimseh Direktor der Oberrealschule auf der Uhlenhorst in Hamburg. Mit 129; Textfiguren 2 farbigen Tafeln und einem Anhange, enthaltend Tabellon physikalischer Konstantex und Zahlentabellen. 2. Auflage. 1912. Geh. Wh 15., in Leinwand geb. Mh 16.-

"... In erster Linie sei hier das Werk von, Grimsehl' erwähnt, nach Anffassung nnc Durchfillırung wohl das größte und schönste Lehrbuch der Experimentalphysik, welche an höheren Schulen benutzt werden soll. Wir können stolz darauf sein, daB ein Fach. kollege hier ein Werk vorlegt, welches als Handbuch der Physik den angehenden Studenter dieses Faches ebensowohl wio den Medizinern, Chemikern und Pharmazeuten meine Uberzeugung nach bessere Dienste leisten kann und wird als verschiedene bekannte Lehr. bïcher, dic wir seinerzeit auf der Universität in Händen hatten...."

¿Korrespondenz-Blatt für den akademisch gebildeten Lehrerstand.

"Was es für diese Zwecke besonders geeignet macht, ist die horvorragend klare unc anregende Art der Darstellung. Jeder Abschnitt geht von einfachen Beobachtungen und leicht anzustellenden Versuchen aus. Uber tausend Abbildungen, zumeist sind es rech charakteristi che schematische Zeichnungen, nnterstatzen den Text in wirksamer Weise

Mit einem Worte, das Buch verdient in wissensehaftlicher, methodiseher und didaktisches Hinsicht volle Anerkennung."

\section{Taschenbuch für Mathematiker und Physiker. $\underset{\substack{\text { Nnter Mit } \\ \text { wirkung }}}{\text { Mit }}$} zahlreicher Fachgenossen herausgegeben von Felix Auerbach und Rudolf Rothe. II. Jyhr gang 1910/11. Mit einem IBildnis Hermanu Minkowskis. 1911. In Leinwand geb. M. 7.-

Das Taschenbuch enthält Augaben über Personalien, Literatur, Praktigches usw., haupt. sächlich aber ein Gerippe des Tatsachenmaterials der mathematischen und physikalischev Wisgenschaften, zu denen noch Astronomie, Geodäsie, Flektrotechnik nnd physikalisclie Chemie als Annexe hinzugefügt wurden, um allseitigen Bedürfnissen entgegenzukommen. Die bei der er sten Herausgabe des Werkes geäuBerte Annahme, es kommo einem dringeud empfundenen Tedürfnisse entgegen, hat bei Publikum und Kritik eine volle Bestätigung gefunden, und das Buch hat sich als praktisches Hilfsmittel bei Mathematikern, Physikern und Angehörigen verwandter Gebiete rasch verbreitet. Bei der Herausgabe des zweiten Jahrgangs hat sich die Notwendigkeit herausgestellt, das in die Redaktion ein zweiter, mathematischer Herausgeber eiutrat, und daB dieser eine völlige Nenbearbeitung des mathematischon Teiles in die Wege leiten muste. Anch der phvsikalische, astronomische und chemische Teil enthält, unter Fortlassung einzelner Absclinitte des ersten Jahrganges, sehr viel des Neuen; daß im übrigen Mängel des fräheren Textes beseitigt und berechtigte Wüsche aus dem Leserkreise beachtet wurden, versteht sich von selbst. AuBerdem hat sich das Taschenbuch diesmal der Mitwirkung einer gröBeren Anzahl von Fachgenossen zu erfreuen, die os in dankenswerter Weise ibernahmen, gewisse besondere aktuelle Kapitel in Sonderdarstellungen rorzuführen. [Jahrgang III erscheint im November 1912.]

Die Reichhaltigkeit und Vielseitigkeit, die sich schon beim Durchblättern zeigen, und die Gediegenheit des Inhalts, die sich dem eiugehenderen Studium erschlieBt, machen im Verein wit der łbersichtlichen Stoffanordnung das Taschenbuch zn einem Orientierungs mittel von großer Verwendbarkeit und Zuverlässigkeit; jedem Freunde der exakten Wissenschaften kann daher die Anschaffung des vorzüglichen Buches angelegentlich cmpfohlen werden."

\section{Kröhnkes Taschenbuch zum Abstecken von Kurven

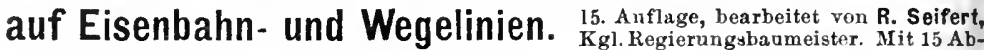

\section{bildungen. 16. 1911. In Jeinwand geb. $\mathscr{M}, 2$.}

Das seit 1851 in fünfzehn Auflagen erschieneno ,Taschenbuch " soll die beim Abstecken von Bðgen in Eisenbalın- und Wegelinien erforderlichen Rechnnngen nach Mögliehkeit erleichtern und vor Felılern sichern. Als Hauptverfahren ist die Absteckung von Bogenpunkten gleiclien Abstands von der Tangente aus mit rechtwinkligen Koordinaten zugrande gelegt. Die Einleitung enthält die hierfür nötigen mathematisehen Entwicklungen und Hinweise auf die zur Aushilfe dienenden anderen Verfahre: Korbbögen und Übergangsbögen von Fisenbahnlinien gind in die Betrachtung einbezogen worden. Fermer ist eine Anleitung zur Winkelmessung in dem für Bogenabsteckung erforder]ichen Umfang und zur Prüfung und Beriehtigung des Theodoliten gegeben.

Die eigentlichen Zahlentafeln sind in 3 Abteilnngen gegliedert; Tafel I enthält alle Werte zur Berechnung der 'Tangentenlängen und der Kontrollen der Absteckung, Tafel II a]le Ordinaten und Abszissen zur Bogenabstecknng, Tafel III das Winkelmaß für bestimmte Bogenlängen. Der Umfang der 'Tafeln beztiglich der Abstufung der Halbmesser von 20 bis $10000 \mathrm{~m}$, der Winkel von $10 \mathrm{zu} 10$ Minuten und der Länge der Ordinaten bis $\mathrm{zu} 100 \mathrm{~m}$ dürfte allen Anforderungen der Bequemliehkeit der Rechnung und der Genauigkeit gent' gen. 


\section{H. Wiener und P. Treutleins Sammlung mathematischer} Modelle für Hochschulen, höhere Lehranstalten und technische Fachschulen. Die Modelle sind fiur den geometrischen Unterformen and geometrisehe Beziehangen durch einfache und ubersichtliche Darstellng anschanlich machen. Das Preisverzeichnis mit Fignren und Tafeln nach photographisehen Aufnahmen der Modelle ist dureh den Jerlag an. berechnet $z$ u beziehen.

Die elementare Mechanik. Fin Lehrbnch: Enthaltend: Eine Begrindung Systeme starrer Körper; die synthetischen and die Elemente der analytisehen Methoden sowie eine Einführung in die Prinzipien der Mechanik deformierbarer Systeme. Yon Georg Hamel, Professor an der Dentzehen Technischen Hochschnle zu Brün. Mit 265 Figuren, 1912. Geh. wh 16.-, in Leinwand gel, wh 18.-

Das Buch enthält die Grundlagen einer allgemeinen Mechanik sowie die Stereomechanik, hingegen nicht die Mechanik der deformierbaren Körper. Behandelt es anch in seinen Beispielen hanptsächlich technische Probleme, so ist es doch keine eigentlicho technische Mechanik, sondern hat vor allem eine Darstellnng der allgemeinen Meehanik znm Ziel. Der erste Abschnitt entwickelt ansfahrlich den kinetischen Kraftbegrift, der zweite enthält die Statik, der dritte bant die allgemeine Mechanik anf die Theorie der Volumelemente auf und geht in die Systemmechanik bis za den Lagrangeschen Gleichnngen. Die beiden letzten Paragraphen zeigen, wie sieh die Begründung der Mechanik deformierbarer Körper an die allgemeinen Grundlagen anschlieBt

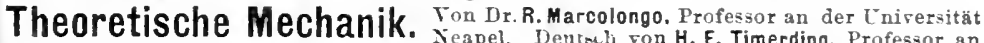
der Tieapel. Dental von H. E. Timerding, Professor an wand geb th je th 11.- I. Band: Kine 2 Bande. gr. 8. Geh. je w 10 - in LeinII. Band: Dynamik und Mechanikderdeformierbaren Körper. Mit 38 Fig. 1912. Dem Werke kommt sehr zustatten, daB der Verfasser von vornherein darauf verzichtet hat, den Stoff ersehöpfend zu behandeln. Er bringt nur eine Auswahl der interessantesten Gebiete. Als besonderer Yorzag maß weiter hervorgehoben werden, daB jedem Kapitel eine reichliche Anzahl von Ubungsbeispielen mit Anflosungen heigefügt ist, an Hand derer sich der Leser vergewissern kann, oh er den Inhalt des Kapitels wirklich ganz erfaBt hat. Das Buch wird sich sicher in den Kreisen der Ingenieure viel Freunde erwerben. Fs ist so abgefaBt, daB anch der Ingenieur, soweit er far dio Theorie Interesse hat, vieles Nüzliche darin findet. Die theoretische Mechanik von Marcolongo-Timerding kann deshalb bestens empfohlen werden." (Zeitschrift des Vereins deutscher ingenieure.)

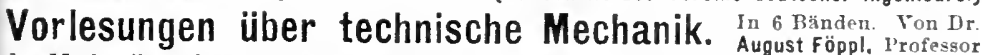
der Mechanik and Yorstand des Mechan.-Techn. Laboratorimns an der'Wechn. Hochschule in Munchen. I. Einführong in die Mechanik. 4. Anfl. Mit I04 Figuren. 1911. 4. 10,11. Band. Graphisehe Statik. 3. Anf. 1912. Mit 209 Fig. Wh-.-. I II Band. Festigkeitslehre. 4. Anfl. Mit -6 Fig. 1910. H 10.- IV. Band. Dynamik. 3. Auf. Mit i1 Fig. 1909. $\mathscr{H} 10$ - V. Band. Die wichtigsten Lehren der hoheren Elastizitätstheorie. Mjt 44 Fig 1907. H10.- FI. Band. Die wichtigsten Lehren derhoheren Dynamik. Mit30 Fig. 19j0. 16. -

„Foppl rersteht die Kanst, nit klaren und interessanten Worten, gestütat anf geistreich gewählte Beispiele, auseinarderzusetzen, was die Formel kurz aber trocken zusammenfaBt. Man gewinnt darans auf die angenehmste Art finsichten, die sich sonst hinter langen Formelentwicklungen verbergen. Die tuscinandersetzungen werden bei Föpl zwar äuBerlich länger als in der knappen Formelsprache anderer Bücher, die zum Verstiudnis notige Zeit wird aber kurzer. Jas sind Vorzäge, die fur d*n l'raktiker schwerer ins Gewicht fallen, als die bei früheren Auflagen aus mathematischen Kreisen geäuBerten Bedenken gegen die Korrektheit mancher Fintwicklnngen."

(Elektrotechnische Zeitschrift.)

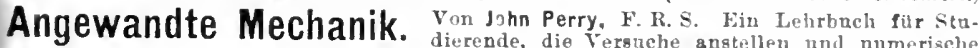
no dierende, die Ferstuche anstellen und numerische charbeiten wollen. Berchtigtc deatsche Cbersetzung ron Ingenjenr Rudolf Sohiok in Cisln. Mit 371 Figuren. 190. In Leinwand geb. $\%$ 18.-

„Ans diesem Werke spricht ein Lehrer allerersten Rages, der ausgedelınte Kenninise mit vollendeter Lehrkunst vercinigt. Fir hat aus dem groBen Wissengebiete der technisclup Mechanik viele hundert Beispiele zusammengetragen, an welelien er dic Grunlgesetze anschau. lich erläutert, and darnit ein echtes Lehrbuch geschatfen, desaen Lbergetzung sich bald zahlreiche Frennde erwerben wird. Alle larlegungen sind unmittelbar auf den praktischen tiebrauch zugeschnitten. und der mathematiche A pparatist in migljehst engen firenzen gehalten; voransgrsetxt wirl lediglieh die Kenntnis der nifder"n Analysis" (Literarisch. Zentralblatt.) 


\section{Verlag von B. G. Teubner in Leipzig und Berlin}

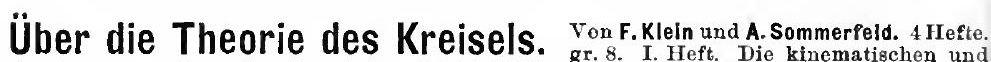
kinetischeu Grundlagen der Theorie. 1897. Geh. „1. 5.60, geb. $\mathcal{H} .6 .60$. II. Heft. Durchführung der Theorie im Falle des schweren symmetrischen Kreisels. 1898. Geh. Ah. 10.-, geb. A. 11.- III. Heft. Die störenden Einthïse. Astronomische und geophysikalische Anwendungen. 1403. Geh. Kh. 9.-, geb. Ah 10.-. IV. Heft. Dio technischen Anwendungen

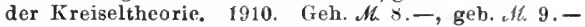

„In klarer und, soweit bei diesem Stoff uberhaupt möglich, auch anschaulicher Weise sind zuerst allgemeine Auseinandersetzungen über Stabilierung gegeben und dann die cinzelnen Anwendungen besprochen, n. a die Kreiselwirknngen bei Schnellbahnen, der Geradlaufapparat der Torpedos, der Schlicksche Schiffskreisel, der KreiselkompaB, die Stabilität des Fahrrades sowie einige Bemerkunqen über Finschienenbahnen, die Stabilierung von Flugzeugen und die Kreiselwirkungen bei Geschossen. Die wichtigeren dieser Anwendungen sind in theoretischer wie praktischer Beziehung eingehend und uberaus klar auseinandergesetzt, wobei noclı besenders anerkannt werden soll, daß überall auf die Grenzen der Gültigkeit der Formeln und auf die zu ihrer Herleitung erforderliclen Vernachlässigungen hingewiesen ist und der entstehende fehler wenigstens seinem Sinne nach angegeben wnrde, wenn seine Größe sich niclıt abschätzen ließ. Die Diskussion der Differentialgleichungen für den Schiffskreisel soll als Beispiel einer eleganten Behandlung, einer eingehend durchgearbeiteten Aufgabe sowio der Abschnitt über Ballistik als Beispiel einer vorzüglichen Klarlegung eines noch nicht gelösten Problems hervorgehoben werden... "(DinglersPolytechn.Journ.)

Technische Statik. Torlesungen uber die Theorie der Tragkonstruktionen von CCIMISCE S LIIK. A. Ostenfeld, Professor an der lechnischen Hochschule zu Kopenhagen. Detitsche Ausgabe von D. Skouge. 1901. Geb. Nl. 12.-

Das Werk trägt seinem Inhalt und seiner Behandlung des Stoffes nach - die in der Regel sowoll zeichnerłsch wie rechnerisch durchgefülırt ist - den Bedürfnissen von Hërern an technischen Hochschulen sowie von Ingenieuren der Praxis Rechnung. Dabei werden die allgemeine technische Elastizitätslehre sowie die ersten Elemente der graphischen Statik als bekannt vorausgesetzt.

Nach Vorausschickung dreier einleitender Abschnitte, in denen die Eigenschaften nnd Anwendungen der Finflublinien sowie die einfach unterstätzten vollwandigen 'I'räger und Fachwerkbalken bei ruhender und beweglicher Belastung bebandelt werden, wird im vierten A bschnitt die allgemeine Theorie der Tragkonstrnktionen einheitlich - fü statisch bestimm te nnd unbestimmte Systeme - mit Hilfe der virtuellen Verschiebnngen aufgebaut. Ein fünfter Abschnitt gibt das Wesentlichste über die verschiedenen Fachwerkformen, wobei anch die in den letzten Jahren entstandenen Formen, K-Fachwerk, halbe Diagonalen, behandelt werden.

Das ganze Werk ist durch eine wolltuende Klarheit ausgezeichnet. Mit richtigem Gefühl hat es Verfasser verstanden, allzu weitgehende, uferlose theoretische Abhandlungen zu unterlassen. Dadurch wird die dem Anfänger oft schwer verdauliche Kost schmackhaft geboten, wodurch das Verständnis wesentlich gefördert und beim Leser die Lust zur Weiterarbeit geweckt wird. Die Übersetzung ist gut gelungen, so daB man dic Abstammung des Buches aus einem auBerdeutschen Lande kaum bemerkt. Sehr praktisch ist die Anordnung der Figuren in einem besonderen dünnen Heft, welches durch einen Finschub mit dem Text fest verbunden werden kann."

(Technische Literatur.)

\section{The Dynamics of Particles and of Rigid, Elastic, and Fluid Bodies * being Lectures on Mathematical Physics. By Professor A. G. Webster. 2. Auflage. 1912. gr. 8. In Leinwand geb. At. 14.-}

Die dem Buche dies- und jenseits des Ozeans bereitete günstige Aufnahme beweist, daB es einem Bedürfois entspricht und seine Aufgabe, in gedrängter Form die dem Studenten der Physik und Mathematik nötigen hauptsächlichsten Methodeu und Frgebnisse der allgemeinen Mechanik darzubieten, wohl erfillt. Der Student lernt aus dem Buche zugleich das Prinzip der Lnergie, das Prinzip der kleinsten Wirknng und die Lagrangesehey Gleichungen in verallgemeinerten Koordinaten kennen, die ihm die Mittel zur Losung praktischer Anfgaben an die Hand geben. Der zweite Teil des Buches ist der Bewegung der starren Kërper gewidmet und dabei ein rocht erheblicher Raum der Bewegung rotierender Körper einschließlich der praktischen Anwendungen des Gyroskepes zngewiesen Der dritte Teil behandelt die Mechanik der kontinuierlichen Systeme, eingeleitet durch dif Attraktionstheorie, in welcher das Newtonsche und Logarithmische Potential den 'Studierender zur Bekanntschaft mit den partiellen Differentialgleichungen und bestimmten Integraler uberleiten. Darauf folgt die Behandlung der linearen Vektorfunktionen mit Anwendun auf Brucb und $Z$ ag. Die einfacheren Probleme der Elastizität werden in Verbindang mi der Biegung und Windung des Stabes nach St. Venant abgehandelt. In der Hydredynamil schliebt sich der Wirbel- und Wellenbewegung eine Einführung in die Theorie der Gezeiten an 


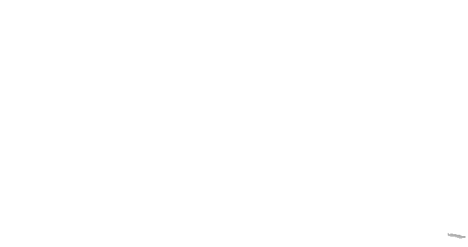





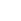





\section{PLEASE DO NOT REMOVE \\ CARDS OR SLIPS FROM THIS POCKET}

\section{UNIVERSITY OF TORONTO LIBRARY}

PQA 
